FHWA/IN/JTRP-2001/22

Final Report

EVALUATION OF SELECTED VIDEO

DETECTION SYSTEMS AT SIGNALIZED

INTERSECTIONS

Jeromy Grenard

Darcy Bullock

Andrzej P. Tarko

November 2001 
Final Report

FHWA/IN/JTRP-2001/22

\title{
EVALUATION OF SELECTED VIDEO DETECTION SYSTEMS AT SIGNALIZED INTERSECTIONS
}

\author{
Jeromy L. Grenard \\ Research Assistant \\ Darcy Bullock \\ Associate Professor of Civil Engineering \\ and \\ Andrzej P. Tarko \\ Associate Professor of Civil Engineering \\ School of Civil Engineering \\ Purdue University \\ Joint Transportation Research Program \\ Project Number: C-36-17ZZ \\ File Number: $8-4-52$ \\ Conducted in Cooperation with the \\ Indiana Department of Transportation \\ and the \\ Federal Highway Administration
}

The contents of this report reflect the views of the authors, who are responsible for the facts and the accuracy of the data presented herein. The contents do not necessarily reflect the views or policies of the Federal Highway Administration and the Indiana Department of Transportation. This report does not constitute a standard, specification, or regulation.

\author{
Purdue University \\ West Lafayette, IN 47907
}

November 2001 
TECHNICAL REPORT STANDARD TITLE PAGE

\begin{tabular}{|c|c|c|}
\hline $\begin{array}{l}\text { 1. Report No. } \\
\text { FHWA/IN/JTRP-2001/22 }\end{array}$ & 2. Government Accession No. & 3. Recipient's Catalog No. \\
\hline \multirow{2}{*}{\multicolumn{2}{|c|}{$\begin{array}{l}\text { 4. Title and Subtitle } \\
\text { EVALUATION OF SELECTED VIDEO DETECTION SYSTEMS AT SIGNALIZED INTERSECTIONS }\end{array}$}} & $\begin{array}{l}\text { 5. Report Date } \\
\text { November } 2001\end{array}$ \\
\hline & & 6. Performing Organization Code \\
\hline $\begin{array}{l}\text { 7. Author(s) } \\
\text { Jeromy Grenard, Darcy Bullock, Andrzej Tarko }\end{array}$ & & 8. Performing Organization Report No. \\
\hline \multirow{2}{*}{\multicolumn{2}{|c|}{$\begin{array}{l}\text { 9. Performing Organization Name and Address } \\
\text { Joint Transportation Research Program } \\
\text { Civil Engineering Building } \\
\text { Purdue University } \\
\text { West Lafayette, Indiana } 47907-1284\end{array}$}} & 10. Work Unit No. \\
\hline & & $\begin{array}{l}\text { 11. Contract or Grant No. } \\
\text { SPR-2391 }\end{array}$ \\
\hline \multirow{3}{*}{$\begin{array}{l}\text { Indiana Department of Transportation } \\
\text { State Office Building } \\
100 \text { North Senate Avenue } \\
\text { Indianapolis, IN } 46204\end{array}$} & & $\begin{array}{l}\text { 13. Type of Report and Period Covered } \\
\text { Final Report }\end{array}$ \\
\hline & & \\
\hline & & 14. Sponsoring Agency Code \\
\hline
\end{tabular}

\section{Supplementary Notes}

Conducted in cooperation with the U.S. Department of Transportation, Federal Highway Administration, NCP 4D4e0212.

\section{Abstract}

Video detection technologies have been emerging in recent years as a viable alternative to inductive loop detectors to actuate intersection signal controllers. There is a need for quantitative evaluation procedures for documenting the performance of video detection technologies. This quantitative data is needed to qualify systems for procurement and provide benchmarks for training traffic signal technicians that deploy and maintain these systems. This research makes use of two new evaluation procedures for video detection, with several measures of performance. The first of these evaluation procedures involves the comparison of the occupancy times of inductive loop detectors and video detectors to find the amount of discrepancy between the two. The second evaluation method involves calibrating a statistical model in order to determine which weather and traffic characteristics have the greatest effects on the operation of video detectors. This report describes the test facilities used, the evaluation methods used, and documents results of this research. Problems documented in this report were independently corroborated by INDOT personnel by conducting spot inspections at over a dozen intersections with deployed video detection systems.

7. Key Words
video detection evaluation, likelihood analysis, video detector error, inductive loop
detector error, sensitivity analysis
detector error, sensitivity analysis

\section{Distribution Statement}

No restrictions. This document is available to the public through the National Technical Information Service, Virginia, 22161

\author{
19. Security Classif. (of this report) \\ Unclassified
}

\author{
20. Security Classif. (of this page) \\ Unclassified
}

\begin{tabular}{c|c} 
21. No. of Pages \\
199
\end{tabular}

22. Price 


\section{TECHNICAL Summary}

INDOT Research

Technology Transfer and Project Implementation Information

TRB Subject Code: 54-5 Traffic Control Devices

Publication No.: FHWA/IN/JTRP-2001/22, SPR-2391

November 2001

Final Report

\section{EVALUATION OF SELECTED VIDEO DETECTION SYSTEMS AT SIGNALIZED INTERSECTIONS}

\section{Introduction}

Vehicle detectors are important in regulating traffic signals based upon demand, and not on a fixed time. Since the beginning of the use of actuated signals in the late 1970's, inductive loops have been the primary detection device used by the Indiana Department of Transportation. The use of video detectors for vehicle detection has recently become more attractive due to advancements in the technology and a drastic reduction in costs. Despite the attractiveness of the technology and the low costs, there is a lack of evaluation methods and procedures for different video detection systems. There is also a need of specialized training for those personnel that will be involved with the deployment and maintenance of video detection systems in the field.

This research project developed an evaluation system at the intersection of Northwestern and Stadium Avenue in West Lafayette that had cameras mounted on all four approaches as well as traditional stop bar loop detectors. The video cameras were connected to two different video detection systems so that three independent systems could be evaluated simultaneously - video detection system 1 , video detection system 2 , and loop detectors. In addition, the phase information was recorded. All this information was overlaid on video that was recorded during tests.

\section{Findings}

Based upon this work, nighttime detection appears to be of the most concern. Two types of problems were observed:

- The effective length of the detection zone increased from an average of 23.7 $\mathrm{ft}$ during the day to an average of $67.7 \mathrm{ft}$ at night. This has a negligible impact on safety. However, signals operate less efficiently at night because they do not gap out when they should.
- Lost detection when vehicles pull past the stop bar. Loop detectors typically do not lose a call in these situations, because the back of the vehicle is still in the proximity of the loop detection zone. However, video detection frequently only detects the headlights at night so the call is lost if the video detection zone ends just a few feet in front of the stop bar. Due to varying camera angles it is difficult to give an exact distance, but this type of failure 
can be mitigated by drawing the video detectors out in front of the stop bar several feet. However, judgment must be used when extending these detection zones because extending these detection zones often results in left- turning vehicles or pedestrians generating false calls. This problem can also be addressed by lighting the intersection. A test of illuminating the intersection was carried out in late October with the assistance of the Crawfordsville district to verify that illuminating the intersection can address this problem.

\section{Implementation}

Based upon these observations, it is recommended that due to the imprecision of night detection, video detection should not be used to provide dilemma zone protection. The imprecision observed at the stop bar is even worse at the extended distance at which dilemma zone detectors are placed.
When video is used for stop bar detection, special care should be exercised to ensure proper operation. The implementation report of these documents recommends a turn-on procedure that involves both nighttime and daytime inspection of system operation.

\section{Contacts}

For more information:

\section{Prof. Darcy Bullock}

Principal Investigator

Purdue University

1284 Civil Engineering Building

West Lafayette, IN 47907-1284

Phone: 765-494-2226

Fax: 765-496-1105

\section{Prof. Andrzej Tarko}

Principal Investigator

Purdue University

1284 Civil Engineering Building

West Lafayette, IN 47907-1284

Phone: 765-494-5027

Fax: 765-496-1105

\section{Indiana Department of Transportation}

Division of Research

1205 Montgomery Street

P.O. Box 2279

West Lafayette, IN 47906

Phone: 765-463-1521

Fax: $\quad 765-497-1665$

\section{Purdue University}

Joint Transportation Research Program

School of Civil Engineering

West Lafayette, IN 47907-1284

Phone: 765-494-9310

Fax: 765-496-1105 


\section{ACKNOWLEDGEMENT}

This project was carried out with the close cooperation of a number in of Indiana Department of Transportation Personnel. The Study Advisory Committee composed of Ed Cox, Andy Fitzgerald, Ryan Gallagher, Alfredo Hanza, Dennis Lee, Shuo Li, Dan Shamo, Jim Sturdevant, Carl Tuttle, and Kate Weese all provided significant input during the SAC meetings that guided this research. New issues no originally thought of in the origninal proposal related to inductive loop detector counting, video detector counting, and illumination of the intersection were evaluated at their direction.

In addition, Bill Smith, Terry Gowin, Monty Wilson, Steve Gant, and Harry Greer from the Crawfordsville District and Lauri Land from Traffic Support provided tremendous assistance in installing equipment at various phases of the project. Finally, Chip Lang, Mike Lindley and Greg Walk from Traffic Control Corp, and Tad Dickerson, Tom Farr, and Mike Parks from the Traffic Signal Company provided on site support for the installation and calibration of their equipment.

Finally, in November $2001 \mathrm{Jim}$ Sturdevant performed extensive field checks of Autoscope, VideoTrak, and Odectics video detection systems to verify that the results reported in this document were consistent with the performance observed in actual field deployments. 


\section{IMPLEMENTATION REPORT}

Vehicle detectors are important in regulating traffic signals based upon demand, and not on a fixed time. Since the beginning of the use of actuated signals in the 1960's, inductive loops have been the primary detection device used by the Indiana Department of Transportation. The use of video detectors for vehicle detection has recently become more attractive due to advancements in the technology and a drastic reduction in costs. Despite the attractiveness of the technology and the low costs, there is a lack of evaluation methods and procedures for different video detection systems. There is also a need of specialized training for those personnel that will be involved with the deployment and maintenance of video detection systems in the field.

This research directly addresses the need for evaluation methods and procedures by documenting two different methods of video detection evaluation that could be used separately based upon the need, or in conjunction with one another to provide the maximum amount of information. The first of these two methods, or the Discrepancy Method, is accomplished by comparing the individual occupancy times of inductive loop detectors and video detectors for the same traffic flow. The second of the two methods, or the Likelihood Method, involves finding the likelihood that a certain type of discrepancy between inductive loop detectors and video detectors will occur; then finding the likelihood that inductive loop detectors do not indicate presence correctly; and finally, combining these two likelihoods to find the likelihood that video detectors do not indicate vehicle presence correctly under certain conditions. 
The Discrepancy Method of evaluation would allow for training INDOT technicians how to best calibrate a video detector in order to emulate the performance of inductive loop detectors. This could be accomplished by first instructing the technicians on the best practice of installing and setting up video detection systems. The technicians could then draw video detection zones onto the screen in the lab and download these into the video detectors. Once these zones have been set, data could be recorded for approximately thirty minutes or less, and then the data could be examined. If there are problems, the video detectors could be rearranged, and another data collection could be made, and the process repeated until the optimal performance of the video detectors is reached.

The Likelihood Method of evaluation allows one to calibrate a statistical model that tells under which conditions a certain video detection performs the best, and under which conditions it performs poorly. This information is important in deciding which video detection system to employ.

Based upon this work, nighttime detection appears to be of the most concern. Two types of problems were observed:

- The effective length of the detection zone increased from an average of $23.7 \mathrm{ft}$ during the day to an average of $67.7 \mathrm{ft}$ at night. Figure 6-34 and Figure 6-35 document this phenomenon. This has a neglible impact on safety. However, signals operate less efficiently at night because they do not gap out when they should.

- Lost detection when vehicles pull past the stop bar. Figure 7-3 documents this phenomenon. Loop detectors typically do not lose a call in these situations, because the back of the vehicle is still in the proximity of the loop detection zone. However, video detection frequently only detects the headlights at night so the call is lost if the video detection zone ends just a 
few feet in front of the stop bar. Due to varying camera angles it is difficult to give an exact distance, but this type of failure can be mitigated by drawing the video detectors out in front of the stop bar several feet. However, judgment must be used when extending these detection zones because extending these detection zones often results in left turning vehicles or pedestrians generating false calls. This problem can also be addressed by lighting the intersection. Figure 7-8 documents the effectiveness of adding a 400-Watt High Pressure Sodium Light.

Based upon these observations, it is recommended that due to the imprecision of night time detection (Figure 6-33 and Figure 6-34), video detection should not be used to provide dilemma zone protection. The imprecision observed at the stop bar is even worse at the extended distance at which dilemma zone detectors are placed.

In addition, it is recommended that the turn on testing procedure at all new installations encompass the following to ensure that vehicle calls are not lost by video detectors during low volume at night on detectors do not have an unacceptably high false detection rate:

- Pick a low volume time to test - say 4am.

- Ensure that no other vehicles are in the vicinity of the signal.

- Drive test car into lane being evaluated. Via radio, the inspector at the cabinet shall communicate to the driver when the detector being evaluated turns on and turns off.

- Confirm that detection is registered by video detector when vehicle approaches detection zone.

- Creep car forward until detection is lost. Record how many ft the front bumper was past the stop bar. Record in acceptance notes and note on cabinet plans.

- Repeat process for all lanes with video detection. 
- After nighttime test and adjustments are complete, conduct daytime test to ensure that left turning vehicles and pedestrians are not causing an unacceptably high rate of false calls. Note, it is important that this daytime test follow the nighttime testing.

Also, recent tests by INDOT have found that cameras may move due to wind or other external issues. Care shall be taken to inspect video detection systems at regular intervals to ensure the camera has not moved.

Finally, although not directly part of the scope of this project, the instrumented intersection at Northwestern and Stadium provided a mechanism for conducting the count detector tests documented in Appendix A. Based upon the results of the final tests, the Reno detector demonstrated as good if not better performance than the current "fourth loop" for counting cars. If multiple vendors can achieve similar levels of performance, consideration should be given to abandoning the practice of wiring the "fourth loop" to a different detection channel and instead use the new count detector cards.

A last minute addition to this study was to evaluate the accuracy of counts with video detection systems. Appendix B contains a memorandum explaining the procedure used and suggests that turning movement counts obtained from video detection systems are not sufficiently accurate for designing signal timings. 
TABLE OF CONTENTS

Description

Page

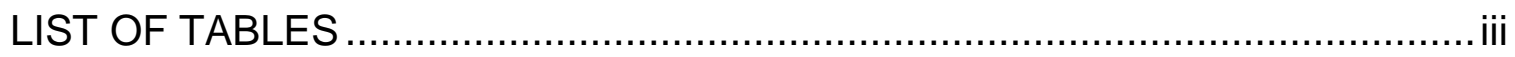

LIST OF FIGURES ..................................................................................

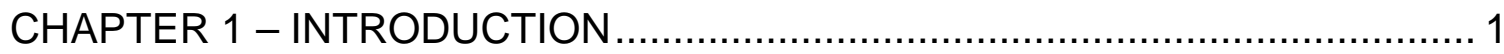

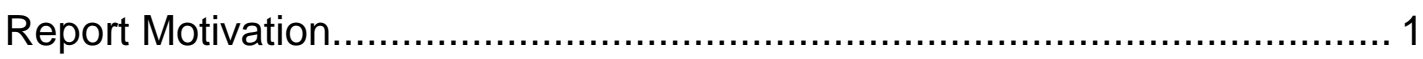

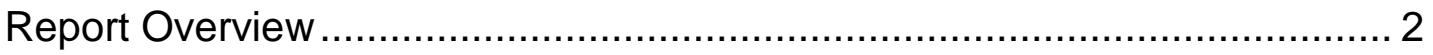

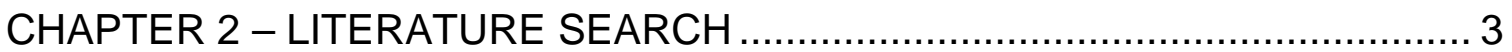

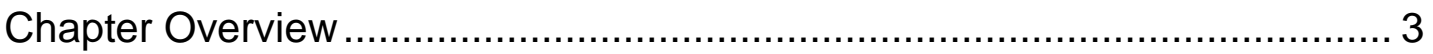

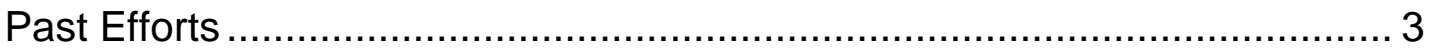

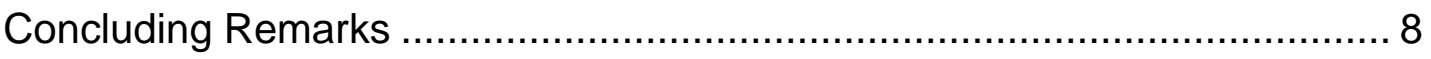

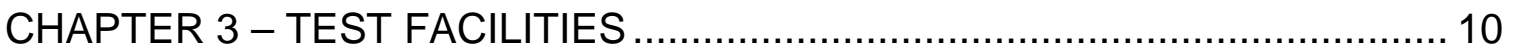

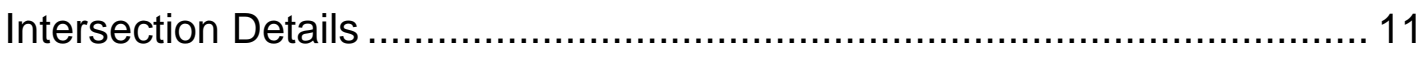

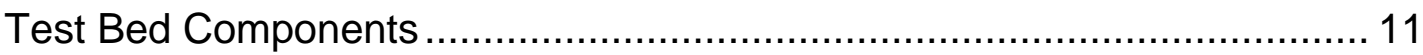

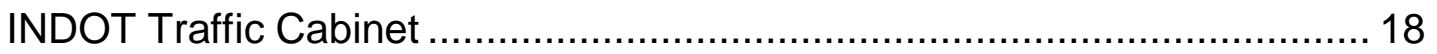

Purdue University Traffic Cabinet ......................................................... 19

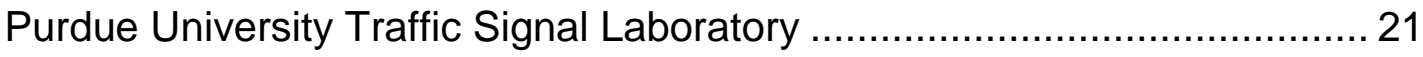

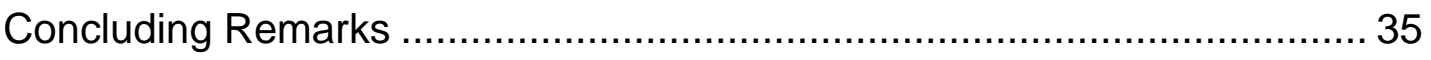

CHAPTER 4 - CONCEPTS OF VIDEO EVALUATION .................................... 36

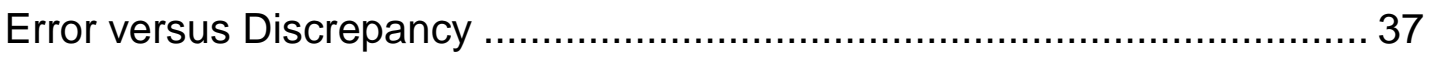

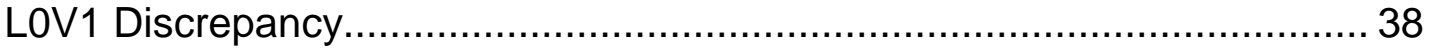

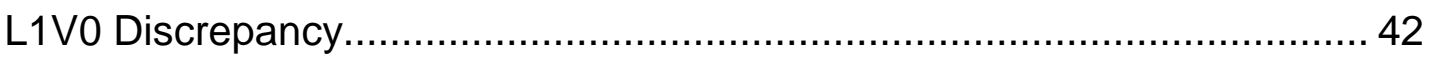

Likelihood of Detection Discrepancies ...................................................... 45

Likelihood of Detection Errors................................................................. 47

Inductive Loop Detector Errors ............................................................ 47

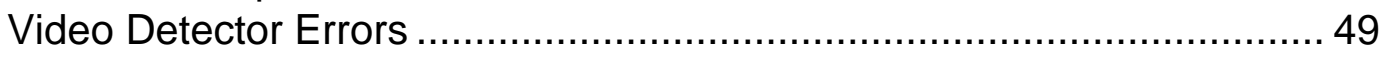

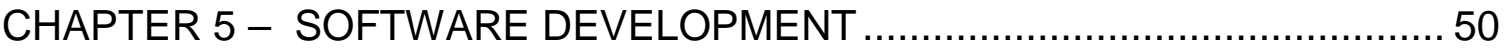

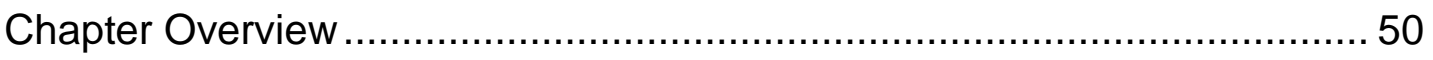

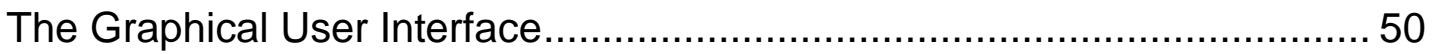

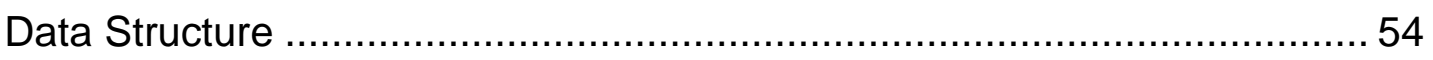

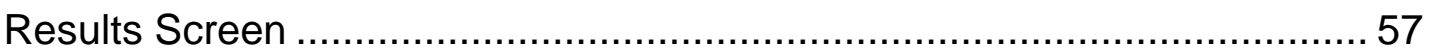




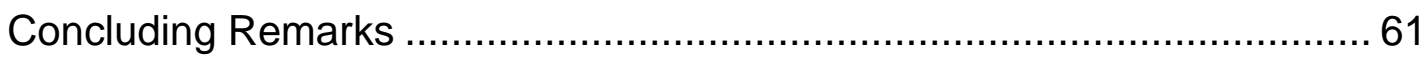

CHAPTER 6 - ANALYSIS OF LOV1 AND L1VO DISCREPANCIES .................62

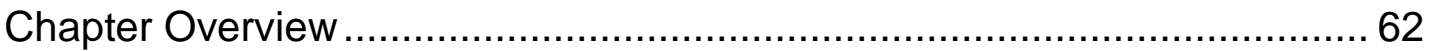

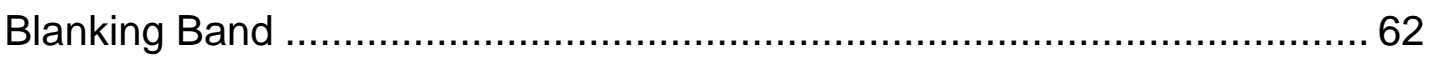

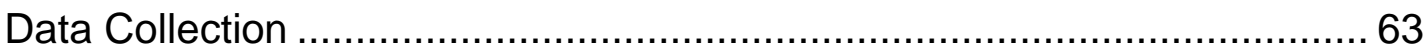

Night Rain Conditions (Before Recalibration) ......................................... 64

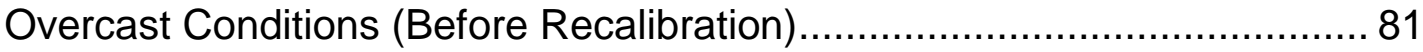

Partly Sunny Conditions (After Recalibration).......................................... 98

Mostly Sunny Conditions (After Installation of New System 2 Cameras)... 115

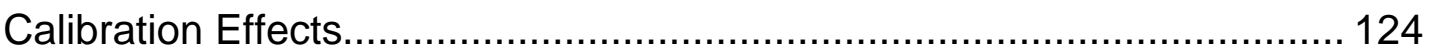

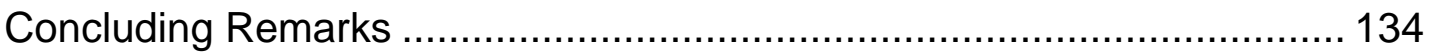

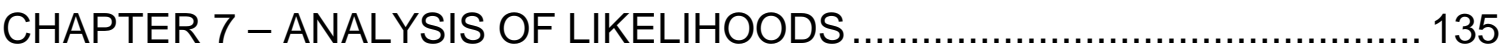

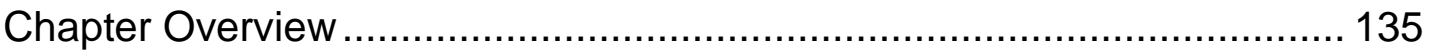

Discrepancies Between Video Detection and Loop Detection ................... 136

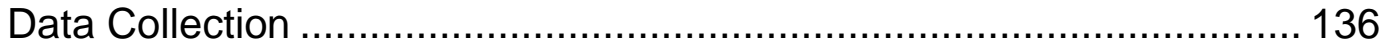

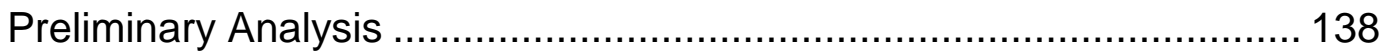

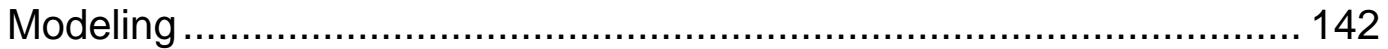

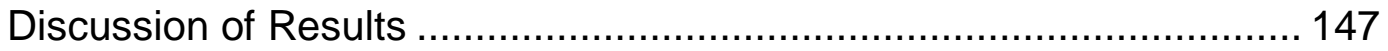

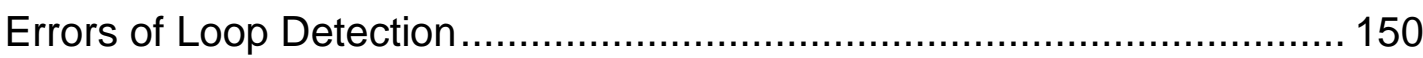

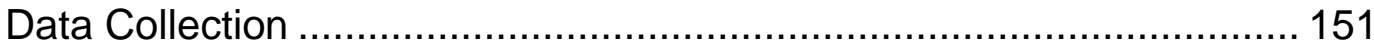

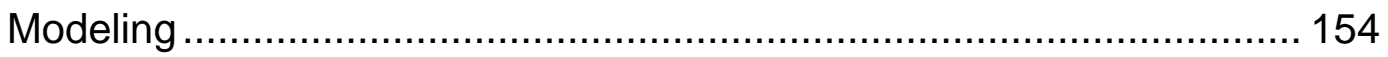

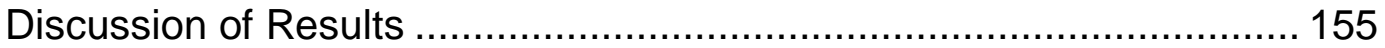

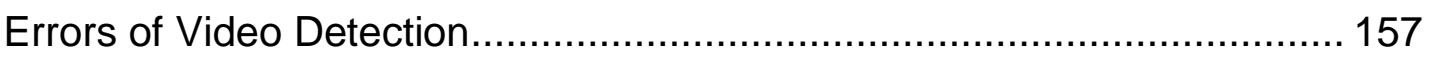

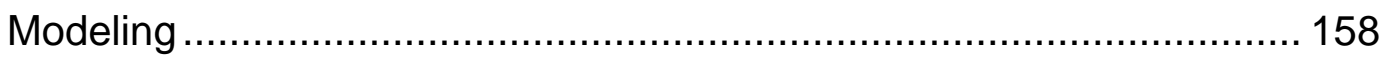

Example Profiles of Video Detection Error Likelihoods........................ 159

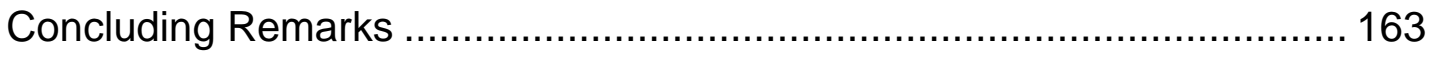

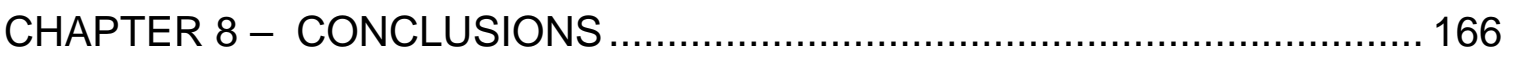

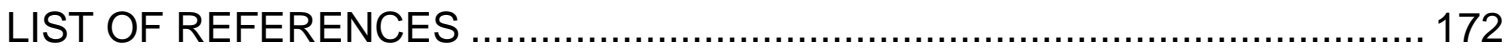

APPENDIX A - SUMMARY OF COUNT DETECTOR EVALUATION ....... 174

APPENDIX B - Evaluation of Video Detection Count Accuracy ................ 189 


\section{LIST OF TABLES}

Table

Table 3-1: Equipment Vendors and Part Numbers

Table 4-1: Comparison Between Inductive Loop and Video Detectors

Table 6-1: Hourly Discrepancy Totals for Phase 1, Blanking Band = $2 \mathrm{sec}$, February 23 \& 24, 2001, Rain

Table 6-2: Hourly Discrepancy Totals for Phase 2, Blanking Band = $2 \mathrm{sec}$, February 23 \& 24, 2001, Rain

Table 6-3: Hourly Discrepancy Totals for Phase 3, Blanking Band = 2 sec.,

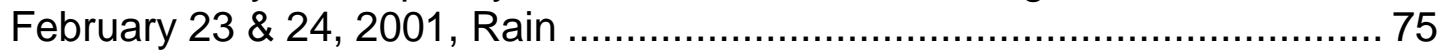

Table 6-4: Hourly Discrepancy Totals for Phase 4, Blanking Band = 2 sec.,

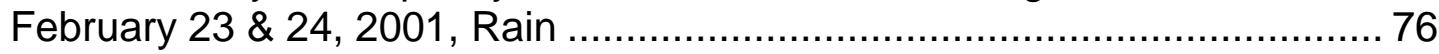

Table 6-5: Hourly Discrepancy Totals for Phase 5, Blanking Band = $2 \mathrm{sec}$.,

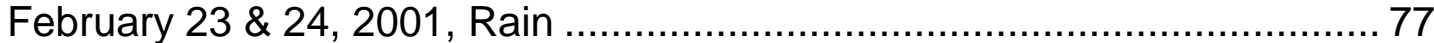

Table 6-6: Hourly Discrepancy Totals for Phase 6, Blanking Band = 2 sec.,

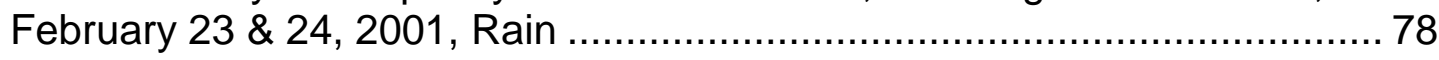

Table 6-7: Hourly Discrepancy Totals for Phase 7, Blanking Band = 2 sec.,

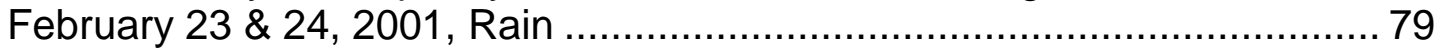

Table 6-8: Hourly Discrepancy Totals for Phase 8, Blanking Band = 2 sec.,

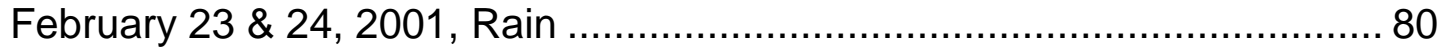

Table 6-9: Hourly Discrepancy Totals for Phase 1, Blanking Band $=2 \mathrm{sec}$,

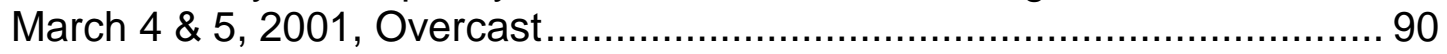

Table 6-10: Hourly Discrepancy Totals for Phase 2, Blanking Band $=2 \mathrm{sec}$, March 4 \& 5, 2001, Overcast............................................................... 91

Table 6-11: Hourly Discrepancy Totals for Phase 3, Blanking Band = 2 sec., March 4 \& 5, 2001, Overcast........................................................... 92

Table 6-12: Hourly Discrepancy Totals for Phase 4, Blanking Band = 2 sec., March 4 \& 5, 2001, Overcast................................................................ 93

Table 6-13: Hourly Discrepancy Totals for Phase 5, Blanking Band = 2 sec., March 4 \& 5, 2001, Overcast..... 
Table 6-14: Hourly Discrepancy Totals for Phase 6, Blanking Band = 2 sec.,

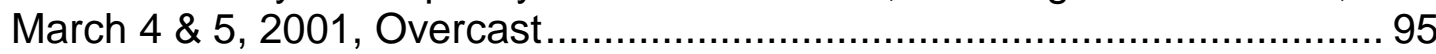

Table 6-15: Hourly Discrepancy Totals for Phase 7, Blanking Band = 2 sec.,

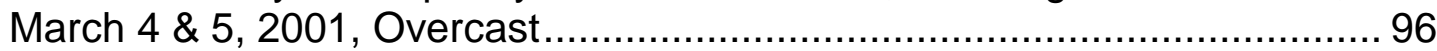

Table 6-16: Hourly Discrepancy Totals for Phase 8, Blanking Band = $2 \mathrm{sec}$., March 4 \& 5, 2001, Overcast.....

Table 6-17: Hourly Discrepancy Totals for Phase 1, Blanking Band $=2 \mathrm{sec}$, April 2 \& 3, 2001, After Recalibration, Partly Sunny ................................ 107

Table 6-18: Hourly Discrepancy Totals for Phase 2, Blanking Band $=2 \mathrm{sec}$, April 2 \& 3, 2001, After Recalibration, Partly Sunny .................................. 108

Table 6-19: Hourly Discrepancy Totals for Phase 3, Blanking Band = 2 sec., April 2 \& 3, 2001, After Recalibration, Partly Sunny ................................ 109

Table 6-20: Hourly Discrepancy Totals for Phase 4, Blanking Band = 2 sec., April 2 \& 3, 2001, After Recalibration, Partly Sunny ...

Table 6-21: Hourly Discrepancy Totals for Phase 5, Blanking Band $=2 \mathrm{sec}$., April 2 \& 3, 2001, After Recalibration, Partly Sunny

Table 6-22: Hourly Discrepancy Totals for Phase 6, Blanking Band = $2 \mathrm{sec}$., April 2 \& 3, 2001, After Recalibration, Partly Sunny

Table 6-23: Hourly Discrepancy Totals for Phase 7, Blanking Band = 2 sec., April 2 \& 3, 2001, After Recalibration, Partly Sunny ....

Table 6-24: Hourly Discrepancy Totals for Phase 8, Blanking Band = 2 sec., April 2 \& 3, 2001, After Recalibration, Partly Sunny

Table 6-25: 24 Hour Discrepancy Totals (in seconds) Before Recalibration, Blanking Band $=2 \mathrm{sec}$.

Table 6-26: 24 Hour Discrepancy Totals (in seconds) After Recalibration, Blanking Band $=2 \mathrm{sec}$.

Table 6-27: Hourly Discrepancy Totals for Phase 1, Blanking Band $=2 \mathrm{sec}$, September 26 \& 27, 2001, Mostly Sunny, New Cameras 120

Table 6-28: Hourly Discrepancy Totals for Phase 3, Blanking Band $=2 \mathrm{sec}$, September 26 \& 27, 2001, Mostly Sunny, New Cameras

Table 6-29: Hourly Discrepancy Totals for Phase 6, Blanking Band $=2 \mathrm{sec}$, September 26 \& 27, 2001, Mostly Sunny, New Camera

Table 6-30: Hourly Discrepancy Totals for Phase 8, Blanking Band $=2$ sec., September 26 \& 27, 2001, Mostly Sunny, New Camera .

Table 6-31: 24 Hour Discrepancy Totals (in seconds) After New System 2 Camera Installation on Eastbound and Southbound Approaches, Blanking Band $=2 \mathrm{sec}$ 
Table 6-32: Summarization of Video Detection Activation Distances (ft) 132

Table 6-33: Increase in Video Detection Activation Distances from Day to Night Due to Headlights. 133

Table 6-34: Allowed Gap (seconds) Provided by Various Loop Length/Approach Speed Combinations (Orcutt 1993). 133

Table 7-1: Characteristics That Possibly Effect Video and Loop Detection Performance. 136

Table 7-2: Conditions Represented in Sample 138

Table 7-3: L1V0 Likelihood Model for System 1 ........................................ 145

Table 7-4: LOV1 Likelihood Model for System 1 ......................................... 145

Table 7-5: L1V0 Likelihood Model for System 2 ......................................... 146

Table 7-6: LOV1 Likelihood Model for System 2 ............................................ 146

Table 7-7: Sensitivity of the L1V0 Likelihood Model for System 1 .................. 147

Table 7-8: Sensitivity of the LOV1 Likelihood Model for System 1 .................. 147

Table 7-9: Sensitivity of the L1V0 Likelihood Model for System 2 .................. 148

Table 7-10: Sensitivity of the LOV1 Likelihood Model for System 2 ................ 148

Table 7-11: Shift and Adjustment Factor for Ground Truth Time.................... 154

Table 7-13: T1L0 Likelihood Model for Loop Ground Truth ............................ 155

Table 7-14: Sensitivity of the TOL1 Likelihood Model for Loop Ground Truth .. 156

Table 7-15: Sensitivity of the T1L0 Likelihood Model for Loop Ground Truth .. 156

Table A-1: Summary of Reno Detector Performance, October 2000 .............. 178

Table A-2: Summary of 3M Detector Performance, September 2000 ............. 178

Table A-3: Summary of Reno Detector Performance, February 2001 .............. 179 


\section{LIST OF FIGURES}

FIGURE

Figure 3-1: Fiber Optic Cable Run from Intersection to Laboratory .................. 10

Figure 3-2: Intersection and Inductive Loop Layout...................................... 13

Figure 3-3: Fixed Base and Focal Length Cameras (NW12 - NW15 in Figure

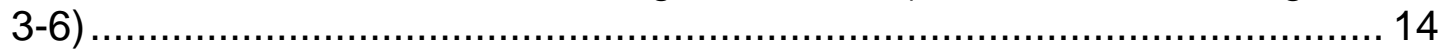

Figure 3-4: Pan-Tilt-Zoom Camera (NW11 in Figure 3-6) ............................... 14

Figure 3-5: Axis PTZ Controller (NW10 in Figure 3-6) .................................. 14

Figure 3-6: Schematic Drawing of Test Bed Components............................... 15

Figure 3-7: INDOT and Purdue Cabinets ..................................................... 16

Figure 3-8: INDOT Traffic Cabinet (NW03) ……….................................... 16

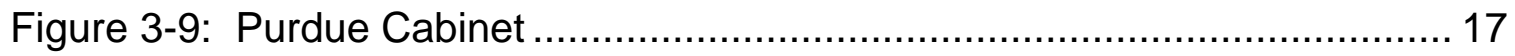

Figure 3-10: Picture of Purdue University Traffic Signal Laboratory, with students

Figure 3-11: Fiber Video Transmitters (NW05 - NW08) ................................... 20

Figure 3-12: Traffic Signal Interface Board in Purdue Cabinet (NW01, NW02) . 20

Figure 3-13: 100 Base-T to 100 Base-FX Media Conversion (NW04, CIVL02). 21

Figure 3-14: Video and CID Interface in the Lab .......................................... 22

Figure 3-15: Video Traffic Detector Cabinet Inside the Lab............................ 23

Figure 3-16: Opto22 I/O Modules - Video Detector Status Interpretation

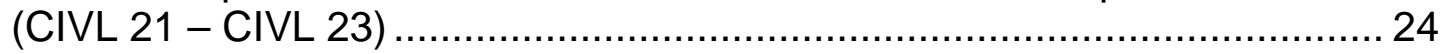

Figure 3-18: Northbound Fields ........................................................... 27

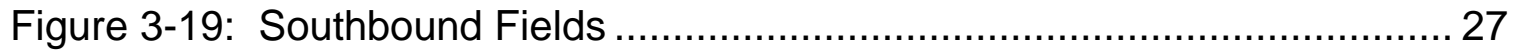

Figure 3-20: Eastbound Fields .............................................................. 28

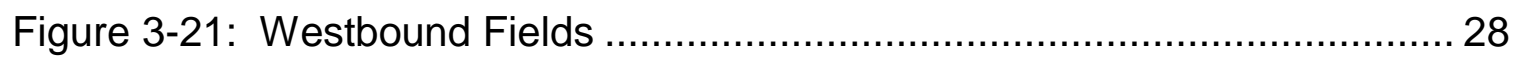

Figure 3-22: Online Video -- Sample Night-time Video with Text Overlay ......... 29

Figure 3-23: Relation of Text Overlay to Detector Layout................................. 30

Figure 3-24: Northbound Detector Layout ...................................................... 31 
Figure 3-25: Southbound Detector Layout.................................................... 32

Figure 3-26: Eastbound Detector Layout...................................................... 33

Figure 3-27: Westbound Detector Layout.................................................. 34

Figure 4-1: Interpretation of LOV1 and L1V0 Discrepancies ........................... 38

Figure 4-2: Example Screen Capture of LOV1 Discrepancy Caused by Headlight Glare in Left Turn Lane (Systems 1 \& 2, Before Recalibration)..................... 39

Figure 4-3: Example Screen Capture of LOV1 Error Caused by Video Detection of Pedestrian (Northbound Left Turn Lane, System 1, After Recalibration).. 41

Figure 4-4: Example Screen Capture of LOV1 Error Caused by Vehicle Shadows (Northbound Through Lane, System 1, After Calibration)............................ 42

Figure 4-5: Example Screen Captures of L1V0 Error Caused by Inductive Loops

(Before Recalibration, System 1)......................................................... 43

Figure 4-6: Example Screen Capture of L1V0 Discrepancy Caused by Poorly Calibrated Video Detector Dropping Detection at Night (Before Recalibration)

Figure 4-7: Example Screen Capture of L1V0 Error Caused by Loops

(Northbound Through Lanes, After Recalibration) ..................................... 45

Figure 4-8: Example Detection Events .................................................... 47

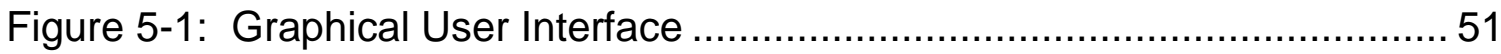

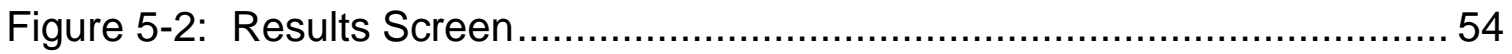

Figure 5-3: Sample Data Collection Times Table …..................................... 55

Figure 5-4: Sample Start and Stop Tables for the Autoscope on the Northbound

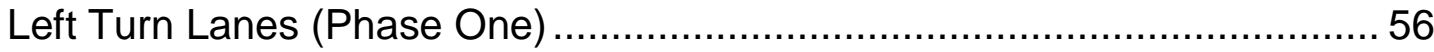

Figure 5-5: Sample Start and Stop Tables for the Video-Trak 905 on the Southbound Left Turn Lanes (Phase One) ............................................. 56

Figure 5-6: Sample Start and Stop Tables for the Inductive Loops on the Southbound Left Turn Lanes (Phase One) ............................................. 57

Figure 5-7: Example of Individual Discrepancies Graph................................. 59

Figure 5-8: Example of Cumulative Discrepancies Graph .............................. 60

Figure 5-9: Example of Likelihood Estimate Graph ........................................ 61

Figure 6-1: Phase 1, Blanking Band $=2$ sec., February $23 \&$ 24, 2001, Rain ... 65

Figure 6-2: Phase 2, Blanking Band $=2$ sec., February $23 \&$ 24, 2001, Rain ... 66

Figure 6-3: Phase 3, Blanking Band $=2$ sec., February $23 \&$ 24, 2001, Rain ... 67

Figure 6-4: Phase 4, Blanking Band $=2$ sec., February $23 \& 24$, 2001, Rain ... 68

Figure 6-5: Phase 5, Blanking Band $=2$ sec., February $23 \& 24$, 2001, Rain ... 69 
Figure 6-6: Phase 6, Blanking Band $=2$ sec., February 23 \& 24, 2001, Rain ... 70

Figure 6-7: Phase 7, Blanking Band $=2$ sec., February $23 \&$ 24, 2001, Rain ... 71

Figure 6-8: Phase 8, Blanking Band $=2$ sec., February $23 \&$ 24, 2001, Rain ... 72

Figure 6-9: Phase 1, Blanking Band $=2$ sec., March 4 \& 5, 2001, Overcast..... 82

Figure 6-10: Phase 2, Blanking Band $=2$ sec., March $4 \&$ 5, 2001, Overcast ... 83

Figure 6-11: Phase 3, Blanking Band $=2$ sec., March $4 \&$ 5, 2001, Overcast ... 84

Figure 6-12: Phase 4, Blanking Band $=2$ sec., March 4 \& 5, 2001 Overcast .... 85

Figure 6-13: Phase 5, Blanking Band $=2$ sec., March 4 \& 5, 2001 Overcast .... 86

Figure 6-14: Phase 6, Blanking Band $=2$ sec., March 4 \& 5, 2001 Overcast .... 87

Figure 6-15: Phase 7, Blanking Band $=2$ sec., March 4 \& 5, 2001 Overcast .... 88

Figure 6-16: Phase 8, Blanking Band $=2$ sec., March 4 \& 5, 2001 Overcast .... 89

Figure 6-17: Phase 1, Blanking Band = 2 sec., April 2 \& 3, 2001, After

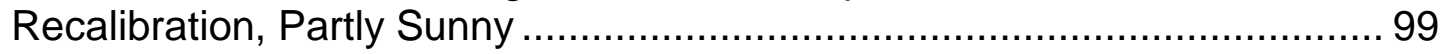

Figure 6-18: Phase 2, Blanking Band = 2 sec., April 2 \& 3, 2001, After

Recalibration, Partly Sunny .............................................................. 100

Figure 6-19: Phase 3, Blanking Band = 2 sec., April 2 \& 3, 2001, After

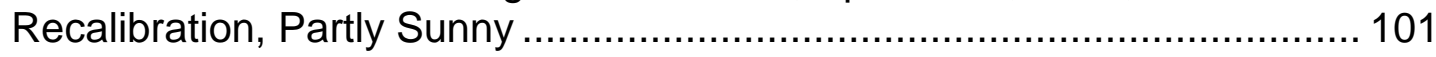

Figure 6-20: Phase 4, Blanking Band = 2 sec., April 2 \& 3, 2001, After

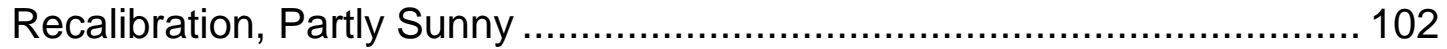

Figure 6-21: Phase 5, Blanking Band = 2 sec., April 2 \& 3, 2001, After

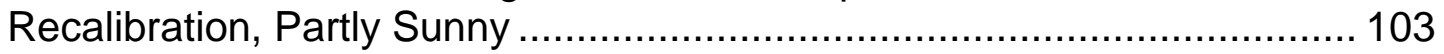

Figure 6-22: Phase 6, Blanking Band = 2 sec., April 2 \& 3, 2001, After

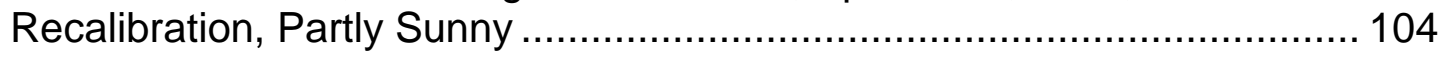

Figure 6-23: Phase 7, Blanking Band = 2 sec., April 2 \& 3, 2001, After

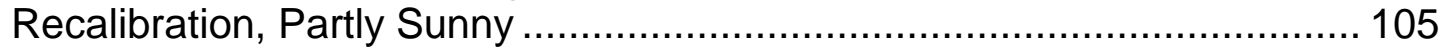

Figure 6-24: Phase 8, Blanking Band = 2 sec., April 2 \& 3, 2001, After

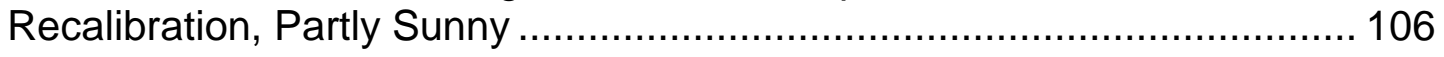

Figure 6-25: Phase 1, Blanking Band = 2 sec., September 26 \& 27, 2001, Mostly Sunny, New Cameras …….............................................................. 116

Figure 6-26: Phase 3, Blanking Band = 2 sec., September 26 \& 27, 2001, Mostly Sunny, New Cameras 117

Figure 6-27: Phase 6, Blanking Band = 2 sec., September 26 \& 27, 2001, Mostly Sunny, New Cameras 118

Figure 6-28: Phase 8, Blanking Band = 2 sec., September 26 \& 27, 2001, Mostly Sunny, New Cameras 119 
Figure 6-29: Distances from Stop Bar on Northbound Approach ................... 125

Figure 6-30: Distances from Stop Bar on Southbound Approach.................... 126

Figure 6-31: Distances from Stop Bar on Eastbound Approach ...................... 126

Figure 6-32: Distances from Stop Bar on Westbound Approach ..................... 127

Figure 6-33: Effect of Headlights on Early Video Detection at Night Before

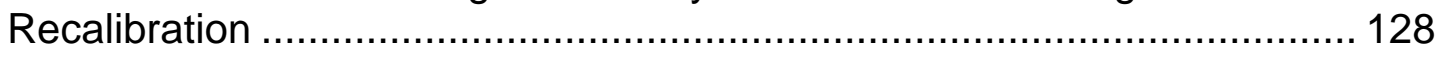

Figure 6-34: Effect of Headlights on Early Video Detection at Night After

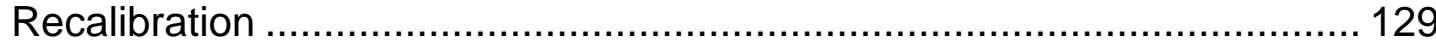

Figure 6-35: Daytime Video Detection Activation Distances After Recalibration

Figure 7-1: System 1 L0V1 Likelihood Profile (Northbound Left Turn Approach, March 4 \& 5, 2001, Overcast)............................................................. 139

Figure 7-3: Example Screen Capture of L1V0 Discrepancy Caused by Headlights Being Dropped by Video Detection .................................................... 142

Figure 7-4: Screen Capture of Northbound Left Turn Lane During a Ground Truth Data Collection.......................................................................... 152

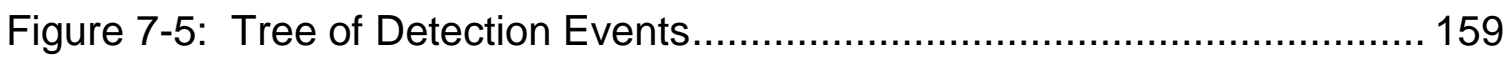

Figure 7-6: System 1 L1V0 vs. T1V0 Likelihood Profile (Northbound Left Turn Approach, March 4 \& 5, 2001, Overcast) ............................................ 160

Figure 7-7: System 1 L0V1 vs. TOV1 Likelihood Profile (Northbound Left Turn Approach, March 4 \& 5, 2001, Overcast) ................................................ 161

Figure 7-8: Test with Southbound approach lighted with 400 Watt High Pressure

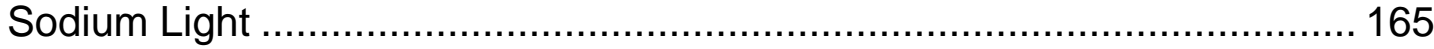

Figure A-1: Intersection and Inductive loop Layout ..................................... 180

Figure A-2: Schematic Drawing of Test Bed Components ............................ 181

Figure A-3: INDOT and Purdue Cabinets ................................................... 182

Figure A-4: INDOT Traffic Cabinet (NW03) ............................................. 182

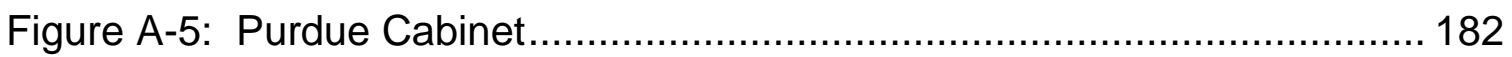

Figure A-6: Video and CID Interface in the Lab....................................... 183

Figure A-7: Relation of text overlay to Northbound detection fields................. 184

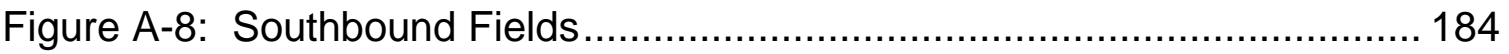

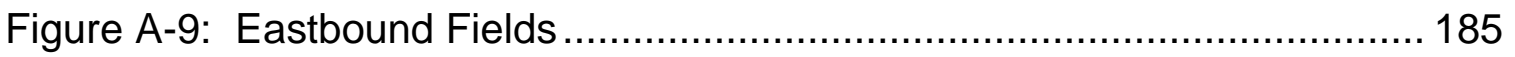

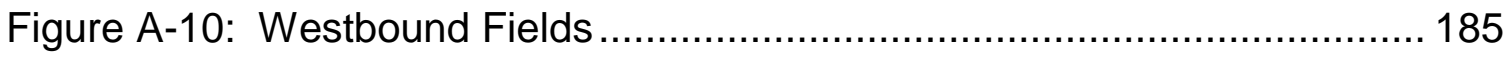

Figure A-11: Signature of one vehicle proceeding across south bound center

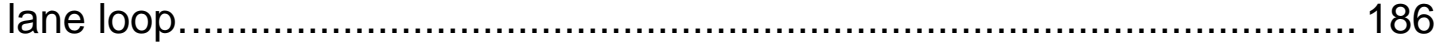


Figure A-12: Signature of one vehicle proceeding across south bound left turn with back loop perhaps missing a turn. .............................................. 186

Figure A-13: Signature of two vehicles proceeding across west bound through movement moving off loop due to snow cover. ...................................... 187

Figure A-14: Signature of one vehicle proceeding across all loops in east bound left turn pocket................................................................................. 187

Figure A-15: Signature of one vehicle proceeding across loops in east bound left turn pocket, but turning before completely traversing the last loop............. 188 


\section{CHAPTER 1 - INTRODUCTION}

Inductive loops have historically been the most favored type of detection device for use at actuated and semi-actuated intersections. Advances in technology, simplified user interfaces, and reduction in cost have recently resulted in relatively wide-scale deployment of video detection technology at signalized intersections. Video detectors also have the distinct advantage of easy relocation and maintenance without disturbing traffic and causing unnecessary delay.

\section{$\underline{\text { Report Motivation }}$}

As video detection becomes more common, there are two important challenges that must be addressed. The first of these challenges is the need for quantitative evaluation procedures for video detectors before they are approved for use. Currently, video detection systems are being deployed without thorough testing. This report will serve to demonstrate quantitative evaluation procedures to evaluate the presence detection ability of video detectors.

The second of the challenges that must be addressed is the training of traffic signal technicians to deploy and maintain video detection systems. This report will not discuss this challenge in detail; however, the test facilities used for the evaluation of video detection systems in this research could be an ideal facility for the training of technicians. 


\section{$\underline{\text { Report Overview }}$}

This report demonstrates the methods and equipment used to perform an evaluation of video detection performance. Chapter 2 is an overview of past research efforts, and also gives a discussion of why these efforts are not ideal for evaluating presence detection at an intersection. Chapter 3 gives a detailed description of the test facilities used to collect data for the evaluation. Chapter 4 gives definitions of the measures of effectiveness that have been defined for this research. Chapter 5 describes the software that was developed to aid in the data reduction and calculations. Chapters 6 and 7 both address the actual evaluation methods and efforts of this research. The last chapter provides some general conclusions and recommendations based on the results obtained from the research. 


\section{CHAPTER 2 - LITERATURE SEARCH}

\section{Chapter Overview}

Several previous research efforts have been conducted to determine a method of evaluating video detection systems. Some of them use inductive loops as the baseline for comparison, and other ones simply use human observation of traffic recorded on videotapes. Using human observation is labor consuming.

The Measures of Effectiveness (MOEs) used to evaluate video detection vary from test to test. Some of the tests use volume and speed comparison to existing inductive loop detectors, and one used accuracy of detection types by human observation. None of the existing research compares the occupancy times of video detectors and inductive loop detectors. This literature review is not completely comprehensive, but it is representative of the different methods that have been used to evaluate video detection.

\section{Past Efforts}

In a study conducted by Cottrell in 1994, the Autoscope 2002 unit was used (Cottrell 1994). It was placed at three different sites on the Capital Beltway. Speed and velocity were the measures that were used to evaluate the video detection systems in this research. The video detectors were drawn over inductive loop detectors in the pavement, and the data from the two detector 
types was compared. The inductive loop detectors were taken to be the ground truth. Data from each detector was downloaded weekly.

In that study, the volume data collected by video detectors was +213 to $+323 \%$ different than the volume recorded by the inductive loops for the same time interval. The corresponding speed data was $-67 \%$ to $-31 \%$ that of the speed collected by the inductive loops. This suggests that the speed measurements reported were much slower than actual, providing that the inductive loops collect the speed data correctly. The results from Sites 1 and 2 were attributed to the placement of the cameras, which was adjacent to the shoulder of the road, and not directly above the roadway (Cottrell 1994).

At Site 3 in the Cottrell study, only 15 minutes of data was evaluated, as there were no inductive loops at this location, and the video data was validated by human observation of the videotapes. At this location, the video cameras were located directly above the lanes that they were monitoring. This placement of the cameras gave a volume comparison of $-4 \%$ relative to human observation. Only the volume comparison was reported for this site, and no speed data was given.

No conclusions were drawn from this report - only suggestions were made to the Virginia Department of Transportation for further study of video detection systems.

In the early 1990's, MacCarley, et al. conducted research in which eight different video detection systems were evaluated for the California Department of Transportation (MacCarley, et al. 1992). The video detection systems evaluated did not all use the same type of detection algorithm. There were two different algorithms for vehicle detection. The Type 1 algorithm establishes two virtual gates a known distance apart within the image. It then measures the amount of 
time difference for a vehicle to change the pixel intensity at the first gate and then at the second gate. The Type 2 algorithm is more complex, and actually tracks the vehicle, determining the velocity. For this reason, the detection systems were broken into two groups before they were evaluated. For the evaluation, the speed and volume measurements from each of the systems were compared. A test suite of 28 different parameters was identified, including variations of camera angle, camera mounting position, departing or arriving traffic, lighting, weather, vibration, electromagnetic noise, and traffic. Data collection was done using videocassette recorders for the tests. Each test segment was 20 minutes in length, with the first 10 minutes allowing the system to cancel out the background and adjust to the ambient light intensity. The data evaluated was all collected from freeway locations.

The result of MacCarley's 1992 evaluation was that neither the Type 1 or Type 2 algorithm proved to be highly superior to the other. Several conditions were determined to cause significant degradation of the detection performance. These were: non-optimum camera placement, day to night transition, headlight reflections on wet pavement, shadows of objects or vehicles outside the detection zone, fog, and heavy rain. It was not uncommon for error rates to be above $20 \%$, and at times $40 \%$ for most of the tests performed.

MacCarley subsequently conducted research using the Vantage Video Traffic Detection System (VTDS), manufactured by Odetics, Inc. (MacCarley 1998). There were four initial objectives in that research, one of which was to assess the performance of the video detection system with respect to accumulated traffic count, average and instantaneous speed, vehicles per unit time per lane, and traffic density. This study was performed at several intersections, each of which was controlled by inductive loops, and not by the video detectors. 
The investigators determined twelve different conditions relating to weather, time of day (sun position), traffic volume, and electromagnetic interference, under which to evaluate the VTDS. They also determined nine different evaluation criteria relating to accurate and non-accurate vehicle presence detection. The ground truth case used for this research was human observation, and therefore the data collections could not be lengthy. Each test case was thirty minutes in length, with the first fifteen minutes allotted for the VTDS to cancel out the background, and the last fifteen minutes for evaluation.

As a result of MacCarley's research, it was reported that $65 \%$ of all vehicles were detected correctly (as they would have been detected by a properly functioning inductive loop), and there was an $8.3 \%$ false detection rate. $64.9 \%$ of all red-green transitions would have been actuated correctly, along with $64.0 \%$ of all green extensions, if the video detectors were the means for actuations of the signals. The system was reported to degrade significantly in the following conditions: transverse lighting, low lighting, night (headlight reflections), rain, shadows, and with vehicles that have a low contrast to the pavement.

Middleton, et al. conducted a study on State Highway 6 in Texas in which the Nestor TrafficVision Video Detector was evaluated with respect to vehicle counts(Middleton et al. 1999). Its ability to record vehicle speeds could not be evaluated because one of the inductive loops that were used as the baseline for the comparison was defective, and with only one loop in the lane, only volumes could be extracted. The camera that was used by the system had an infrared lens to minimize the effect of glare from the sun during the day and headlights at night. Data was collected remotely through telephone lines and ISDN lines. The researchers used LabView software to write some Virtual Instruments (VI) that collected and time-stamped the necessary data using a digital I/O data acquisition card manufactured by National Instruments. 
According to this research, it was reported that the video detector counts were within $5-10 \%$ of the inductive loop counts, except at certain times of the day. At night, the video detectors consistently overcounted by as much as 40 $50 \%$. At around 8:00 am and 6:00 pm, the sun angles cause glare and shadows, causing the video detector to undercount at rates of $10-40 \%$. During heavy rain, the video detectors undercounted by $6-8 \%$. The most consistent period of error was between midnight and 5:00 am, despite the low traffic conditions during these hours of the day.

Middleton and Parker conducted a subsequent study in which the Peek VideoTrak 900 video detection system was used (Middleton and Parker 2000). This study also took place on State Highway 6 in Texas. The camera used by the VideoTrak system was installed 12.2 meters (40 ft.) above the roadway, and 5.8 meters (19 ft.) from the outside lane of the roadway. The two traffic measurement parameters that the VideoTrak was evaluated by was count and speed. Inductive loop detectors in the pavement were used for the baseline count comparison, and a Remote Traffic Microwave Sensor (RTMS) placed over the right southbound travel lane was used for the baseline speed comparison. The RTMS was determined in previous tests to provide reliable and accurate speed measurements. Speeds were not compared on a per-vehicle basis, but instead were compared by the 1-minute interval average speeds reported by the RTMS and the VideoTrak system.

The only weather conditions tested were rain and no rain, because this test was part of an initial evaluation of certain detectors. The largest problem was nighttime conditions, and this was attributed to the fact that there was no road lighting at night, as Peek suggests for installations of its VideoTrak system. Wet pavement during the day or night caused overcounting because of headlight reflections off the pavement. Reduced accuracy was reported during the night 
and times during the day when long vehicular shadows were present. Speed data in particular was erratic in wet weather and at night.

\section{Concluding Remarks}

To date, there are many limitations of those video detection evaluation efforts that have been performed at intersections. In the past, footage of the intersection has been captured on a videocassette tape to be analyzed later. The use of videocassette tapes greatly limits the length of data collection that can be made. Further, the video detection systems are not able to consider the green and red phase indications - information that can improve the detection algorithm performance.

After the video collection is complete, the videocassettes are analyzed by the video detection system, and by human observation. Because the ground truth observations are made by humans, this again limits the amount of time that data can be collected. If too much data were collected, it could never be analyzed, because human observation is so labor intensive.

The following chapter describes test facilities where these issues are addressed and there is very little if any human observation needed. The video detectors and inductive loop detectors are compared to one another in real-time at the time of data collection. All further calculations on the data are accomplished using software that has been developed for that purpose. In addition, as much data can be collected as there is room for storage on the computers being used. Video data is saved using digital video software, which uses approximately $1.8 \mathrm{~GB}$ of storage space for 24 hours of data. The database files are only $18 \mathrm{MB}$ in size for 24 hours of data. With the use of a large hard drive, data collections of over 48 hours are feasible. Once this data has been transferred to compact disks, the hard drive can be cleared, and more video can 
then be saved. The reason for saving the video is that if a problem seems to appear in the calculations, the video with text overlay provides an absolute ground truth. 


\section{CHAPTER 3 - TEST FACILITIES}

The objective of this research is to develop a procedure to evaluate the performance of selected video detection systems. In order to accomplish this objective, a significant effort has gone into assembling a test-bed for video detection. This test-bed is located at the intersection of Northwestern Avenue (US 231) and Stadium Avenue in the city of West Lafayette, Indiana (Figure 3-1).

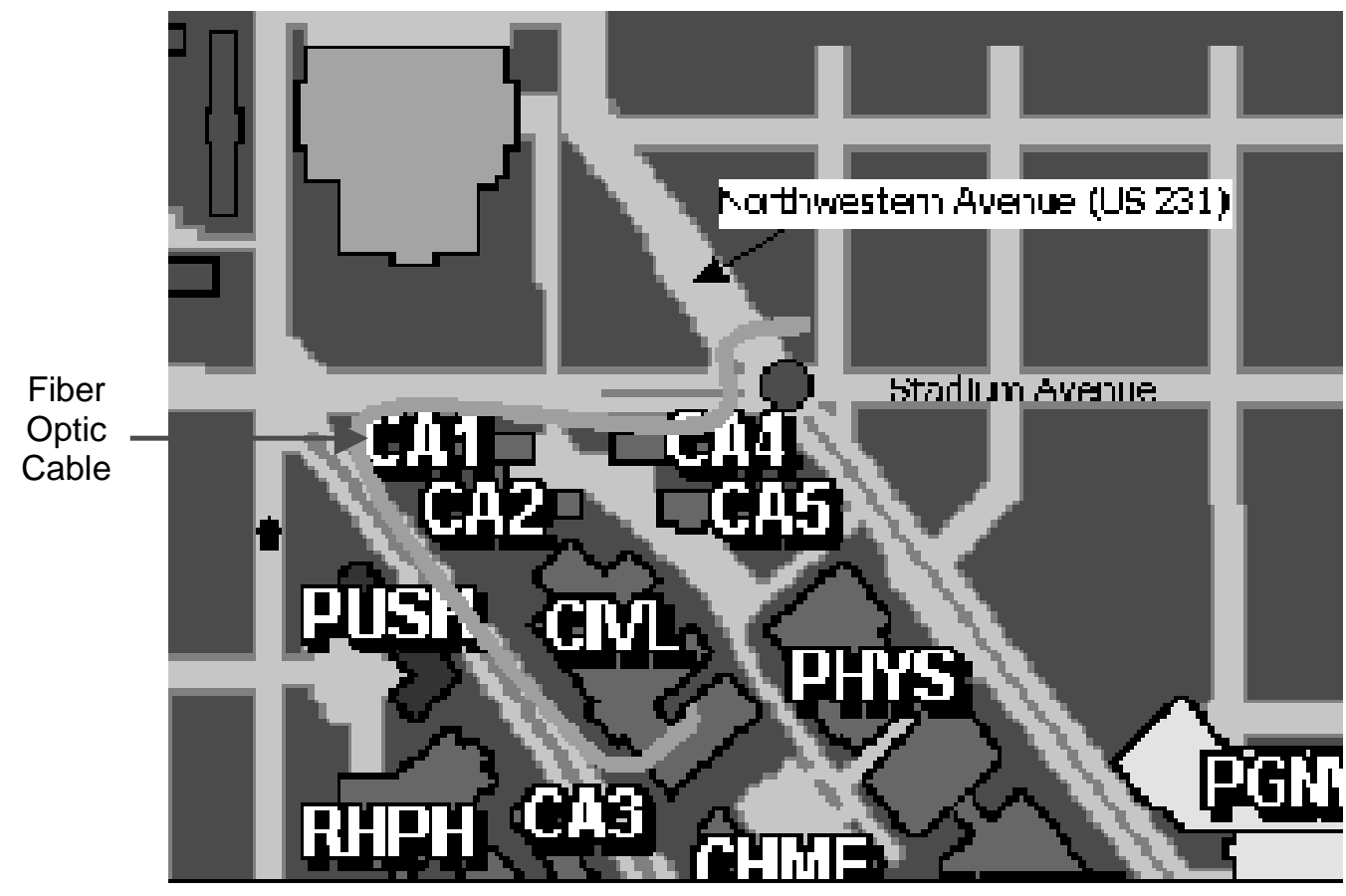

Figure 3-1: Fiber Optic Cable Run from Intersection to Laboratory

This intersection is located on the northeastern side of the Purdue University campus, and is heavily used. The use of this test-bed will allow for many questions about the effectiveness of video detection technologies to be 
answered, and will also be beneficial for training how to most effectively calibrate these systems.

\section{$\underline{\text { Intersection Details }}$}

A drawing of the intersection of Northwestern Avenue (US 231) and Stadium Avenue, along with the detector layout and phase diagram, is shown in Figure 3-2. The reason for which this intersection was used for this research is its heavy volume and close proximity to the Purdue University Civil Engineering building. This close proximity allowed for the easy placement of fiber-optic cables from the intersection to the laboratory inside the building (Figure 3-1). The intersection is a skewed intersection, and also has a great deal of pedestrian traffic. The traffic volume at this intersection is unique in that in addition to the normal morning, noon, and evening peaks, there are also hourly peaks before each class period begins and at major athletic events.

There are five video cameras that are positioned at the intersection, four of which are fixed base and focal length cameras, and one that is a pan-tilt-zoom (PTZ) camera. Figure 3-3 shows the fixed base and focal length cameras, and Figure 3-4 shows the PTZ camera. Figure 3-5 shows the PTZ camera controller.

\section{Test Bed Components}

A schematic drawing with all of the components of the test bed is shown in Figure 3-6. One can see in this figure that there are three main components that comprise the test bed. These are the Indiana Department of Transportation (INDOT) Traffic Cabinet (Figure 3-7 and Figure 3-8), the Purdue University Traffic Cabinet (Figure 3-7 and Figure 3-9), and the Purdue University Traffic Signal Laboratory (Figure 3-10). Each of these components is an integral part of this test bed. 
In Figure 3-6, those hardware components that are out at the intersection are labeled with a prefix of 'NW', which stands for Northwestern [Avenue]. Those hardware components that are inside the Purdue University Traffic Signal Laboratory are labeled with a prefix of 'CIVL', which stands for Civil [Engineering Building]. For a listing of all the vendors and part numbers that were used, see Table 3-1. The three components of the test bed will be more thoroughly described in the following sections of this chapter of the report. 


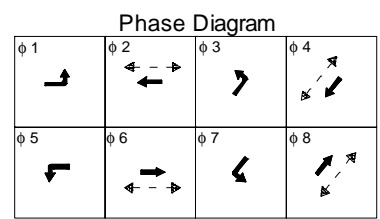

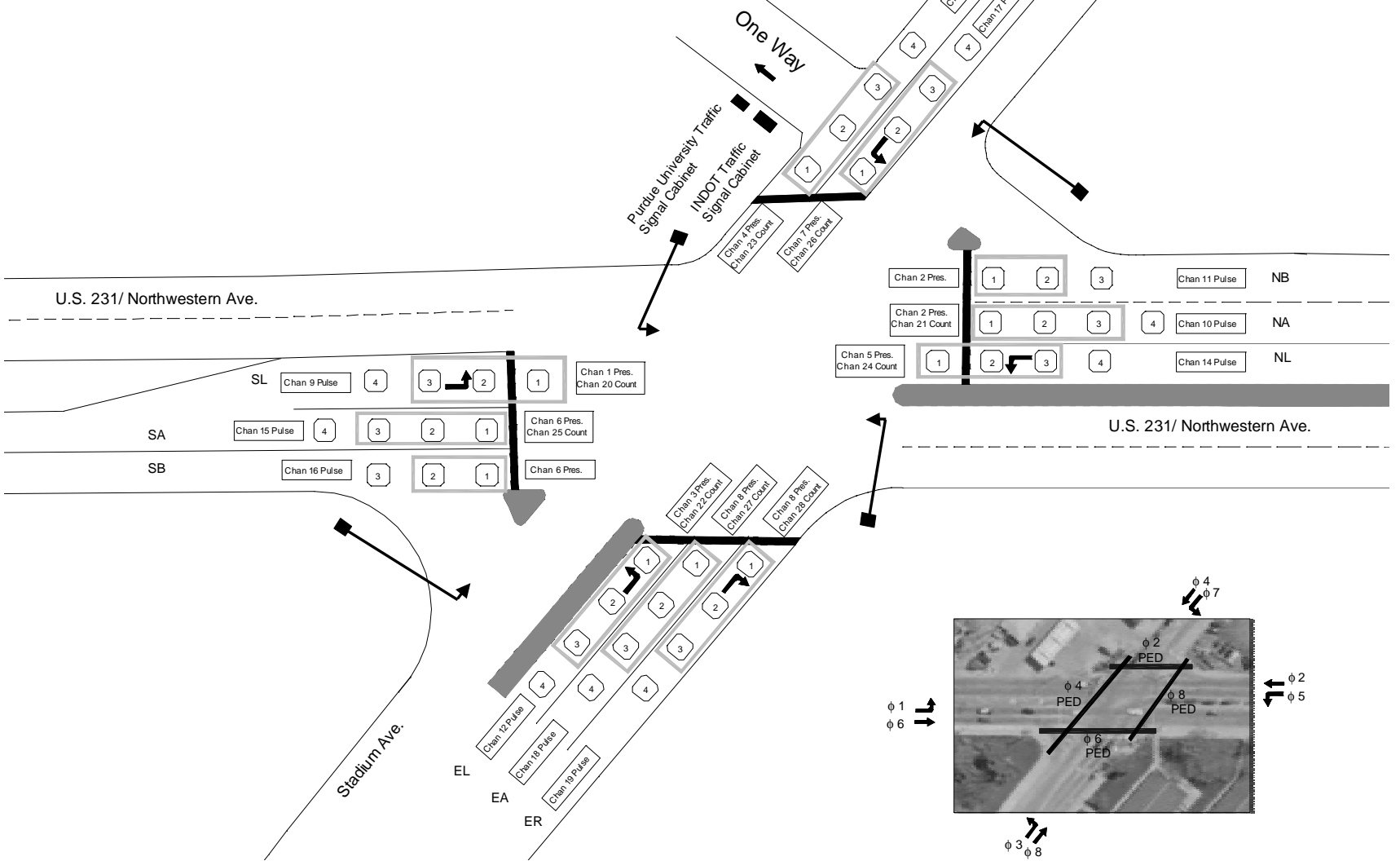

Figure 3-2: Intersection and Inductive Loop Layout 


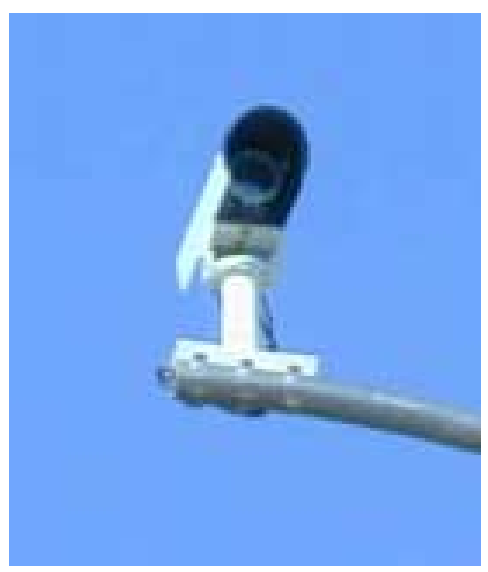

Figure 3-3: Fixed Base and Focal Length Cameras (NW12 - NW15 in Figure 3-6)

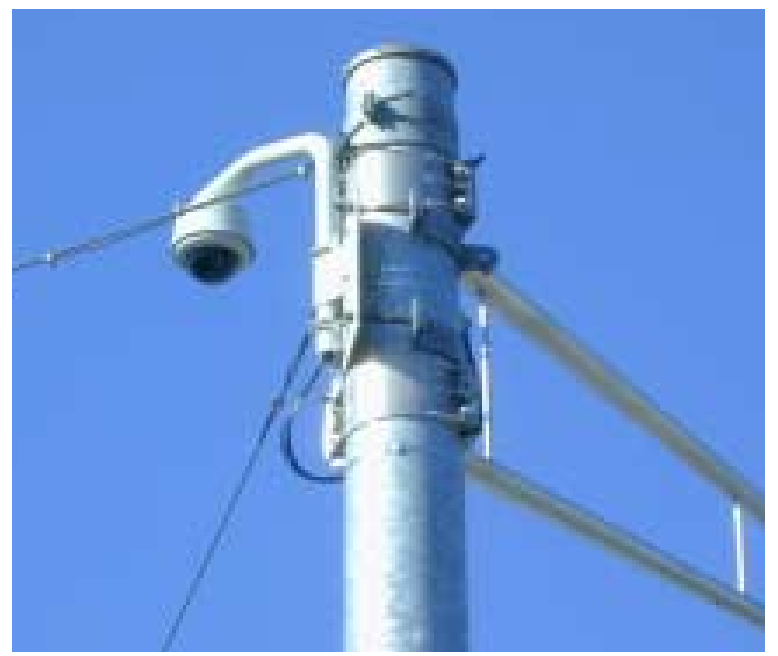

Figure 3-4: Pan-Tilt-Zoom Camera (NW11 in Figure 3-6)

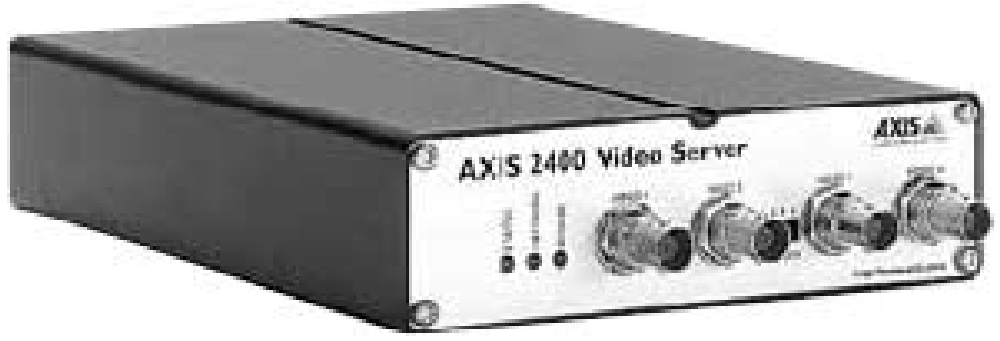

Figure 3-5: Axis PTZ Controller (NW10 in Figure 3-6) 
Figure 3-6: Schematic Drawing of Test Bed Components 


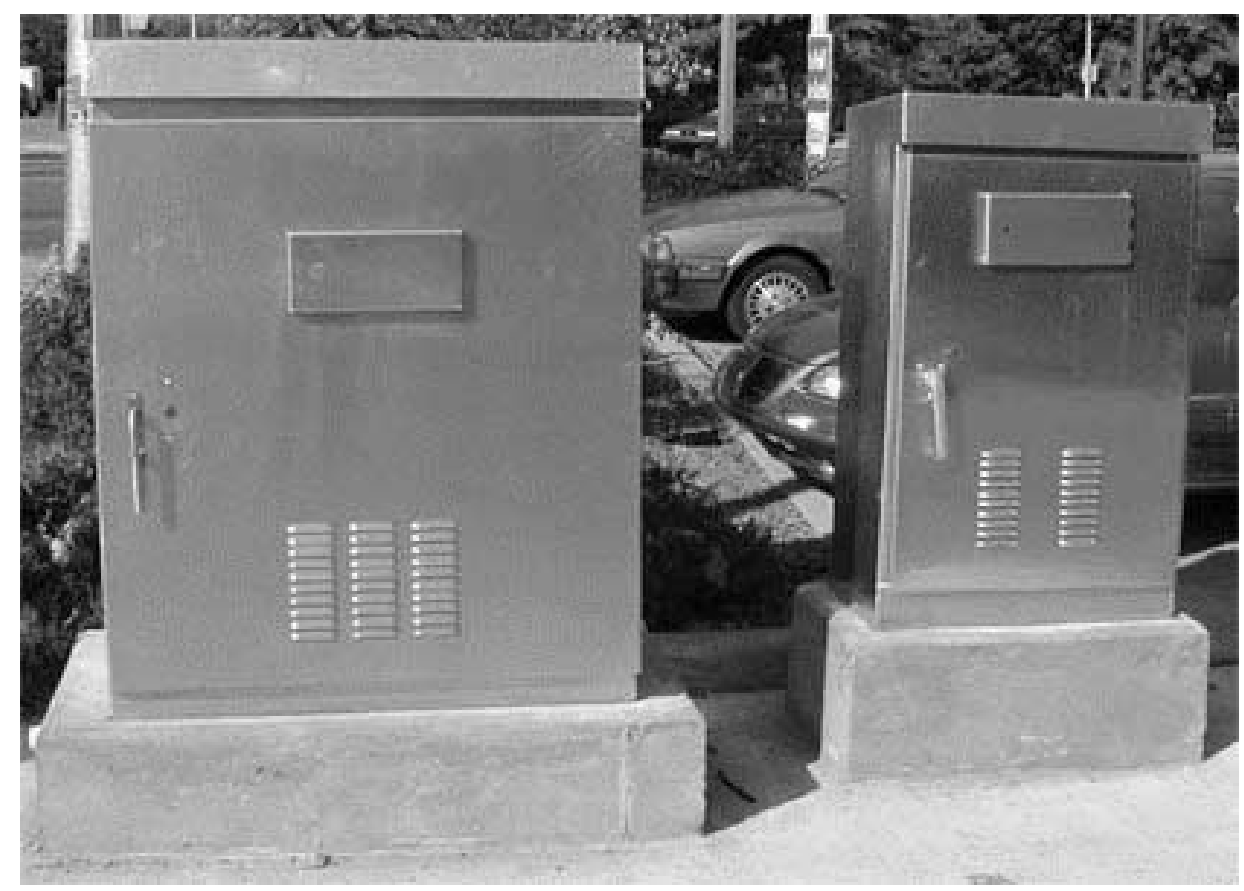

Figure 3-7: INDOT and Purdue Cabinets

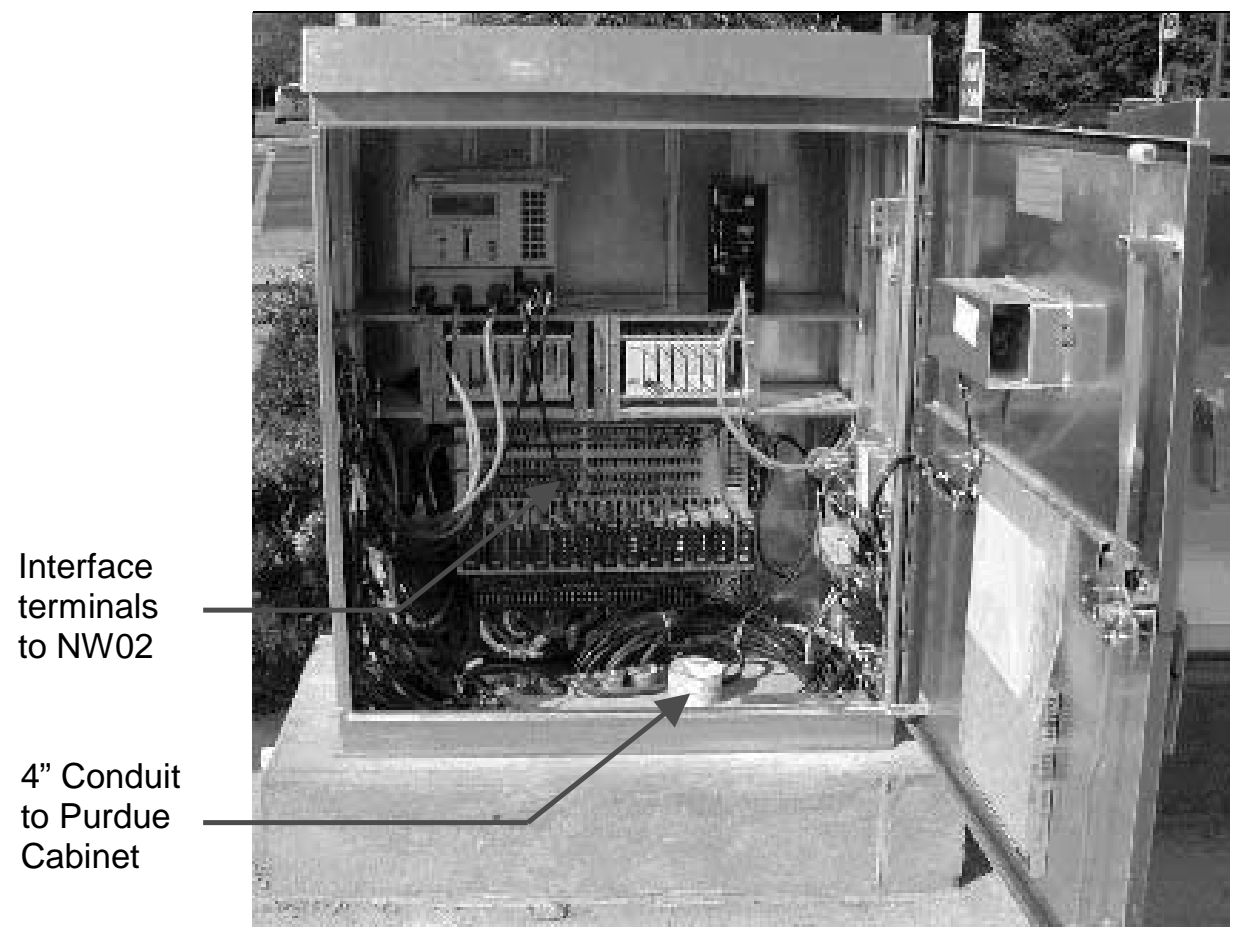

Figure 3-8: INDOT Traffic Cabinet (NW03) 


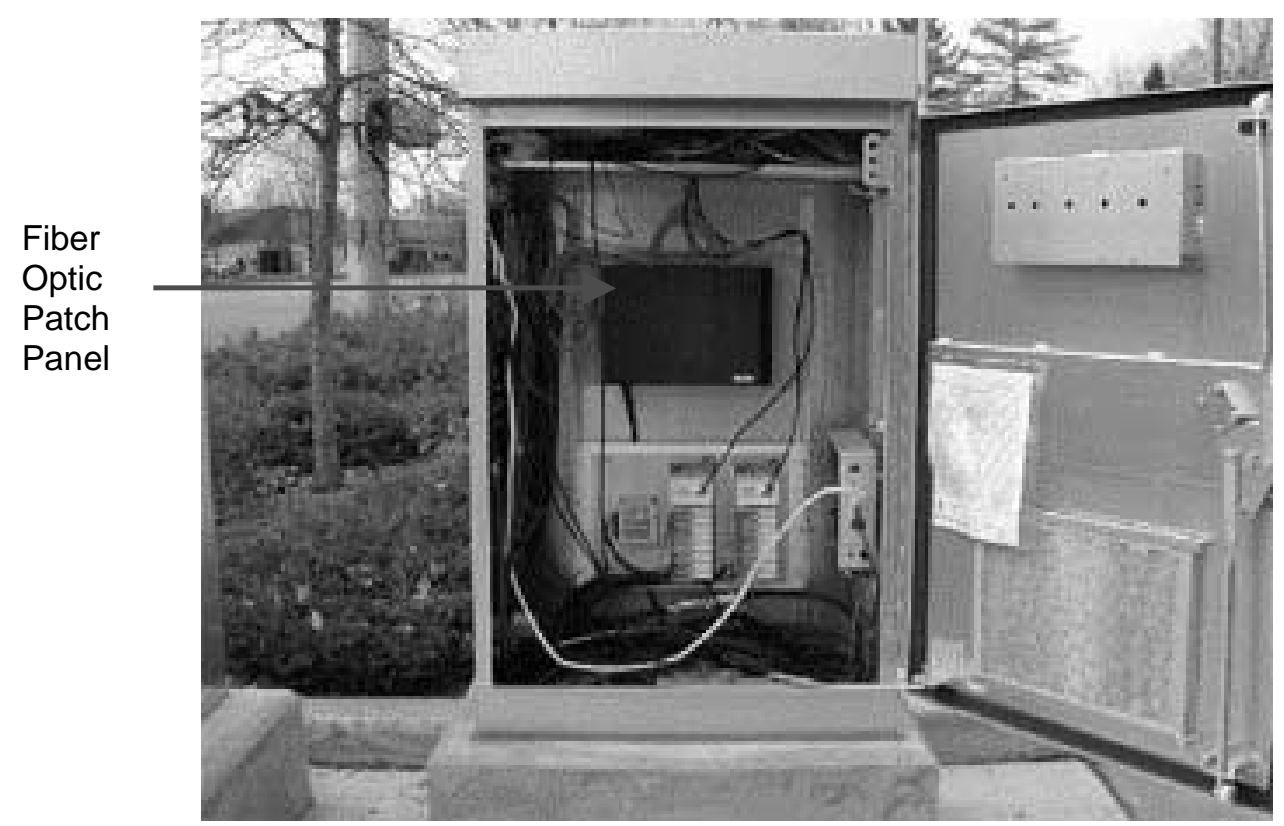

Figure 3-9: Purdue Cabinet

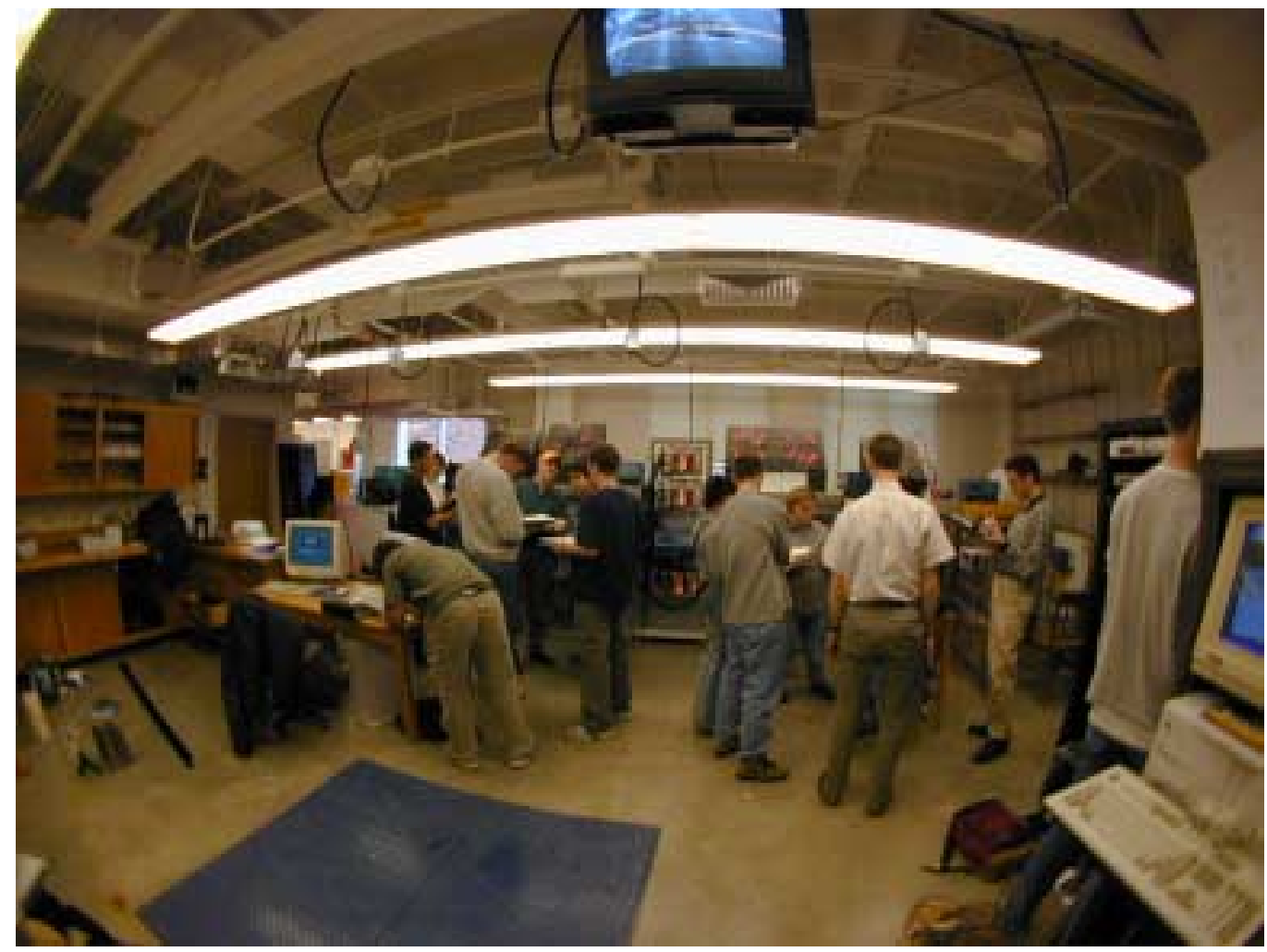

Figure 3-10: Picture of Purdue University Traffic Signal Laboratory, with students 
Table 3-1: Equipment Vendors and Part Numbers

\begin{tabular}{|c|c|c|}
\hline Name & Vendor & ID Number \\
\hline NW01 & OPTO 22 & SNAP-B3000-ENET \\
\hline NW02 & OPTO 22 & SNAP- IDC5 \\
\hline NW03 & Transition Networks & E-PSW--- \\
\hline NW04 & International Fiber & VT4010 \\
\hline NW05- NW09 & Axis & 2400 \\
\hline NW10 & Pelco & SD5AM-PG-E0 \\
\hline NW11 & Econolite & Autoscope Cameras \\
\hline NW12- NW15 & RealNetworks & RealServer \\
\hline CIVL01 & Transition Networks & E-PSW-FX-03 \\
\hline CIVL02 & International Fiber & VR4010 \\
\hline CIVL03-CIVL07 & Linksys & DSSX16E \\
\hline CIVL08 & Philips & LTC 5234 \\
\hline CIVL09 & American Video & VSI-PRO Version 12.00 \\
\hline CIVL10-CIVL13 & OPTO 22 & SNAP-LCM4 \\
\hline CIVL14 & OPTO 22 & SNAP-B3000-ENET \\
\hline CIVL15 & OPTO 22 & SNAP-ODC5 SNK \\
\hline CIVL16 & Econolite & Autoscope \\
\hline CIVL17 & Peek & VideoTrak - 905 \\
\hline CIVL18 & OPTO 22 & SNAP-IDC \\
\hline CIVL21-CIVL22 & ReaINetworks & RealProducer Plus \\
\hline CIVL24-CIVL27 & Philips & LTC2376 \\
\hline CIVL28 & Philips & LTC2017 \\
\hline CIVL29 & Philips & TL24A5T \\
\hline CIVL30 & &
\end{tabular}

$\underline{\text { INDOT Traffic Cabinet }}$

The INDOT traffic cabinet, shown in Figure 3-7 and Figure 3-8, at the location of the test bed contains the normal equipment that one would find at a fully actuated intersection. The only difference that exists can be found on the back panel of the cabinet, which is "tapped" with optically isolated modules for research purposes. This in no way affects the control at the intersection. It merely allows for the vehicular calls placed on all detectors, and the signal states that result from the control of the controller to be replicated inside the lab. The wires that are connected to the back panel of the INDOT cabinet are fed through 
a 4" conduit and into another cabinet that has been placed beside the INDOT cabinet. This cabinet is smaller, and for the purpose of this report, will be called the "Purdue Cabinet."

\section{Purdue University Traffic Cabinet}

The purpose of the Purdue University Traffic Cabinet, shown in Figure 3-7 and Figure 3-9, is to house the fiber video transmitters, shown in Figure 3-11 as NW05 - NW08, and the Controller Interface Devices (CIDs), shown in Figure 3-12 as NW01 and NW02, that recognize the states of detectors and signals coming from the INDOT cabinet. The devices labeled NW01 provide the "brains" of the device, and are linked to a high speed Ethernet link. The devices labeled NW02 are the optically isolated interface modules connected to the INDOT cabinet, NW03. The purpose of the Traffic Signal Interface Board is to read the signals coming from the back panel of the INDOT cabinet. The fiber video transmitters (Figure 3-11, CIVL 05 - CIVL08) turn the video camera signals into a form that can be sent through fiber optic cables into the lab. 


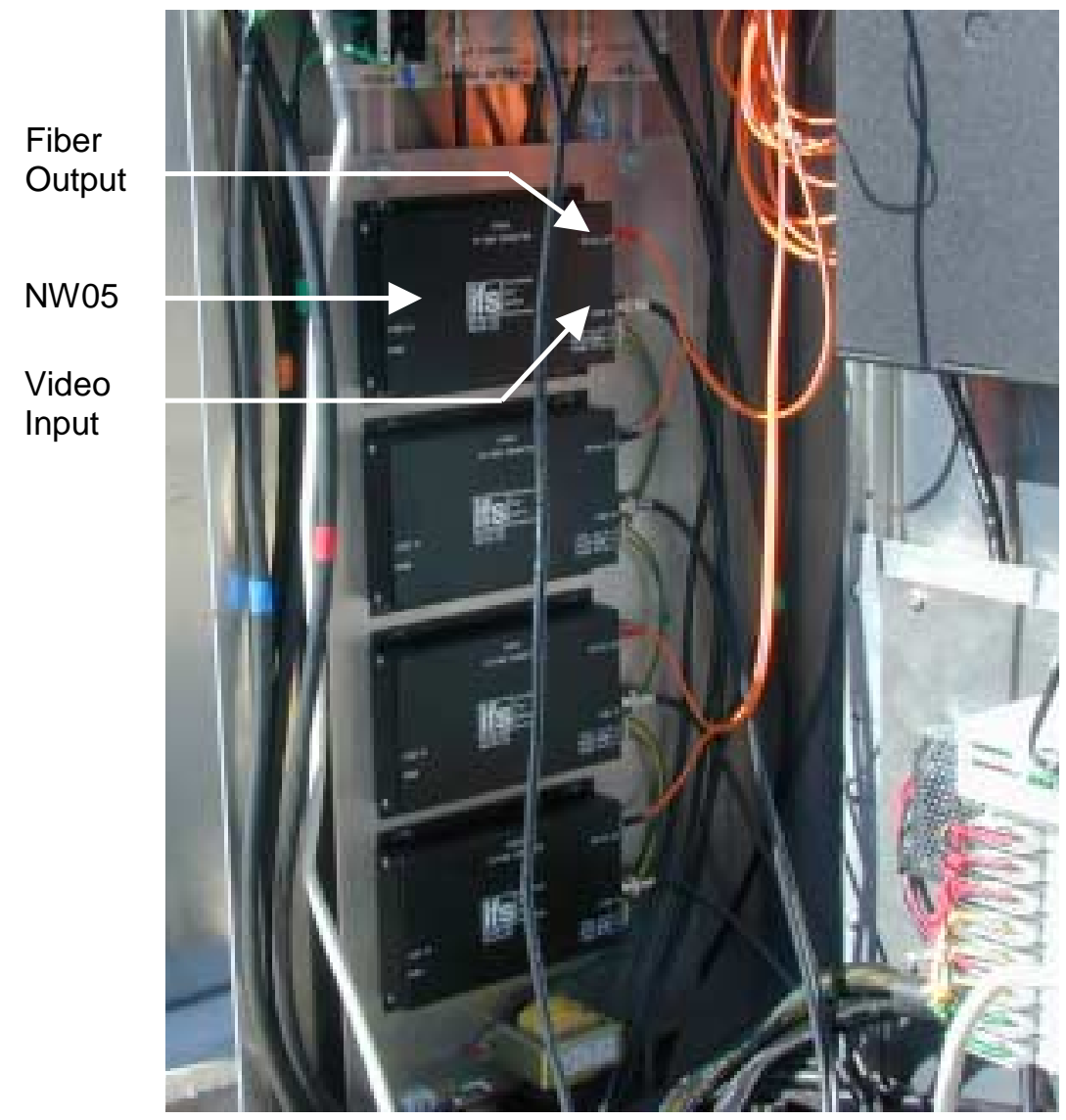

Figure 3-11: Fiber Video Transmitters (NW05 - NW08)

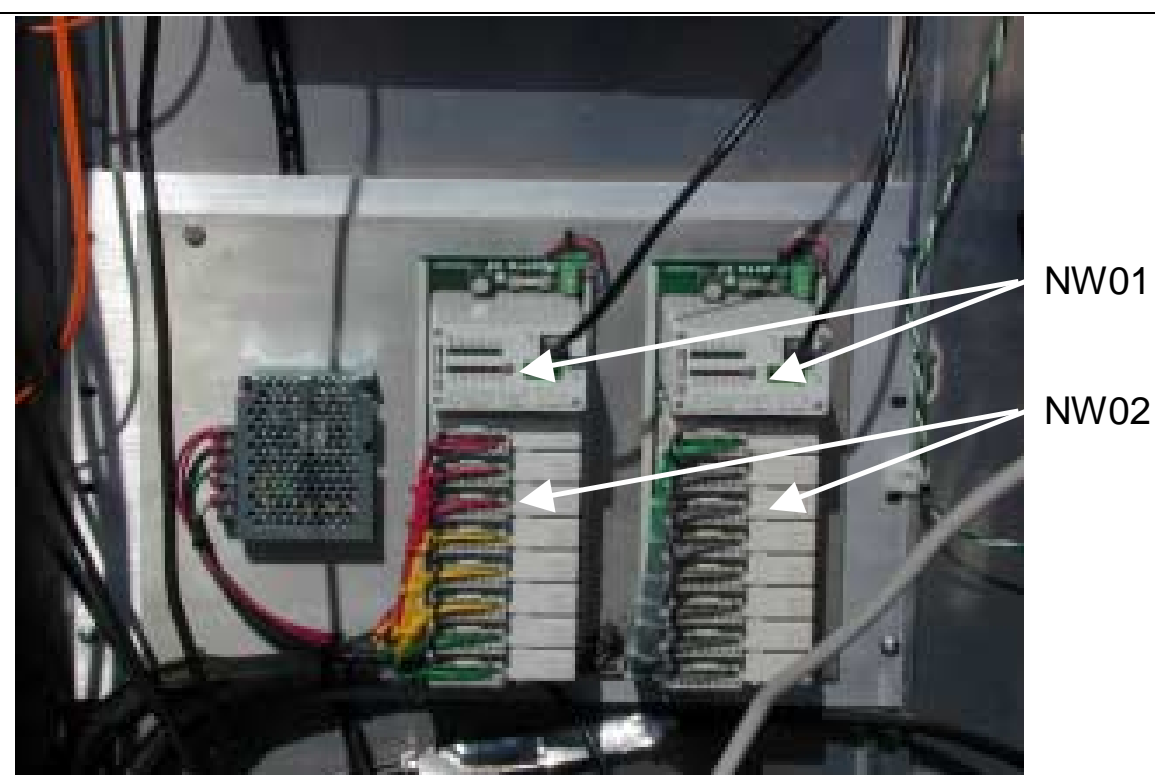

Figure 3-12: Traffic Signal Interface Board in Purdue Cabinet (NW01, NW02) 
Whenever a vehicle activates an inductive loop, or a phase changes in the controller, the CIDs on the traffic signal interface board interpret these signals. These CIDs are fabricated using the Opto22 I/O modules and the Opto22 Ethernet brain. Information received by these modules can be sent over the ethernet via the brain, which has a port for an ethernet cable, and has a small web server. This board is then interfaced with the Traffic Signal Lab with a media converter. Figure 3-13 shows a 100 Base-T to 100 Base-FL Media Converter. This device serves as a "bridge" between the copper wires coming from the traffic signal interface board and the fiber optic cable that allow the information to be relayed to the lab.

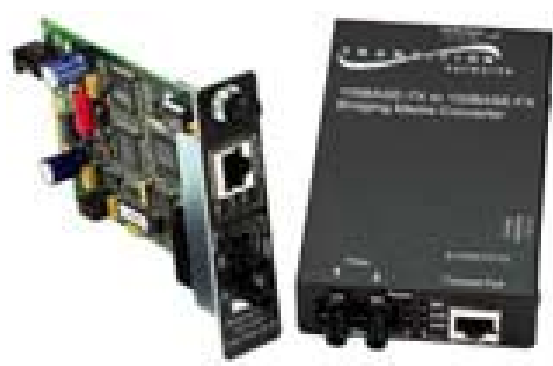

Figure 3-13: 100 Base-T to 100 Base-FX Media Conversion (NW04, CIVL02)

Because this is a dedicated fiber optic cable, the transmission time is negligible from the cabinet at the intersection to the lab inside the Civil Engineering building. Communication regarding the status of the INDOT cabinet (Figure 3-8, NW03) between the lab and the intersection is driven by the Opto22 Controller, shown in Figure 3-14 as CIVL14, which is in the lab.

\section{Purdue University Traffic Signal Laboratory}

The test-bed would not be complete without the equipment that has been assembled in the lab. The fiber-optic cable enters the lab, and is connected to another 100 Base-T to 100 Base-FL media converter (Figure 3-13). This 


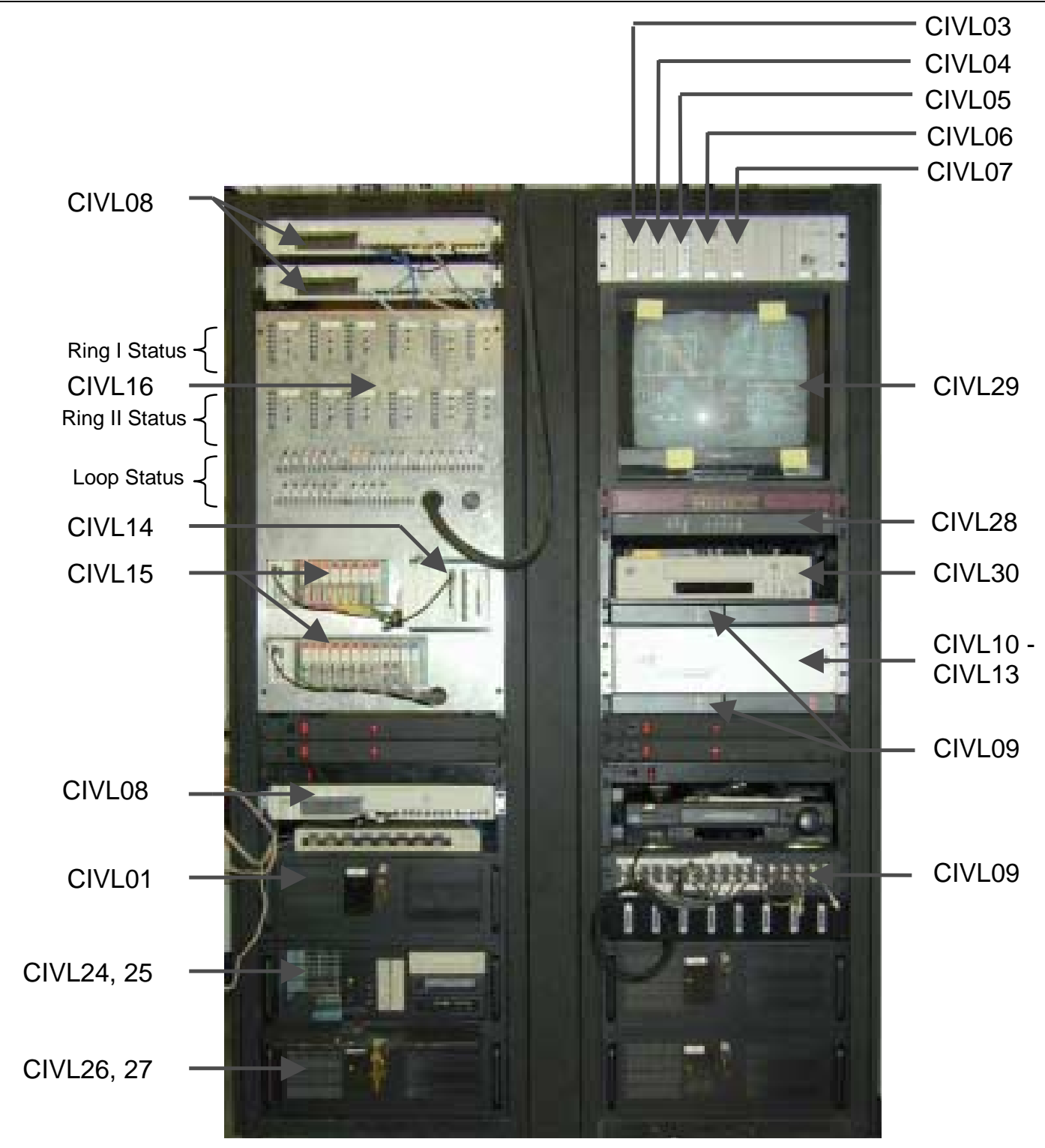

Figure 3-14: Video and CID Interface in the Lab

converts the signal from the fiber optic cable back into a signal that can be sent over copper wire. This media converter is connected to the Ethernet Switch, shown in Figure 3-14 as CIVL08. All the CIDs, as well as the controller, are also hooked up to this switch. This allows for communication between the controller and all the CIDs both inside the lab, and out at the intersection. 
Two racks have been assembled to hold equipment, and a traffic cabinet is also used to house the video detection systems, shown in Figure 3-15. The two video detection systems that are in use at this time are the Econolite Autoscope unit and the Peek VideoTrak-905 unit. Representatives from the respective companies have professionally calibrated these two units so that there should be minimal errors from each. Other video detection systems can easily be introduced to this test bed as the need arises.

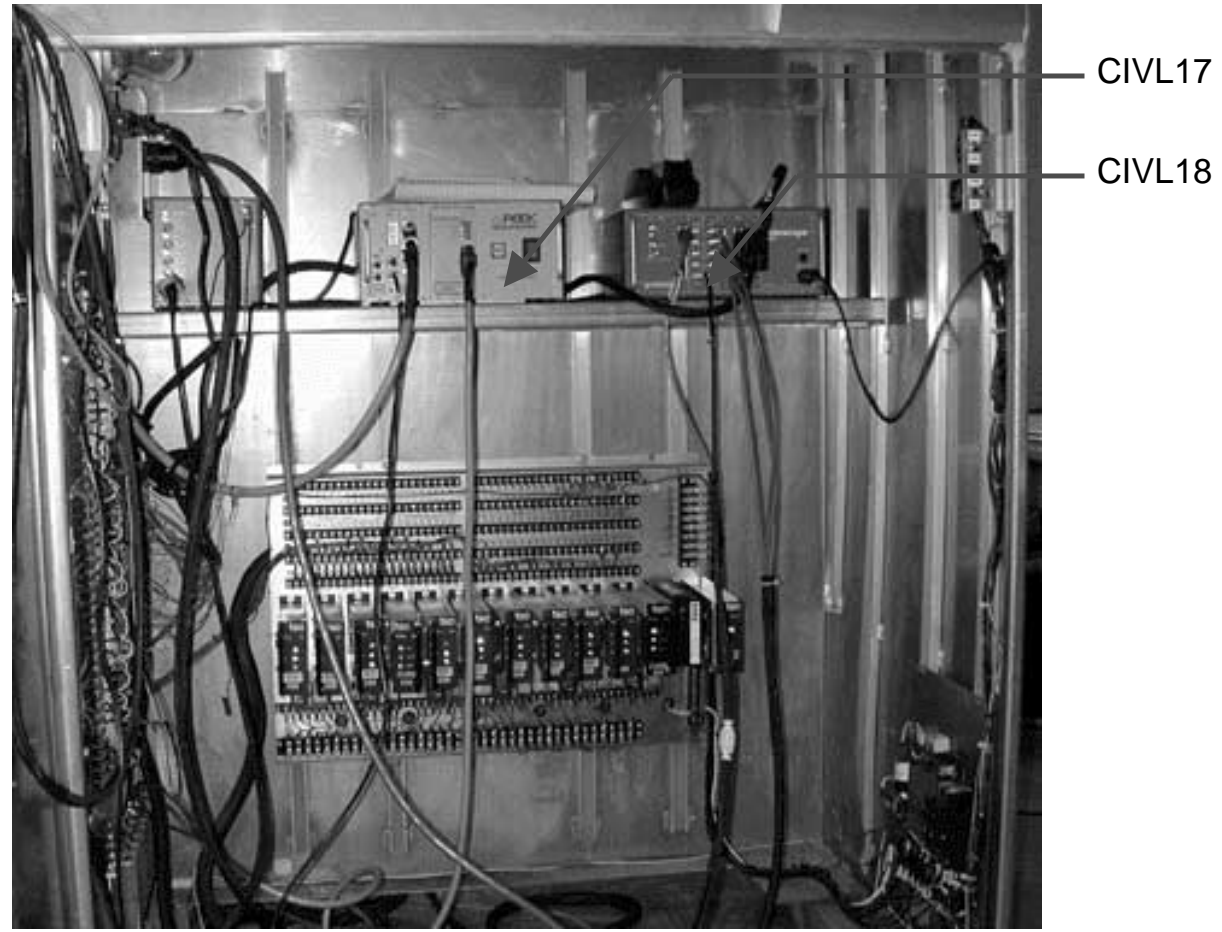

a) Cabinet and Video Detectors

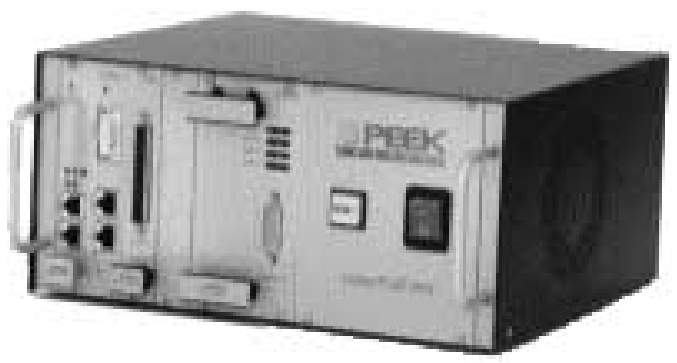

b) Peek VideoTrak-905 Close-up (CIVL17)

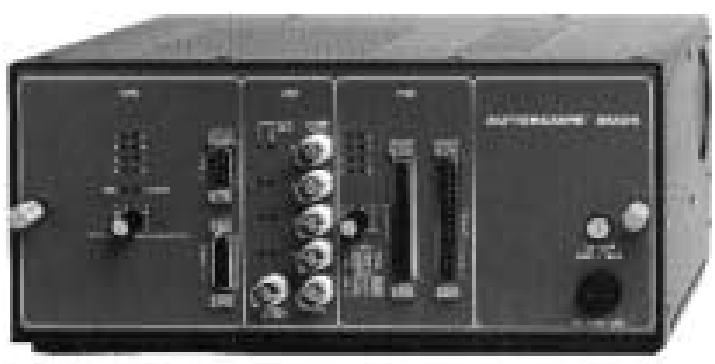

c) Econolite Autoscope Close-up (CIVL18)

Figure 3-15: Video Traffic Detector Cabinet Inside the Lab 
The rack shown in Figure 3-16 houses the CID interface between the video detection systems and the second rack. The signals from the video detection systems are interfaced using the same optically isolated modules as the INDOT cabinet interface (Figure 3-12), thus the signals from the video loops can be compared to the corresponding inductive loop signals.

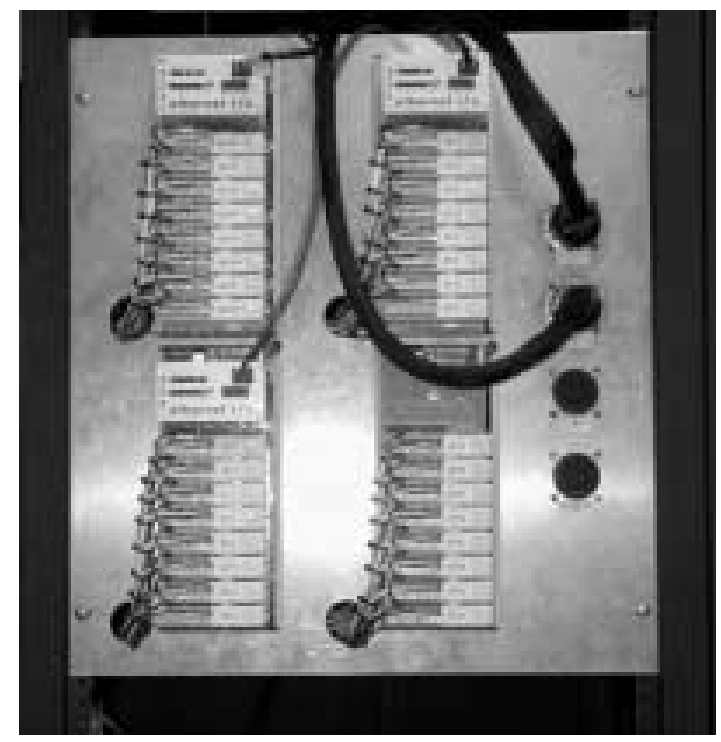

Figure 3-16: Opto22 I/O Modules - Video Detector Status Interpretation (CIVL $21-$ CIVL 23)

The second rack, shown in Figure 3-14, houses the video and CID interface. The aluminum panel on the left side of this rack is an exact replication of the intersection (CIVL16). All eight vehicle phases and the four pedestrian phases are shown at the top of the left side with small green, yellow and red lights. These lights change in real-time as the indications do at the intersection. Below the phase indications are many other small red lights, which represent the inductive loops at the intersection. When a call is placed on an inductive loop at the intersection, the corresponding red light lights up in the lab, also in real-time.

The left side of this rack also houses the CID interface in the lab for the phase indications and the inductive loop calls (CIVL15). The difference between 
this CID interface and the one at the intersection is that the one inside the lab interprets the signals from the one at the intersection. The controller (CIVL14) is also on the same panel as the CIDs inside. The controller is what causes the entire lab and intersection equipment to be coordinated, so it will be expanded upon in more depth separately.

The right side of the second rack houses the fiber video receivers (CIVL03 - CIVL07). These decode the video signal coming into the lab over the fiber optic cables, and convert them into a signal that can go to the Video Distributor (CIVL09). From here, the video is amplified and distributed to the video detection devices (CIVL17 and CIVL18), the monitors (CIVL29), and the computers (CIVL24 - CIVL27). A text overlay which shows the signal states and the detector events is placed over the video by CIVL10 - CIVL13, and then sent to one of four CPUs housed by the rack to have it encoded as a RealVideo (CIVL24 - CIVL27). A video multiplexor (CIVL28) receives the video with text overlay and allows the video to be shown one camera at a time or all four cameras at a time on the video monitor (CIVL 29). This video can also be recorded using the time lapse VCR (CIVL30).

The LCM4 controller (Figure 3-14, CIVL14) is the coordinator of all the equipment that has been assembled. In order to program it, OptoControl software is used. OptoControl uses its own high-end flow chart programming language. Once the program is finished, it is downloaded to the controller. The program will be explained succinctly here.

Every 0.05 seconds, the controller polls the CIDs at the intersection to find the states of the phase indications and of the inductive loops. It also checks the states of the video loops from the video detector CIDs in the lab. The phase indications and detector states are then placed into the text overlay on the video monitor in the lab. These processes are done on a continual basis. 
This information can also be downloaded into a database - at present the Microsoft Access Database format is being used - whenever the user specifies that they would like for data to be collected. This is not done on a continual basis, but only at certain times for research purposes.

Figure 3-17 shows the relation of the text overlay to the detection fields. Figure 3-18, Figure 3-19, Figure 3-20, and Figure 3-21 show displays from the four fixed base and focal length cameras used at the intersection. Each one is focused on one of the four approaches. These figures also show the field layouts for each of the four approaches. The screens shown in these figures are used for documenting the states of the controller as well as the "count detectors" connected to the front loops.

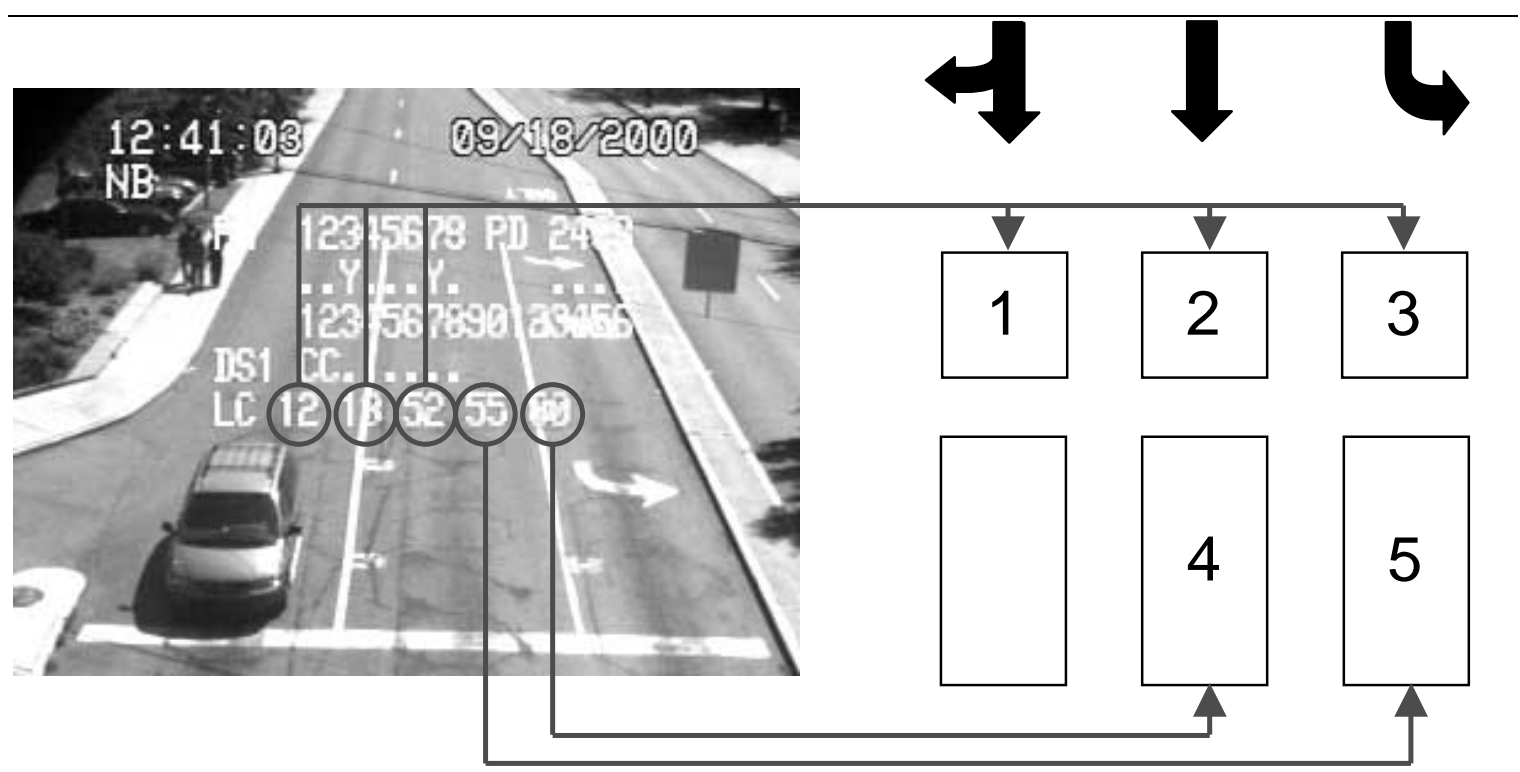

Figure 3-17: Relation of text overlay to detection fields 


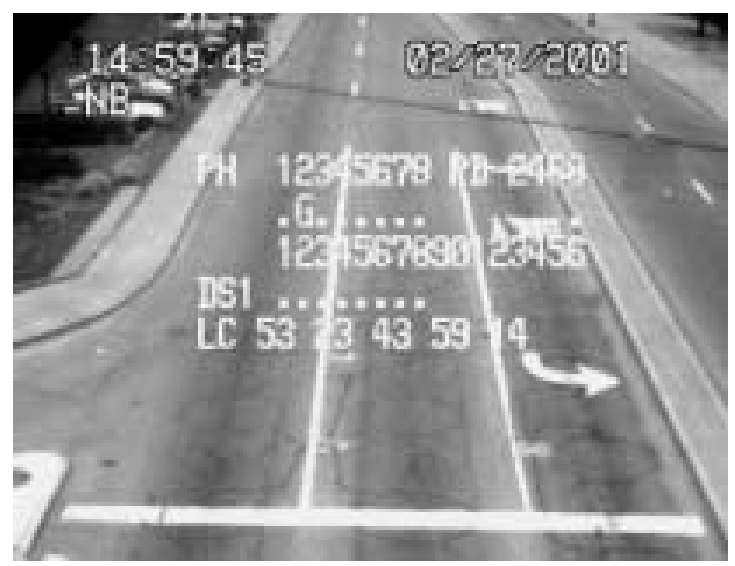

a) Northbound fields 1-5
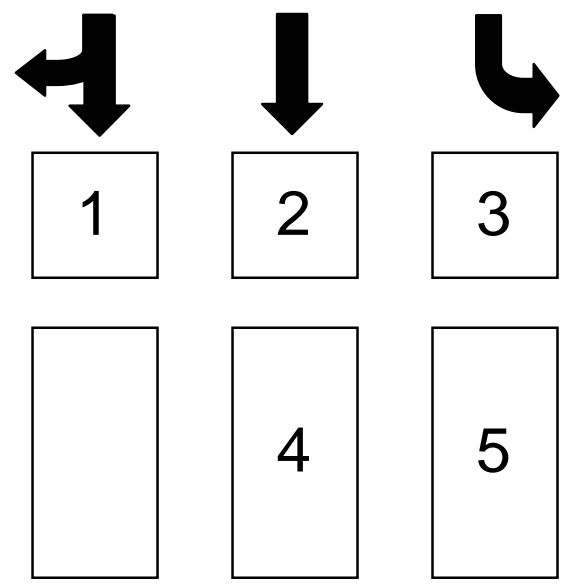

b) Northbound field locations

Figure 3-18: Northbound Fields

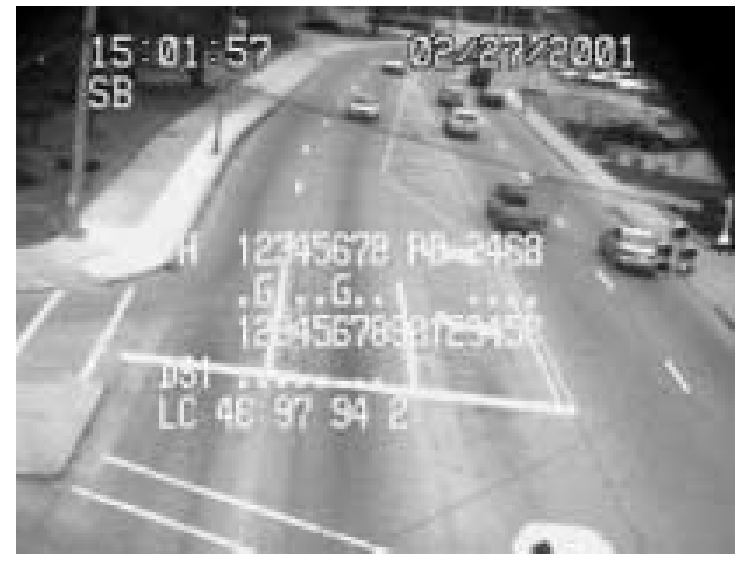

a) Southbound fields 1-4
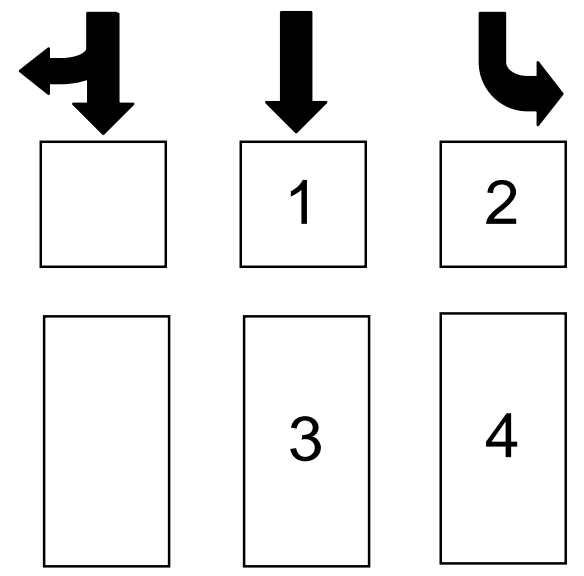

b) Southbound field locations

Figure 3-19: Southbound Fields 


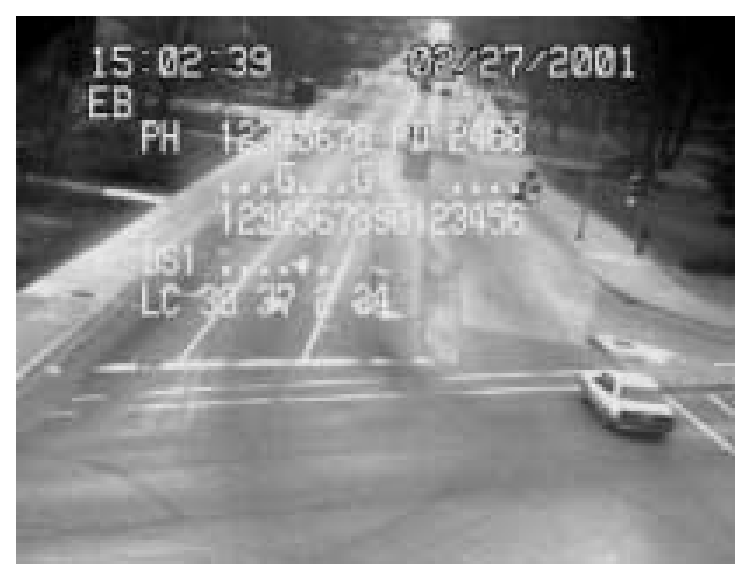

a) Eastbound fields 1-4
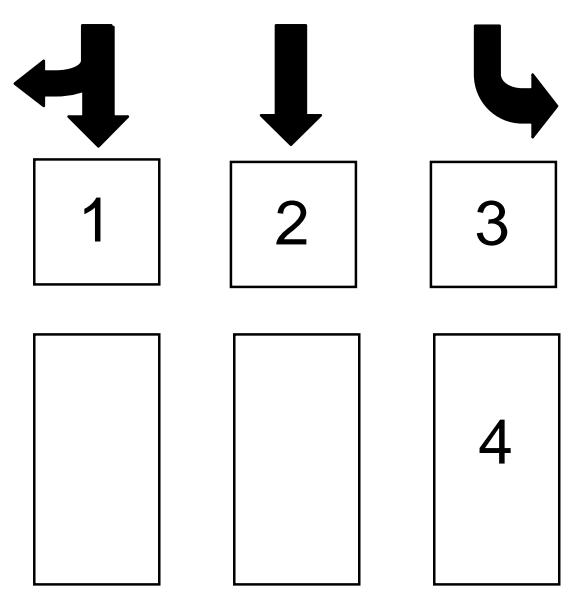

b) Eastbound field locations

Figure 3-20: Eastbound Fields

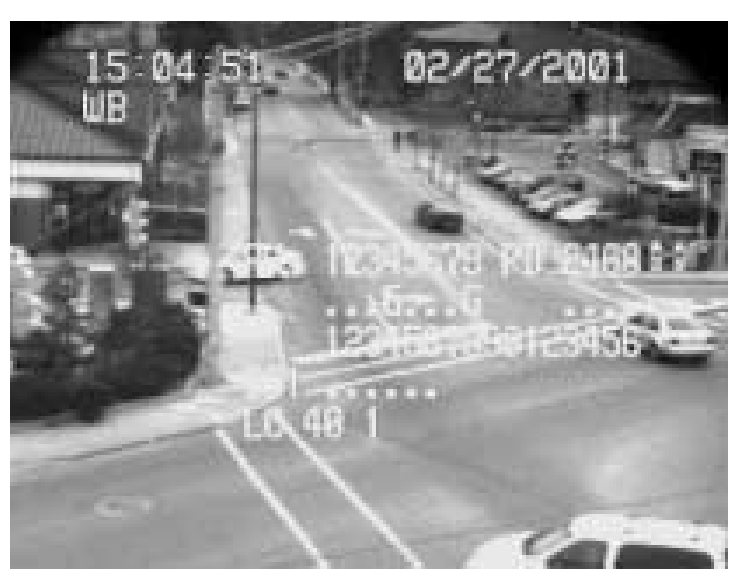

a) Westbound fields 1-4
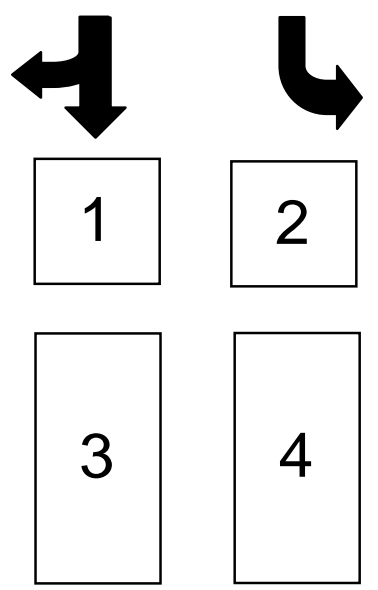

b) Westbound field locations

Figure 3-21: Westbound Fields

Figure 3-22 shows a sample video screen capture from a night-time video with the status display used for evaluating video detection units. The text overlay in this figure shows the information that is provided on the video screen. The text overlay shows which phases are being served - both pedestrian and vehicular. It also shows the detector numbers, the number of vehicles passing over each 
detector, and whether or not there is a vehicle call on the inductive loop detectors or the video detectors. For example, in Figure 3-22, one can see that phases two and five are being served. Also, one can tell that a vehicle has been detected by the inductive loops (L), and both video detectors (V1 and V2) on detector number 02.

When examining Figure 3-23, one can see which detector numbers on the text overlay correspond to which detection zones on the lanes. There are two lanes that have a detection zone number of 02 . The inductive loops in these two lanes have been wired together with an OR expression, because they serve the same traffic movements. The detection zone numbers shown here have been applied to both the inductive loops and the video detectors.

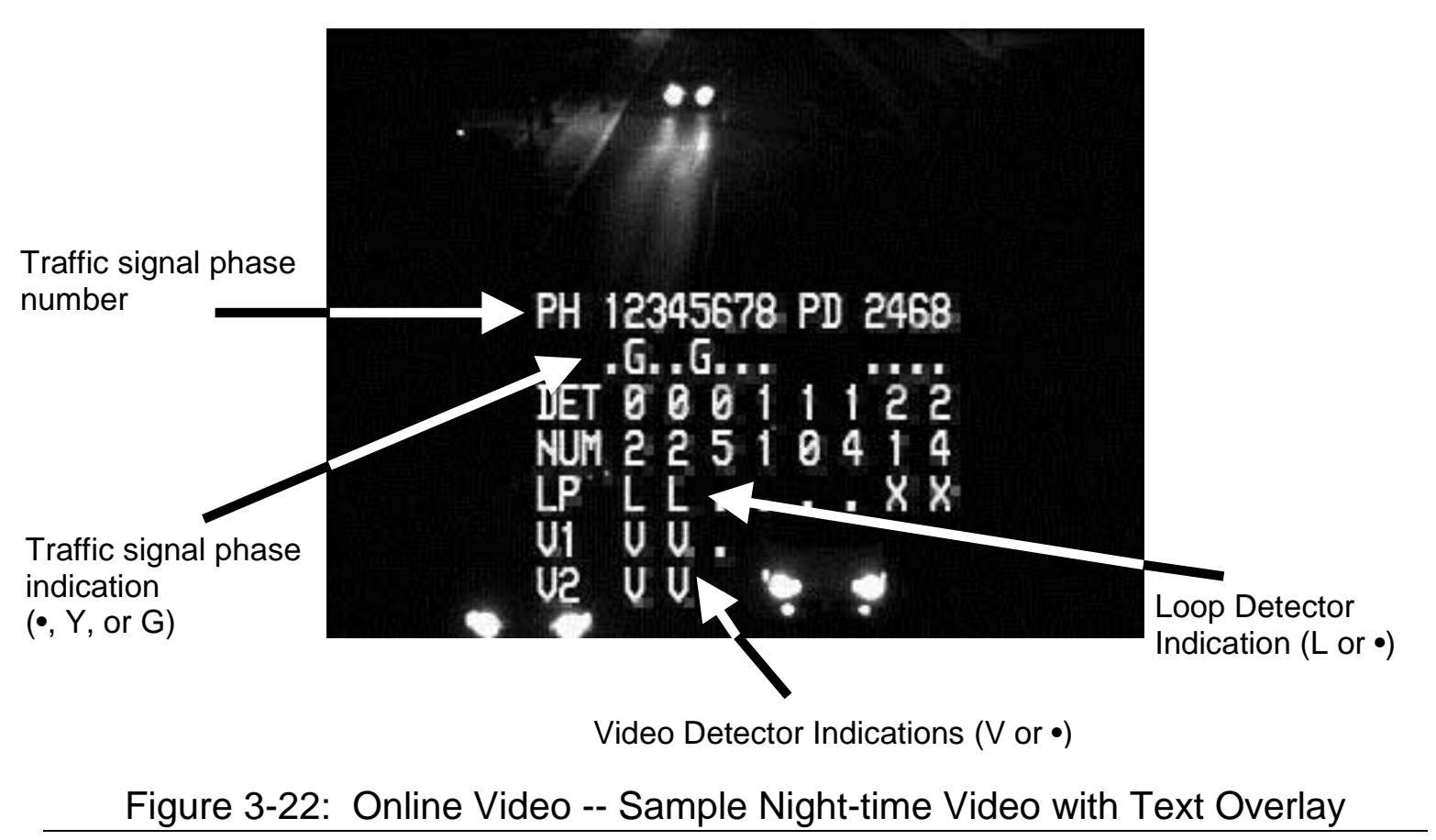

Figure 3-24, Figure 3-25, Figure 3-26, and Figure 3-27 show the northbound, southbound, eastbound and westbound approaches and their detector layouts, respectively. 


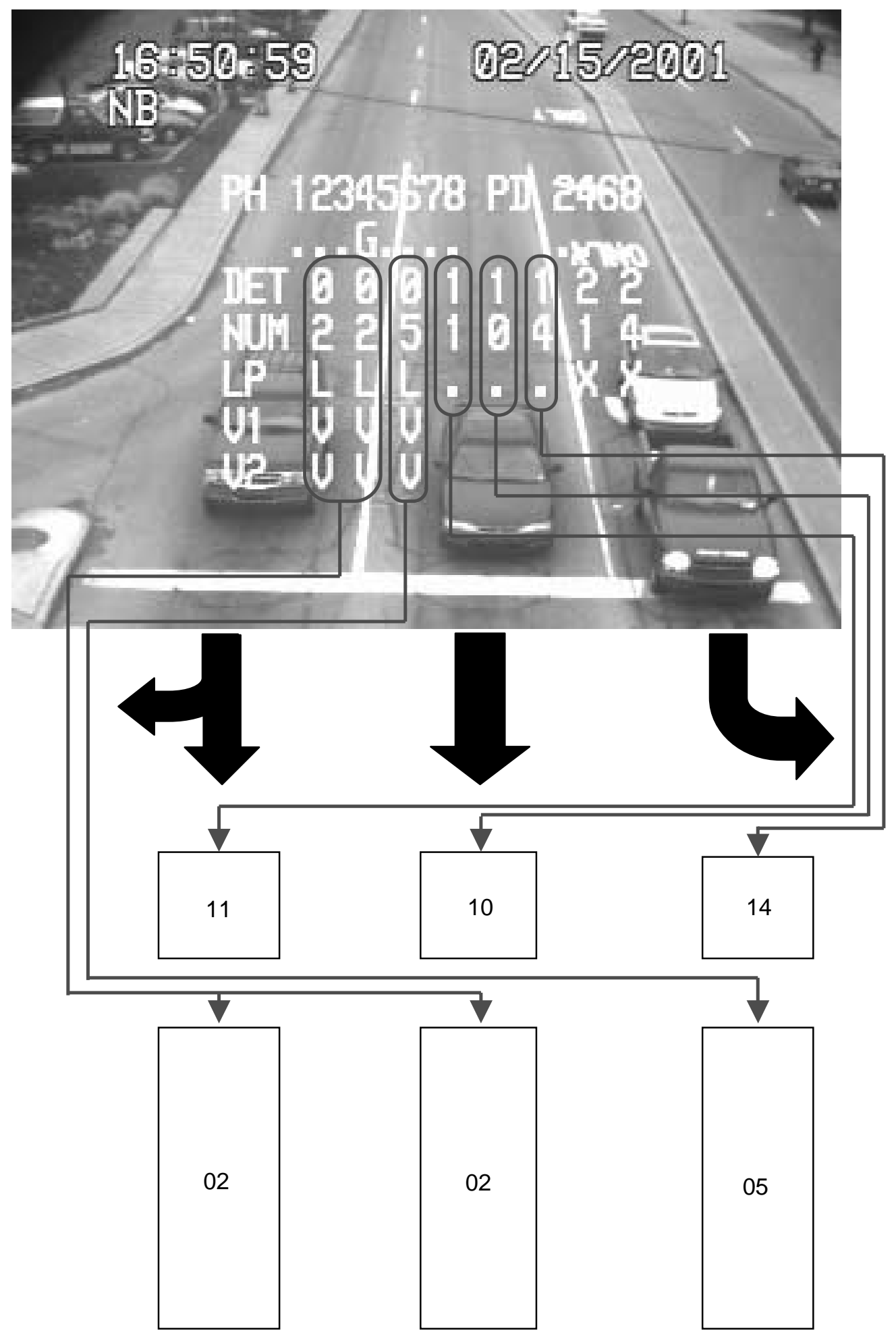

Figure 3-23: Relation of Text Overlay to Detector Layout 


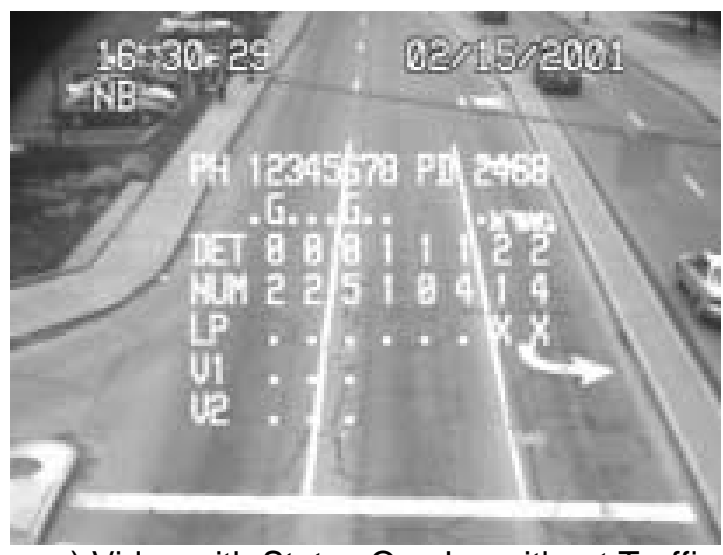

a) Video with Status Overlay without Traffic
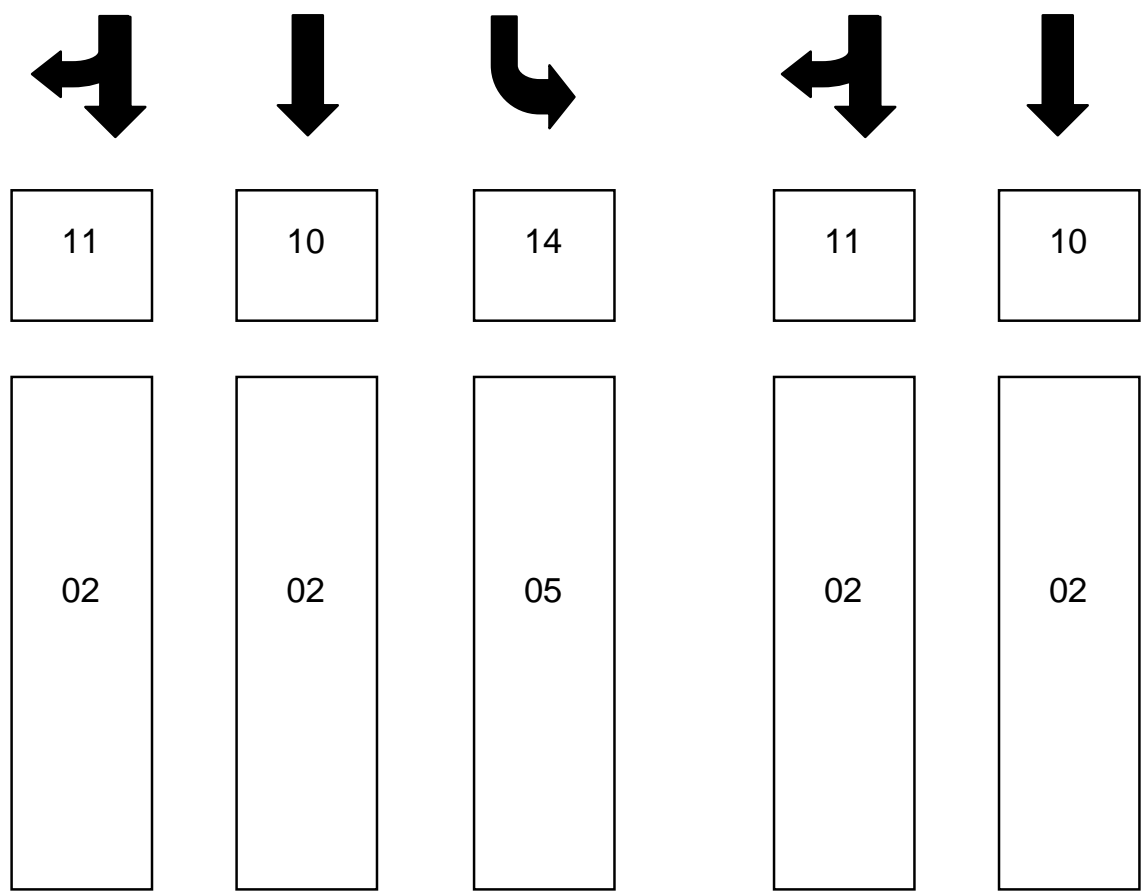

c) Detector Layout
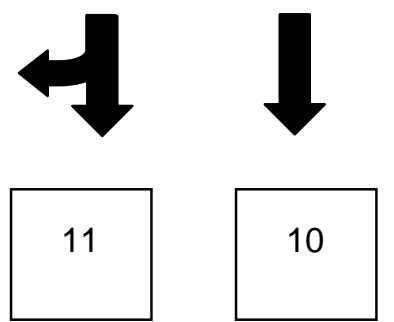

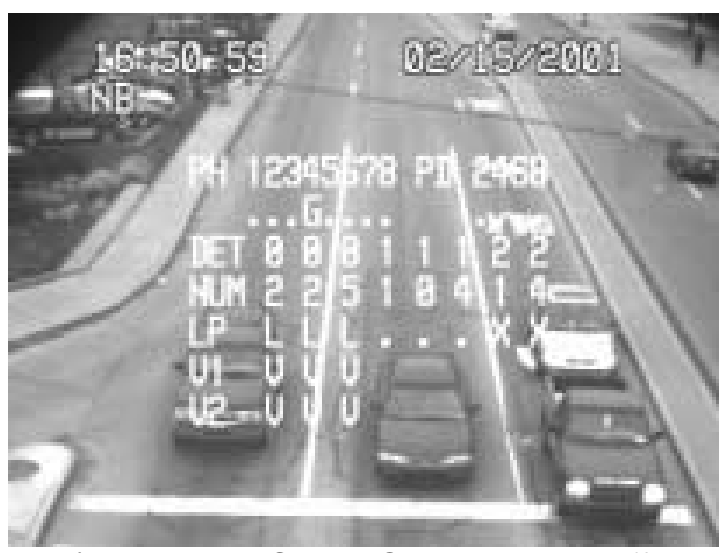

b) Video with Status Overlay with Traffic
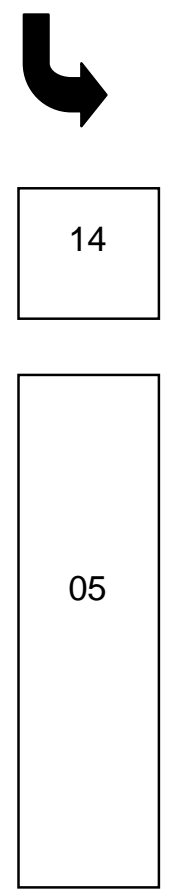

Figure 3-24: Northbound Detector Layout 


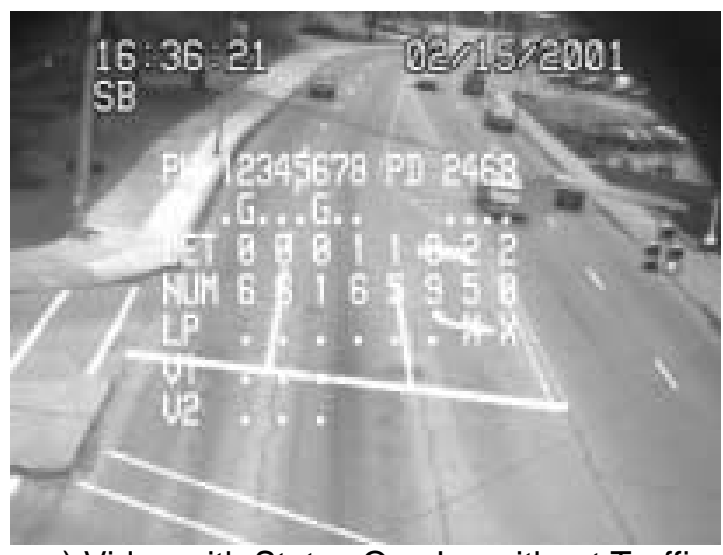

a) Video with Status Overlay without Traffic
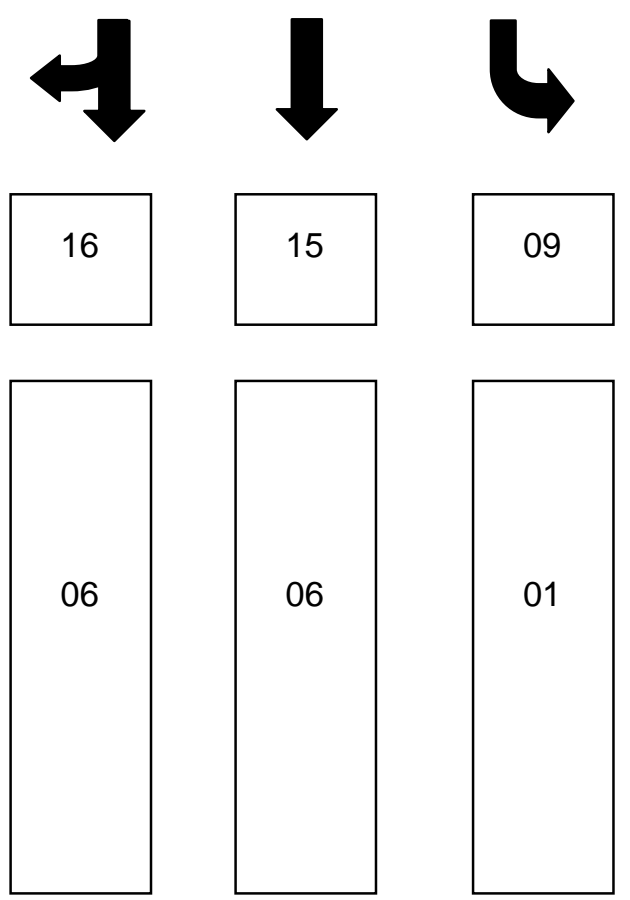

c) Detector Layout
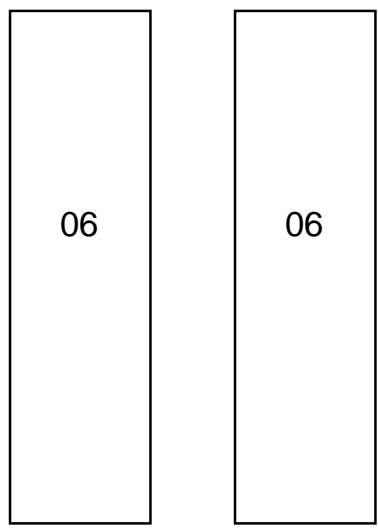

\section{$16: 37: 10$ \\ $\mathrm{SB}$}

b) Video with Status Overlay with Traffic
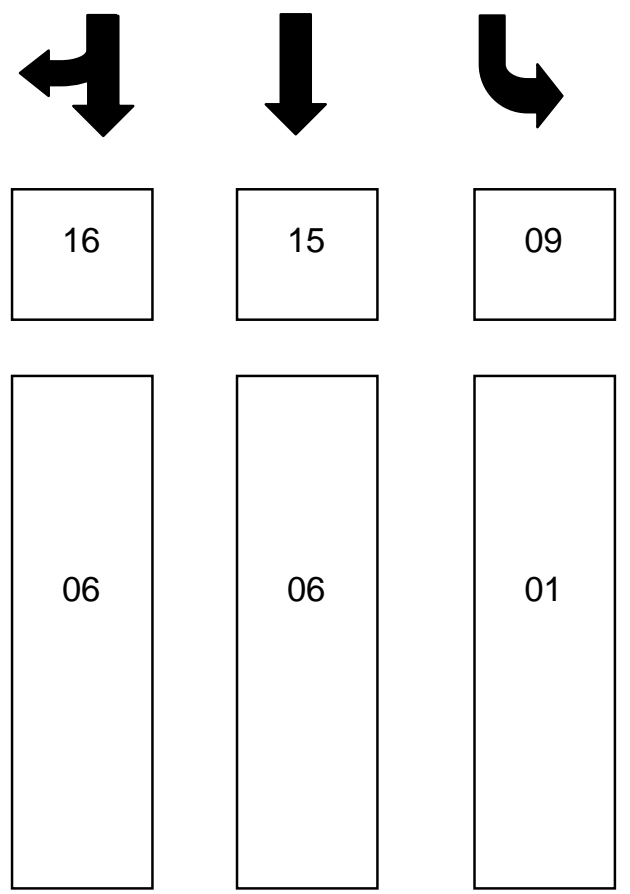

Figure 3-25: Southbound Detector Layout 


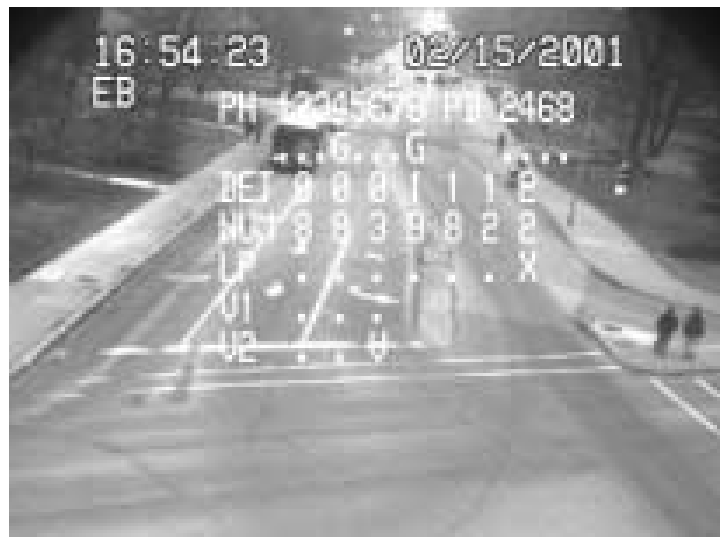

a) Video with Status Overlay without Traffic
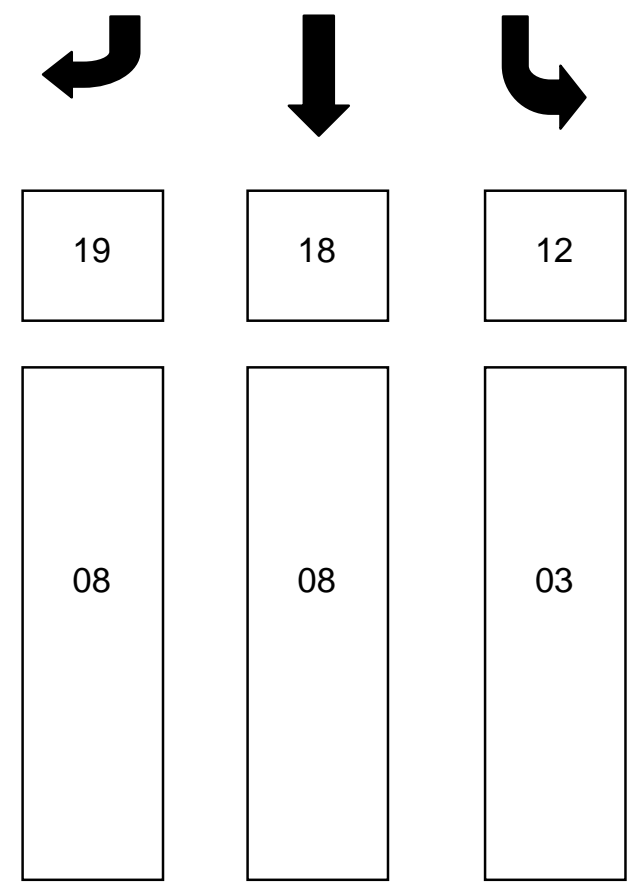

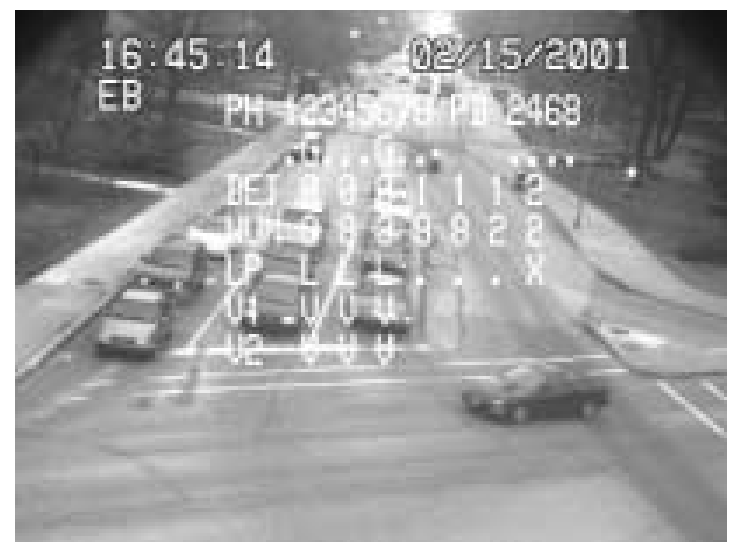

b) Video with Status Overlay with Traffic
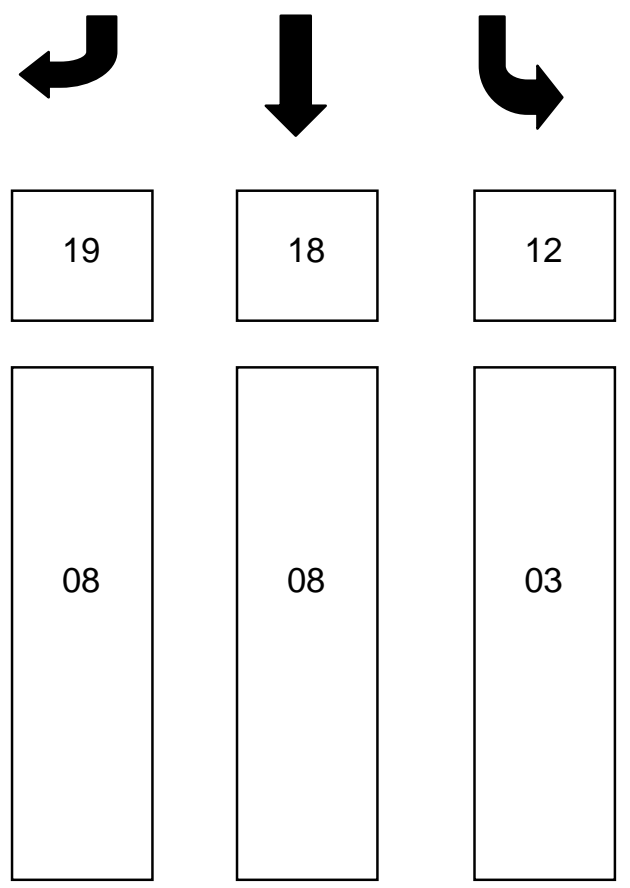

c) Detector Layout

Figure 3-26: Eastbound Detector Layout 


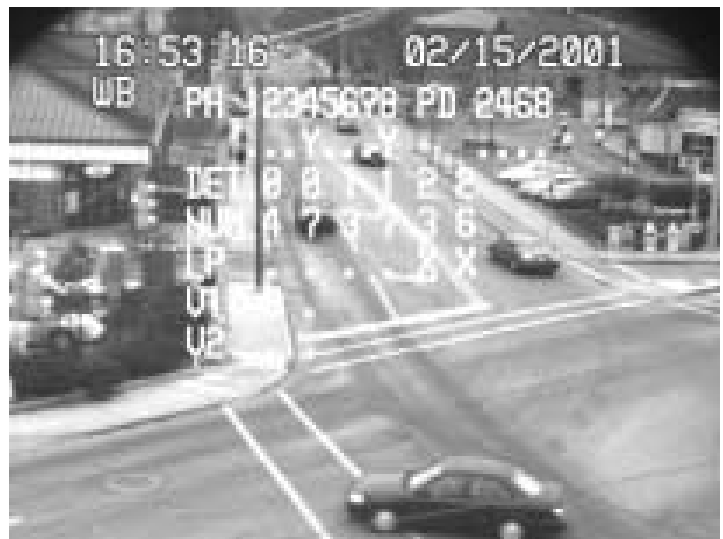

a) Video with Status Overlay without Traffic

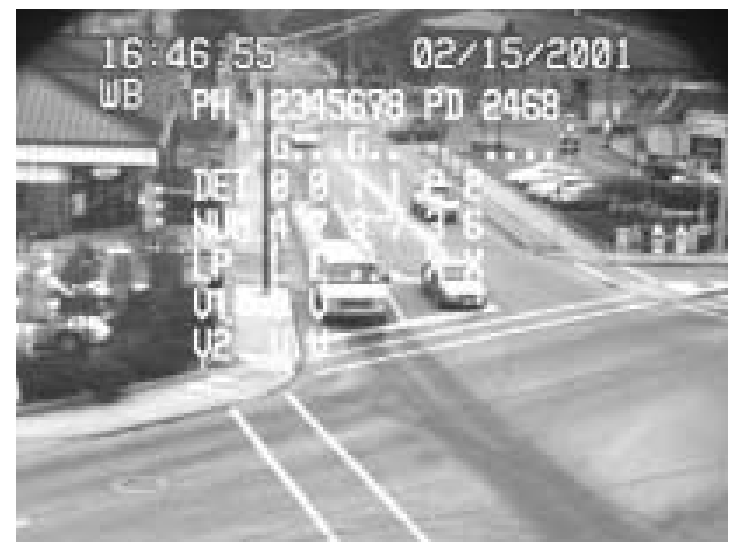

b) Video with Status Overlay with Traffic
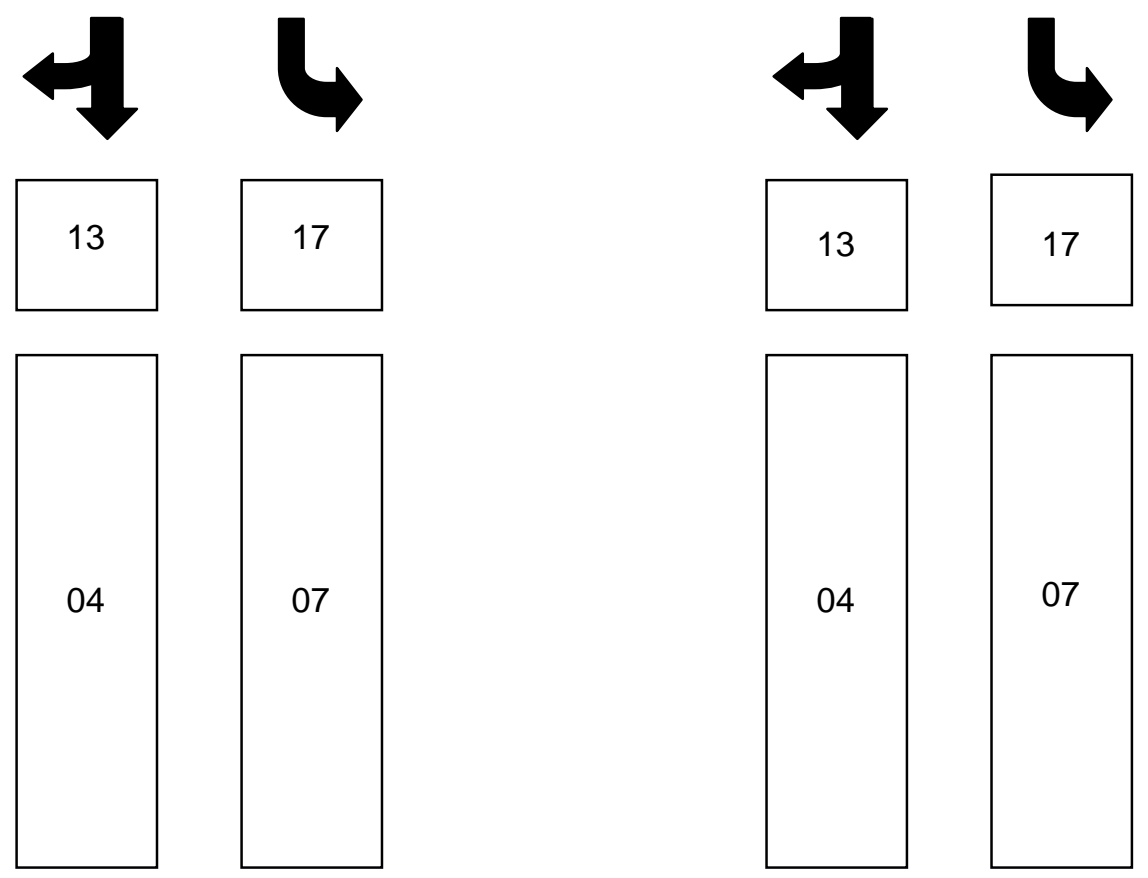

c) Detector Layout

Figure 3-27: Westbound Detector Layout 


\section{$\underline{\text { Concluding Remarks }}$}

This concludes the hardware configuration that was used for this research. In order to be able to evaluate video detection systems, it is essential that there be some type of a controlled environment that one can use to watch the performance of video detection in different conditions (e.g. weather). This environment has been created with the test-bed herein described.

The equipment that has been explained in this chapter of the report will not be used to decide whether or not video detection technologies should be employed, or even which video detection system should be used. Instead, it is used as a tool for collecting quantitative performance data that can be used by INDOT to determine if the equipment meets their needs.

The equipment has been assembled in such a way as to facilitate easy incorporation of any type of video detection equipment. Therefore, it will also allow for testing of additional video detection systems, should it be decided that they would like to test additional systems. 


\section{CHAPTER 4 - CONCEPTS OF VIDEO EVALUATION}

The hardware previously presented can be used to evaluate selected video detection systems by comparing them to inductive loop detectors in the pavement. In order to do this, the video detector layouts were initially drawn directly over the inductive loops. Because the data from the inductive loop detectors and the video detectors are collected at the same time, and in the same format, comparing outcomes from the two different detector types is relatively simple. The complication comes from the interpretation of the results. The purpose of this chapter of the report is to acquaint the reader with the evaluation measures of effectiveness (MOE) that have been used for this research.

In this research, both the terms 'error' and 'discrepancy' are used. In order to avoid any confusion between the two, the next section defines both of these terms, and relates how they are used. The following two sections are devoted to the two types of discrepancies that are possible between inductive loop detector output and Video detector output. The last three sections give a definition of the three types of likelihoods that are used as MOEs.

The first of the last three sections gives the definition of the likelihood of discrepancy between inductive loop detectors and video detectors. Secondly, a discussion of the likelihood of inductive loop error is made. Lastly, the likelihood of video detector error is addressed. 


\section{Error versus Discrepancy}

The best way to determine the effectiveness of a certain vehicle detector is to determine the amount of time that the detector produces an incorrect output. Error is an absolute term, meaning that the results have been compared to the actual, or ground truth. For part of this research, video detection is being compared to inductive loops, which also experience errors. Because this comparison is between two different types of vehicle detectors, the term that will be used is discrepancy. This term is not an absolute term, but is instead a relative term. Times when there are large discrepancies between the inductive loops and the video detectors give an indication that there may be a problem with the video detector. The recorded video with text overlay allows the user to determine whether the problem was with the inductive loops or the video detectors.

When comparing the presence output of the inductive loop detectors and the video detectors, there are four different statuses. These are documented in Table 4-1. In this table, $L$ stands for inductive loop detector, while $V$ stands for video detector. The 0 and the 1 are Boolean operators for 'does not indicate presence' and 'indicates presence', respectively. Of the four different statuses, two of them are discrepancies. These discrepancies are explained in the following sections of this chapter.

Table 4-1: Comparison Between Inductive Loop and Video Detectors

\begin{tabular}{|c|l|c|}
\hline Status & \multicolumn{1}{|c|}{ Description } & Discrepancy \\
\hline \hline L0V1 & $\begin{array}{l}\text { Loop does not indicate presence and } \\
\text { Video indicates presence }\end{array}$ & Yes \\
\hline L1V0 & $\begin{array}{l}\text { Loop indicates presence and } \\
\text { Video does not indicate presence }\end{array}$ & Yes \\
\hline L0V0 & $\begin{array}{l}\text { Loop does not indicate presence and } \\
\text { Video does not indicate presence }\end{array}$ & No \\
\hline L1V1 & $\begin{array}{l}\text { Loop indicates presence and } \\
\text { Video indicates presence }\end{array}$ & No \\
\hline
\end{tabular}


To see a graphical interpretation of the discrepancy types, see Figure 4-1. In this figure, there are four different lines, one for each of the following: Loop, Video, L0V1 Error, and L1V0 Error. When the line goes up, it means that a detector is indicating presence. When the line goes down, it means that the detector has stopped indicating presence. In this figure, the inductive loop indicates a detection of 0.8 seconds. The video detector, on the other hand, has multiple smaller detections. The two types of errors, if the inductive loop is ground truth, are shown in response to the inductive loop and video detector signals. In the actual case, the inductive loops are not perfect, and therefore the imperfection of loops will be incorporated into the discrepancy in order to estimate the likelihood of detector errors, as will be discussed in forthcoming sections of this chapter.

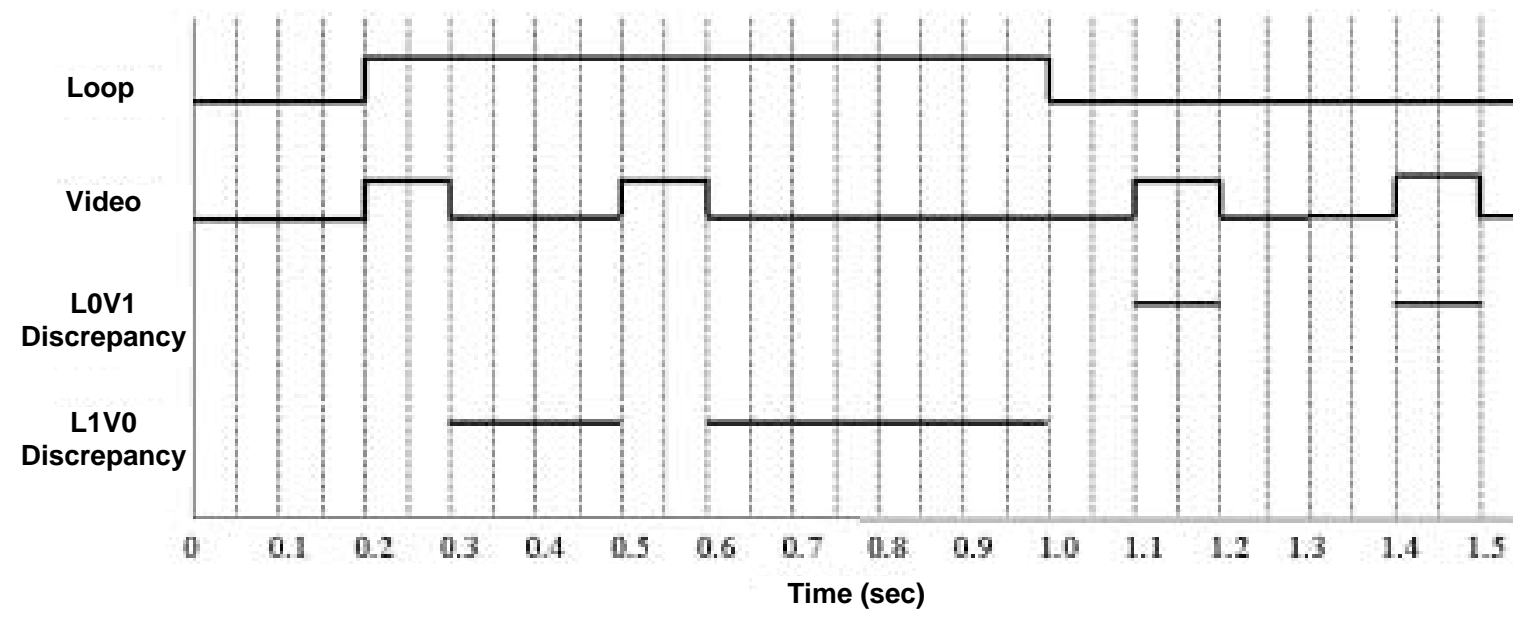

Figure 4-1: Interpretation of LOV1 and L1V0 Discrepancies

\section{LOV1 Discrepancy}

This discrepancy may be caused either by the inductive loop detector (rare cases, such as malfunctioning loop or undetected bike, motorcycle, etc.) or 
the video detector. If the inductive loop were perfect, then the LOV1 discrepancy would indicate false detections produced by the video detectors.

LOV1 errors cause a controller to operate inefficiently. They cause false calls on approaches that may not need to be served. When the false call is received, an empty approach may be given green time while vehicles are waiting on other approaches. Refer to Figure 4-2, Figure 4-3, and Figure 4-4 for example screen captures of this type of discrepancy.

Figure 4-2 shows the negative effect that headlight glare can have on video detection performance. In this case, a vehicle has pulled into the through lane approach, and is awaiting the green signal. The headlights from the vehicle are shining into the area that has been defined as the video detection area for the left turn lane. For this reason, both video detectors indicate a vehicle's presence in the left turn lane, when in reality there is none.

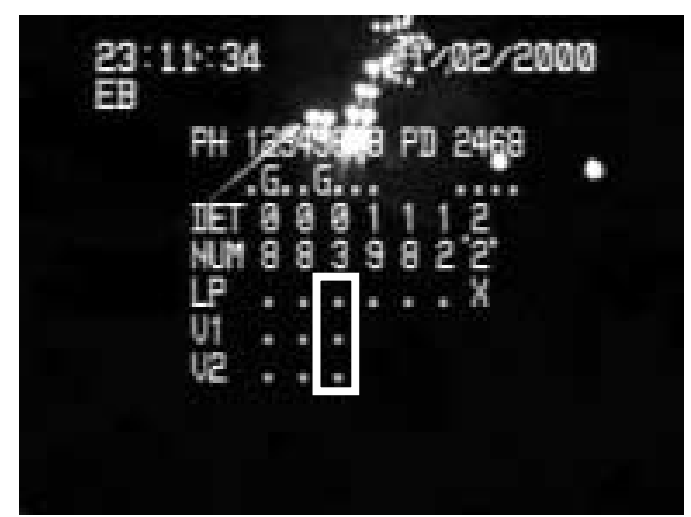

a) Before Error Begins

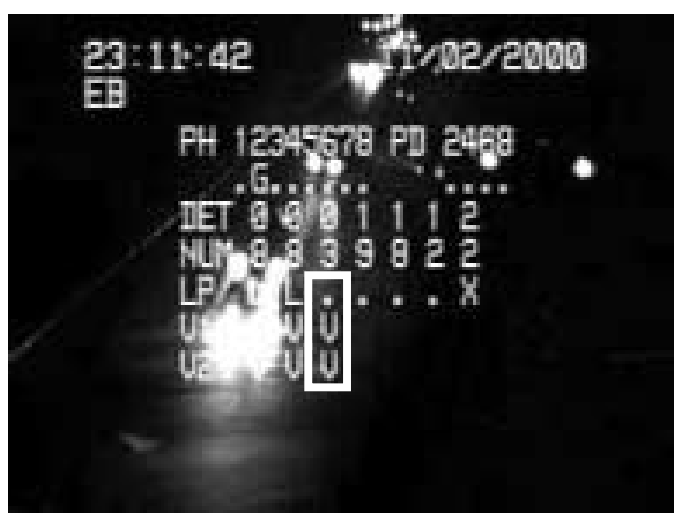

b) During Error

Figure 4-2: Example Screen Capture of LOV1 Discrepancy Caused by Headlight Glare in Left Turn Lane (Systems 1 \& 2, Before Recalibration)

Figure 4-3 shows the effect that pedestrians can have on video detection at intersections. In this figure, a pedestrian is crossing the road at the stop bar 
instead of inside the pedestrian crosswalk. This has caused both video detection systems to indicate a vehicle's presence. In areas where there is a high incidence of pedestrian crossings, and specifically, within the video detection area, this could cause a major efficiency problem for the intersection. Most video detection systems are equipped with a function that will check for directionality. By using this function, this would help to alleviate most of the pedestrian detections, since they are not walking in the same direction of the traffic flow. 


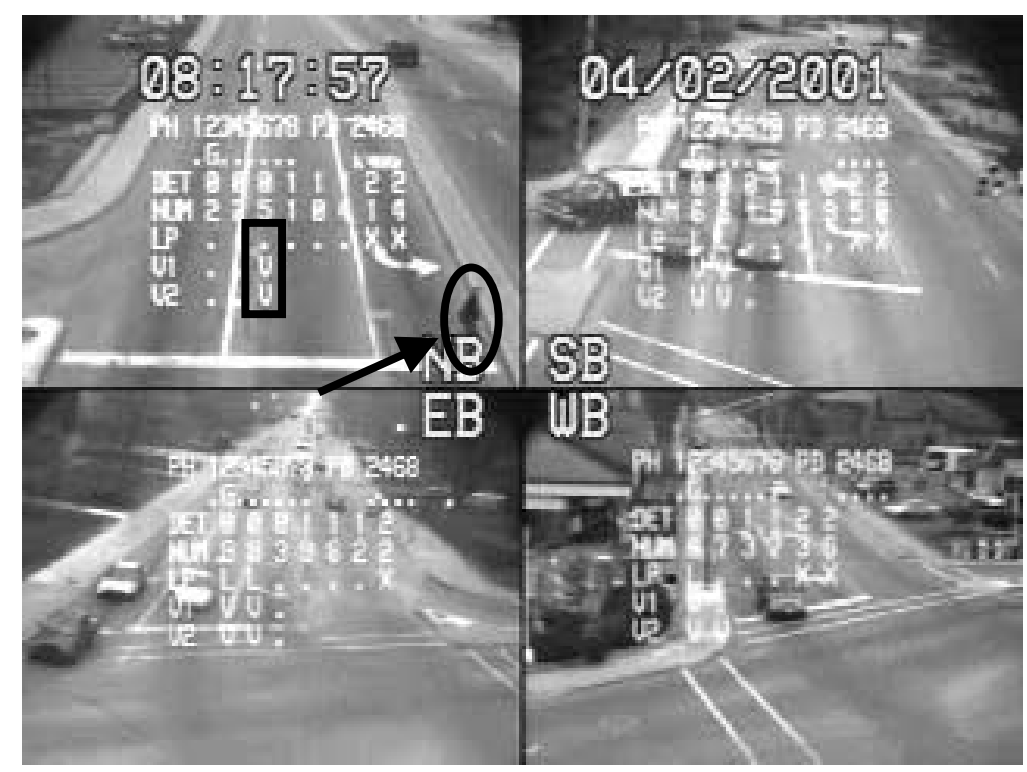

Figure 4-3: Example Screen Capture of LOV1 Error Caused by Video Detection of Pedestrian (Northbound Left Turn Lane, System 1, After Recalibration)

Figure 4-4 illustrates the negative effect that shadows can have on video detection. In this figure, the shadows from the vehicles in the left turn lane are causing Video Detection System 1 to indicate the presence of a vehicle. Again, this would cause an efficiency problem for intersections that are operating at midto-full capacity. 


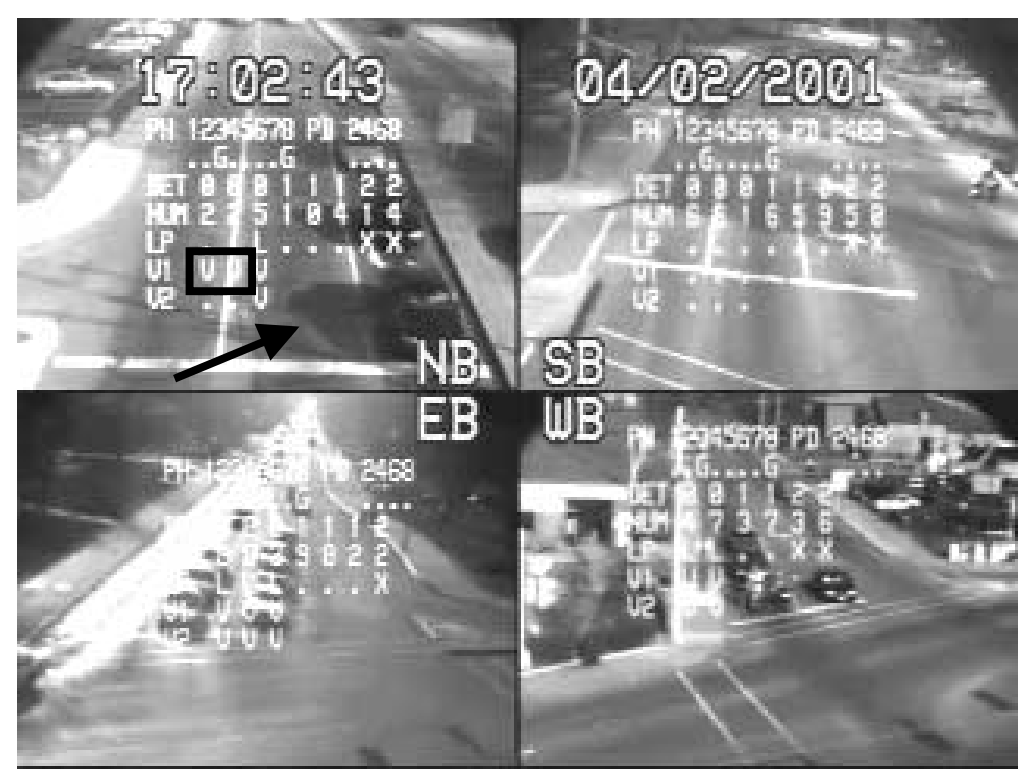

Figure 4-4: Example Screen Capture of LOV1 Error Caused by Vehicle Shadows (Northbound Through Lane, System 1, After Calibration)

\section{L1V0 Discrepancy}

This discrepancy may be caused by the inductive loop sticking on or the video detector missing or losing detections. If the inductive loop were perfect, this discrepancy would indicate the vehicle's presence missed by the video detector.

The safety implications of this discrepancy are important to note. If a vehicle is waiting at a protected left turn signal, and the video detector loses the call, then the vehicle may never be given green. This may eventually lead to motorists proceeding on a red signal. The L1V0 discrepancy could also give an indication of premature green termination, which is also a safety problem. Refer to Figure 4-5, Figure 4-6, and Figure 4-7 for illustrations of this type of error. If video detection is deployed, it is of utmost importance that the L1V0 discrepancies at the intersection are minimized. 
Figure 4-5 illustrates the fact that L1V0 errors are not always a fault of the video detectors. In this figure, a bus has pulled into the turn lane, and is awaiting the green signal. For certain reasons, perhaps too high of a sensitivity setting on the inductive loop detectors, the inductive loops in the through lanes are indicating the presence of a vehicle when, in reality, there is none present. It is through the use of the video with text overlay that these times can be ascertained.

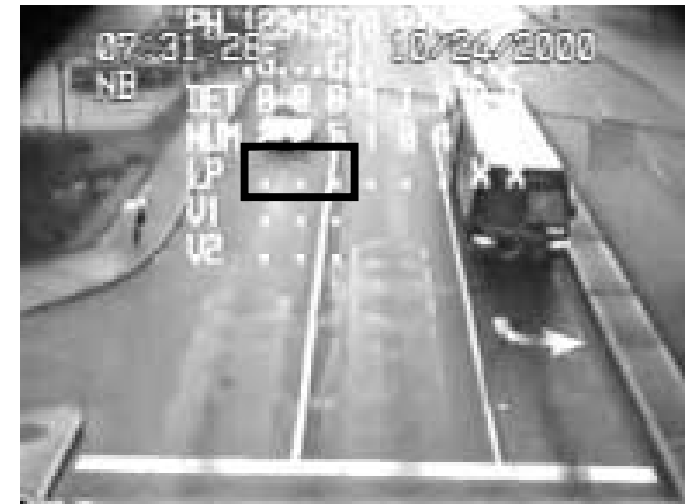

a) Before Error Begins

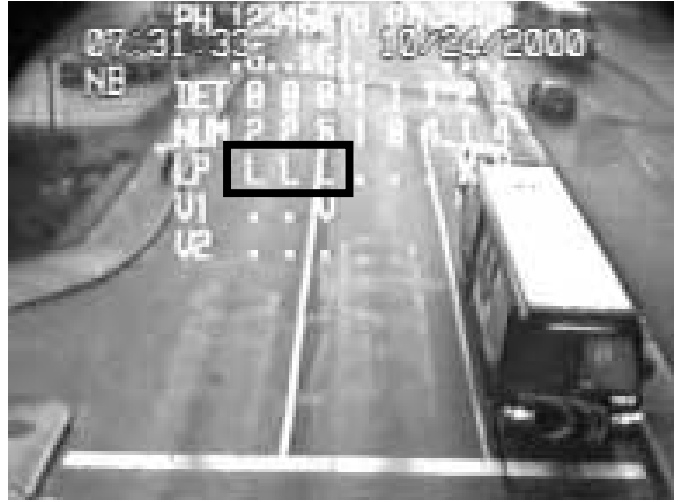

b) During Error

Figure 4-5: Example Screen Captures of L1V0 Error Caused by Inductive Loops (Before Recalibration, System 1)

Figure 4-6 shows the type of L1V0 error that is a safety hazard. In this figure, a vehicle has pulled to the stop bar, and is awaiting a green signal. Because it is night, the video detectors are detecting the headlights of the vehicles, and not the vehicle themselves. This vehicle has pulled forward enough so that the headlights are beyond the video detection zone, and thus the video detectors indicate that the vehicle is no longer present. This is not the case however, as can be seen in the screen capture, and as is indicated by the inductive loop. Extending the video detection zone somewhat past the stop bar would help to remedy this situation, but at the expense of detecting additional pedestrians. 


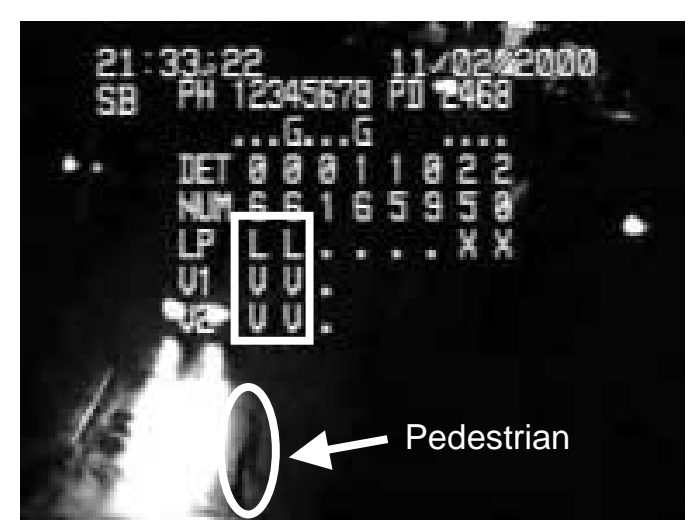

a) Before Error Begins

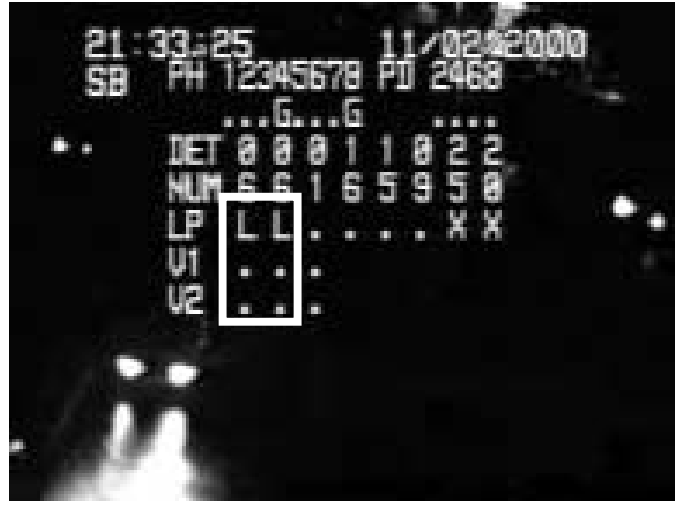

b) During Error

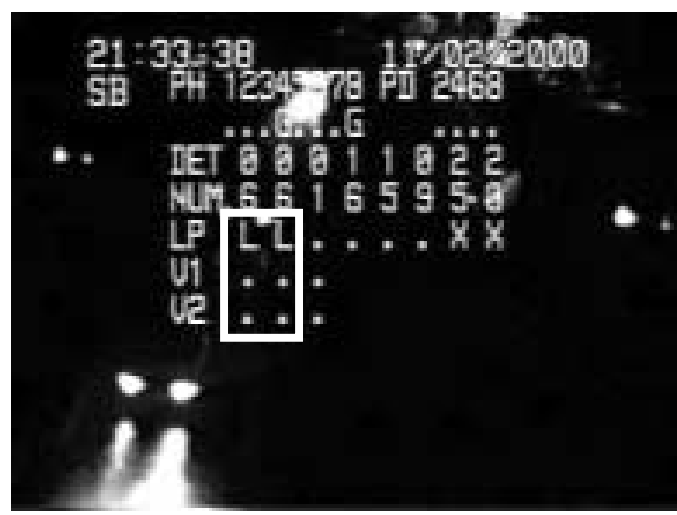

c) During Error Several Seconds Later

Figure 4-6: Example Screen Capture of L1V0 Discrepancy Caused by Poorly Calibrated Video Detector Dropping Detection at Night (Before Recalibration)

Figure 4-7, illustrates the same purpose that Figure 4-5 did. Either the inductive loop detectors in the northbound through lanes are too sensitive, and are registering the presence of vehicles in the northbound left turn lane, or they are faulty detectors. 


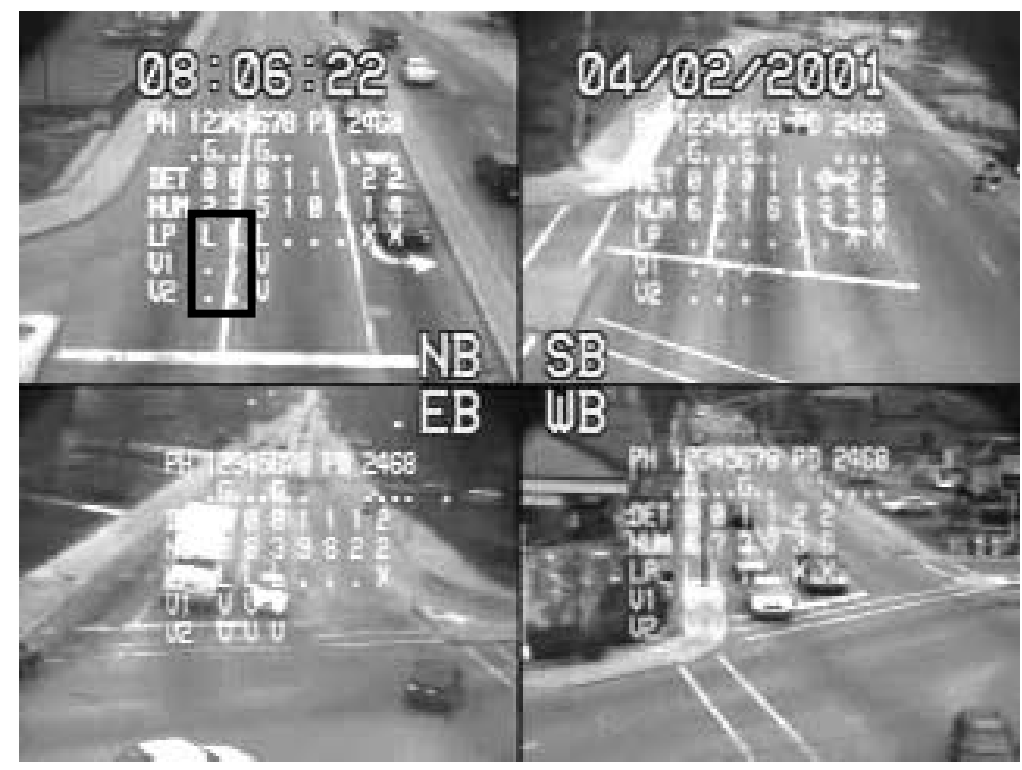

Figure 4-7: Example Screen Capture of L1V0 Error Caused by Loops (Northbound Through Lanes, After Recalibration)

\section{Likelihood of Detection Discrepancies}

The purpose of this MOE is to estimate the probability that a certain discrepancy (LOV1 or L1V0) will occur. The likelihood of detection discrepancies may be directly calculated as follows using the data collected simultaneously from the inductive loop detectors and the video detectors using the Opto Controller (shown in Figure 3-14 as CIVL14):

$$
\begin{aligned}
& \frac{D(V=0 \wedge L=1)}{D(L=1)}=P\left(V=\left.0\right|_{L=1}\right) \\
& \frac{D(V=1 \wedge L=0)}{D(L=0)}=P\left(V=\left.1\right|_{L=0}\right)
\end{aligned}
$$

where $\mathrm{D}=$ duration, $\mathrm{V}=$ video detector output, $\mathrm{L}=$ inductive loop detector output, and $P$ signifies a probability. The digits 0 and 1 stand for "does not indicate 
presence" and "indicates presence," respectively. The meaning of Equation 4-1 can be interpreted as "the probability that the video detector does not indicate the presence of a vehicle given that the inductive loop does indicate the presence of a vehicle," or "the probability of an L1V0 discrepancy taking place." Similarly, the meaning of Equation 4-2 can be interpreted as "the probability that the video detector indicates the presence of a vehicle given that the inductive loop does not indicate the presence of a vehicle," or "the probability of an LOV1 discrepancy taking place."

The L1V0 discrepancy can occur only when the loop detector indicates a vehicle's presence. Dividing the total time of L1V0 discrepancy with the total time the loop indicates vehicle presence normalizes the L1V0 discrepancy time in respect to the opportunity for this discrepancy. The LOV1 discrepancy is opposite to the L1V0 discrepancy, in that it can occur only when the loop detector does not indicate a vehicle's presence. Therefore, the total time of LOV1 discrepancy can be divided by the total time when the loop does not indicate presence in order to normalize this discrepancy with respect to the opportunity for this discrepancy.

Using Figure 4-1 as an example, the two likelihoods just discussed will be calculated here, using only 1.5 seconds as the duration of time over which the calculations will be made:

$$
\begin{aligned}
& \frac{\mathrm{D}(\mathrm{V}=0 \wedge \mathrm{L}=1)}{\mathrm{D}(\mathrm{L}=1)}=\mathrm{P}\left(\mathrm{V}=\left.0\right|_{\mathrm{L}=1}\right)=\frac{0.6}{0.8}=0.75 \\
& \frac{\mathrm{D}(\mathrm{V}=1 \wedge \mathrm{L}=0)}{\mathrm{D}(\mathrm{L}=0)}=\mathrm{P}\left(\mathrm{V}=\left.1\right|_{\mathrm{L}=0}\right)=\frac{0.4}{0.7}=0.57
\end{aligned}
$$

Typically, the value of $D$ (duration over which calculations are made) is much larger. In fact, for the purpose of this research, $D=15$ minutes. The results 
obtained for these example calculations are exaggerated in order to aid in demonstrating the meaning of each of the values.

\section{Likelihood of Detection Errors}

Another MOE helpful in measuring detection performance is the conditional likelihood of detection errors. There are two types of errors, and each has a corresponding likelihood-based MOE. One MOE tells the likelihood that a detector does not indicate the presence of a vehicle given that a vehicle is occupying the detection zone. The other one tells the likelihood that a detector indicates the presence of a vehicle given that a vehicle is not occupying the detection zone.

\section{Inductive Loop Detector Errors}

For most past detection evaluation efforts that have considered vehicle counts, there is a conventional way of measuring missed detections and the percentage of false detections, namely: missed detection rate (MDR), and false detection rate (FDR). Consider an example of detection events shown in Figure 4-8.

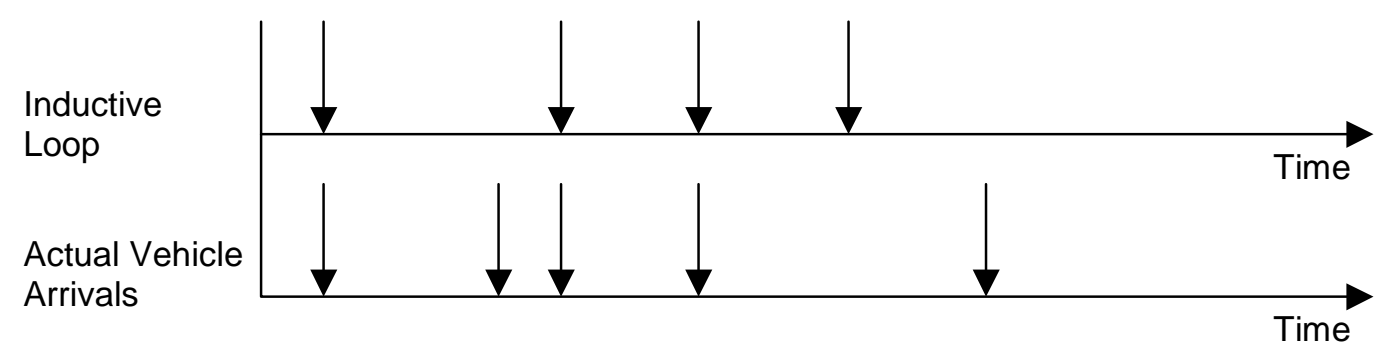

Figure 4-8: Example Detection Events

In this example, the MDR and the FDR are calculated as shown below: 


$$
\begin{aligned}
& \text { MDR }=\frac{\# \text { of actual detection events missed by loop }}{\text { total } \# \text { of actual vehicle arrivals }}=\frac{2}{5}=0.40 \\
& \mathrm{FDR}=\frac{\# \text { of false detection events reported by loop }}{\text { total } \# \text { of inductive loop events }}=\frac{1}{4}=0.25 .
\end{aligned}
$$

These calculations are for discrete events. The MDR for discrete events can be extended to continuous events (presence detectors). The likelihood of vehicle presence missed by a loop detector can be calculated in the following manner:

$$
M D R=\frac{D(L=0 \wedge T=1)}{D(T=1)}=P\left(L=\left.0\right|_{T=1}\right),
$$

where $D=$ duration, $L=$ inductive loop output, and $T=$ truth. This conditional likelihood can be interpreted as "the probability that the inductive loop does not indicate the presence of a vehicle, given that the vehicle is present."

Likewise, the FDR calculation can be extended:

$$
F D R=\frac{D(L=1 \wedge T=0)}{D(L=1)},
$$

where the symbols have the same meaning as for the likelihood of missed loop detector errors. The likelihood in Equation 4-6; however, is inconsistent with Equation 4-5, in that the denominator considers the duration of time when the loop is indicating a vehicle's presence, and does not make reference to the ground truth as the first one does. For the sake of consistency with the first likelihood, Equation 4-6 has been modified to the following: 


$$
\frac{\mathrm{D}(\mathrm{L}=1 \wedge \mathrm{T}=0)}{\mathrm{D}(\mathrm{T}=0)}=P\left(L=\left.1\right|_{\mathrm{T}=0}\right)
$$

This conditional likelihood is now consistent with Equation 4-5, and has greater meaning to this research. It may be interpreted as "the probability that the inductive loop indicates the presence of a vehicle, given that a vehicle is not present." The reader may notice that both Equations 4-5 and 4-7 have a denominator that represents the amount of time during which it is possible for a certain type of error to occur. In other words, Equation 4-7 could also be interpreted as "the duration of time in which there was an L1T0 error, divided by the duration of time during which an L1T0 error could have taken place."

\section{Video Detector Errors}

The conditional likelihoods of video detector errors are derived in exactly the same way as those for inductive loop detectors. The probability that a video detector does not indicate the presence of a vehicle given that a vehicle is present is represented by the following likelihood:

$$
\frac{\mathrm{D}(\mathrm{V}=0 \wedge \mathrm{T}=1)}{\mathrm{D}(\mathrm{T}=1)}=\mathrm{P}\left(\mathrm{V}=\left.0\right|_{\mathrm{T}=1}\right)
$$

Likewise, the probability that a video detector indicates the presence of a vehicle given that a vehicle is not present is represented by the following likelihood:

$$
\frac{\mathrm{D}(\mathrm{V}=1 \wedge \mathrm{T}=0)}{\mathrm{D}(\mathrm{T}=0)}=\mathrm{P}\left(\mathrm{V}=\left.1\right|_{\mathrm{T}=0}\right)
$$

Once these likelihoods are known, it is possible to directly compare two or more video detection systems, free from the effect of the imperfection of inductive loop detectors. 


\section{CHAPTER 5 - SOFTWARE DEVELOPMENT}

\section{Chapter Overview}

This chapter will describe the software that has been developed in conjunction with this research. The purpose for which the software was developed was to aid in data reduction and calculations. It was noted in Chapter 3 that the database type that is being used to store the data is the Microsoft Access database. The Access database uses the Microsoft Jet database engine in order to access the data in its tables. Because Microsoft Visual Basic 6.0 also provides the use of the Microsoft Jet database engine, this is the programming language of choice for this research. The amount of data that is collected during each data collection, along with the amount of calculations that need to be performed is the reason for which a more mainline spreadsheet program could not be used. For example, Microsoft Excel 2000 has a maximum number of rows in its worksheets of nearly 65,600 . Access is different in that it is only limited by the amount of resources the host computer has. The function of the software that will be discussed in this chapter is to do many of the calculations that are necessary for evaluation of the video detection systems, to produce preliminary graphs that will allow for viewing the data, and also to reduce the data to a format that can easily be imported into Excel for more robust graphs to be plotted.

\section{The Graphical User Interface}

The graphical user interface (GUI) is shown in Figure 5-1. This interface is divided up into segments that are divided by horizontal lines. Steps $1-3$ are mandatory, while steps 4 and 5 have default values if the user chooses not to input any values. Even though several of the steps are intuitive, they will be outlined in detail here. 


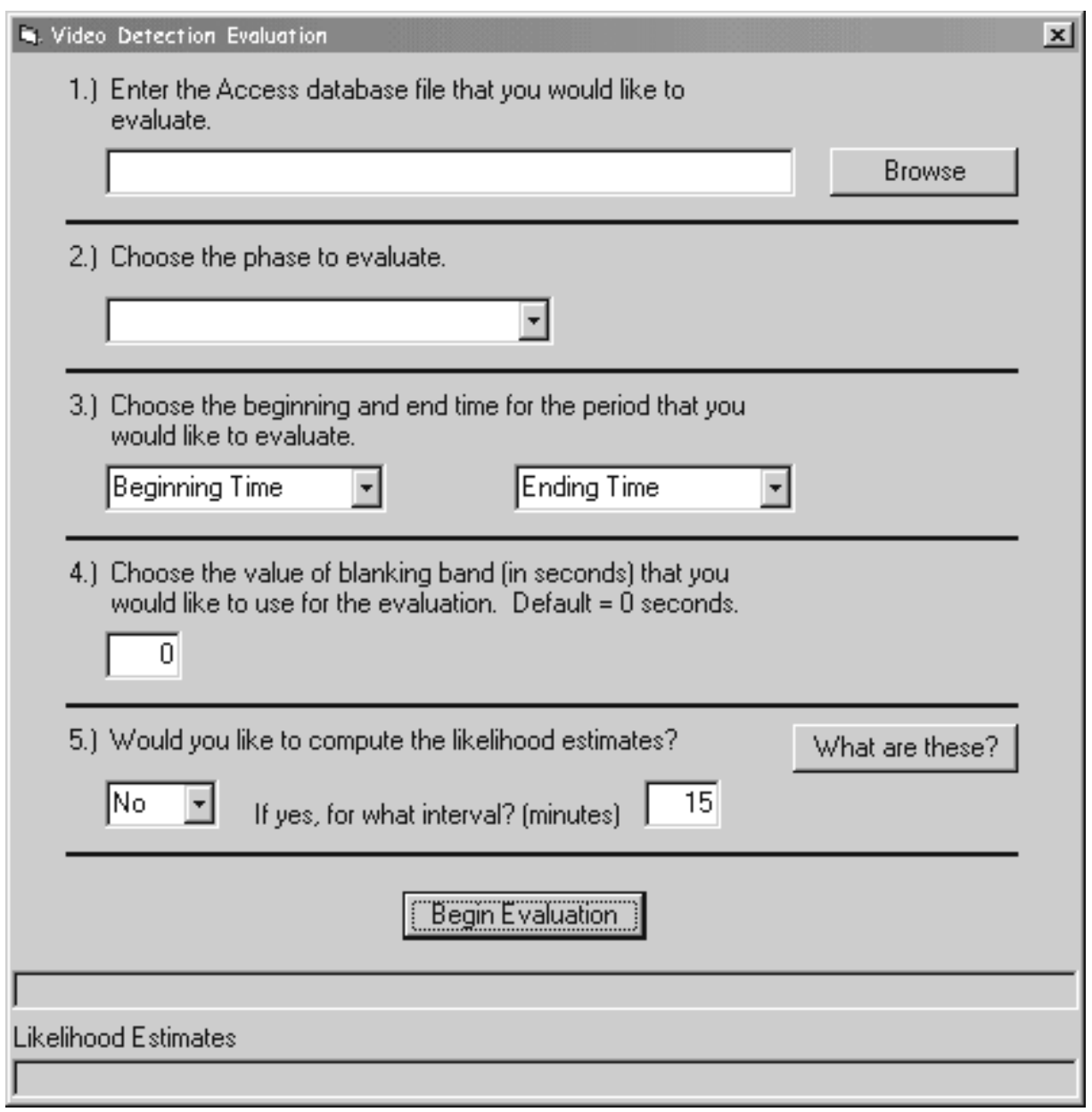

Figure 5-1: Graphical User Interface

\section{Step One}

Step one of the GUI asks for the user to insert a database file location. This database is obtained by collecting data with the hardware that has previously been described. Each database is set up in the same way, such that the program deals in much the same way with each database file. The user has been given the option to browse for the file using the popular Windows Open File dialogue box. 


\section{Step Two}

All of the data is separated by phase in the database, and so lends itself to evaluation by phase. For this reason, the software has been written in such a way that calculations are done for one phase at a time. This allows for quicker execution of all calculations, and is very helpful when the user only would like to examine the video setup on one or two approaches, and not the entire intersection. If the user does want to examine the entire intersection, they need only rerun the program for each phase.

\section{Step Three}

Step three allows the user to specify the beginning and ending of the period that they would like to analyze. For instance, if the user is not interested in the entire period of data that was stored, but only a specific portion of it, they may specify the period that they are interested in. Most of the data collections that were performed for this research were started in the afternoon of one day, and then stopped in the afternoon on the next day. Because of this, the pull down menus allow the user to select from "Day 1, Midnight" until "Day 3, Midnight." This encompasses 48 hours, and thus is a long enough time period for even the longest of data collections. As was stated previously, this step is mandatory, and must be entered in order for the software to proceed.

\section{Step Four}

Step four allows the user to place a "filter" on the calculations. In calculating the discrepancies between the inductive loops and the video detectors, the user may not be concerned about discrepancies that are below a certain duration of time. They can specify this length of time here, and when the calculations are made, those discrepancies that are below the specified duration will not be included in the evaluation. The default value for step four is zero seconds. 


\section{Step Five}

Step five allows the user to decide whether they would like the "Likelihood Estimates" to be calculated. In short, the likelihood estimates are the probabilities that the video shows no vehicles when the inductive loop shows a vehicle and the probability that the video detector shows a vehicle when the inductive loop does not. These estimates can then be compared to the ground truth data for inductive loops, thus giving ground truth data for the video detectors. These were discussed in detail in Chapter 4.

\section{Begin Evaluation}

Once steps $1-5$ have been completed, then the user will click on the "Begin Evaluation" button. Below this button are two progress bars. The top progress bar shows the progress for all calculations, except the likelihood estimates. The second progress bar shows the progress of the likelihood estimate calculations. Once all calculations have finished, the "Results" screen appears. Figure 5-2 shows the result screen, as it first appears when calculations have finished. It is blank because none of the options have yet been selected. Once the buttons on the left side of the screen are clicked on, the corresponding graphs will be displayed. This screen will be explained in more detail after the database structure has been explained. 


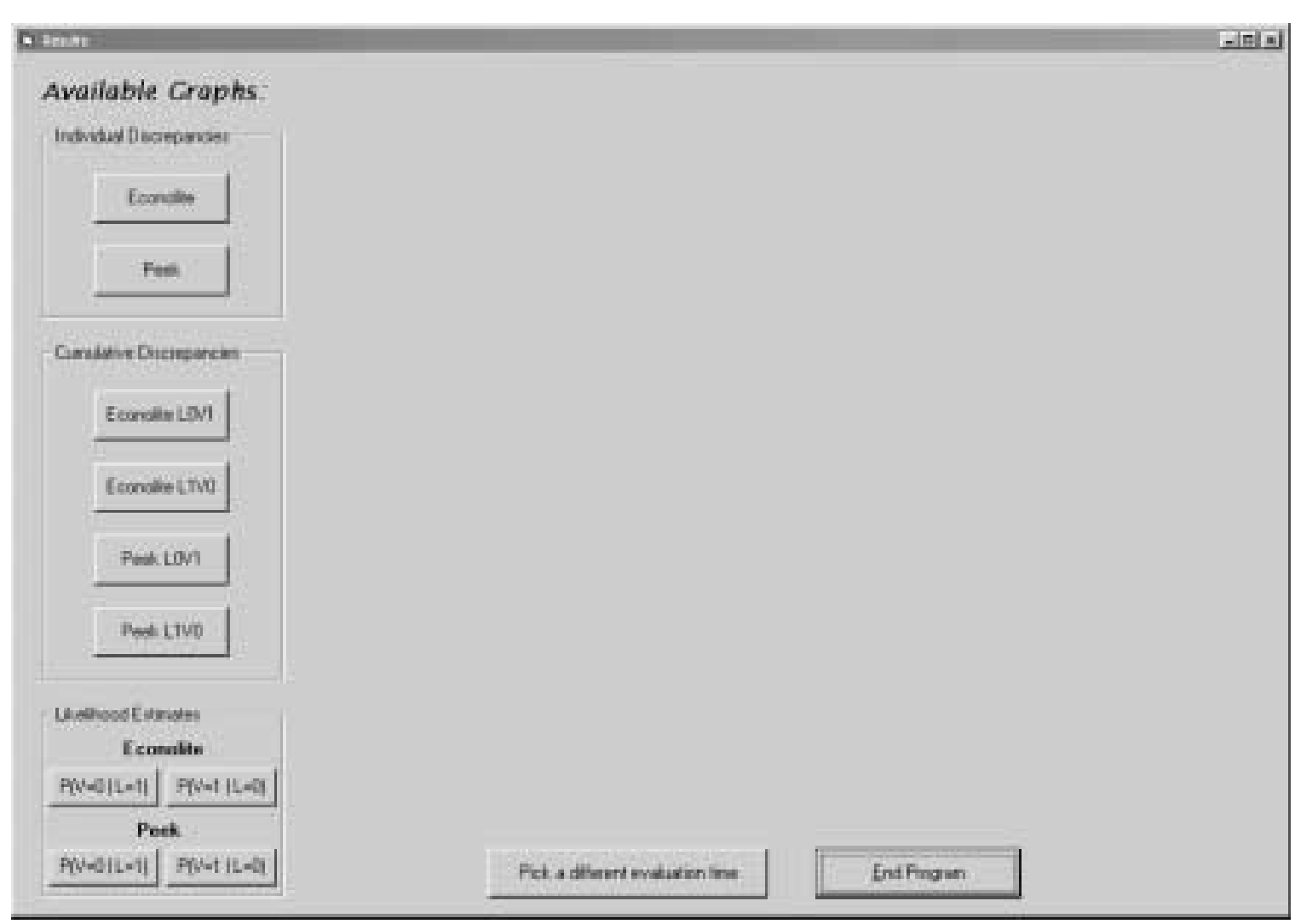

Figure 5-2: Results Screen

Also, as the program is performing the necessary calculations, several new tables are created in the Access database that hold the results of the calculations. The user may open these tables in Access, or export them to Excel if they would like to make more robust graphs than those available through the Video Detection Evaluation program.

\section{Data Structure}

The Access database that is fabricated by the Opto22 Controller (Figure 3-14) during data collection contains around 170 tables. One table gives the starting and ending times of the data collection, along with the corresponding seconds from midnight for the starting time. A sample of this table is shown in 
Figure 5-3. All times recorded in other tables in the database are in the format of seconds from midnight on the first day of collection.

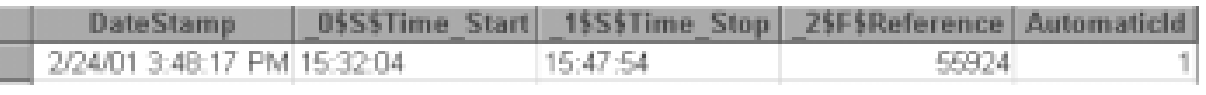

Figure 5-3: Sample Data Collection Times Table

As was previously stated, the database is arranged by phase. Therefore, each detector, whether it is an inductive loop or a video detector, is labeled by the phase that it serves. For example, the inductive loops that call phase one are labeled as LP01, and the video detectors that would call phase one are labeled EL01 $(E L=$ Econolite) and PK01 (PK = Peek). Each detector has two tables that correspond to it in the database. One of the tables give the times that the detector turned on, and the other gives the times that the detector turned off. Beside the inductive loops that call the vehicular phases, there are also other inductive loops that are being monitored, and this data is being sent to the database, as well.

Figure 5-4, Figure 5-5, and Figure 5-6 show example start and stop tables of the Autoscope, Video-Trak, and the inductive loops, respectively. With the information shown in these figures, one could calculate the discrepancy between the occupancy times of the inductive loops and the video detectors. The Opto22 Controller has been programmed such that it recognizes discrepancies between the inductive loops and the video detectors, and adds the necessary start and stop tables into the database to account for these discrepancies. This allows for fewer post-processing calculations. 


\begin{tabular}{|c|c|c|c|}
\hline DafnStamp & |reden & tait & Autumafete \\
\hline 3501 951.5SAU & 0 & 4439070 & 801 \\
\hline $350195153 \mathrm{AU}$ & 1 & 4440651 & 90 \\
\hline $3.501951 .53 \mathrm{AU}$ & 2 & 449931 & 903 \\
\hline $3501951534 \mathrm{~K}$ & 3 & 44532 If & 904 \\
\hline $3501851.53 \mathrm{AM}$ & 4 & 4541.25 & 906 \\
\hline $350195153 \mathrm{AM}$ & 5 & 4456624 & 906 \\
\hline $350195153 \mathrm{AM}$ & 6 & $44 \times 125$ & 90 \\
\hline $350195153 \mathrm{AM}$ & 7 & 4454391 & 908 \\
\hline 3501961.59 คM & 8 & 4475.59 & 909 \\
\hline $350195153 \mathrm{AU}$ & 9 & 4479316 & 910 \\
\hline $3501951.53 \mathrm{AM}$ & 10 & 447317 & G1t \\
\hline $350195153 \mathrm{AM}$ & 11 & 45069 & 912 \\
\hline $3501951.53 \mathrm{NW}$ & 12 & 25153.63 & 913 \\
\hline $3501951.53 \mathrm{AU}$ & 13 & $\$ 20245$ & 914 \\
\hline $350195153 \mathrm{2u}$ & 14 & 600522 & 915 \\
\hline 3501951 편 & 15 & $\angle B I 6.65$ & 916 \\
\hline $3501951.53 \mathrm{NM}$ & 16 & 2530049 & 917 \\
\hline $350195153 \mathrm{AM}$ & 17 & 535217 & 918 \\
\hline 35019515324 & 18 & \& 50552 & 919 \\
\hline $3501951.53 \mathrm{AN}$ & 19 & 25548.16 & 920 \\
\hline $3501951.53 \mathrm{AN}$ & $x$ & $\operatorname{cscos} 25$ & 921 \\
\hline $3501951.55 \mathrm{AM}$ & 21 & $860 \times 6.62$ & 922 \\
\hline $350195153 \mathrm{AU}$ & 22 & 45931.8 & 923 \\
\hline $350195153 \mathrm{AM}$ & 23 & 26005.72 & 924 \\
\hline $35019.51 .53 \mathrm{AM}$ & 24 & 5165.62 & 525 \\
\hline
\end{tabular}

a) Start Table

\begin{tabular}{|c|c|c|c|}
\hline DateStaing & Inder & stopi & Antamaticid \\
\hline $3 / 50195202 \mathrm{AM}$ & 0 & 43957 & 1 \\
\hline $3500195202 \mathrm{AM}$ & 1 & 4240977 & 2 \\
\hline $350195202 \mathrm{Av}$ & 2 & 445987 & 3 \\
\hline $350195202 \mathrm{AM}$ & 3 & 4453515 & $A$ \\
\hline $350195202 \mathrm{AM}$ & 4 & 44 도니 58 & 5 \\
\hline $350195202 \mathrm{AM}$ & 5 & 245642 & E \\
\hline $35 \mathrm{S01} 96202 \mathrm{AM}$ & 6 & $44005 \times 6$ & 7 \\
\hline $35.0105202 \mathrm{AM}$ & 7 & 446690 & $\theta$ \\
\hline $350195202 \mathrm{AM}$ & 日 & $48715 \times Q$ & 9 \\
\hline $350195202 \mathrm{AM}$ & 9 & 448481 & 10 \\
\hline $350195202 \mathrm{AM}$ & 10 & 4495402 & 11 \\
\hline $360195202 \mathrm{~mm}$ & 11 & 45110.87 & 12 \\
\hline $350196202 \mathrm{Au}$ & 12 & 45156.15 & 13 \\
\hline $35.0196202 \mathrm{AM}$ & 13 & 4527425 & 14 \\
\hline $350195202 \mathrm{AM}$ & 14 & 45309 o5 & 15 \\
\hline $3501952 \mathrm{m2} \mathrm{AM}$ & 15 & 4536032 & 16 \\
\hline $350195202 \mathrm{kM}$ & 16 & 45361.41 & 17 \\
\hline $350195202 \mathrm{AM}$ & 17 & 45386.06 & 10 \\
\hline $350195202 \mathrm{AM}$ & 18 & 4560761 & 19 \\
\hline $350195202 \mathrm{AM}$ & 19 & 4568989 & 20 \\
\hline $350195202 \mathrm{AM}$ & 20 & 455897 & 21 \\
\hline $350195202 \mathrm{AM}$ & 21 & 4564802 & 22 \\
\hline $350195202 \mathrm{AV}$ & 22 & 49956.45 & 23 \\
\hline $35,0195202 \mathrm{AM}$ & 23 & 4602071 & 24 \\
\hline $35 \mathrm{Ml} 95202 \mathrm{AM}$ & 24 & 4500391 & 25 \\
\hline
\end{tabular}

b) Stop Table

Figure 5-4: Sample Start and Stop Tables for the Autoscope on the Northbound Left Turn Lanes (Phase One)

\begin{tabular}{|c|c|c|c|}
\hline DafeStamp & tives: & start & Nufumatisfa \\
\hline $3 / 501100010 \mathrm{hM}$ & 0 & $443845 ?$ & 1 \\
\hline $36.011008+10 \mathrm{AM}$ & 1 & $44 a 07$ in & 2 \\
\hline $35 \mathrm{SOS} 10 \mathrm{CQ} 10 \mathrm{AM}$ & 2 & 44.89985 & 3 \\
\hline $3501100010 \mathrm{Am}$ & 3 & 4453277 & 4 \\
\hline 3501000 t0 $10 \mathrm{M}$ & 4 & 4464206 & 5 \\
\hline $35,011000+0 \mathrm{AM}$ & 5 & 4455841 & 6 \\
\hline $35 \mathrm{SAS} 10 \mathrm{Cg} 10 \mathrm{AM}$ & fi & 4464635 & 7 \\
\hline $35 \mathrm{SO} 10.0810 \mathrm{AM}$ & 7 & 4.6ED 1 & 8 \\
\hline $18 \mathrm{SOt} 10.00 \mathrm{t} / \mathrm{AM}$ & E & 4479461 & 9 \\
\hline $36.01100000 \mathrm{AM}$ & 9 & 4484499 & 10 \\
\hline 35011008 10 AM & 10 & 4484599 & 11 \\
\hline $35 \mathrm{MII} 10.0810 \mathrm{AM}$ & 11 & 4493274 & 12 \\
\hline $18.01100010 \mathrm{AM}$ & 12 & 449519 & 13 \\
\hline $36.0100000 \mathrm{Am}$ & 13 & 4506812 & 12 \\
\hline $35 \mathrm{SD} 100 \mathrm{O}$ t0 AM & 14 & 450565 & 15 \\
\hline 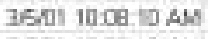 & 15 & 451539 & 16 \\
\hline $38 \mathrm{sil} 10000 \mathrm{hM}$ & 15 & 4524389 & 17 \\
\hline $3501100010 \mathrm{AM}$ & 17 & 4538399 & 18 \\
\hline $35.011008+0 \mathrm{AM}$ & 18 & 4525593 & 19 \\
\hline $35 \mathrm{Sal} 10.0810 \mathrm{Am}$ & 19 & 4530637 & 20 \\
\hline $350110000 \mathrm{hM}$ & 20 & 4531757 & 21 \\
\hline $35.011000 \div 0 \mathrm{AM}$ & 21 & 45918.11 & 22 \\
\hline $35 \mathrm{~A} 011008+10 \mathrm{AM}$ & 22 & $453+864$ & 29 \\
\hline $750110.0810 \mathrm{AM}$ & 23 & 454258 & 24 \\
\hline $3601100010 \mathrm{hm}$ & 24 & 4554953 & 25 \\
\hline
\end{tabular}

a) Start Table

\begin{tabular}{|c|c|c|c|}
\hline 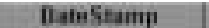 & teder & atap & Autemaficid \\
\hline $3 / 40100024 \mathrm{AW}$ & $\overline{0}$ & 4439570 & $t$ \\
\hline $35.0 \mathrm{t} 100024 \mathrm{AN}$ & 1 & $\operatorname{sen} 004$ & 2 \\
\hline 1501 10.0924 $\mathrm{AU}$ & 2 & 4532.68 & 3 \\
\hline $35 \mathrm{DI} 100824 \mathrm{MU}$ & 3 & 4453534 & 4 \\
\hline $3 / 50 t 100024 \mathrm{~km}$ & 4 & 4450021 & 5 \\
\hline $35 \mathrm{~S} 0100024 \mathrm{AM}$ & 5 & 445639 & 6 \\
\hline $7501100824 \mathrm{AU}$ & 6 & 4456554 & 7 \\
\hline $35 \mathrm{M} 100824 \mathrm{AM}$ & 7 & 44570 & 日 \\
\hline $16 \mathrm{NOt} 10.0024 \mathrm{kM}$ & 6 & 44846 & 9 \\
\hline $36.0 \div 100824$ AM & 9 & 44845.82 & 10 \\
\hline $360+100824 \mathrm{AM}$ & 10 & 4434818 & 11 \\
\hline $1501100824 \mathrm{AU}$ & 11 & $44351 . T 3$ & 12 \\
\hline $1600100024 \mathrm{NM}$ & 12 & 44954.56 & 13 \\
\hline $36.00+100024 \mathrm{AW}$ & 13 & 400039 & 14 \\
\hline $35,0+100824 \mathrm{AM}$ & 14 & $45119 t t$ & 15 \\
\hline $3501100824 \mathrm{AM}$ & 15 & 소도 15 & 16 \\
\hline $18 \mathrm{SO} 100024 \mathrm{NM}$ & 18 & 2524415 & 17 \\
\hline $3501100024 \mathrm{AM}$ & 17 & 522743 & 18 \\
\hline $35.01100824 \mathrm{AU}$ & 18 & 2520509 & 19 \\
\hline $36 \mathrm{D}$ 10.0824 $\mathrm{AN}$ & 19 & Exग7 51 & ZI \\
\hline $1501100824 \mathrm{NM}$ & $x$ & 25317 84 & 21 \\
\hline $3501100024 \mathrm{AM}$ & 21 & 5316.36 & 22 \\
\hline 360 t 100924 LU & 22 & 435514 & 29 \\
\hline $3501100824 \mathrm{AM}$ & 23 & $54 \mathrm{~d} 202$ & 24 \\
\hline $16 \mathrm{D} 110.0024 \mathrm{AM}$ & 24 & S5580.14 & 7 \\
\hline
\end{tabular}

b) Stop Table

Figure 5-5: Sample Start and Stop Tables for the Video-Trak 905 on the Southbound Left Turn Lanes (Phase One) 


\begin{tabular}{|c|c|c|c|}
\hline DaieStainp & Intex & stant & Auminaticis \\
\hline $360140402 \mathrm{PM}$ & 0 & $443930 ?$ & \\
\hline $3 / 5.01 \pm 0402$ PM & 1 & 4420633 & \\
\hline $1501+04 \mathrm{OP} \mathrm{PM}$ & 2 & 4440959 & \\
\hline $35 \mathrm{SI} 404$ प2 PM & 3 & 4454179 & \\
\hline $350140402 \mathrm{PM}$ & 2 & 44557.91 & \\
\hline $35.0140402 \mathrm{PM}$ & 5 & 4464538 & \\
\hline $35 \mathrm{DI} 404 \mathrm{O2} \mathrm{PM}$ & 6 & 44799 43 & \\
\hline 3501404 प2 $\mathrm{PM}$ & 7 & 44931.54 & \\
\hline $360140402 \mathrm{PM}$ & 8 & 45087 63 & \\
\hline $350140402 \mathrm{PM}$ & 9 & $\$ 516353$ & 10 \\
\hline $3501+04 \mathrm{C2} \mathrm{PM}$ & 10 & $45 \times 6352$ & 11 \\
\hline 35 II $\& 04$ प2 PM & 11 & 45305 ES & 12 \\
\hline 350140402 PM & 12 & 45357,05 & t \\
\hline $36.01<0402 \mathrm{PM}$ & 13 & 4544516 & $t$ \\
\hline $35 \mathrm{DO}+04$ 02 PU & 14 & $255+325$ & $t$ \\
\hline 3501404 प2 PM & 15 & $45331 \mathrm{B9}$ & i \\
\hline $150140402 \mathrm{PM}$ & 16 & 45006 ह 5 & 1 \\
\hline $95001<0402 \mathrm{PM}$ & 17 & 4516873 & t \\
\hline $3501<0402 \mathrm{PM}$ & 18 & 460907 & $t$ \\
\hline $35 \mathrm{MI} 404 \mathrm{~W} \mathrm{PM}$ & 19 & 4521958 & 2 \\
\hline $350120402 \mathrm{PM}$ & 20 & 2.23564 & 2 \\
\hline $35.03<0402 \mathrm{PMA}$ & 21 & 9.271 .32 & \\
\hline $3501<0402 \mathrm{PM}$ & 22 & 463963 & 23 \\
\hline 3501404 व2 $\mathrm{PM}$ & 23 & 45444.64 & 24 \\
\hline 3501404 .12 PM & 24 & AERा4 27 & 2 \\
\hline
\end{tabular}

a) Start Table

\begin{tabular}{|c|c|c|c|}
\hline DaieStaing & Mndex & strip & Autumaticil \\
\hline 3/50136639. PM & 0 & 42966.7 & 1 \\
\hline 3.5 .01350 .33 PM & 1 & 4400763 & 2 \\
\hline $150135633 \mathrm{PM}$ & 2 & 4453525 & 3 \\
\hline $35 \mathrm{MI} 356.33 \mathrm{PM}$ & 3 & 4454974 & 4 \\
\hline $350136 t 35 \mathrm{PPA}$ & 2 & 446269 & 5 \\
\hline $35.01365,33 \mathrm{PM}$ & 5 & 4465723 & 6 \\
\hline $35 \mathrm{Sn1} 35533 \mathrm{PM}$ & 6 & $44 B A 7+48$ & 7 \\
\hline $3501355.33 \mathrm{PM}$ & 7 & 4495428 & 8 \\
\hline $3501366.33 \mathrm{PM}$ & 8 & astivat. & 9 \\
\hline $3500136633 \mathrm{Pu}$ & 9 & 4515479 & 10 \\
\hline $350135833 \mathrm{Pu}$ & 10 & 4527353 & 11 \\
\hline $35 \mathrm{MI} 356$ 33 PM & 11 & 4530125 & 12 \\
\hline $15001366.39 \mathrm{PM}$ & 12 & 45005.44 & 13 \\
\hline $35.0135633 \mathrm{PM}$ & 13 & 45451,2 & 14 \\
\hline $35 \mathrm{N01} 35633 \mathrm{Pu}$ & 14 & 4552943 & 15 \\
\hline $350135533 \mathrm{PM}$ & 15 & $459 c 591$ & 16 \\
\hline $15013653 \mathrm{PH}$ & 15 & 5002042 & 17 \\
\hline $35.0135633 \mathrm{Pm}$ & 17 & 2000091 & 18 \\
\hline $350135033 \mathrm{Pu}$ & 18 & $2: 20329$ & 19 \\
\hline $35 \mathrm{MI1} 356.33 \mathrm{PM}$ & 19 & कर2221 & 20 \\
\hline $3501356.33 \mathrm{PMA}$ & 20 & 5.230. & 21 \\
\hline $35.0136639 \mathrm{PMA}$ & 21 & 200942 & 22 \\
\hline $750135533 \mathrm{PM}$ & 22 & aste.72 & 33 \\
\hline $150135633 \mathrm{FM}$ & 23 & 454463 & 24 \\
\hline $3501356.33 \mathrm{PM}$ & 24 & 2574539 & 25 \\
\hline
\end{tabular}

b) Stop Table

Figure 5-6: Sample Start and Stop Tables for the Inductive Loops on the Southbound Left Turn Lanes (Phase One)

\begin{abstract}
$\underline{\text { Results Screen }}$
Now that the possible discrepancies between inductive loops and video detectors and the difference between "error" and "discrepancy" have been touched on briefly in Chapter 4, the reader should already know a little about what is available through the Results Screen (Figure 5-2) by reading the captions on the various buttons. Each group of buttons and their functions will be explained in this section.
\end{abstract}

The Results Screen is a graphical representation of the calculations that have been performed by the software. The tool that was used to produce the graphs is the MSChart tool in Visual Basic. This charting tool has some limitations when plotting bar charts with a time-based X-axis. For this reason, many of the charts produced by this program are simply preliminary graphs that are simple in nature. If the user would like to have more sophisticated graphs, 
he/she can upload the import the necessary Access tables into Excel, or any other spreadsheet program and produce the graphs that they desire.

One may notice that there are three groups of buttons available on the results screen. The titles of these groups are Individual Discrepancies, Cumulative Discrepancies, and Likelihood Estimates. Each of these groups characterize the different ways of displaying the discrepancies between the inductive loops and the video detectors.

\section{Individual Discrepancies}

Within this group, there are two buttons - one for the Econolite, and one for the Peek video detector. After pressing one of these buttons, a graph is shown that shows each individual discrepancy. A sample of these graphs is shown in (Figure 5-7). The LOV1 discrepancy (overdetection, or false calls) is plotted as a positive discrepancy. The L1V0 discrepancy (underdetection; missed or dropped calls) is plotted as a negative discrepancy. By plotting in this fashion, both L0V1 and L1V0 discrepancies can be shown on one graph. The user should be aware that the MSChart tool used to plot these graphs will not plot the X-axis to a time scale. The graph shows every single error that is greater than the blanking band which the user previously shown. It does not show times when there is no error. The $\mathrm{X}$-axis is labeled in seconds since midnight of the first day of data collection. If the user would like the X-axis to be scaled off, the appropriate Access table may be imported to a spreadsheet program and plotted.

\section{Cumulative Discrepancies}

These graphs show exactly what one would expect. They are an accumulation of the individual discrepancies plotted in the previously explained graph. This graph is plotted to a time scale, and therefore may be an aid in giving an indication of the time(s) when there was a problem that should be checked into more closely. An example of this type of graph is shown in Figure $5-8$. The main point that should be made in reference to the cumulative 
discrepancy graphs is that they can sometimes mask potential problems because of the magnitude of the $\mathrm{Y}$-axis scale. They are also very unforgiving in the sense that if there is one large error, and the rest of the performance is good, the magnitude of the $\mathrm{Y}$-axis scale is deceiving. In other words, these graphs should only be used for a quick overview of the data. It should never be taken as the last word without further investigation.

\section{Available Craphs:}
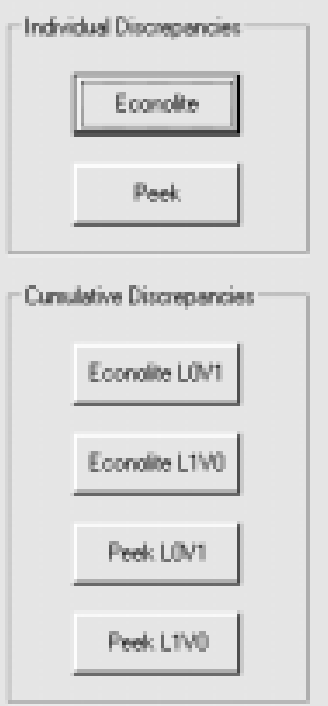

- Likelinod Eitinghes-

Ecomeline

$P|=0| L=1 \mid$ PYNo1 IL $=0 \mid$ Peek.

Econolite Individual Discrepancies (LOV1+, L1V0-)

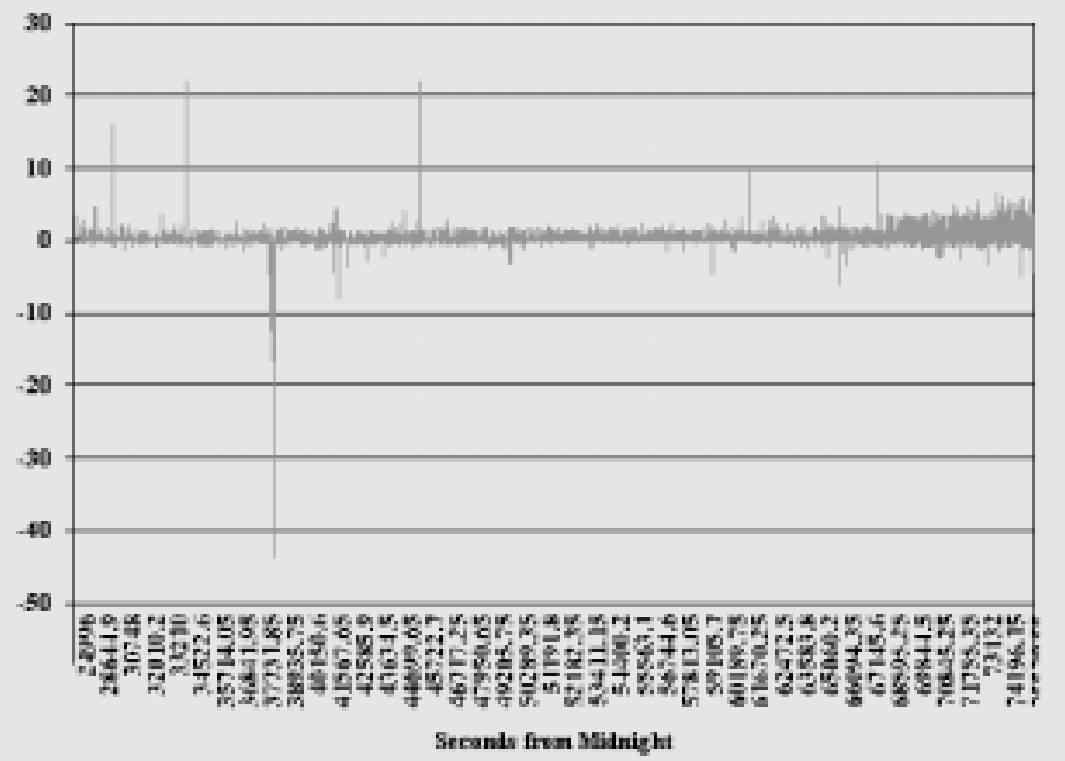

Pick. a diterent evaluivion trine

End Progm

Figure 5-7: Example of Individual Discrepancies Graph

Likelihood Estimates

The purpose of these graphs is to have a graphical representation of the probability that a certain type of discrepancy is taking place. An example of the Likelihood Estimates graph can be seen in Figure 5-9. The values used to populate these graphs are calculated using the equations given in Chapter 4 . 


\section{Q. Fiexulix}

Available Craphs:

-Indridus Decrepencies

Econclite

Peek

- Cundutive Ditarepencies-

Econoline LOV1

Econoline L1WD

Feck LQV1

Peek L1W0

- Likelhood Eingles

Econdine

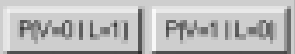

Peek.

$P W=0 \mid L=1] \quad F(N=1|L=0|$
Eeonolite Cumulative L1Vo Diserepancy

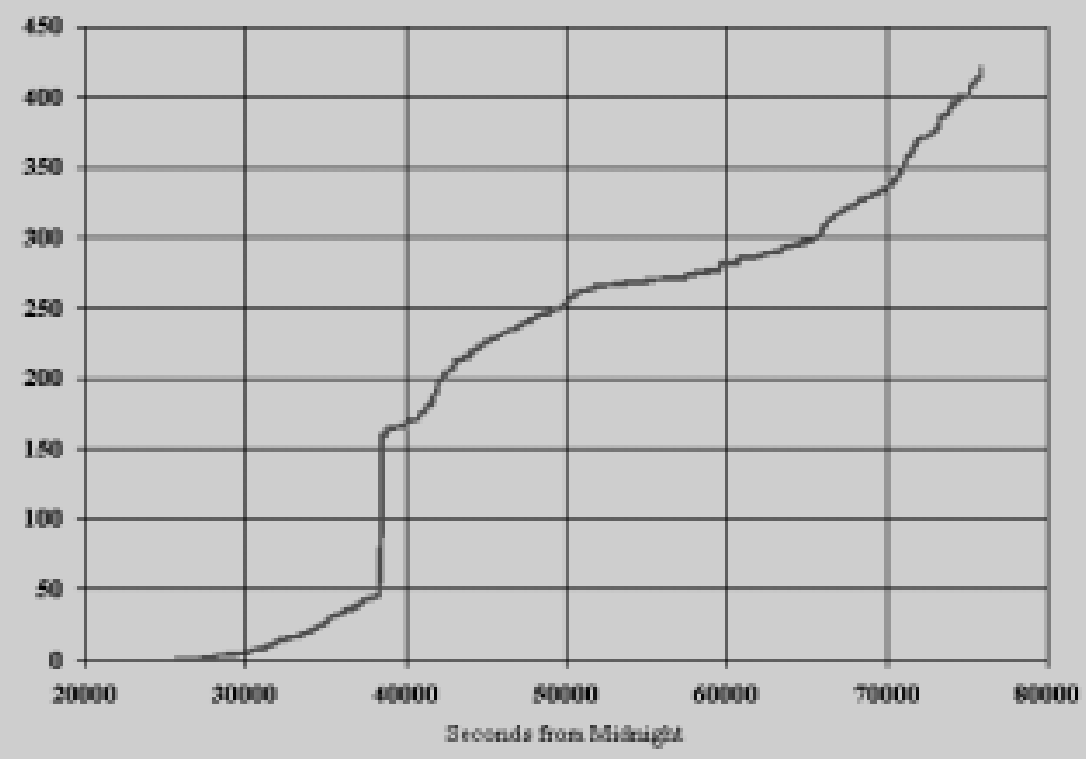

Rick. a diterent ewaluien tire

Figure 5-8: Example of Cumulative Discrepancies Graph 


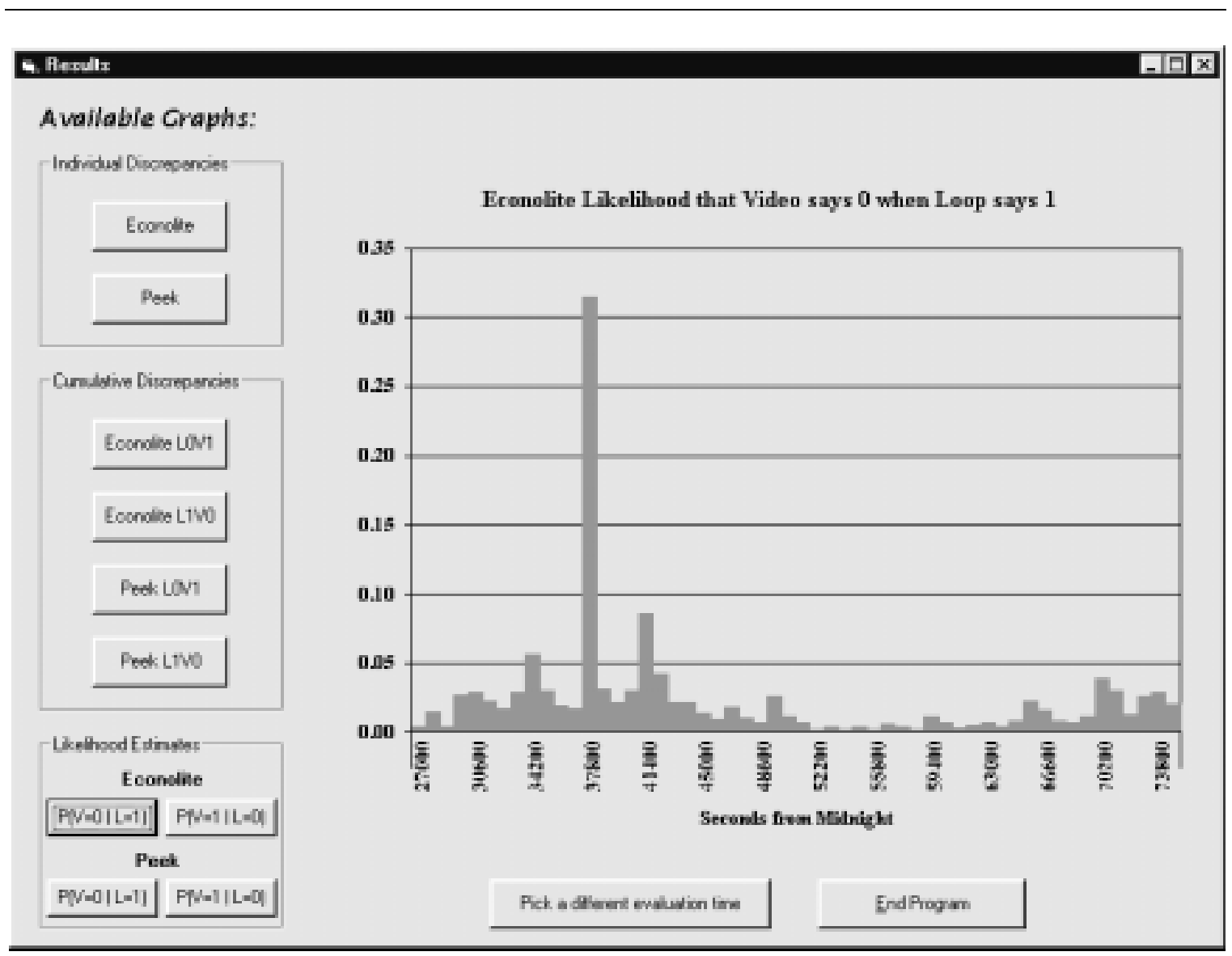

Figure 5-9: Example of Likelihood Estimate Graph

\section{Concluding Remarks}

With the amount of data that is collected for this research to be possible, it is essential that there be some sort of tool which aids in the data reduction, calculations, and gives a quick glance at the data in a graphical form. This is the reason for which this software was written. When looking at each of the graphs produced by the software, it is obvious that there are some limitations. Despite these limitations, the calculations that have been performed can be imported to a spreadsheet program and plotted in a more professional manner. 


\section{CHAPTER 6 - ANALYSIS OF LOV1 AND L1VO DISCREPANCIES}

\section{Chapter Overview}

The objective of this chapter is to relate the results of the evaluation efforts performed for this research. Three of the data collection days have been thoroughly analyzed and are presented here. The days that are shown here are representative of overcast conditions, night rain conditions, and partly sunny conditions. The overcast and night rain conditions were collected before the final calibration of each of the video detection systems. The partly sunny conditions were collected after the final calibration was performed. Therefore, by comparing the data from the overcast condition to the partly sunny condition, some idea is given of the impact of recalibration on each of the systems. Also presented in this chapter is the effect of calibration on the activation distances of video detection during the night. The activation distance is the distance a vehicle is from the stop bar when it becomes detected by the video detection system. For the sake of comparison, the activation distances during the day are also shown after recalibration. This gives the reader an idea of the dramatic effect vehicle headlights have on video detection at night.

\section{Blanking Band}

The system has been set up so that it reports each discrepancy, no matter the size. It is obvious that it would be impossible to have the video detectors and inductive loops so perfectly lined up that they indicate each vehicle's presence exactly the same. This is because the video detector detects objects optically, 
and the inductive loop detects objects magnetically. When objects are detected magnetically, the actually dimensions of the vehicle do not affect the ability of the inductive loops to properly detect vehicles. With the video detector, however, the height of the vehicle does affect the ability of the detector to detect vehicles properly. If a vehicle is tall, the smaller the angle between the ground and the camera, the longer that vehicle will be detected by the video detector. It may also cause occlusion, which means that vehicles behind the taller vehicle may be included in the same detection as the tall vehicle, because the video detector is not able to distinguish between the end of the tall vehicle and the front of the next vehicle.

It is for the above reasons that a blanking band has been used for evaluation purposes. The purpose of this blanking band is to remove all discrepancies smaller than a user-defined value from the evaluation. For the purpose of this report, a blanking band of 2 seconds was used. Therefore, any discrepancy less than 2 seconds will be removed from the evaluation, as discrepancies of this size are not usually of importance.

There is a case when these small discrepancies could affect the proper operation of an intersection. These discrepancies could occur when vehicles have a green signal and are traveling quickly. If the video detector drops a detection in the middle of a platoon of vehicles, or if one vehicle is not detected, this could cause the green signal to be terminated prematurely. In this case, the discrepancy would most certainly be less than 2 seconds. These would not be identified in this evaluation.

\section{Data Collection}

Data collection was performed on several occasions for this research in approximately 24-hour segments. Each time a different weather condition was 
expected, another data collection attempt was made. In this context, data collection consists of the capture of all four approaches at the instrumented intersection on digital video in RealVideo format with text overlay and the collection of all video detection events, all inductive loop detection events, and signal states in a database. All calculations can be performed on the data in the database, and these calculations may be ground truthed against the text overlay on the video.

\section{Night Rain Conditions (Before Recalibration)}

The first data collection presented in this chapter is from 4:00 PM on February $23-3: 00$ PM on February 24, 2001. During the night, it began raining around 2:00 AM, and continued raining until around 7:00 AM. Figure 6-1 through Figure 6-8 show the individual L0V1 and L1V0 discrepancies of both Video Detection System 1 and Video Detection System 2 for all lanes serving the eight phases at this intersection. Table 6-1 through Table 6-8 give the hourly discrepancy totals, in seconds, of System 1 and System 2 for all eight phases, as well. Each of the figures has been reviewed, and the largest of discrepancies has been ground truthed according to the text overlay on the digital video recorded during data collections. In order to do this, whenever there is a large spike in the discrepancy graph, the video is consulted to determine whether the discrepancy was caused by the inductive loops or the video detectors.

Most discrepancies are caused by the video detectors, but one can see in Figure 6-2 that there are several times when the inductive loops register a call when there is no vehicle present, and thus cause L1V0 errors. This is sometimes caused by faulty inductive loops, and sometimes by oversensitive loops registering a call because of a large vehicle in an adjacent lane served by another phase. 


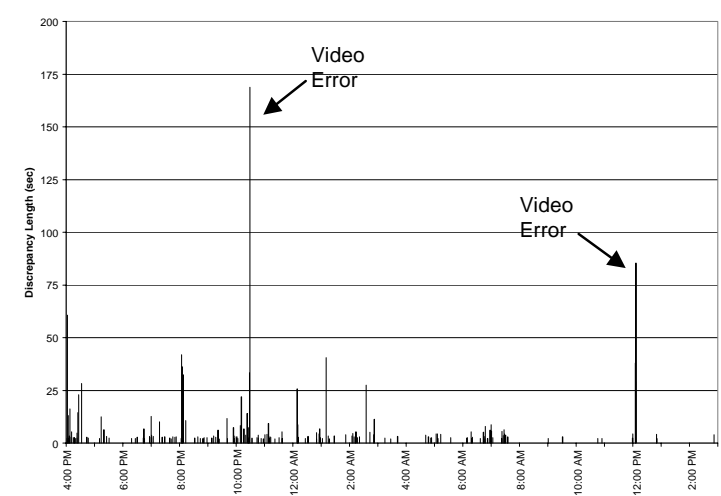

a) System 1 LOV1

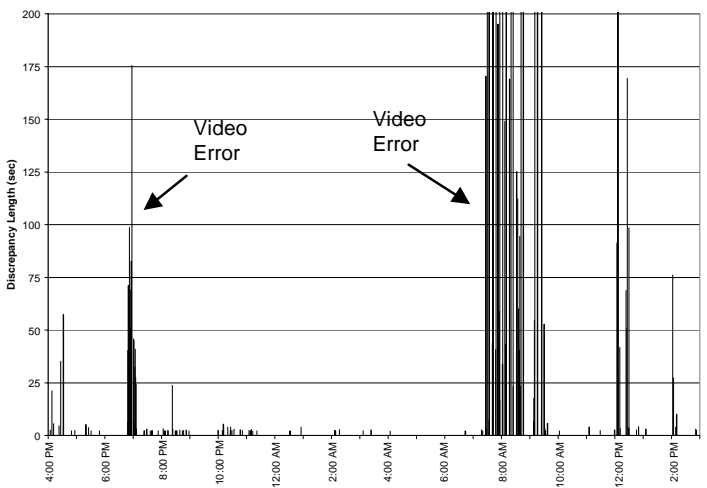

c) System 2 LOV1

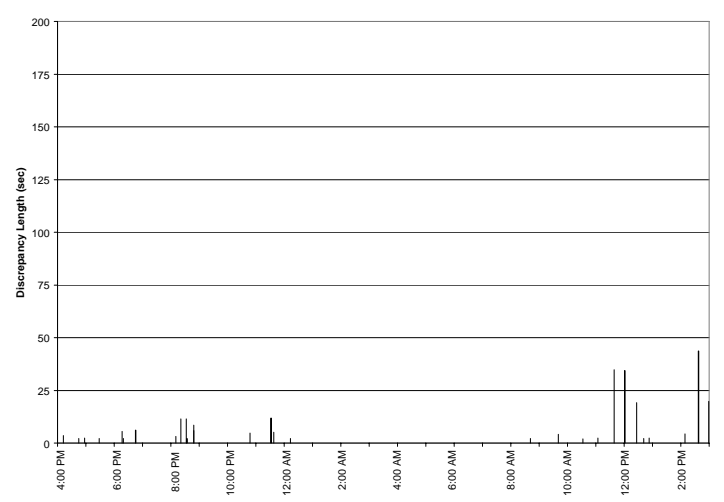

b) System 1 L1V0

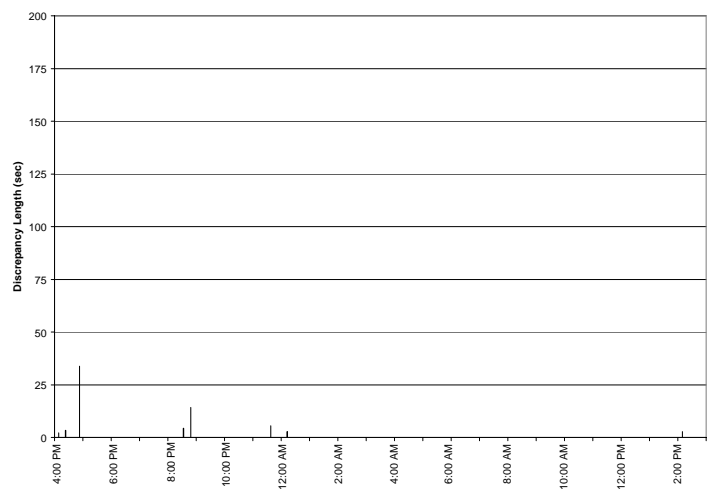

d) System 2 L1V0

Figure 6-1: Phase 1, Blanking Band $=2$ sec., February $23 \& 24,2001$, Rain 


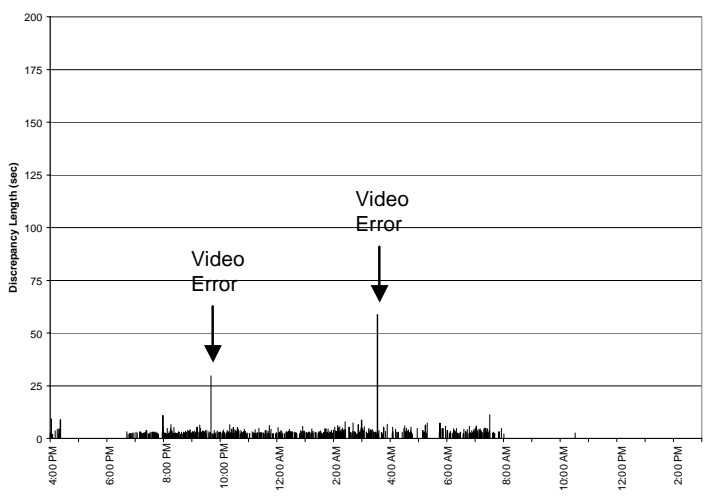

a) System 1 LOV1

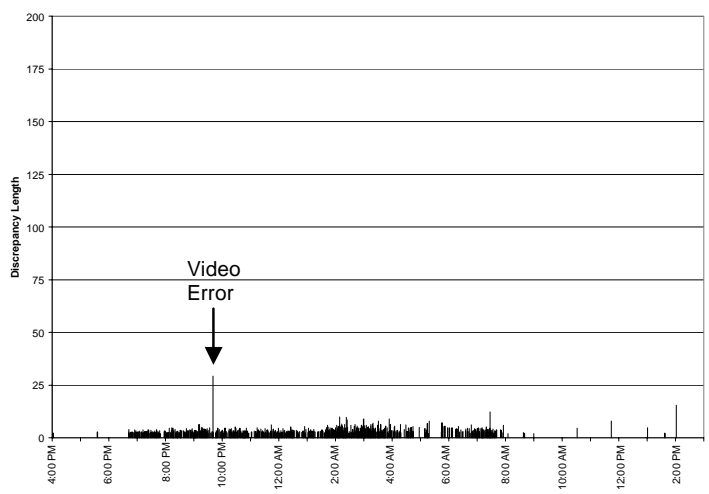

c) System 2 LOV1

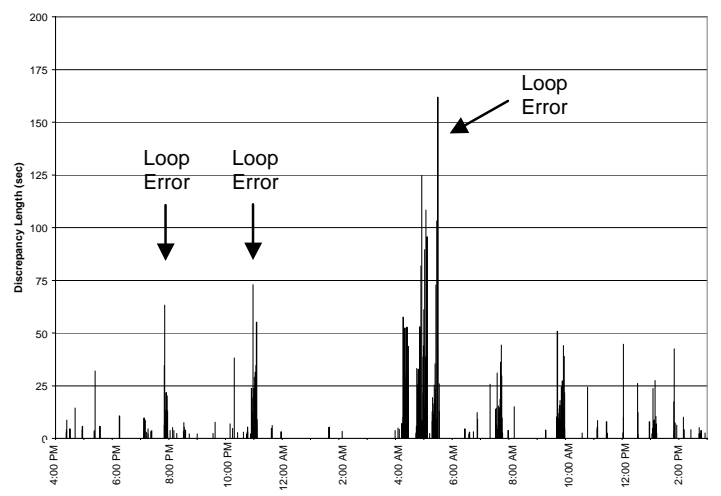

b) System 1 L1V0

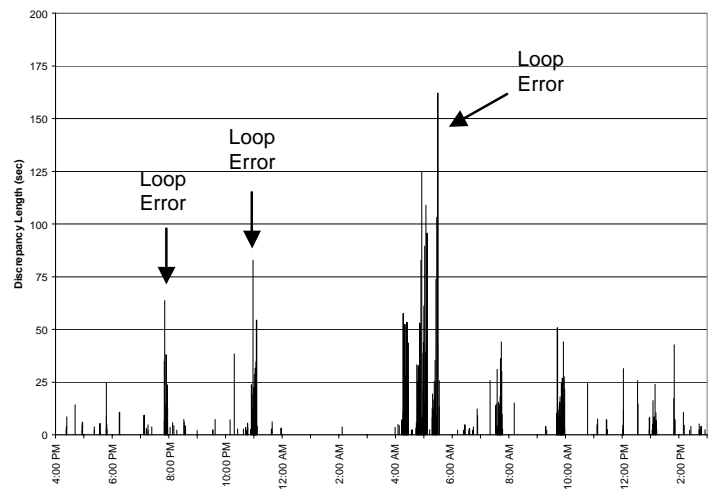

d) System 2 L1V0

Figure 6-2: Phase 2, Blanking Band = 2 sec., February $23 \& 24,2001$, Rain 


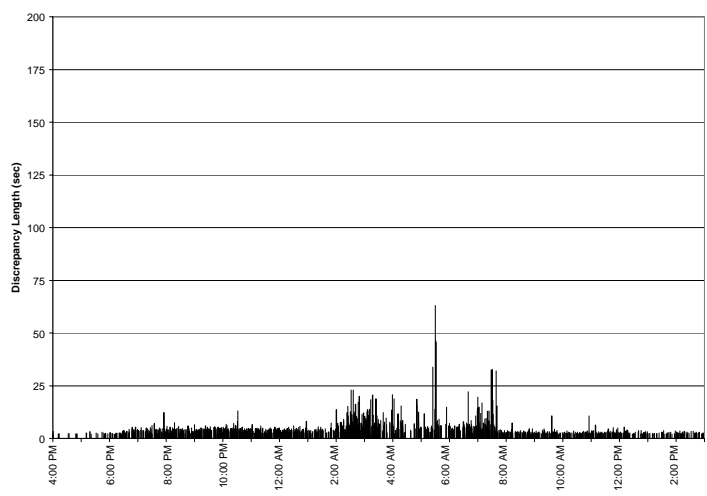

a) System 1 LOV1

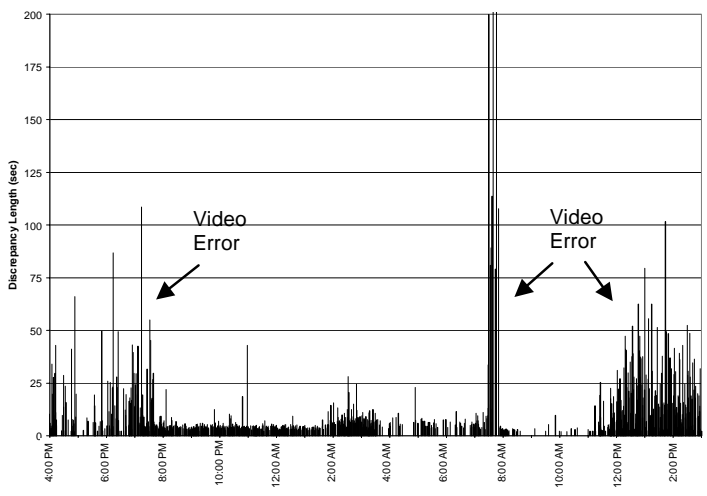

c) System 2 LOV1

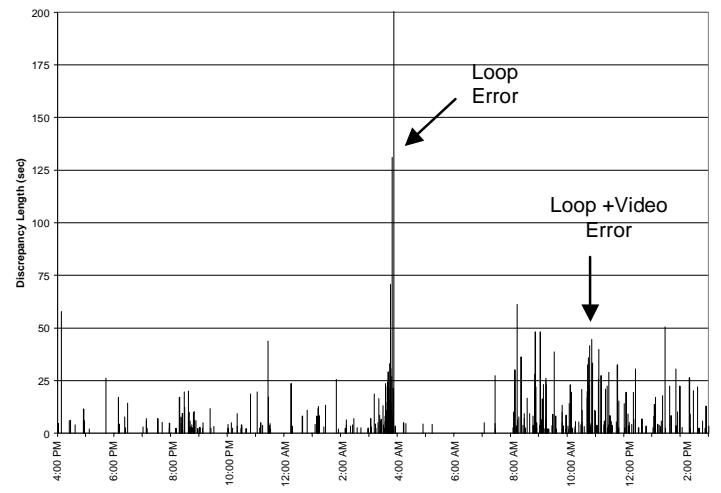

b) System 1 L1V0

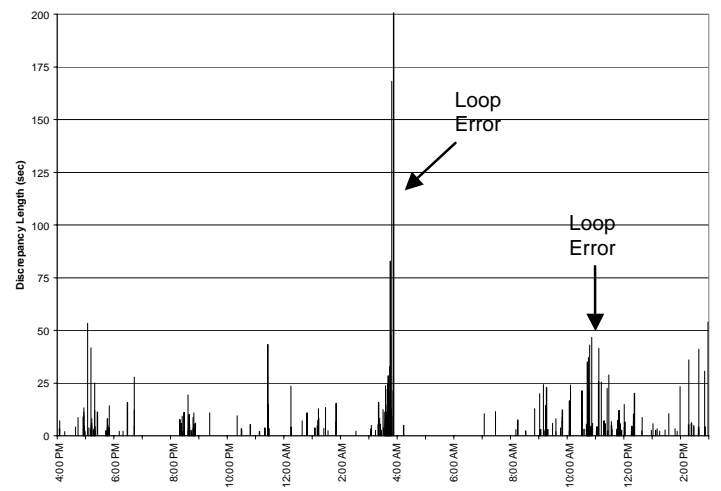

d) System 2 L1V0

Figure 6-3: Phase 3, Blanking Band = 2 sec., February $23 \& 24$, 2001, Rain 


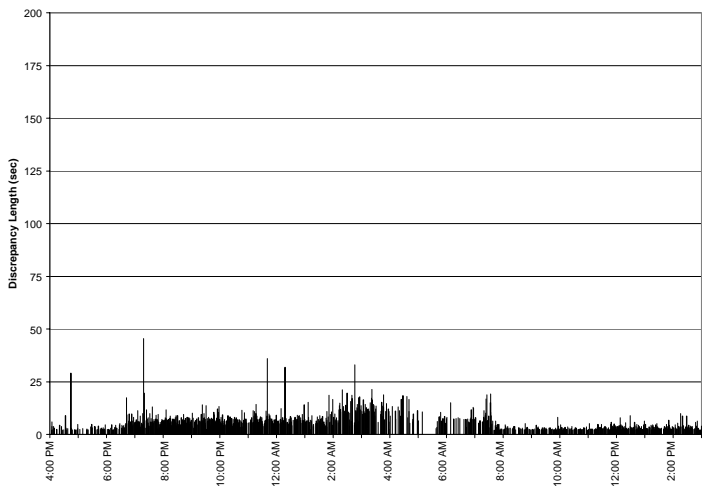

a) System 1 LOV1

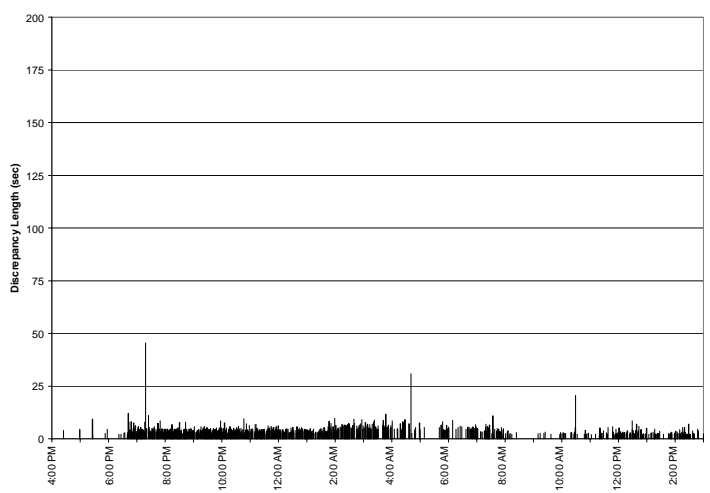

c) System 2 LOV1

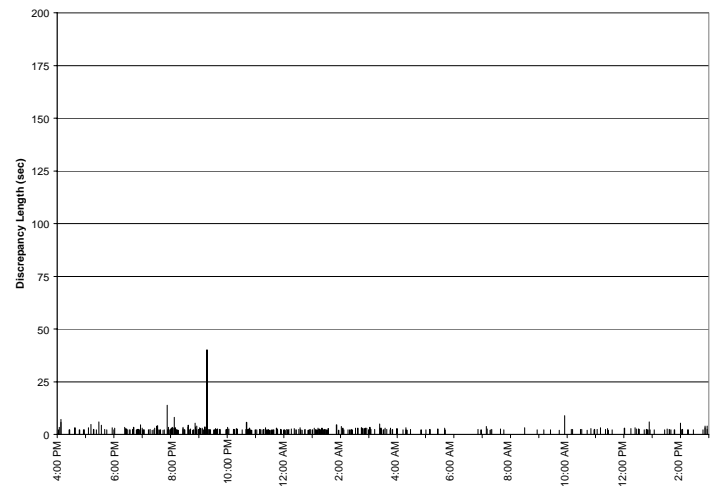

b) System 1 L1V0

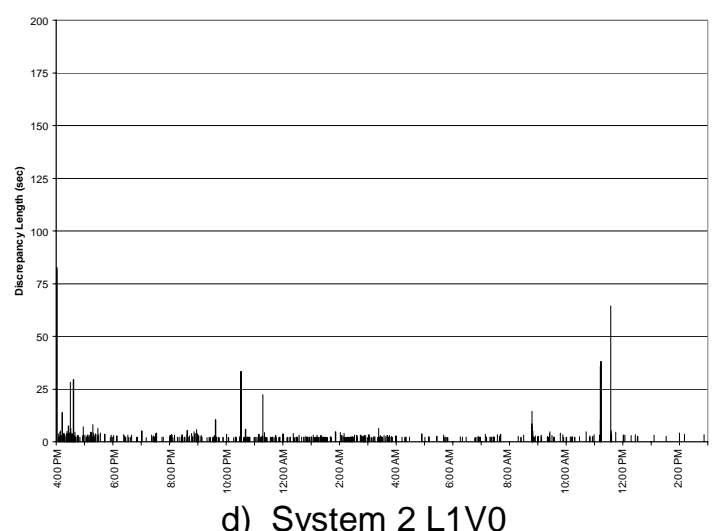

d) System 2 L1V0

Figure 6-4: Phase 4, Blanking Band = 2 sec., February $23 \& 24$, 2001, Rain 


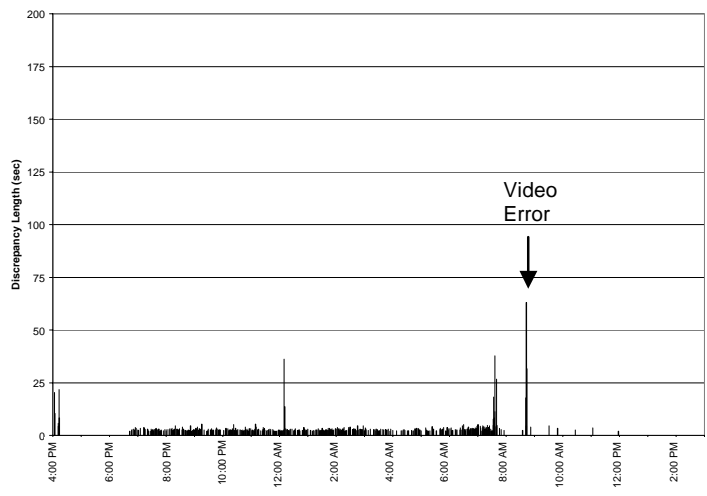

a) System 1 LOV1

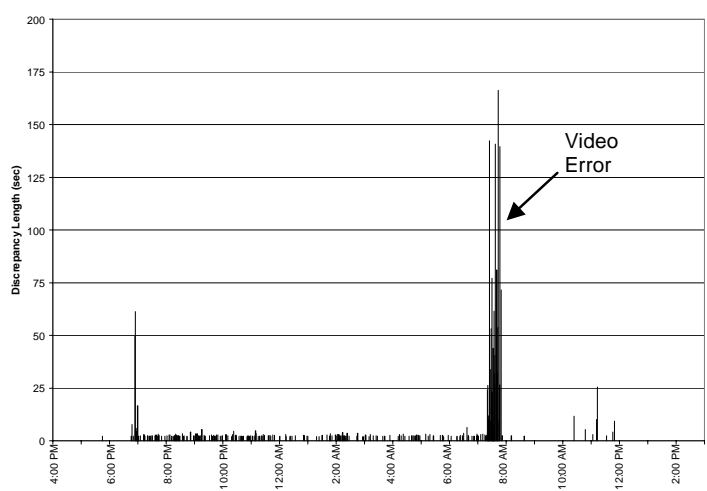

c) System 2 LOV1

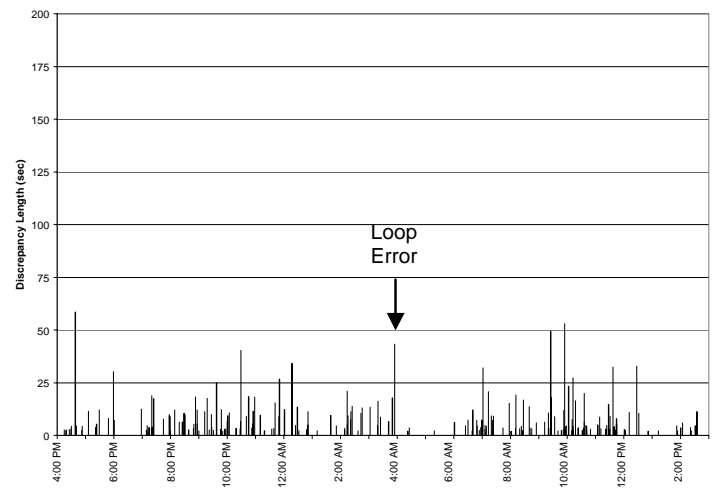

b) System 1 L1V0

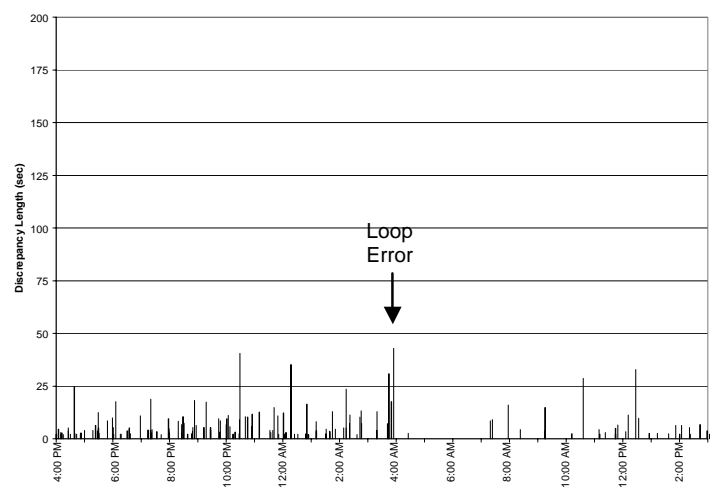

d) System 2 L1V0

Figure 6-5: Phase 5, Blanking Band = 2 sec., February $23 \&$ 24, 2001, Rain 


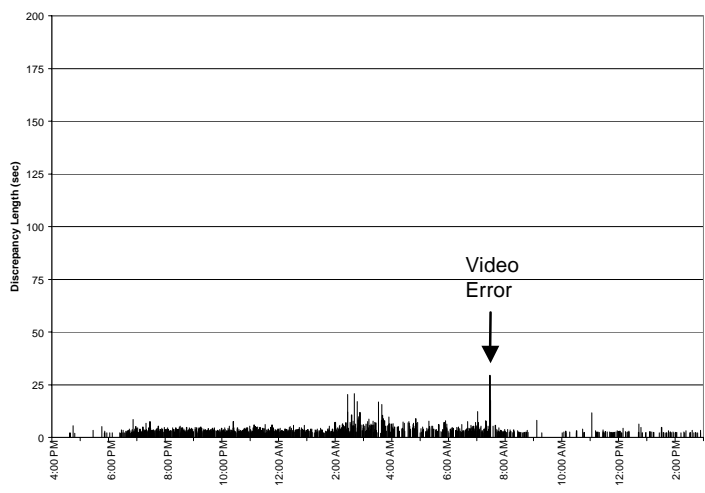

a) System 1 LOV1

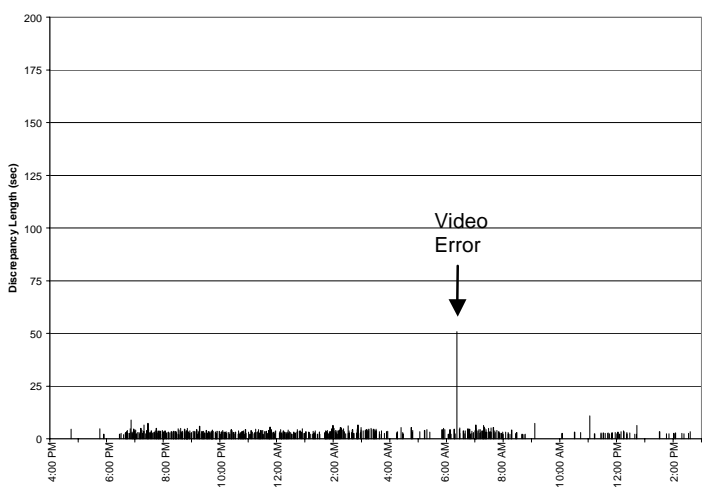

c) System 2 LOV1

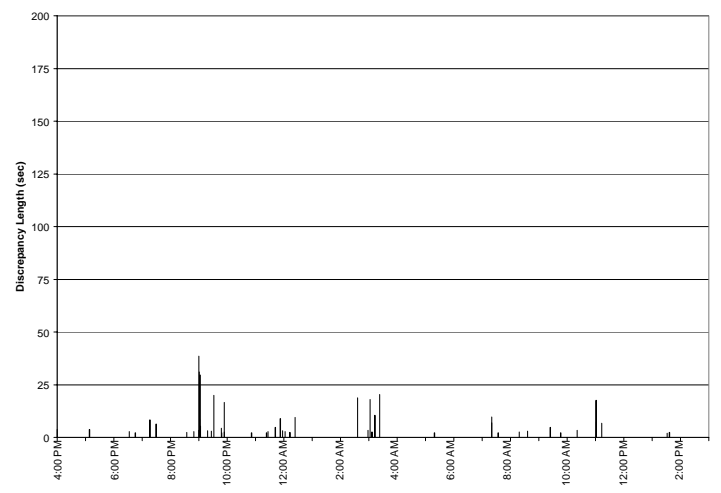

b) System 1 L1V0

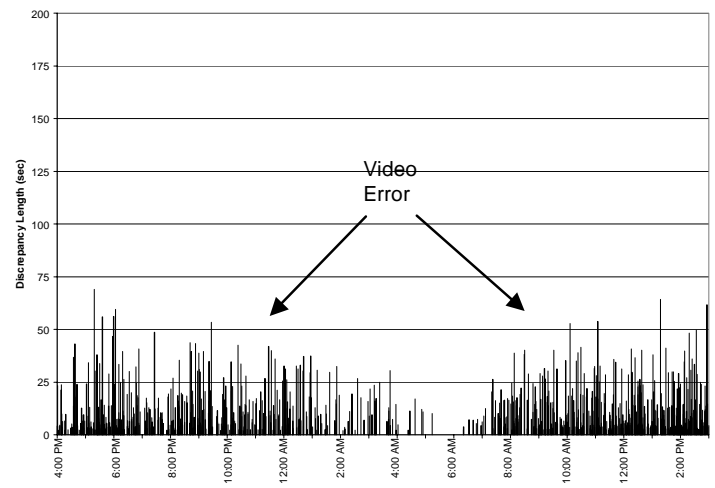

d) System 2 L1V0

Figure 6-6: Phase 6, Blanking Band = 2 sec., February 23 \& 24, 2001, Rain 


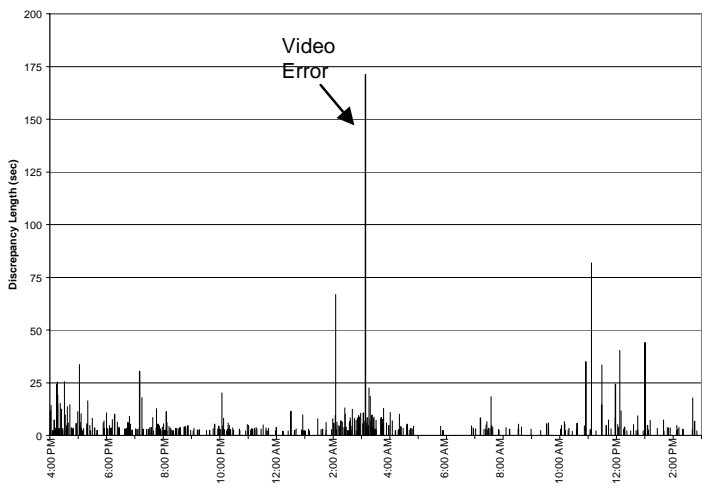

a) System 1 LOV1

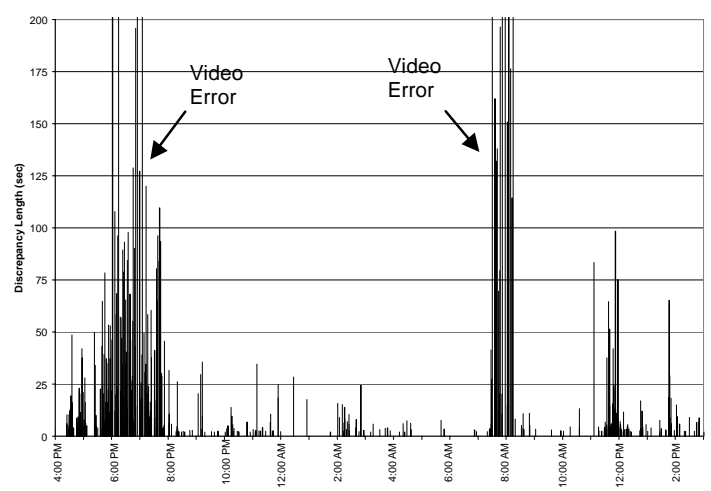

c) System 2 LOV1

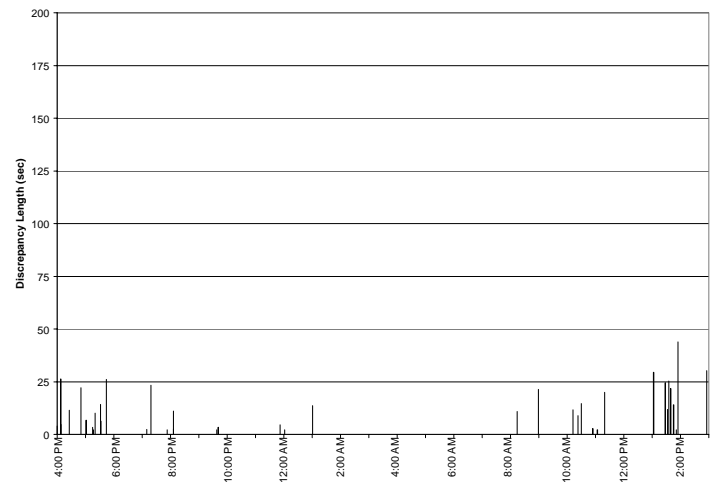

b) System 1 L1V0

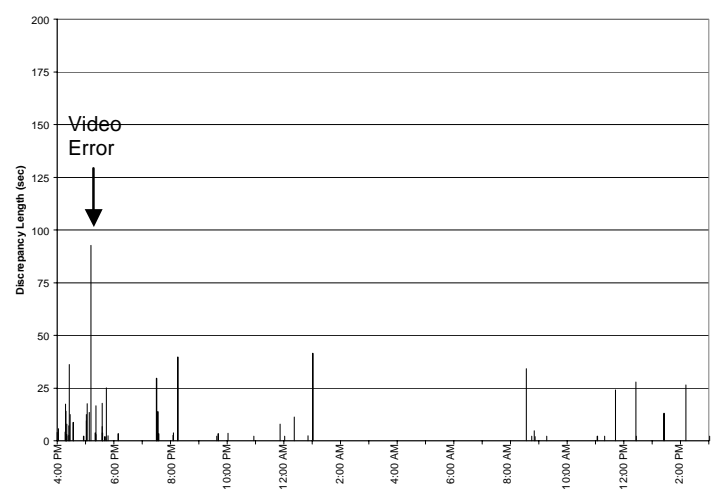

d) System 2 L1V0

Figure 6-7: Phase 7, Blanking Band $=2$ sec., February $23 \& 24,2001$, Rain 


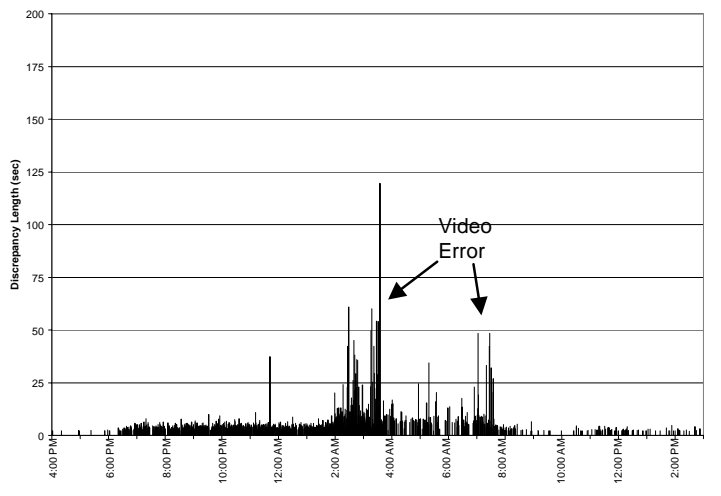

a) System 1 LOV1

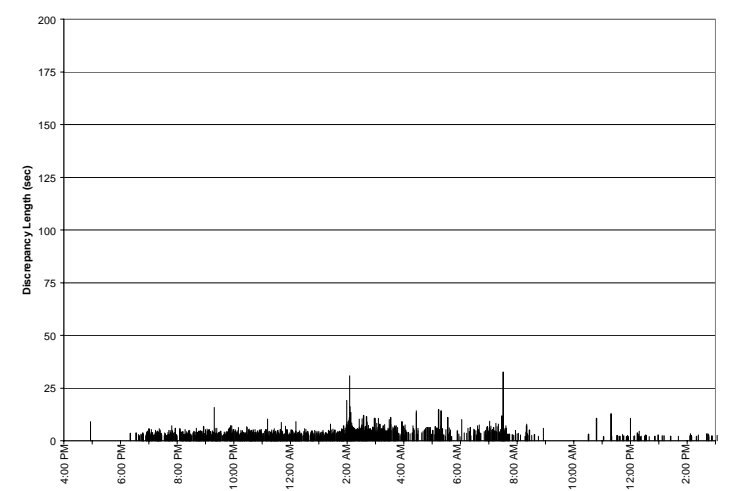

c) System 2 LOV1

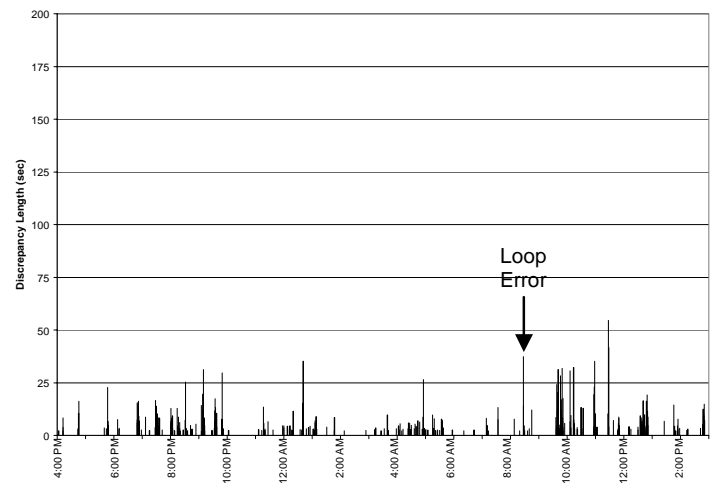

b) System 1 L1V0

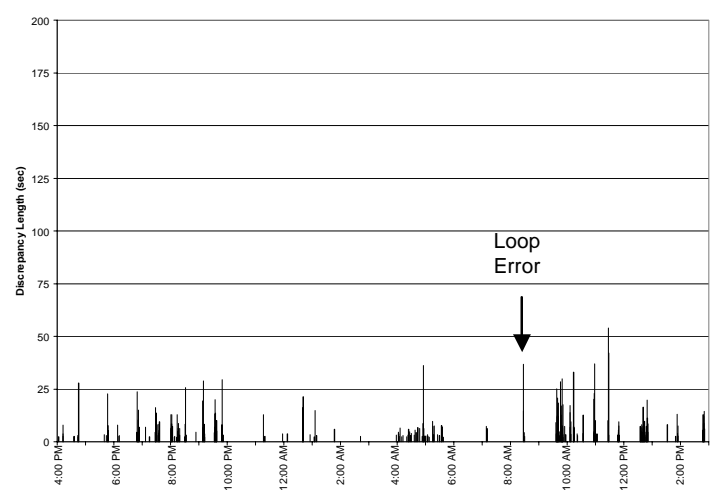

d) System 2 L1V0

Figure 6-8: Phase 8, Blanking Band = 2 sec., February $23 \& 24,2001$, Rain 
Table 6-1: Hourly Discrepancy Totals for Phase 1, Blanking Band = $2 \mathrm{sec}$, February 23 \& 24, 2001, Rain

\begin{tabular}{|c|r|r|r|r|}
\cline { 2 - 5 } \multicolumn{1}{c|}{} & \multicolumn{2}{c|}{ System 1 } & \multicolumn{2}{c|}{ System 2 } \\
\hline Hour Beginning & L0V1 & L1V0 & L0V1 & L1V0 \\
\hline \hline $4: 00$ PM & 196 & 8 & 131 & 39 \\
\hline $5: 00$ PM & 26 & 2 & 13 & 0 \\
\hline $6: 00$ PM & 22 & 14 & 647 & 0 \\
\hline $7: 00$ PM & 53 & 0 & 253 & 0 \\
\hline $8: 00$ PM & 194 & 42 & 58 & 19 \\
\hline $9: 00$ PM & 45 & 0 & 0 & 0 \\
\hline $10: 00$ PM & 296 & 5 & 40 & 0 \\
\hline $11: 00$ PM & 38 & 17 & 14 & 6 \\
\hline $12: 00$ AM & 65 & 2 & 6 & 3 \\
\hline $1: 00$ AM & 55 & 0 & 0 & 0 \\
\hline $2: 00$ AM & 72 & 0 & 7 & 0 \\
\hline $3: 00$ AM & 8 & 0 & 7 & 0 \\
\hline $4: 00$ AM & 12 & 0 & 2 & 0 \\
\hline $5: 00$ AM & 20 & 0 & 0 & 0 \\
\hline $6: 00$ AM & 53 & 0 & 4 & 0 \\
\hline $7: 00$ AM & 40 & 0 & 2111 & 0 \\
\hline $8: 00$ AM & 0 & 2 & 2931 & 0 \\
\hline $9: 00$ AM & 5 & 4 & 1279 & 0 \\
\hline $10: 00$ AM & 4 & 2 & 2 & 0 \\
\hline $11: 00$ AM & 7 & 37 & 9 & 0 \\
\hline $12: 00$ PM & 159 & 58 & 796 & 0 \\
\hline $1: 00$ PM & 0 & 0 & 3 & 0 \\
\hline $2: 00$ PM & 4 & 68 & 123 & 3 \\
\hline \hline Total & 1373 & 262 & 8436 & 69 \\
\hline
\end{tabular}


Table 6-2: Hourly Discrepancy Totals for Phase 2, Blanking Band = $2 \mathrm{sec}$, February 23 \& 24, 2001, Rain

\begin{tabular}{|c|r|r|r|r|}
\cline { 2 - 5 } \multicolumn{1}{c|}{} & \multicolumn{2}{c|}{ System 1 } & \multicolumn{2}{c|}{ System 2 } \\
\hline Hour Beginning & \multicolumn{1}{c|}{ L0V1 } & \multicolumn{1}{c|}{ L1V0 1} & \multicolumn{1}{c|}{ L1V0 } \\
\hline \hline 4:00 PM & 37 & 46 & 4 & 46 \\
\hline $5: 00 \mathrm{PM}$ & 0 & 47 & 3 & 63 \\
\hline $6: 00 \mathrm{PM}$ & 17 & 11 & 46 & 11 \\
\hline $7: 00 \mathrm{PM}$ & 110 & 266 & 139 & 261 \\
\hline 8:00 PM & 148 & 43 & 178 & 39 \\
\hline 9:00 PM & 167 & 12 & 212 & 14 \\
\hline $10: 00 \mathrm{PM}$ & 170 & 216 & 174 & 221 \\
\hline $11: 00 \mathrm{PM}$ & 90 & 288 & 153 & 280 \\
\hline $12: 00 \mathrm{AM}$ & 131 & 0 & 148 & 0 \\
\hline $1: 00 \mathrm{AM}$ & 131 & 5 & 176 & 0 \\
\hline $2: 00 \mathrm{AM}$ & 177 & 3 & 305 & 0 \\
\hline $3: 00 \mathrm{AM}$ & 209 & 4 & 298 & 4 \\
\hline $4: 00 \mathrm{AM}$ & 69 & 1228 & 114 & 1226 \\
\hline $5: 00 \mathrm{AM}$ & 61 & 1081 & 62 & 1085 \\
\hline $6: 00 \mathrm{AM}$ & 127 & 34 & 129 & 41 \\
\hline $7: 00 \mathrm{AM}$ & 155 & 381 & 194 & 371 \\
\hline 8:00 AM & 2 & 15 & 6 & 15 \\
\hline $9: 00 \mathrm{AM}$ & 0 & 481 & 2 & 471 \\
\hline $10: 00 \mathrm{AM}$ & 3 & 27 & 4 & 24 \\
\hline $11: 00 \mathrm{AM}$ & 0 & 29 & 8 & 26 \\
\hline $12: 00 \mathrm{PM}$ & 0 & 106 & 0 & 104 \\
\hline $1: 00 \mathrm{PM}$ & 0 & 195 & 7 & 174 \\
\hline $2: 00 \mathrm{PM}$ & 0 & 38 & 15 & 39 \\
\hline \hline Total & 1801 & 4557 & 2380 & 4515 \\
\hline
\end{tabular}


Table 6-3: Hourly Discrepancy Totals for Phase 3, Blanking Band = 2 sec., February 23 \& 24, 2001, Rain

\begin{tabular}{|c|r|r|r|r|}
\cline { 2 - 5 } \multicolumn{1}{c|}{} & \multicolumn{2}{c|}{ System 1 } & \multicolumn{2}{c|}{ System 2 } \\
\hline Hour Beginning & L0V1 & L1V0 & L0V1 & L1V0 \\
\hline \hline 4:00 PM & 19 & 109 & 513 & 79 \\
\hline $5: 00$ PM & 30 & 28 & 158 & 246 \\
\hline $6: 00$ PM & 132 & 68 & 710 & 65 \\
\hline $7: 00$ PM & 220 & 39 & 779 & 0 \\
\hline 8:00 PM & 213 & 200 & 245 & 154 \\
\hline $9: 00$ PM & 278 & 28 & 291 & 11 \\
\hline $10: 00$ PM & 271 & 59 & 312 & 21 \\
\hline $11: 00$ PM & 232 & 109 & 224 & 73 \\
\hline $12: 00$ AM & 211 & 59 & 211 & 46 \\
\hline $1: 00$ AM & 142 & 106 & 204 & 94 \\
\hline $2: 00$ AM & 665 & 40 & 517 & 2 \\
\hline $3: 00$ AM & 489 & 933 & 293 & 945 \\
\hline $4: 00$ AM & 224 & 14 & 100 & 5 \\
\hline $5: 00$ AM & 371 & 4 & 111 & 0 \\
\hline $6: 00$ AM & 333 & 0 & 109 & 0 \\
\hline $7: 00$ AM & 481 & 39 & 1505 & 22 \\
\hline $8: 00$ AM & 169 & 386 & 46 & 28 \\
\hline $9: 00$ AM & 137 & 322 & 23 & 153 \\
\hline $10: 00$ AM & 113 & 475 & 17 & 394 \\
\hline $11: 00$ AM & 173 & 318 & 370 & 242 \\
\hline $12: 00$ PM & 103 & 139 & 1432 & 82 \\
\hline $1: 00$ PM & 62 & 231 & 1127 & 56 \\
\hline $2: 00$ PM & 84 & 106 & 1120 & 203 \\
\hline \hline Total & 5151 & 3810 & 10415 & 2919 \\
\hline & & & & \\
\hline
\end{tabular}


Table 6-4: Hourly Discrepancy Totals for Phase 4, Blanking Band = 2 sec., February 23 \& 24, 2001, Rain

\begin{tabular}{|r|r|r|r|r|}
\cline { 2 - 5 } \multicolumn{1}{c|}{} & \multicolumn{2}{c|}{ System 1 } & \multicolumn{2}{c|}{ System 2 } \\
\hline \hline Hour Beginning & L0V1 & \multicolumn{1}{c|}{ L1V0 } & \multicolumn{1}{c|}{ L0V1 } & L1V0 \\
\hline \hline 4:00 PM & 107 & 32 & 13 & 325 \\
\hline $5: 00$ PM & 74 & 31 & 19 & 94 \\
\hline $6: 00$ PM & 206 & 50 & 114 & 31 \\
\hline $7: 00$ PM & 465 & 64 & 328 & 37 \\
\hline 8:00 PM & 515 & 100 & 302 & 93 \\
\hline $9: 00$ PM & 490 & 103 & 307 & 57 \\
\hline $10: 00$ PM & 396 & 51 & 258 & 78 \\
\hline $11: 00$ PM & 514 & 50 & 260 & 81 \\
\hline $12: 00$ AM & 422 & 50 & 215 & 53 \\
\hline $1: 00$ AM & 372 & 45 & 221 & 47 \\
\hline $2: 00$ AM & 591 & 50 & 286 & 87 \\
\hline $3: 00$ AM & 521 & 47 & 249 & 72 \\
\hline $4: 00$ AM & 214 & 14 & 128 & 12 \\
\hline $5: 00$ AM & 85 & 12 & 56 & 19 \\
\hline $6: 00$ AM & 178 & 5 & 116 & 17 \\
\hline $7: 00$ AM & 316 & 18 & 175 & 32 \\
\hline $8: 00$ AM & 96 & 5 & 19 & 61 \\
\hline $9: 00$ AM & 112 & 15 & 20 & 42 \\
\hline $10: 00$ AM & 122 & 19 & 66 & 34 \\
\hline $11: 00$ AM & 165 & 15 & 68 & 177 \\
\hline $12: 00$ PM & 233 & 31 & 141 & 15 \\
\hline $1: 00$ PM & 193 & 16 & 64 & 6 \\
\hline $2: 00$ PM & 187 & 32 & 88 & 11 \\
\hline \hline Total & 6575 & 854 & 3514 & 1482 \\
\hline & & & & \\
\hline
\end{tabular}


Table 6-5: Hourly Discrepancy Totals for Phase 5, Blanking Band = 2 sec., February 23 \& 24, 2001, Rain

\begin{tabular}{|c|r|r|r|r|}
\cline { 2 - 5 } \multicolumn{1}{c|}{} & \multicolumn{2}{c|}{ System 1 } & \multicolumn{2}{c|}{ System 2 } \\
\hline Hour Beginning & L0V1 & L1V0 & L0V1 & L1V0 \\
\hline \hline 4:00 PM & 75 & 88 & 0 & 64 \\
\hline $5: 00$ PM & 0 & 71 & 2 & 60 \\
\hline $6: 00$ PM & 20 & 20 & 160 & 56 \\
\hline $7: 00$ PM & 91 & 98 & 52 & 62 \\
\hline 8:00 PM & 100 & 99 & 54 & 76 \\
\hline $9: 00$ PM & 76 & 97 & 54 & 58 \\
\hline $10: 00$ PM & 109 & 150 & 39 & 129 \\
\hline $11: 00$ PM & 91 & 69 & 39 & 52 \\
\hline $12: 00$ AM & 146 & 86 & 27 & 80 \\
\hline $1: 00$ AM & 103 & 16 & 20 & 47 \\
\hline $2: 00$ AM & 110 & 99 & 47 & 85 \\
\hline $3: 00$ AM & 66 & 111 & 26 & 115 \\
\hline $4: 00$ AM & 42 & 6 & 34 & 3 \\
\hline $5: 00$ AM & 57 & 2 & 20 & 0 \\
\hline $6: 00$ AM & 160 & 61 & 44 & 0 \\
\hline $7: 00$ AM & 232 & 114 & 1506 & 34 \\
\hline $8: 00$ AM & 123 & 77 & 5 & 4 \\
\hline $9: 00$ AM & 8 & 183 & 0 & 25 \\
\hline $10: 00$ AM & 3 & 136 & 17 & 31 \\
\hline $11: 00$ AM & 6 & 109 & 54 & 23 \\
\hline $12: 00$ PM & 0 & 61 & 0 & 60 \\
\hline $1: 00$ PM & 0 & 9 & 0 & 11 \\
\hline $2: 00$ PM & 0 & 31 & 0 & 26 \\
\hline \hline Total & 1618 & 1791 & 2201 & 1099 \\
\hline & & & & \\
\hline \hline
\end{tabular}


Table 6-6: Hourly Discrepancy Totals for Phase 6, Blanking Band = 2 sec., February 23 \& 24, 2001, Rain

\begin{tabular}{|c|r|r|r|r|}
\cline { 2 - 5 } \multicolumn{1}{c|}{} & \multicolumn{2}{c|}{ System 1 } & \multicolumn{2}{c|}{ System 2 } \\
\hline Hour Beginning & \multicolumn{1}{c|}{ L0V1 } & L1V0 & LOV1 & L1V0 \\
\hline \hline $4: 00$ PM & 10 & 4 & 5 & 424 \\
\hline $5: 00$ PM & 16 & 4 & 7 & 697 \\
\hline $6: 00$ PM & 126 & 5 & 76 & 657 \\
\hline $7: 00$ PM & 226 & 15 & 144 & 371 \\
\hline $8: 00$ PM & 283 & 9 & 155 & 583 \\
\hline $9: 00$ PM & 254 & 170 & 149 & 639 \\
\hline $10: 00$ PM & 271 & 2 & 132 & 450 \\
\hline $11: 00$ PM & 260 & 22 & 137 & 474 \\
\hline $12: 00$ AM & 229 & 15 & 105 & 555 \\
\hline $1: 00$ AM & 172 & 0 & 106 & 238 \\
\hline $2: 00$ AM & 456 & 22 & 142 & 119 \\
\hline $3: 00$ AM & 345 & 52 & 102 & 245 \\
\hline $4: 00$ AM & 179 & 0 & 28 & 58 \\
\hline $5: 00$ AM & 204 & 2 & 35 & 10 \\
\hline $6: 00$ AM & 275 & 0 & 120 & 37 \\
\hline $7: 00$ AM & 277 & 19 & 148 & 274 \\
\hline $8: 00$ AM & 67 & 5 & 33 & 532 \\
\hline $9: 00$ AM & 10 & 7 & 7 & 607 \\
\hline $10: 00$ AM & 24 & 3 & 9 & 691 \\
\hline $11: 00$ AM & 81 & 53 & 44 & 657 \\
\hline $12: 00$ PM & 54 & 0 & 41 & 782 \\
\hline $1: 00$ PM & 48 & 4 & 8 & 791 \\
\hline $2: 00$ PM & 41 & 0 & 18 & 884 \\
\hline Total & 3909 & 411 & 1749 & 10776 \\
\hline
\end{tabular}


Table 6-7: Hourly Discrepancy Totals for Phase 7, Blanking Band = 2 sec., February 23 \& 24, 2001, Rain

\begin{tabular}{|c|r|r|r|r|}
\cline { 2 - 5 } \multicolumn{1}{c|}{} & \multicolumn{2}{c|}{ System 1 } & \multicolumn{2}{c|}{ System 2 } \\
\hline Hour Beginning & L0V1 & L1V0 & L0V1 & L1V0 \\
\hline \hline 4:00 PM & 293 & 69 & 485 & 134 \\
\hline $5: 00$ PM & 128 & 69 & 1020 & 226 \\
\hline $6: 00$ PM & 101 & 0 & 3244 & 3 \\
\hline $7: 00$ PM & 146 & 28 & 1698 & 49 \\
\hline 8:00 PM & 102 & 14 & 123 & 46 \\
\hline $9: 00$ PM & 40 & 6 & 107 & 6 \\
\hline $10: 00$ PM & 84 & 0 & 77 & 6 \\
\hline $11: 00$ PM & 57 & 5 & 122 & 8 \\
\hline $12: 00$ AM & 43 & 2 & 46 & 16 \\
\hline $1: 00$ AM & 40 & 14 & 2 & 42 \\
\hline $2: 00$ AM & 265 & 0 & 141 & 0 \\
\hline $3: 00$ AM & 410 & 0 & 21 & 0 \\
\hline $4: 00$ AM & 68 & 0 & 27 & 0 \\
\hline $5: 00$ AM & 7 & 0 & 11 & 0 \\
\hline $6: 00$ AM & 11 & 0 & 5 & 0 \\
\hline $7: 00$ AM & 73 & 0 & 1944 & 0 \\
\hline $8: 00$ AM & 22 & 32 & 1017 & 43 \\
\hline $9: 00$ AM & 17 & 0 & 9 & 2 \\
\hline $10: 00$ AM & 78 & 38 & 24 & 0 \\
\hline $11: 00$ AM & 187 & 22 & 796 & 29 \\
\hline $12: 00$ PM & 95 & 0 & 140 & 30 \\
\hline $1: 00$ PM & 82 & 173 & 185 & 13 \\
\hline $2: 00$ PM & 46 & 30 & 73 & 26 \\
\hline \hline Total & 2396 & 501 & 11316 & 678 \\
\hline
\end{tabular}


Table 6-8: Hourly Discrepancy Totals for Phase 8, Blanking Band = 2 sec., February 23 \& 24, 2001, Rain

\begin{tabular}{|c|c|c|c|c|}
\hline & \multicolumn{2}{|c|}{ System 1} & \multicolumn{2}{|c|}{ System 2} \\
\hline Hour Beginning & LOV1 & L1V0 & LoV1 & L1V0 \\
\hline 4:00 PM & 9 & 49 & 9 & 55 \\
\hline 5:00 PM & 9 & 45 & 0 & 46 \\
\hline 6:00 PM & 115 & 126 & 72 & 99 \\
\hline 7:00 PM & 322 & 102 & 300 & 90 \\
\hline 8:00 PM & 355 & 161 & 312 & 152 \\
\hline 9:00 PM & 387 & 251 & 330 & 221 \\
\hline 10:00 PM & 427 & 5 & 373 & 0 \\
\hline 11:00 PM & 490 & 45 & 379 & 22 \\
\hline 12:00 AM & 427 & 126 & 337 & 84 \\
\hline 1:00 AM & 486 & 48 & 390 & 28 \\
\hline 2:00 AM & 1184 & 5 & 633 & 3 \\
\hline 3:00 AM & 1116 & 29 & 419 & 3 \\
\hline 4:00 AM & 266 & 123 & 163 & 139 \\
\hline 5:00 AM & 257 & 64 & 153 & 69 \\
\hline 6:00 AM & 254 & 5 & 151 & 0 \\
\hline 7:00 AM & 590 & 36 & 317 & 14 \\
\hline 8:00 AM & 83 & 86 & 55 & 58 \\
\hline 9:00 AM & 13 & 341 & 0 & 339 \\
\hline 10:00 AM & 23 & 229 & 19 & 219 \\
\hline $11: 00 \mathrm{AM}$ & 77 & 168 & 58 & 162 \\
\hline 12:00 PM & 56 & 225 & 43 & 204 \\
\hline 1:00 PM & 25 & 46 & 12 & 38 \\
\hline $2: 00 \mathrm{PM}$ & 31 & 94 & 33 & 83 \\
\hline Total & 7003 & 2406 & 4558 & 2129 \\
\hline
\end{tabular}

One can see a very large spike in Figure 6-1c. This spike is due to the video detector continuously registering a call, even though no vehicles are present. This type of error is not uncommon, and can also be seen in Figure 6-7c. It appears that during this data collection, System 1 had less of a tendency to stick on than did System 2.

As previously mentioned, it began to rain at approximately 2:00 AM. As can be seen in several of the figures corresponding to this data collection, the LOV1 errors became slightly larger at this time due the glare effect that rain has on the pavement. The rain causes the pavement to reflect headlights even more 
than normal, thus causing the headlights to reach the detection zone earlier than usual. For this reason, rain at night can cause video detection systems to operate even less efficiently than when it is not raining.

\section{Overcast Conditions (Before Recalibration)}

From 1:00 PM on March 4 - 1:00 PM on March 5, 2001, another data collection was performed. On these days, the sky was overcast, or the sun was not out. This data collection was performed because overcast conditions have been determined to be optimal for video detection systems because there are no shadows during the day. It can be argued that the overcast conditions are not optimal for the night, because the moonlight could help the video detectors to better distinguish vehicles. This may be true, but the overcast conditions are advantageous for the daytime.

Figure 6-9 through Figure 6-16 show the LOV1 and L1V0 discrepancies for both video detection systems for all eight phases. Table 6-9 through Table 6-16 gives the hourly discrepancy totals, in seconds, for each of the phases, as well. It is obvious that there is a problem with the System 2 detector on phase 6 . The L1V0 errors on this phase are very large. This is because the detector was not detecting vehicles most of the time. The reason for which the discrepancies are higher during the day is the larger queue delays due to the higher traffic volume on all approaches. 


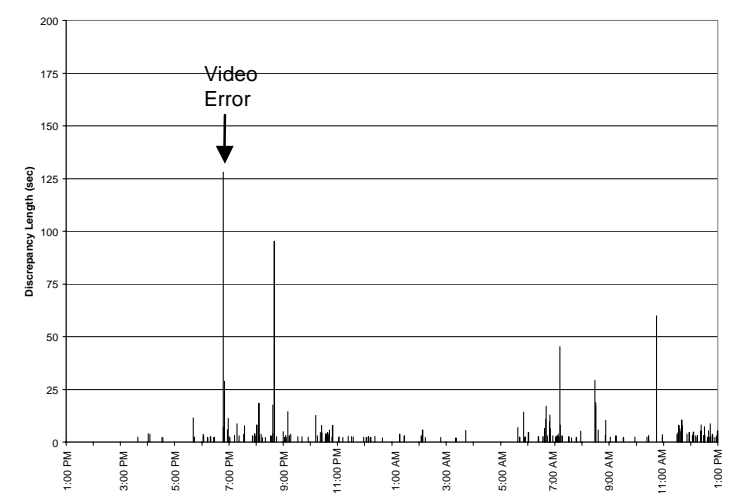

a) System 1 LOV1

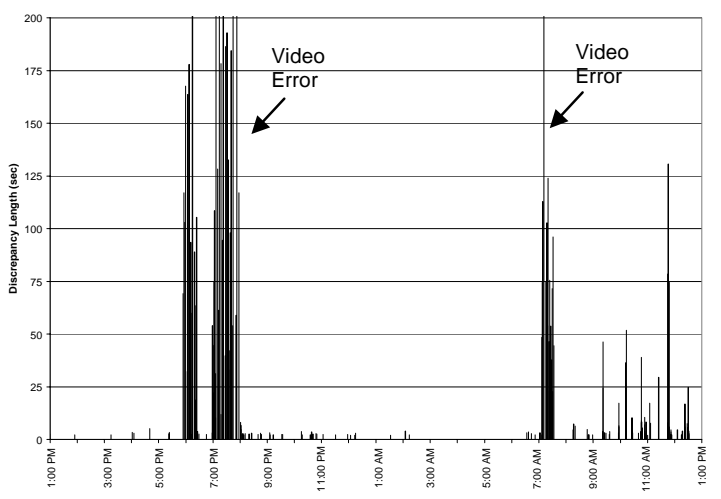

c) System 2 LOV1

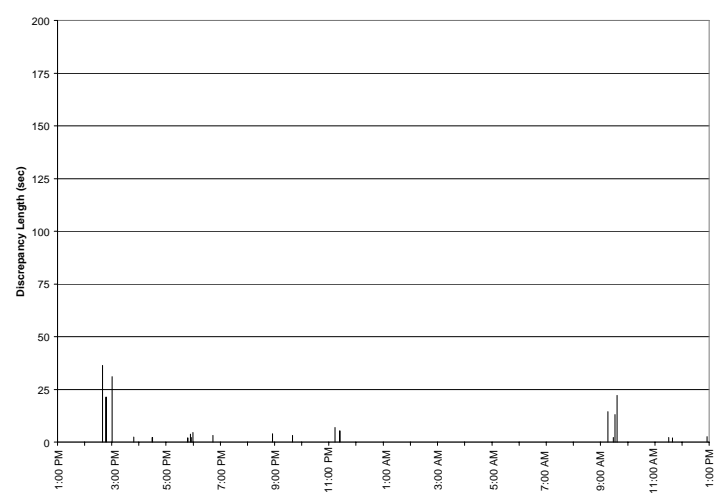

b) System $1 \mathrm{~L} 1 \mathrm{~V} 0$

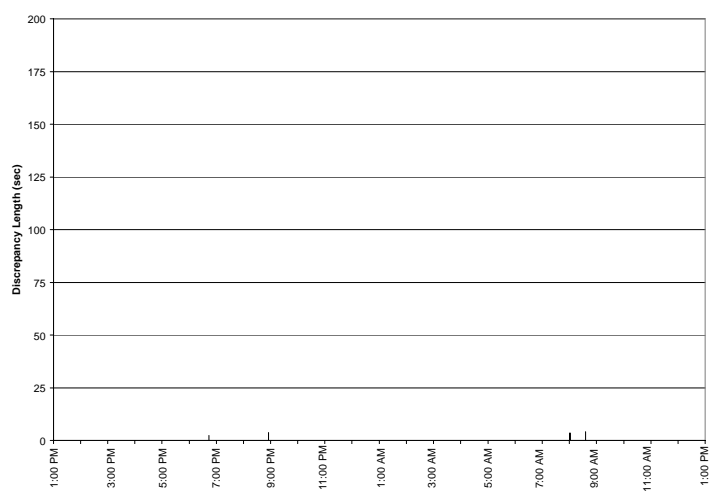

d) System 2 L1V0

Figure 6-9: Phase 1, Blanking Band $=2$ sec., March 4 \& 5, 2001, Overcast 


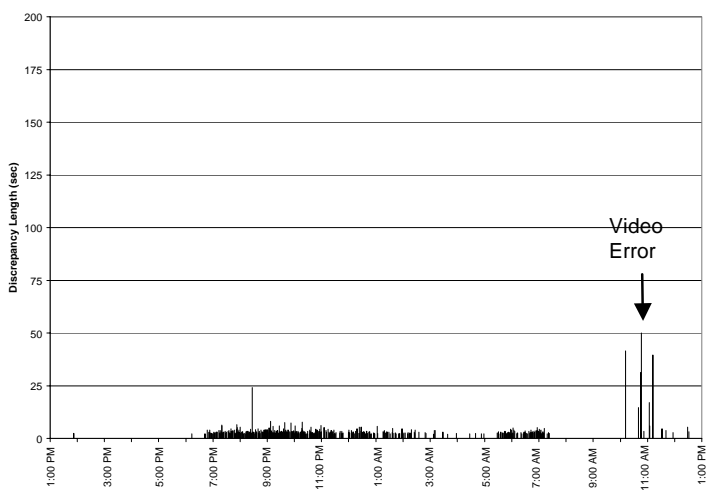

a) System 1 LOV1

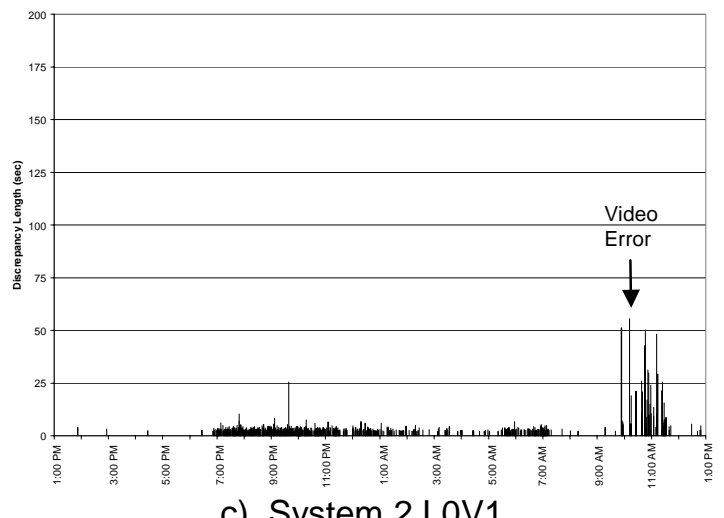

c) System 2 LOV1

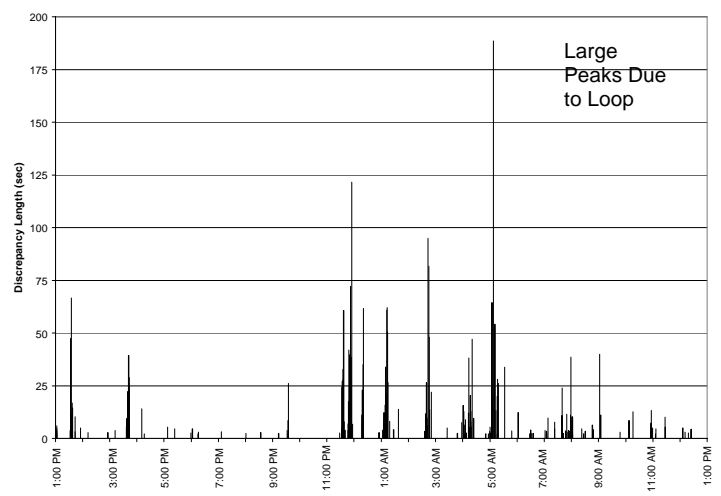

b) System 1 L1V0

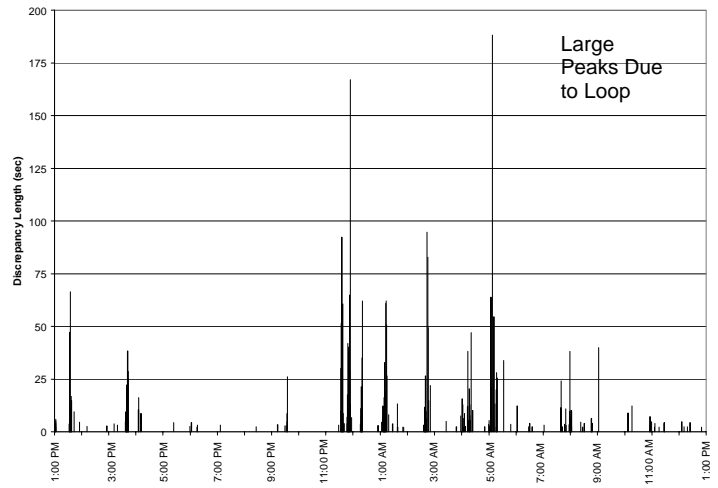

d) System 2 L1V0

Figure 6-10: Phase 2, Blanking Band = 2 sec., March $4 \&$ 5, 2001, Overcast 


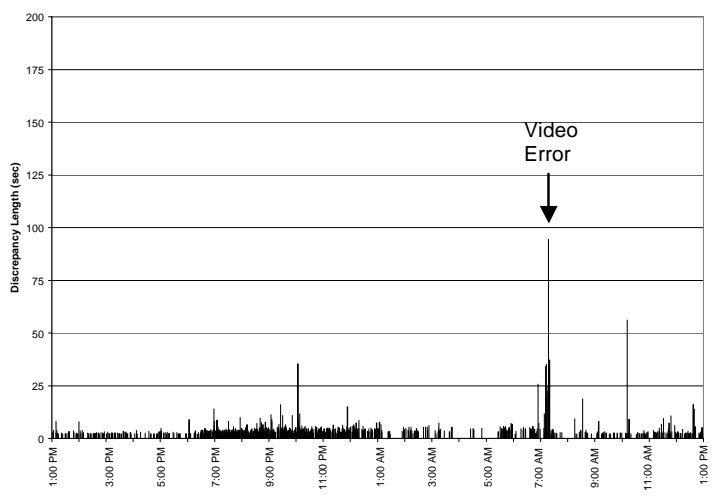

a) System 1 LOV1

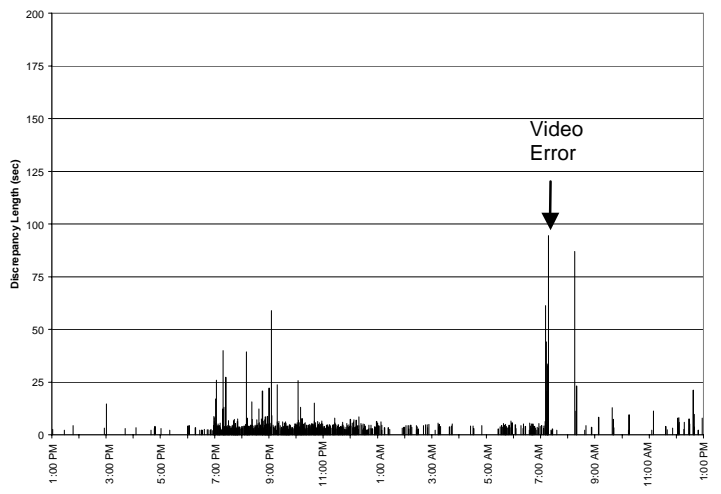

c) System 2 LOV1

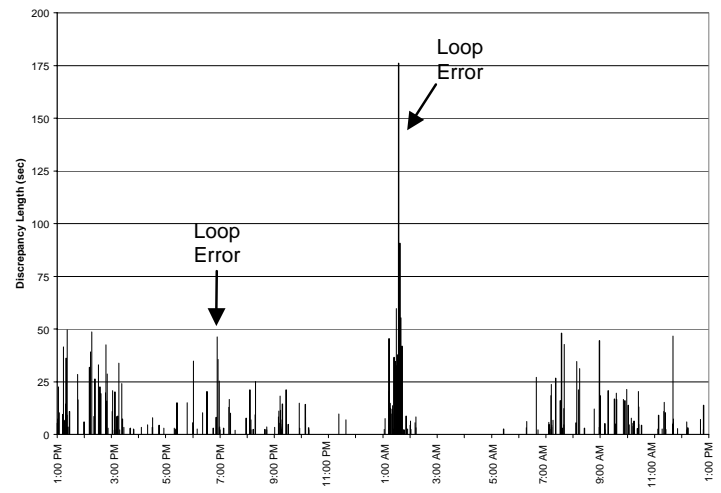

b) System 1 L1V0

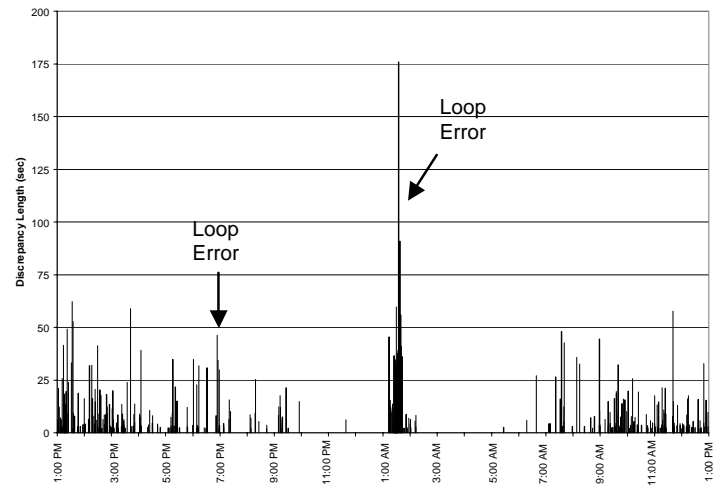

d) System 2 L1V0

Figure 6-11: Phase 3, Blanking Band = 2 sec., March 4 \& 5, 2001, Overcast 


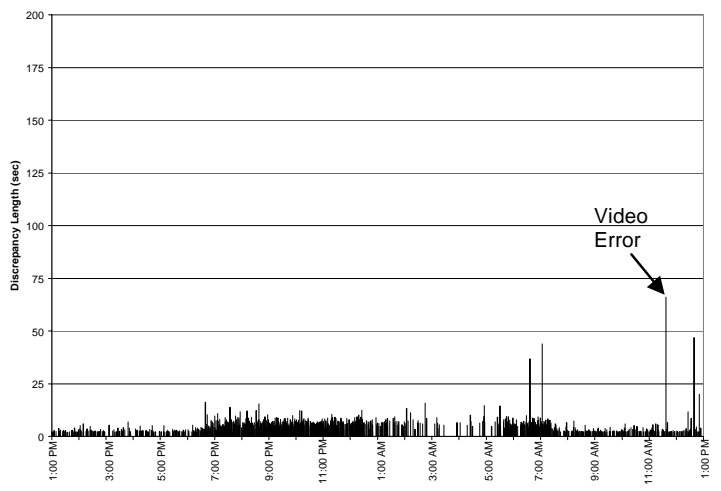

a) System 1 LOV1

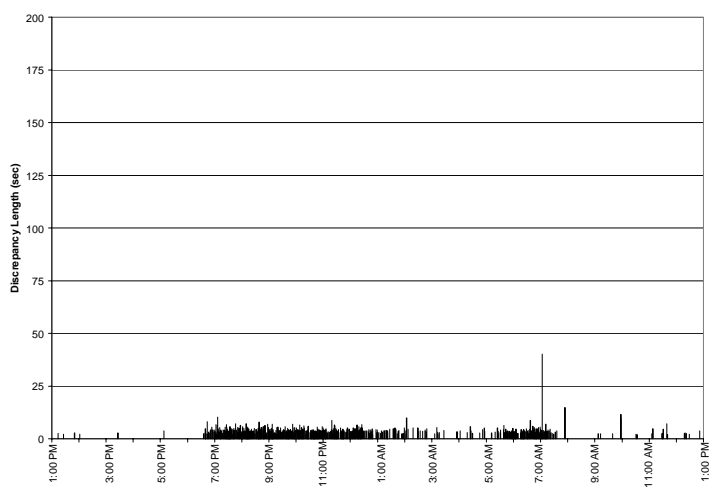

c) System 2 LOV1

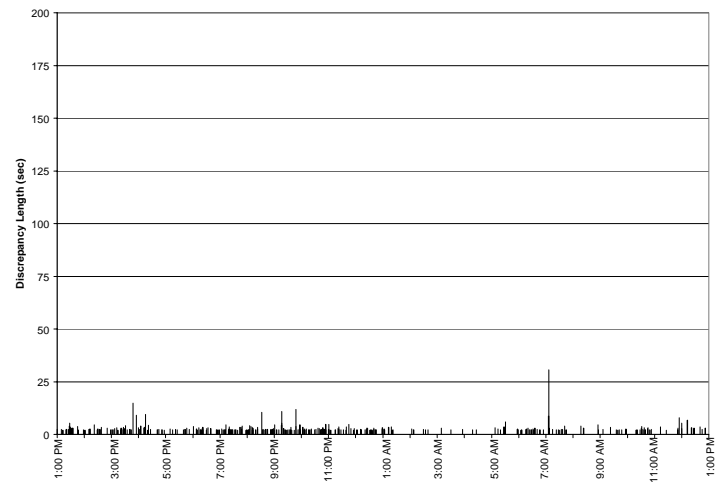

b) System 1 L1V0

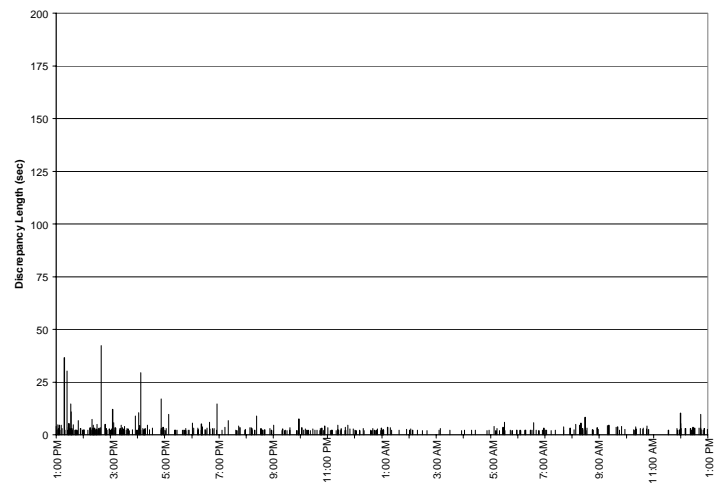

d) System 2 L1V0

Figure 6-12: Phase 4, Blanking Band = 2 sec., March 4 \& 5, 2001 Overcast 


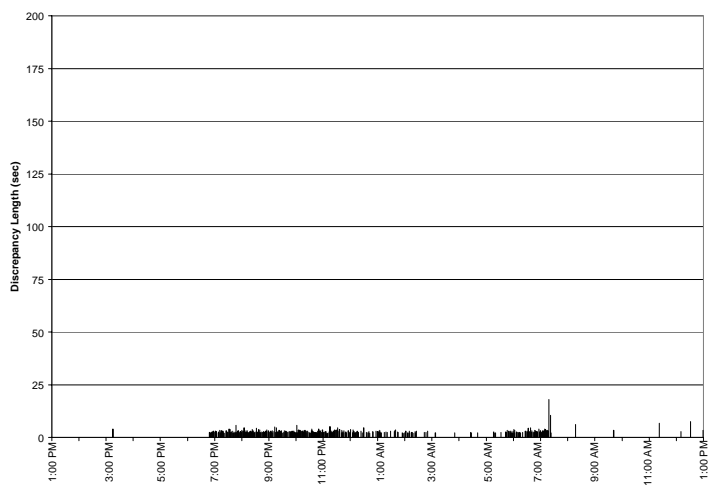

a) System 1 LOV1

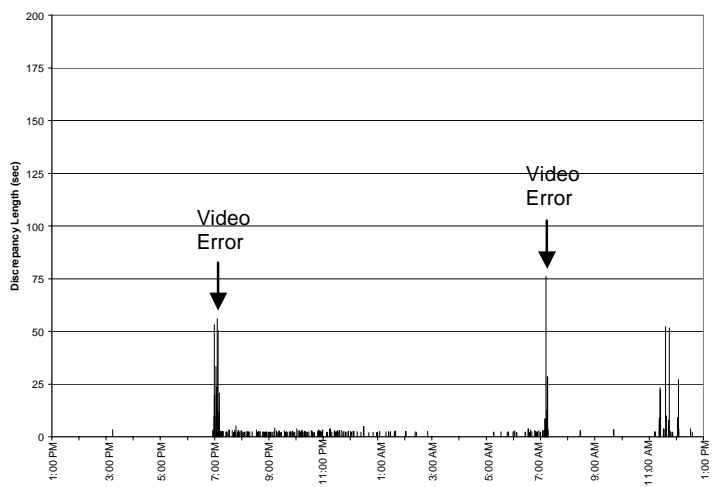

c) System 2 LOV1

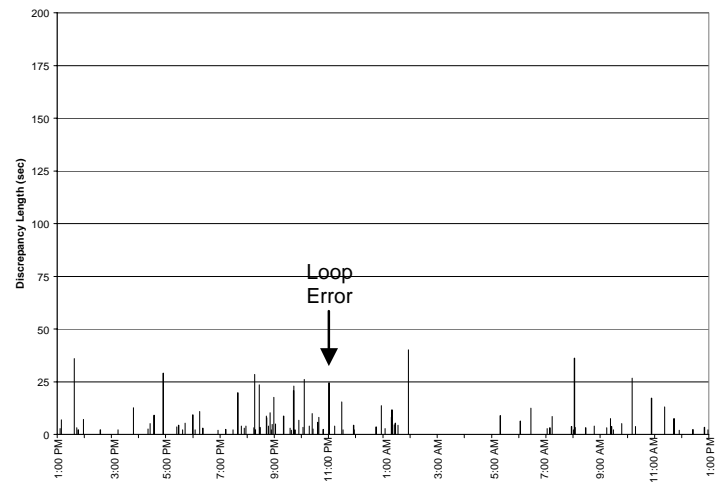

b) System 1 L1V0

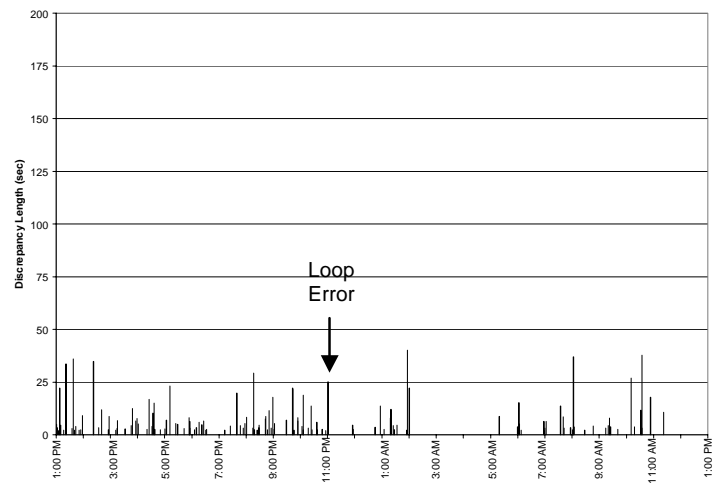

d) System 2 L1V0

Figure 6-13: Phase 5, Blanking Band = 2 sec., March 4 \& 5, 2001 Overcast 


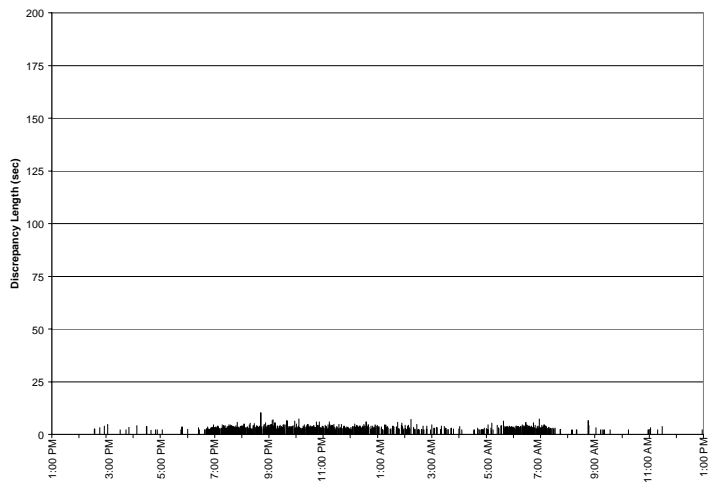

a) System 1 LOV1

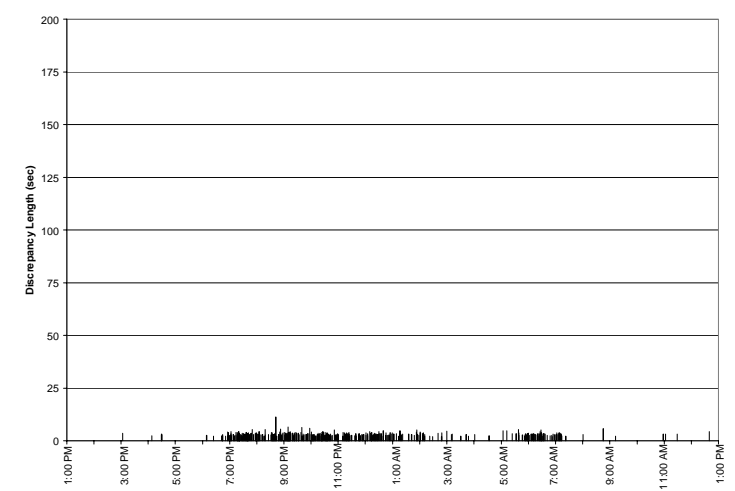

c) System 2 LOV1

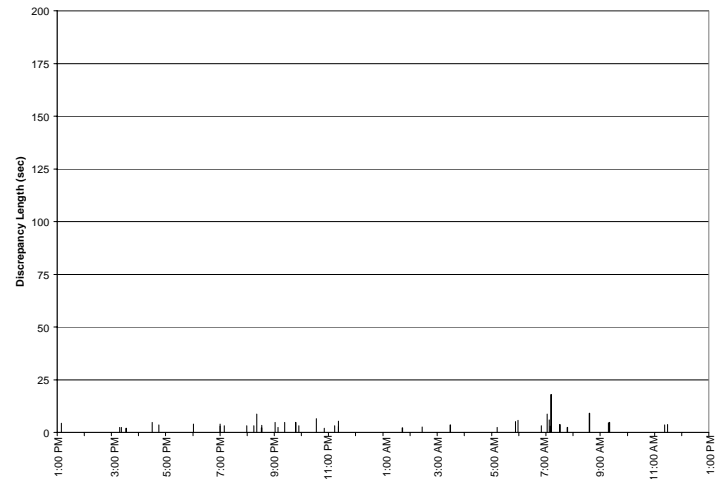

b) System 1 L1V0

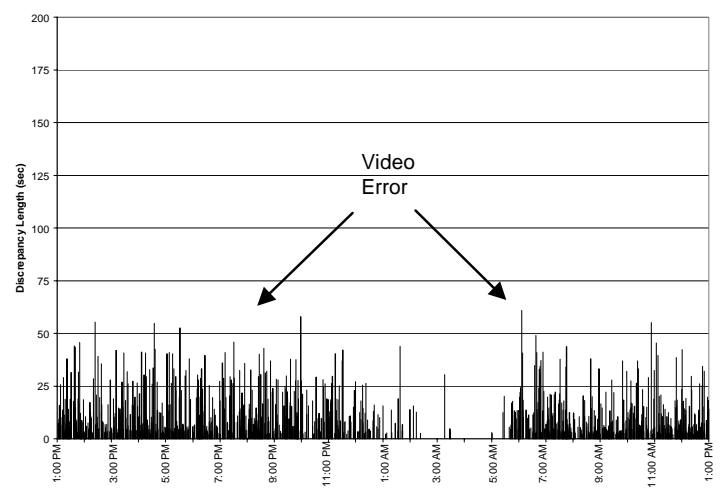

d) System 2 L1V0

Figure 6-14: Phase 6, Blanking Band $=2$ sec., March 4 \& 5, 2001 Overcast 


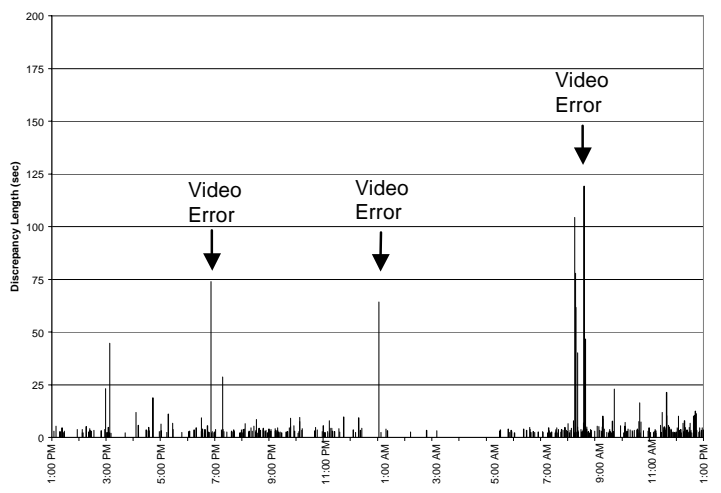

a) System 1 LOV1

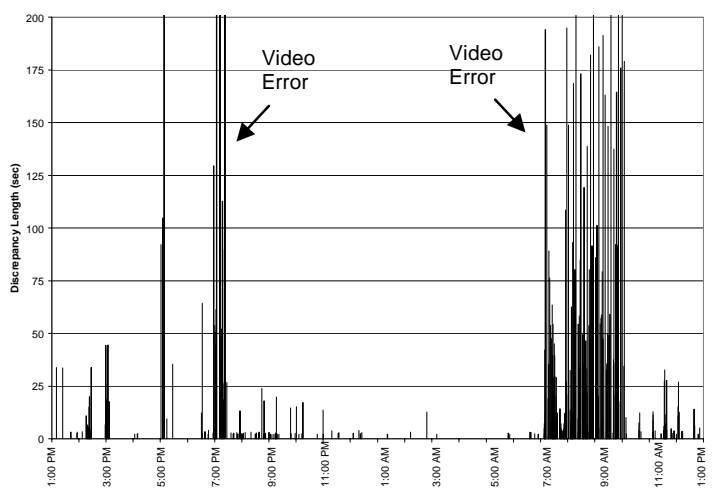

c) System 2 LOV1

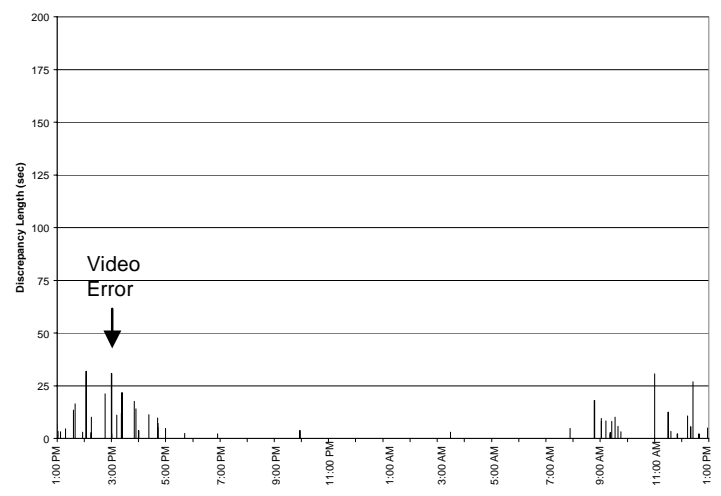

b) System 1 L1V0

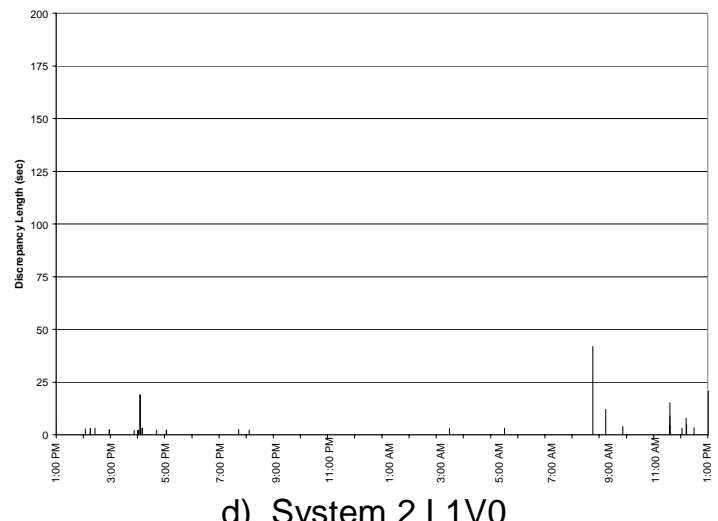

d) System 2 L1V0

Figure 6-15: Phase 7, Blanking Band = 2 sec., March 4 \& 5, 2001 Overcast 


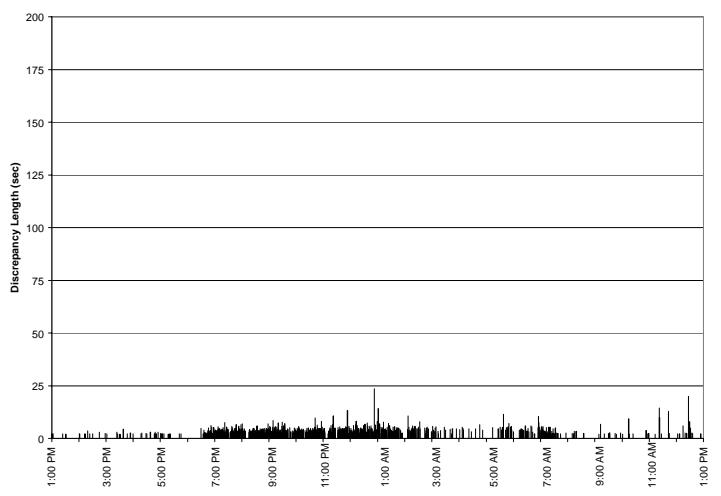

b) System 1 LOV1

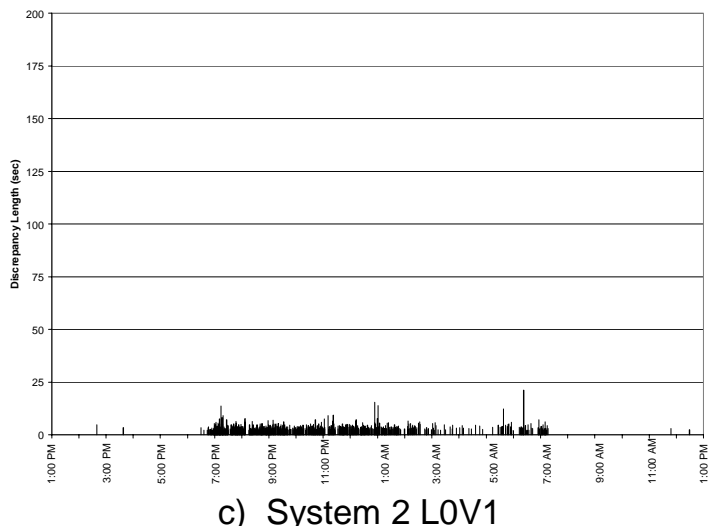

c) System 2 LOV1

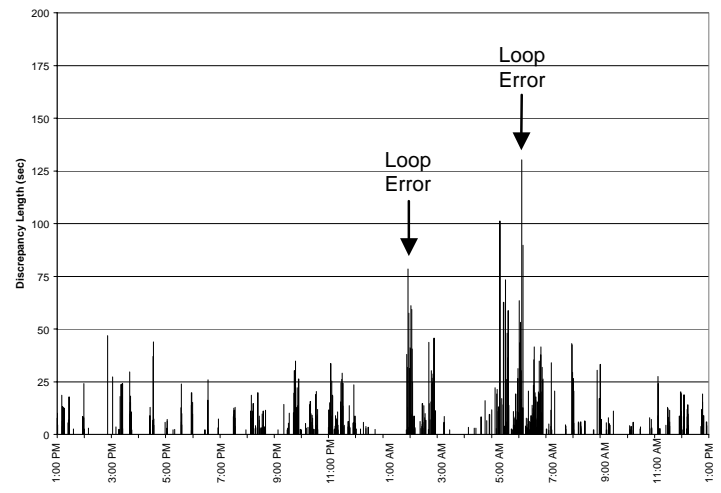

b) System 1 L1V0

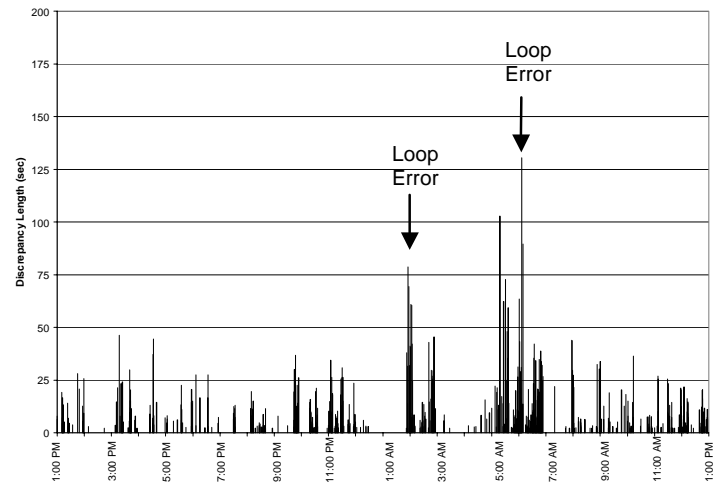

d) System 2 L1V0

Figure 6-16: Phase 8, Blanking Band = 2 sec., March 4 \& 5, 2001 Overcast 
Table 6-9: Hourly Discrepancy Totals for Phase 1, Blanking Band = $2 \mathrm{sec}$, March 4 \& 5, 2001, Overcast

\begin{tabular}{|c|r|r|r|r|}
\cline { 2 - 5 } \multicolumn{1}{c|}{} & \multicolumn{2}{c|}{ System 1 } & \multicolumn{2}{c|}{ System 2 } \\
\hline \hline Hour Beginning & \multicolumn{1}{c|}{ L0V1 } & \multicolumn{1}{c|}{ L1V0 } & L0V1 & \multicolumn{1}{c|}{ L1V0 } \\
\hline \hline 1:00 PM & 0 & 0 & 2 & 0 \\
\hline $2: 00$ PM & 0 & 58 & 0 & 0 \\
\hline $3: 00$ PM & 2 & 34 & 2 & 0 \\
\hline $4: 00$ PM & 12 & 2 & 11 & 0 \\
\hline $5: 00$ PM & 14 & 12 & 463 & 0 \\
\hline $6: 00$ PM & 201 & 3 & 1206 & 2 \\
\hline $7: 00$ PM & 43 & 0 & 3108 & 0 \\
\hline $8: 00$ PM & 160 & 4 & 52 & 4 \\
\hline $9: 00$ PM & 43 & 3 & 12 & 0 \\
\hline $10: 00$ PM & 70 & 0 & 20 & 0 \\
\hline $11: 00$ PM & 17 & 12 & 7 & 0 \\
\hline $12: 00$ AM & 14 & 0 & 7 & 0 \\
\hline $1: 00$ AM & 7 & 0 & 2 & 0 \\
\hline $2: 00$ AM & 13 & 0 & 6 & 0 \\
\hline $3: 00$ AM & 8 & 0 & 0 & 0 \\
\hline $4: 00$ AM & 0 & 0 & 0 & 0 \\
\hline $5: 00$ AM & 30 & 0 & 0 & 0 \\
\hline $6: 00$ AM & 91 & 0 & 12 & 0 \\
\hline $7: 00$ AM & 83 & 0 & 1454 & 0 \\
\hline $8: 00$ AM & 84 & 0 & 34 & 8 \\
\hline $9: 00$ AM & 10 & 52 & 120 & 0 \\
\hline $10: 00$ AM & 73 & 0 & 295 & 0 \\
\hline $11: 00$ AM & 66 & 4 & 376 & 0 \\
\hline $12: 00$ PM & 99 & 3 & 96 & 0 \\
\hline \hline Total & 1138 & 187 & 7284 & 14 \\
\hline
\end{tabular}


Table 6-10: Hourly Discrepancy Totals for Phase 2, Blanking Band = $2 \mathrm{sec}$, March 4 \& 5, 2001, Overcast

\begin{tabular}{|c|r|r|r|r|}
\cline { 2 - 5 } \multicolumn{1}{c|}{} & \multicolumn{2}{c|}{ System 1 } & \multicolumn{2}{c|}{ System 2 } \\
\hline Hour Beginning & L0V1 & L1V0 & L0V1 & L1V0 \\
\hline \hline 1:00 PM & 2 & 227 & 4 & 223 \\
\hline $2: 00 \mathrm{PM}$ & 0 & 5 & 3 & 5 \\
\hline $3: 00 \mathrm{PM}$ & 0 & 209 & 0 & 207 \\
\hline $4: 00 \mathrm{PM}$ & 0 & 16 & 2 & 39 \\
\hline $5: 00 \mathrm{PM}$ & 0 & 13 & 0 & 7 \\
\hline $6: 00 \mathrm{PM}$ & 22 & 10 & 16 & 10 \\
\hline $7: 00 \mathrm{PM}$ & 159 & 3 & 215 & 3 \\
\hline 8:00 PM & 178 & 5 & 195 & 2 \\
\hline 9:00 PM & 226 & 43 & 237 & 49 \\
\hline $10: 00 \mathrm{PM}$ & 192 & 0 & 211 & 0 \\
\hline $11: 00 \mathrm{PM}$ & 93 & 744 & 119 & 755 \\
\hline $12: 00 \mathrm{AM}$ & 108 & 181 & 114 & 185 \\
\hline $1: 00 \mathrm{AM}$ & 63 & 401 & 64 & 402 \\
\hline $2: 00 \mathrm{AM}$ & 40 & 348 & 36 & 356 \\
\hline $3: 00 \mathrm{AM}$ & 17 & 15 & 27 & 15 \\
\hline $4: 00 \mathrm{AM}$ & 9 & 226 & 19 & 224 \\
\hline $5: 00 \mathrm{AM}$ & 53 & 457 & 70 & 457 \\
\hline $6: 00 \mathrm{AM}$ & 103 & 23 & 112 & 21 \\
\hline $7: 00 \mathrm{AM}$ & 50 & 160 & 60 & 120 \\
\hline 8:00 AM & 0 & 70 & 4 & 70 \\
\hline 9:00 AM & 0 & 54 & 86 & 40 \\
\hline $10: 00 \mathrm{AM}$ & 143 & 62 & 458 & 43 \\
\hline $11: 00 \mathrm{AM}$ & 74 & 22 & 240 & 17 \\
\hline $12: 00 \mathrm{PM}$ & 9 & 15 & 17 & 16 \\
\hline \hline Total & 1541 & 3307 & 2313 & 3264 \\
\hline & & & & \\
\hline
\end{tabular}


Table 6-11: Hourly Discrepancy Totals for Phase 3, Blanking Band = 2 sec., March 4 \& 5, 2001, Overcast

\begin{tabular}{|c|c|c|c|c|}
\hline & \multicolumn{2}{|c|}{ System 1} & \multicolumn{2}{|c|}{ System 2} \\
\hline Hour Beginning & L0V1 & L1V0 & LoV1 & L1V0 \\
\hline 1:00 PM & 70 & 296 & 9 & 518 \\
\hline 2:00 PM & 49 & 344 & 3 & 372 \\
\hline $3: 00 \mathrm{PM}$ & 60 & 157 & 17 & 235 \\
\hline 4:00 PM & 36 & 26 & 13 & 109 \\
\hline $5: 00$ PM & 45 & 48 & 5 & 148 \\
\hline 6:00 PM & 157 & 204 & 70 & 265 \\
\hline 7:00 PM & 294 & 54 & 430 & 39 \\
\hline 8:00 PM & 272 & 77 & 406 & 63 \\
\hline 9:00 PM & 286 & 138 & 354 & 109 \\
\hline 10:00 PM & 290 & 36 & 352 & 0 \\
\hline 11:00 PM & 231 & 17 & 230 & 6 \\
\hline $12: 00 \mathrm{AM}$ & 178 & 0 & 162 & 0 \\
\hline $1: 00 \mathrm{AM}$ & 67 & 1042 & 54 & 1043 \\
\hline $2: 00 \mathrm{AM}$ & 83 & 30 & 67 & 29 \\
\hline $3: 00 \mathrm{AM}$ & 46 & 0 & 33 & 0 \\
\hline $4: 00 \mathrm{AM}$ & 18 & 0 & 16 & 0 \\
\hline 5:00 AM & 88 & 3 & 73 & 3 \\
\hline $6: 00$ AM & 127 & 38 & 95 & 33 \\
\hline 7:00 AM & 341 & 236 & 397 & 179 \\
\hline $8: 00 \mathrm{AM}$ & 59 & 164 & 131 & 153 \\
\hline 9:00 AM & 49 & 159 & 35 & 237 \\
\hline 10:00 AM & 116 & 89 & 12 & 144 \\
\hline $11: 00 \mathrm{AM}$ & 115 & 132 & 23 & 306 \\
\hline 12:00 PM & 140 & 38 & 109 & 234 \\
\hline Total & 3216 & 3328 & 3096 & 4225 \\
\hline
\end{tabular}


Table 6-12: Hourly Discrepancy Totals for Phase 4, Blanking Band = 2 sec., March 4 \& 5, 2001, Overcast

\begin{tabular}{|c|r|r|r|r|}
\cline { 2 - 5 } \multicolumn{1}{c|}{} & \multicolumn{2}{c|}{ System 1 } & \multicolumn{2}{c|}{ System } \\
\hline \hline Hour Beginning & \multicolumn{1}{c|}{ L0V1 } & L0V1 & \multicolumn{1}{c|}{ L1V0 } \\
\hline \hline 1:00 PM & 61 & 43 & 8 & 183 \\
\hline $2: 00$ PM & 97 & 29 & 2 & 134 \\
\hline $3: 00$ PM & 75 & 70 & 3 & 84 \\
\hline $4: 00$ PM & 66 & 51 & 0 & 115 \\
\hline $5: 00$ PM & 67 & 21 & 4 & 38 \\
\hline $6: 00$ PM & 178 & 44 & 69 & 79 \\
\hline $7: 00$ PM & 380 & 70 & 262 & 32 \\
\hline 8:00 PM & 417 & 71 & 266 & 42 \\
\hline $9: 00$ PM & 431 & 83 & 263 & 34 \\
\hline $10: 00$ PM & 466 & 66 & 290 & 44 \\
\hline $11: 00$ PM & 388 & 46 & 227 & 48 \\
\hline $12: 00$ AM & 308 & 44 & 176 & 38 \\
\hline $1: 00$ AM & 168 & 20 & 91 & 22 \\
\hline $2: 00$ AM & 110 & 11 & 57 & 13 \\
\hline $3: 00$ AM & 42 & 8 & 24 & 9 \\
\hline $4: 00$ AM & 86 & 4 & 39 & 10 \\
\hline $5: 00$ AM & 176 & 26 & 79 & 31 \\
\hline $6: 00$ AM & 385 & 35 & 203 & 30 \\
\hline $7: 00$ AM & 270 & 64 & 142 & 18 \\
\hline 8:00 AM & 109 & 14 & 0 & 52 \\
\hline $9: 00$ AM & 86 & 23 & 40 & 25 \\
\hline $10: 00$ AM & 104 & 35 & 4 & 33 \\
\hline $11: 00$ AM & 177 & 21 & 26 & 22 \\
\hline $12: 00$ PM & 166 & 30 & 16 & 54 \\
\hline \hline Total & 4815 & 929 & 2290 & 1190 \\
\hline
\end{tabular}


Table 6-13: Hourly Discrepancy Totals for Phase 5, Blanking Band = 2 sec., March 4 \& 5, 2001, Overcast

\begin{tabular}{|c|c|c|c|c|}
\hline & \multicolumn{2}{|c|}{ System 1} & \multicolumn{2}{|c|}{ System 2} \\
\hline Hour Beginning & L0V1 & L1V0 & LoV1 & L1V0 \\
\hline 1:00 PM & 0 & 58 & 0 & 131 \\
\hline 2:00 PM & 0 & 2 & 0 & 61 \\
\hline 3:00 PM & 4 & 15 & 3 & 46 \\
\hline 4:00 PM & 0 & 46 & 0 & 59 \\
\hline 5:00 PM & 0 & 25 & 0 & 64 \\
\hline 6:00 PM & 15 & 18 & 88 & 28 \\
\hline 7:00 PM & 111 & 35 & 293 & 39 \\
\hline 8:00 PM & 140 & 116 & 49 & 104 \\
\hline 9:00 PM & 127 & 71 & 53 & 75 \\
\hline 10:00 PM & 121 & 62 & 60 & 59 \\
\hline 11:00 PM & 111 & 53 & 42 & 32 \\
\hline 12:00 AM & 68 & 17 & 27 & 17 \\
\hline $1: 00 \mathrm{AM}$ & 47 & 77 & 17 & 76 \\
\hline $2: 00$ AM & 35 & 0 & 10 & 22 \\
\hline $3: 00 \mathrm{AM}$ & 4 & 0 & 0 & 0 \\
\hline 4:00 AM & 7 & 0 & 0 & 0 \\
\hline 5:00 AM & 40 & 9 & 11 & 13 \\
\hline 6:00 AM & 87 & 19 & 38 & 31 \\
\hline 7:00 AM & 90 & 18 & 180 & 38 \\
\hline $8: 00 \mathrm{AM}$ & 6 & 49 & 3 & 49 \\
\hline 9:00 AM & 3 & 27 & 3 & 28 \\
\hline 10:00 AM & 0 & 48 & 0 & 101 \\
\hline $11: 00 \mathrm{AM}$ & 7 & 23 & 222 & 11 \\
\hline 12:00 PM & 13 & 10 & 57 & 0 \\
\hline Total & 1037 & 797 & 1159 & 1083 \\
\hline
\end{tabular}


Table 6-14: Hourly Discrepancy Totals for Phase 6, Blanking Band $=2 \mathrm{sec}$., March 4 \& 5, 2001, Overcast

\begin{tabular}{|c|r|r|r|r|}
\cline { 2 - 5 } \multicolumn{1}{c|}{} & \multicolumn{2}{c|}{ System 1 } & \multicolumn{2}{c|}{ System 2 } \\
\hline \hline Hour Beginning & \multicolumn{1}{c|}{ L0V1 } & L1V0 & L0V1 & L1V0 \\
\hline \hline 1:00 PM & 0 & 4 & 0 & 764 \\
\hline $2: 00$ PM & 12 & 0 & 0 & 551 \\
\hline $3: 00$ PM & 12 & 7 & 4 & 625 \\
\hline $4: 00$ PM & 14 & 8 & 8 & 696 \\
\hline $5: 00$ PM & 8 & 0 & 0 & 676 \\
\hline $6: 00$ PM & 65 & 4 & 30 & 588 \\
\hline $7: 00$ PM & 268 & 14 & 178 & 676 \\
\hline $8: 00$ PM & 248 & 18 & 132 & 693 \\
\hline $9: 00$ PM & 306 & 20 & 174 & 768 \\
\hline $10: 00$ PM & 264 & 9 & 156 & 361 \\
\hline $11: 00$ PM & 231 & 11 & 99 & 502 \\
\hline $12: 00$ AM & 232 & 0 & 124 & 252 \\
\hline $1: 00$ AM & 107 & 2 & 55 & 120 \\
\hline $2: 00$ AM & 94 & 3 & 43 & 31 \\
\hline $3: 00$ AM & 44 & 4 & 13 & 42 \\
\hline $4: 00$ AM & 27 & 0 & 5 & 0 \\
\hline $5: 00$ AM & 139 & 13 & 58 & 185 \\
\hline $6: 00$ AM & 246 & 3 & 101 & 736 \\
\hline $7: 00$ AM & 104 & 42 & 34 & 655 \\
\hline $8: 00$ AM & 15 & 9 & 9 & 416 \\
\hline $9: 00$ AM & 12 & 10 & 2 & 402 \\
\hline $10: 00$ AM & 4 & 0 & 3 & 629 \\
\hline $11: 00$ AM & 12 & 7 & 6 & 585 \\
\hline $12: 00$ PM & 2 & 0 & 4 & 653 \\
\hline \hline Total & 2469 & 187 & 1240 & 11603 \\
\hline & & & & \\
\hline
\end{tabular}


Table 6-15: Hourly Discrepancy Totals for Phase 7, Blanking Band = 2 sec., March 4 \& 5, 2001, Overcast

\begin{tabular}{|c|r|r|r|r|}
\cline { 2 - 5 } \multicolumn{1}{c|}{} & \multicolumn{2}{c|}{ System 1 } & \multicolumn{2}{c|}{ System 2 } \\
\hline \hline Hour Beginning & \multicolumn{1}{c|}{ L0V1 } & \multicolumn{1}{c|}{ L1V0 } & \multicolumn{1}{c|}{ L0V0 } \\
\hline 1:00 PM & 27 & 44 & 76 & 0 \\
\hline $2: 00 \mathrm{PM}$ & 60 & 68 & 190 & 16 \\
\hline $3: 00 \mathrm{PM}$ & 58 & 109 & 99 & 2 \\
\hline $4: 00 \mathrm{PM}$ & 56 & 42 & 5 & 26 \\
\hline $5: 00 \mathrm{PM}$ & 36 & 2 & 568 & 2 \\
\hline $6: 00 \mathrm{PM}$ & 139 & 2 & 221 & 0 \\
\hline $7: 00 \mathrm{PM}$ & 73 & 0 & 1514 & 3 \\
\hline 8:00 PM & 93 & 0 & 77 & 2 \\
\hline $9: 00 \mathrm{PM}$ & 88 & 4 & 65 & 0 \\
\hline $10: 00 \mathrm{PM}$ & 49 & 0 & 53 & 0 \\
\hline $11: 00 \mathrm{PM}$ & 46 & 0 & 11 & 0 \\
\hline $12: 00 \mathrm{AM}$ & 23 & 0 & 12 & 0 \\
\hline $1: 00 \mathrm{AM}$ & 74 & 0 & 2 & 0 \\
\hline $2: 00 \mathrm{AM}$ & 6 & 0 & 16 & 0 \\
\hline $3: 00 \mathrm{AM}$ & 3 & 3 & 2 & 3 \\
\hline $4: 00 \mathrm{AM}$ & 0 & 0 & & 0 \\
\hline $5: 00 \mathrm{AM}$ & 19 & 0 & 5 & 3 \\
\hline $6: 00 \mathrm{AM}$ & 28 & 0 & 11 & 0 \\
\hline $7: 00 \mathrm{AM}$ & 66 & 5 & 1703 & 0 \\
\hline 8:00 AM & 555 & 18 & 2830 & 42 \\
\hline $9: 00 \mathrm{AM}$ & 112 & 58 & 3004 & 16 \\
\hline $10: 00 \mathrm{AM}$ & 94 & 0 & 509 & 0 \\
\hline $11: 00 \mathrm{AM}$ & 139 & 49 & 193 & 29 \\
\hline $12: 00 \mathrm{PM}$ & 162 & 54 & 104 & 19 \\
\hline \hline Total & 2005 & 458 & 11271 & 164 \\
\hline & & & & \\
\hline
\end{tabular}


Table 6-16: Hourly Discrepancy Totals for Phase 8, Blanking Band = 2 sec., March 4 \& 5, 2001, Overcast

\begin{tabular}{|c|r|r|r|r|}
\cline { 2 - 5 } \multicolumn{1}{c|}{} & \multicolumn{2}{c|}{ System 1 } & \multicolumn{2}{c|}{ System 2 } \\
\hline \hline Hour Beginning & \multicolumn{1}{c|}{ L0V1 } & L1V0 & L0V1 & L1V0 \\
\hline \hline 1:00 PM & 10 & 219 & 0 & 266 \\
\hline $2: 00 \mathrm{PM}$ & 17 & 50 & 5 & 5 \\
\hline $3: 00 \mathrm{PM}$ & 21 & 249 & 3 & 325 \\
\hline 4:00 PM & 24 & 140 & 0 & 163 \\
\hline $5: 00 \mathrm{PM}$ & 17 & 145 & 0 & 167 \\
\hline $6: 00 \mathrm{PM}$ & 119 & 57 & 60 & 112 \\
\hline $7: 00 \mathrm{PM}$ & 397 & 102 & 413 & 88 \\
\hline 8:00 PM & 344 & 210 & 295 & 169 \\
\hline 9:00 PM & 356 & 303 & 299 & 278 \\
\hline $10: 00 \mathrm{PM}$ & 362 & 224 & 279 & 213 \\
\hline $11: 00 \mathrm{PM}$ & 338 & 512 & 244 & 502 \\
\hline $12: 00 \mathrm{AM}$ & 362 & 23 & 277 & 20 \\
\hline $1: 00 \mathrm{AM}$ & 283 & 331 & 179 & 340 \\
\hline $2: 00 \mathrm{AM}$ & 147 & 703 & 101 & 704 \\
\hline $3: 00 \mathrm{AM}$ & 65 & 16 & 47 & 16 \\
\hline $4: 00 \mathrm{AM}$ & 41 & 61 & 30 & 60 \\
\hline $5: 00 \mathrm{AM}$ & 94 & 763 & 78 & 768 \\
\hline $6: 00 \mathrm{AM}$ & 117 & 1312 & 97 & 1328 \\
\hline $7: 00 \mathrm{AM}$ & 143 & 231 & 67 & 172 \\
\hline 8:00 AM & 13 & 148 & 0 & 216 \\
\hline 9:00 AM & 27 & 88 & 0 & 173 \\
\hline $10: 00 \mathrm{AM}$ & 24 & 53 & 0 & 168 \\
\hline $11: 00 \mathrm{AM}$ & 46 & 200 & 3 & 289 \\
\hline $12: 00 \mathrm{PM}$ & 57 & 177 & 2 & 371 \\
\hline \hline Total & 3425 & 6317 & 2482 & 6914 \\
\hline & & & & \\
\hline
\end{tabular}


Large L1V0 discrepancies, such as these, are the cause of much concern. It appears that since the time that the System 2 video detector had been calibrated last, its detection on this approach had changed, making this a dangerous detector configuration. The next section of this chapter describes a data collection that was performed after the recalibration of each video detection system.

\section{Partly Sunny Conditions (After Recalibration)}

It was obvious after several data collections that each video detection system had certain characteristics that should be corrected. The results of the previous two data collections were presented to each of the vendors of the video detection systems, and they were given the chance to recalibrate their systems and try to alleviate as much of the discrepancies as possible. After this recalibration, another data collection was performed from 10:00 AM on April 2 10:00 AM on April 3, 2001. This data collection was made in order to be able to compare the differences between a data collection performed before the recalibration and a data collection after the recalibration.

Figure 6-17 through Figure 6-24 show the LOV1 and L1V0 discrepancies of all eight phases. Table 6-17 through Table 6-24 show the hourly discrepancy totals, in seconds, for each of the phases, as well. It is obvious that there are still some problem areas, but by looking at Table 6-25 and Table 6-26, it is also obvious that there has been quite an improvement over the pre-calibration data. The problem area for System 2 on phase 6 has been alleviated. In other words, there was a problem with this video detection zone that was fixed during the recalibration. The effects of calibration will be discussed in the next section. 


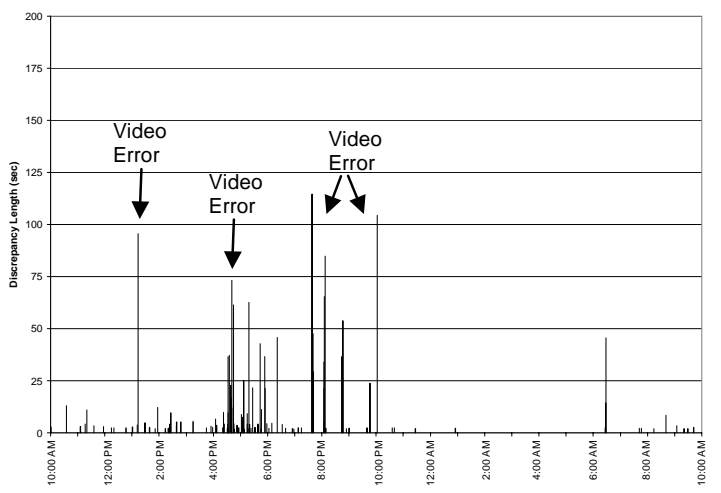

a) System 1 LOV1

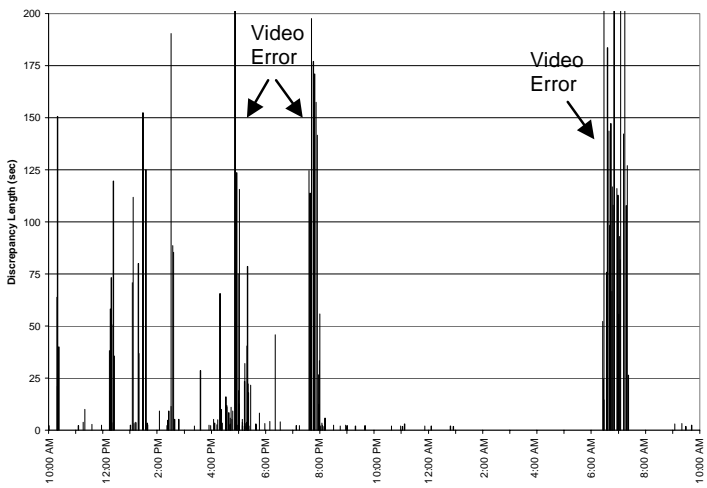

c) System 2 LOV1

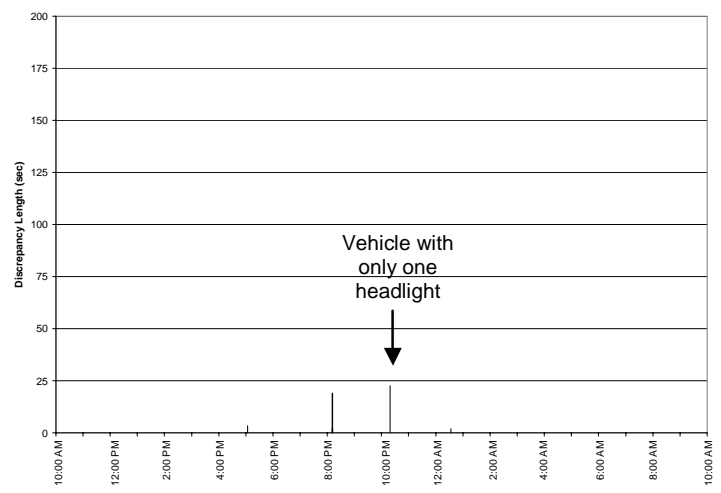

b) System 1 L1V0

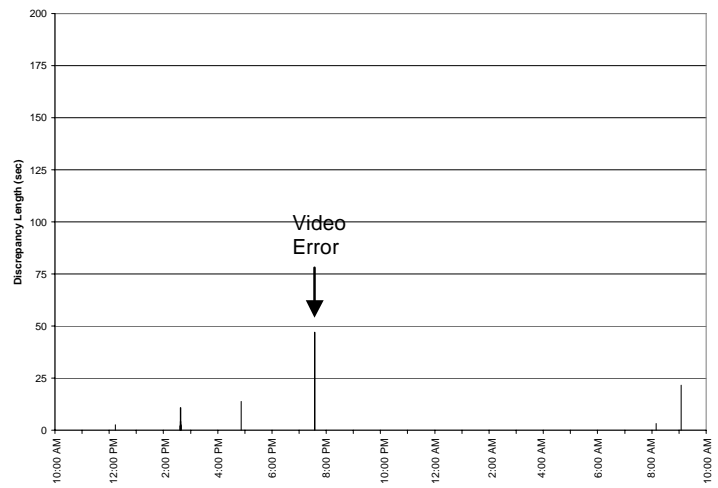

d) System 2 L1V0

Figure 6-17: Phase 1, Blanking Band = 2 sec., April 2 \& 3, 2001, After Recalibration, Partly Sunny 


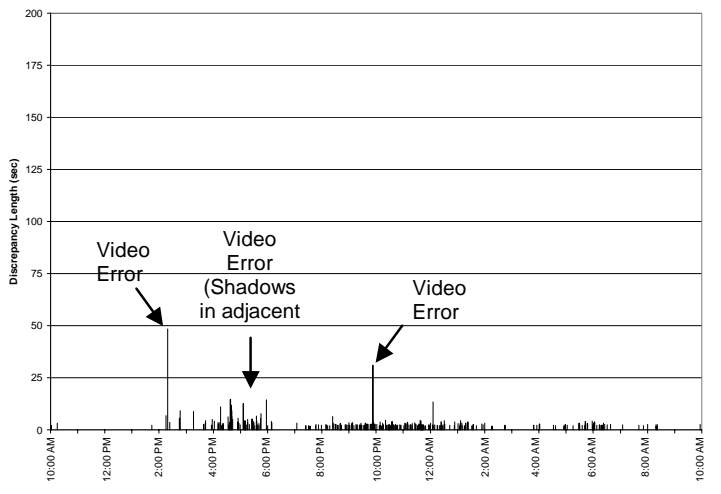

a) System 1 LOV1

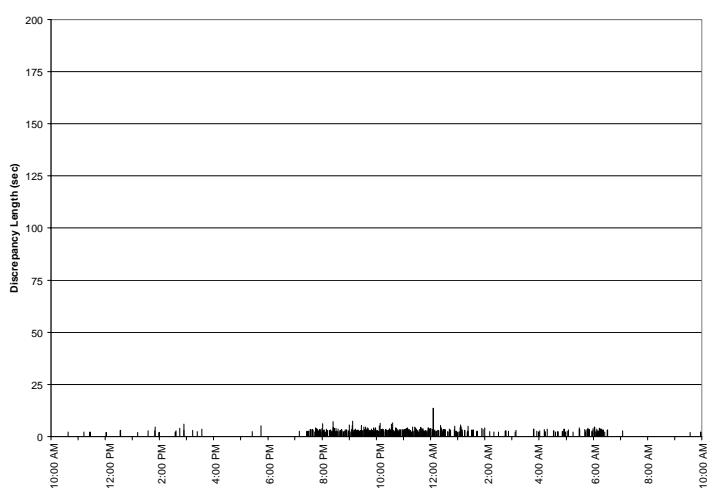

c) System 2 LOV1

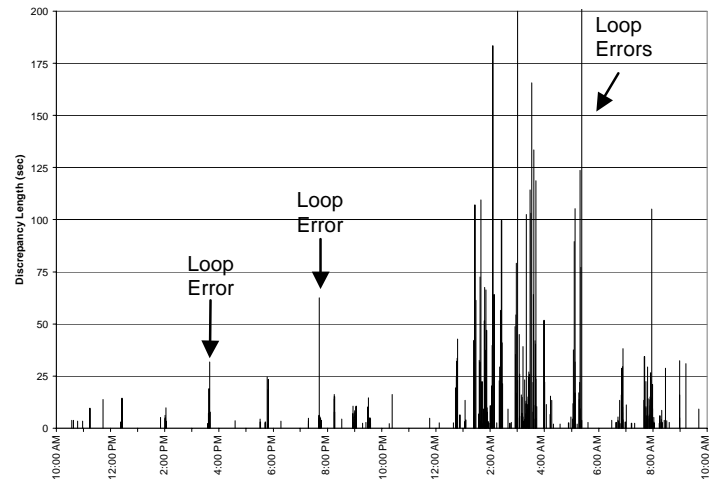

b) System 1 L1V0

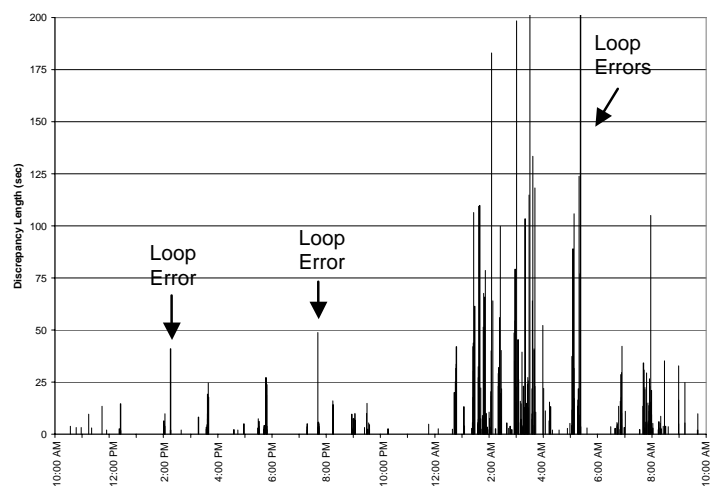

d) System 2 L1V0

Figure 6-18: Phase 2, Blanking Band = 2 sec., April 2 \& 3, 2001, After Recalibration, Partly Sunny 


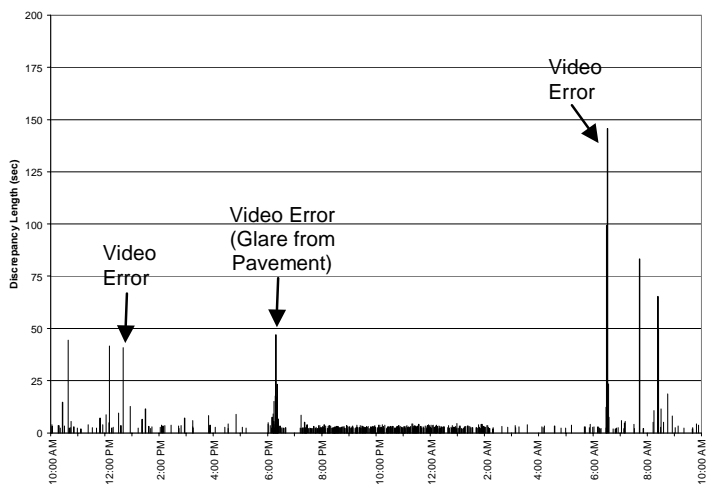

a) System 1 LOV1

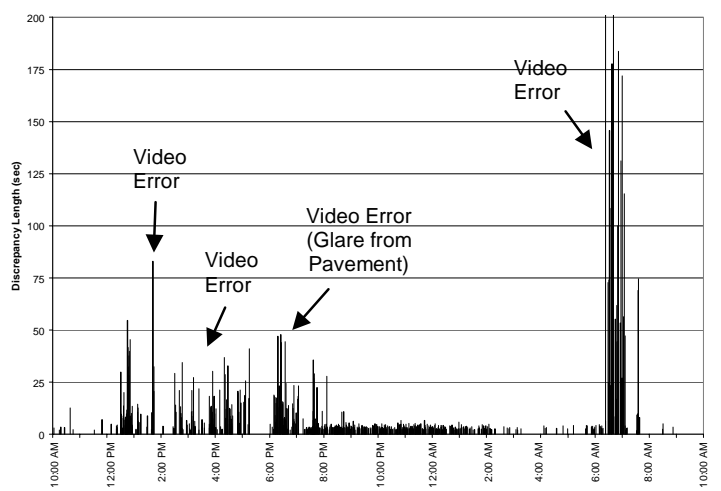

c) System 2 LOV1

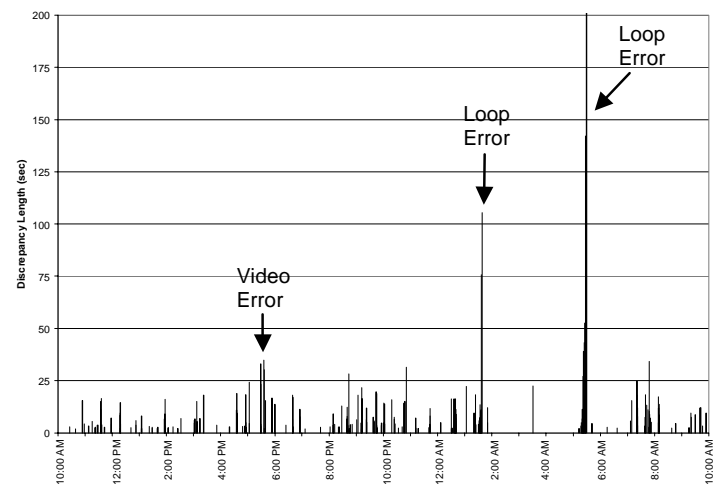

b) System 1 L1V0

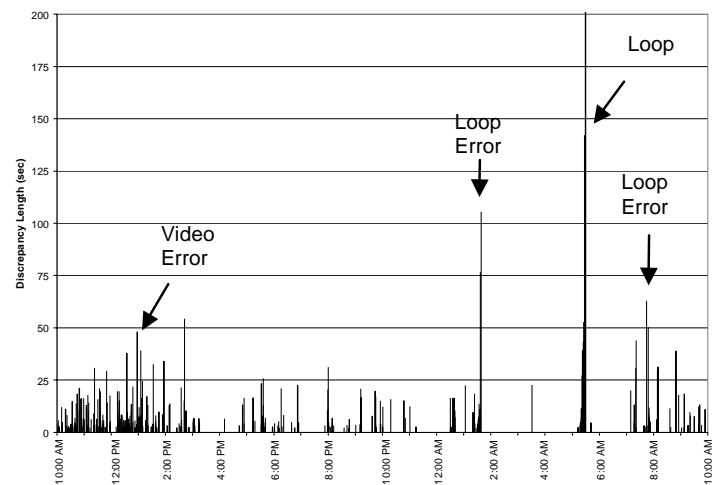

d) System 2 L1V0

Figure 6-19: Phase 3, Blanking Band = 2 sec., April 2 \& 3, 2001, After Recalibration, Partly Sunny 


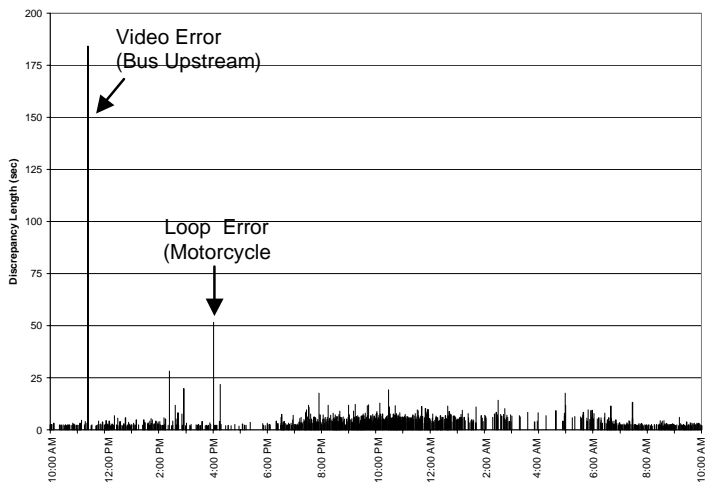

a) System 1 LOV1

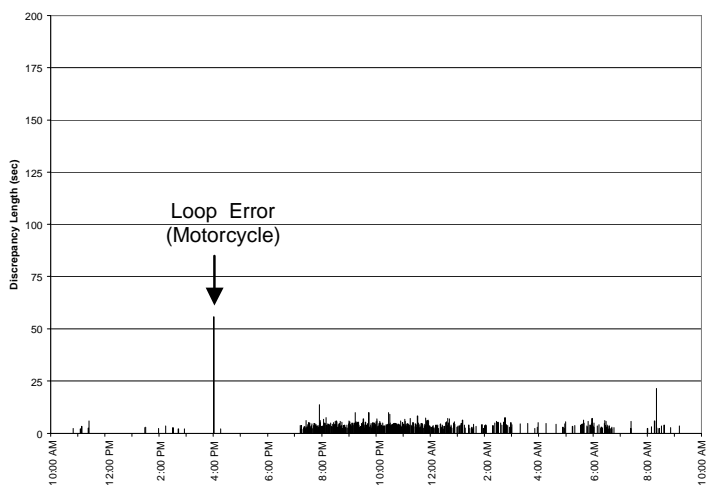

c) System 2 LOV1

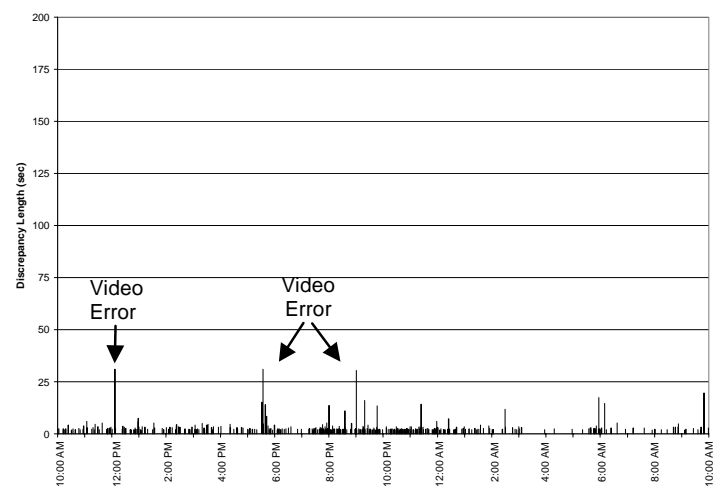

b) System 1 L1V0

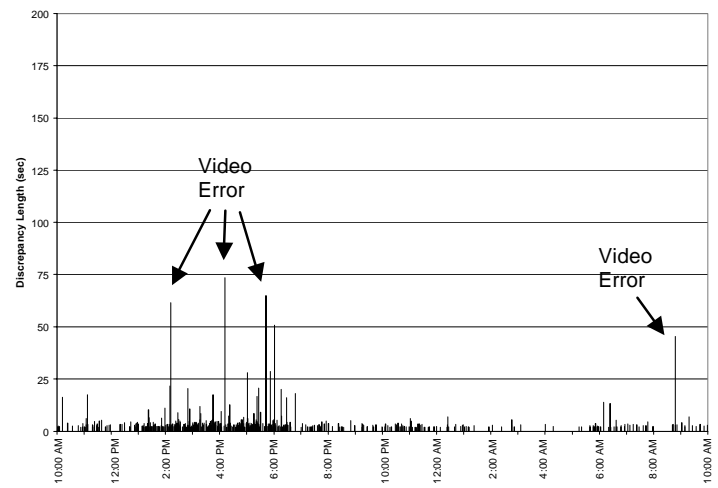

d) System 2 L1V0

Figure 6-20: Phase 4, Blanking Band = 2 sec., April 2 \& 3, 2001, After Recalibration, Partly Sunny 


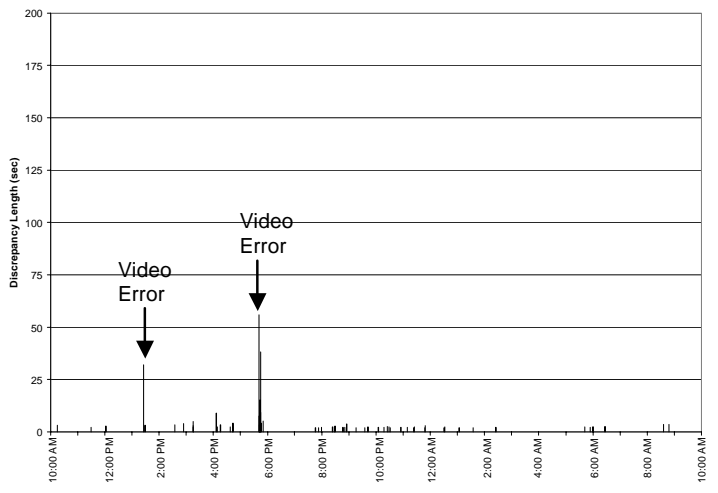

a) System 1 LOV1

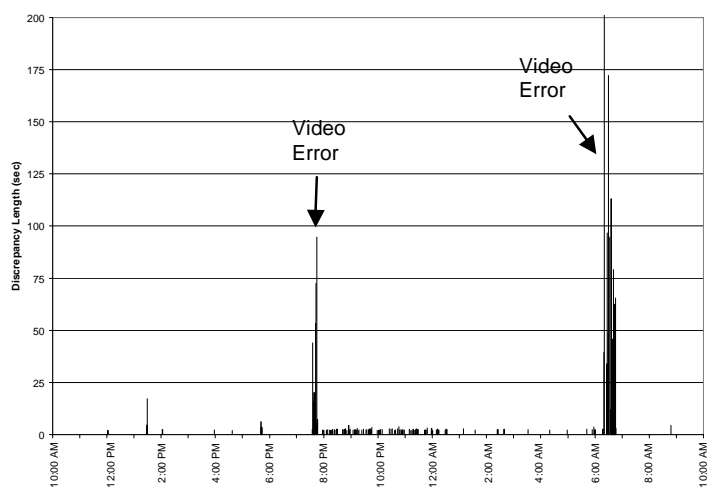

c) System 2 LOV1

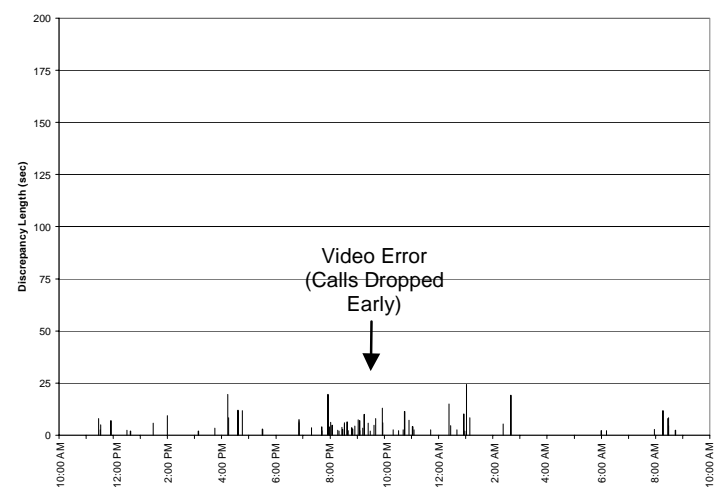

b) System 1 L1V0

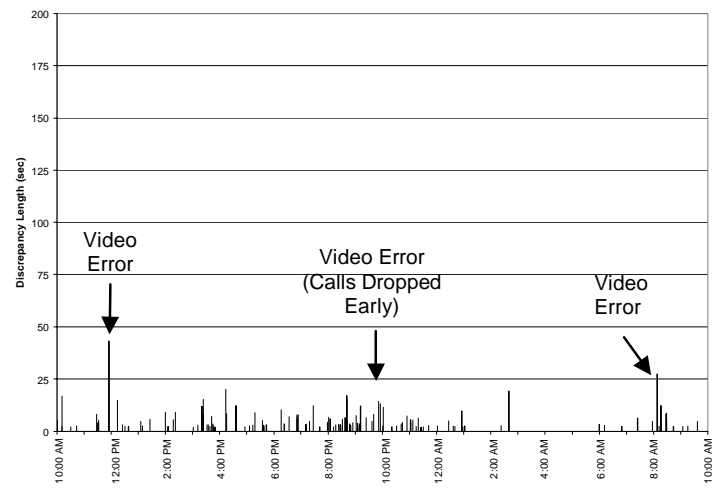

d) System 2 L1V0

Figure 6-21: Phase 5, Blanking Band = 2 sec., April 2 \& 3, 2001, After Recalibration, Partly Sunny 


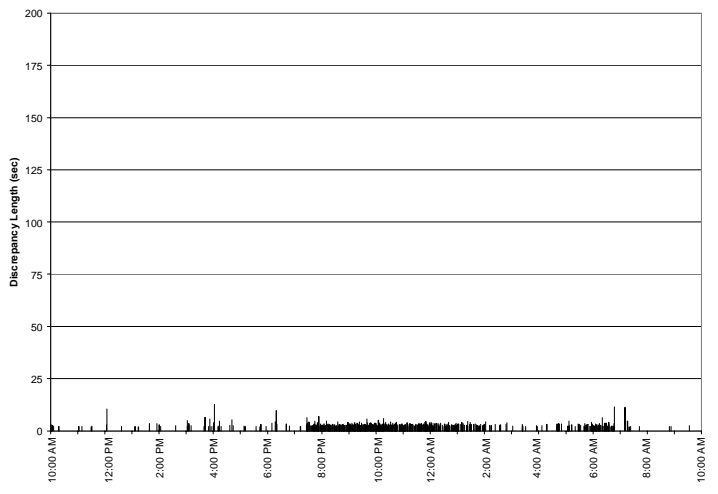

a) System 1 LOV1

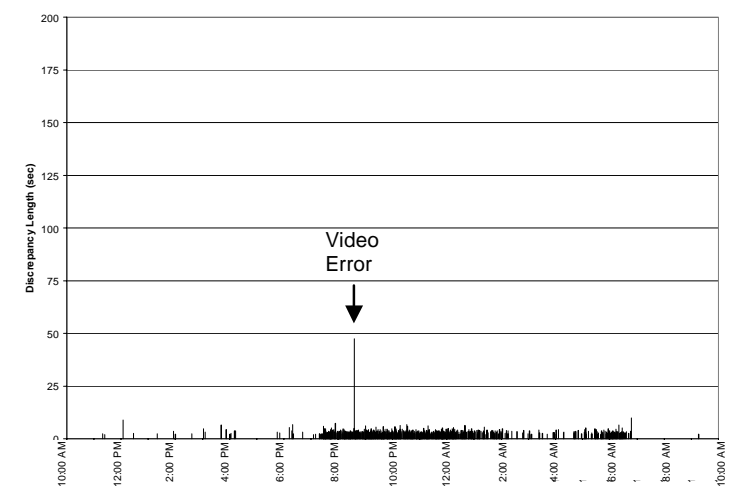

c) System 2 LOV1

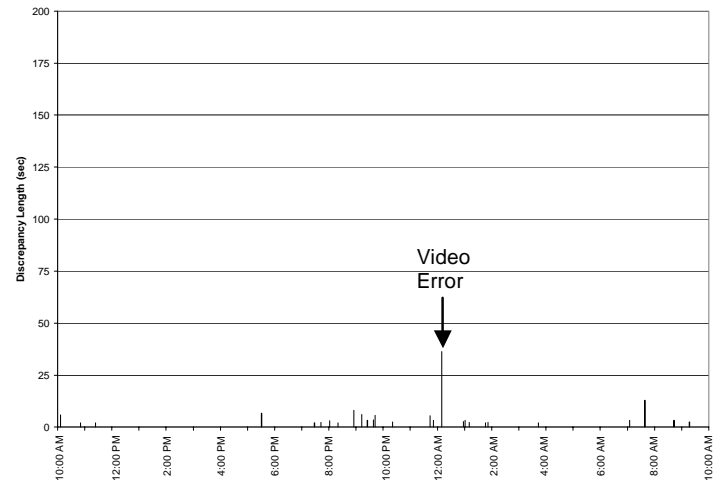

b) System 1 L1V0

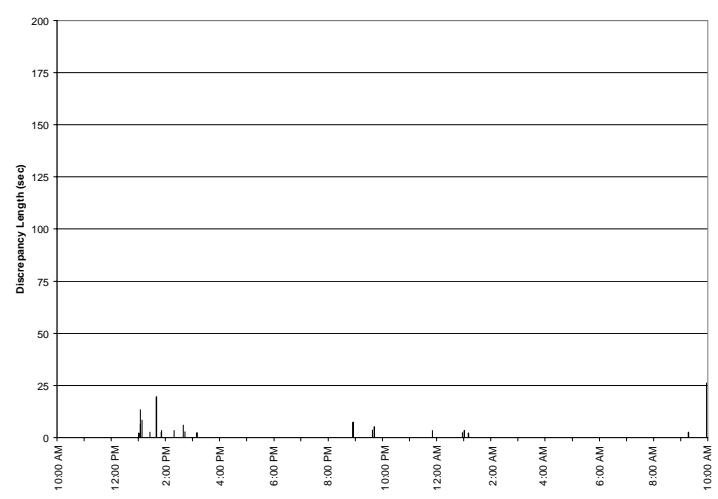

d) System 2 L1V0

Figure 6-22: Phase 6, Blanking Band = 2 sec., April $2 \&$ 3, 2001, After Recalibration, Partly Sunny 


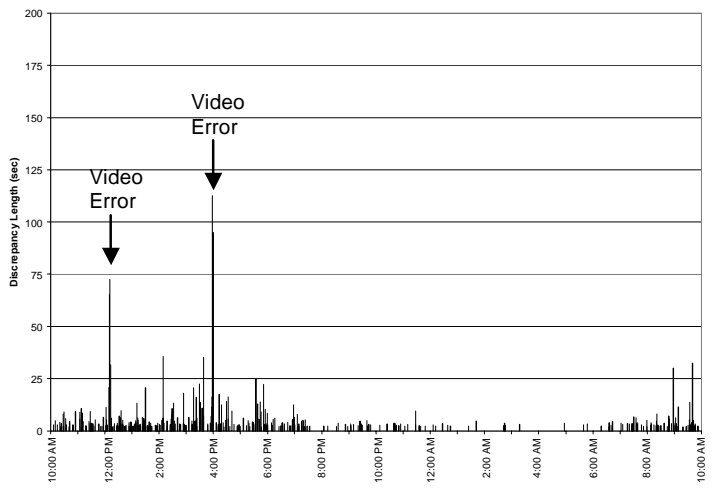

a) System 1 LOV1

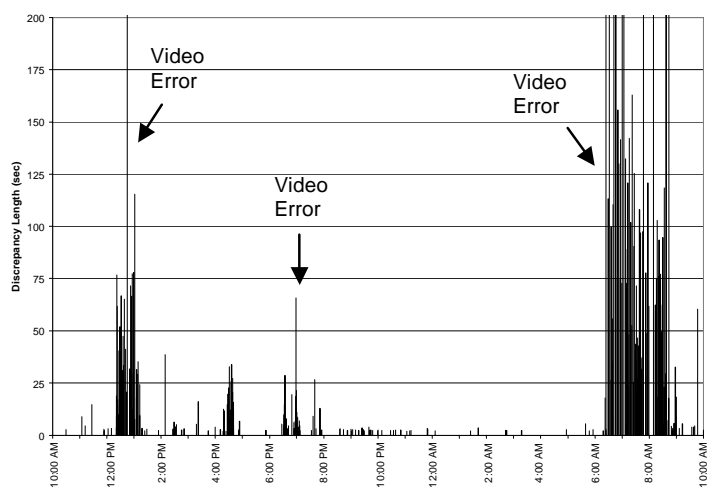

c) System 2 LOV1

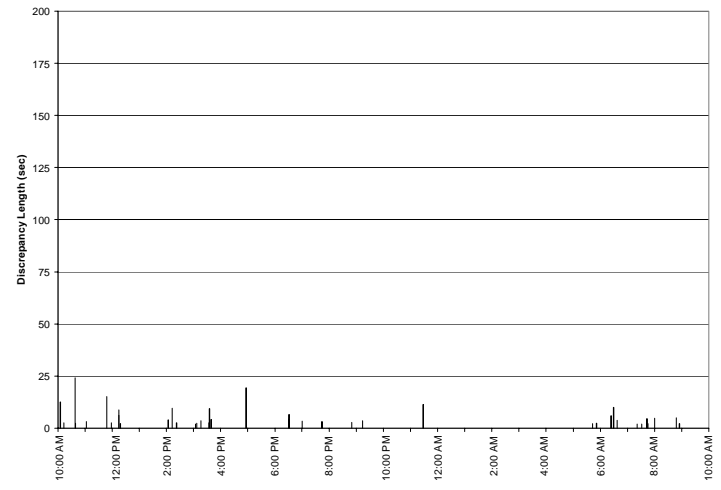

b) System 1 L1V0

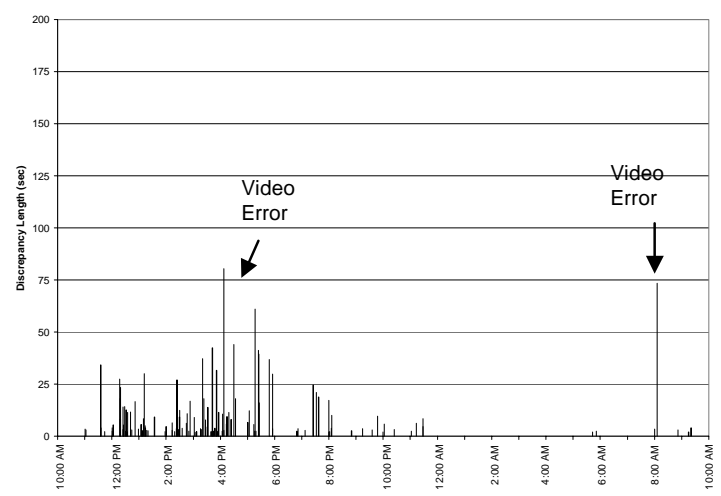

d) System 2 L1V0

Figure 6-23: Phase 7, Blanking Band = 2 sec., April 2 \& 3, 2001, After Recalibration, Partly Sunny 


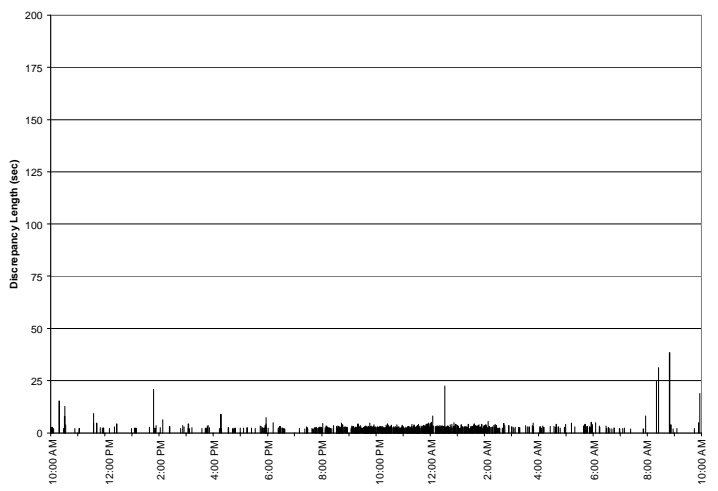

c) System 1 LOV1

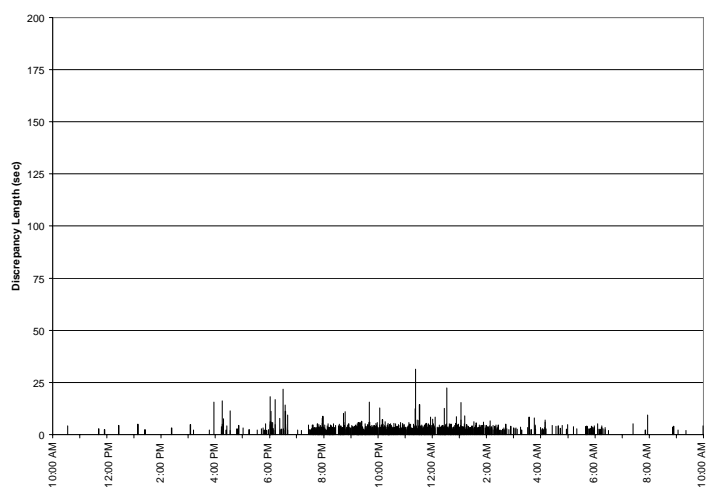

c) System 2 LOV1

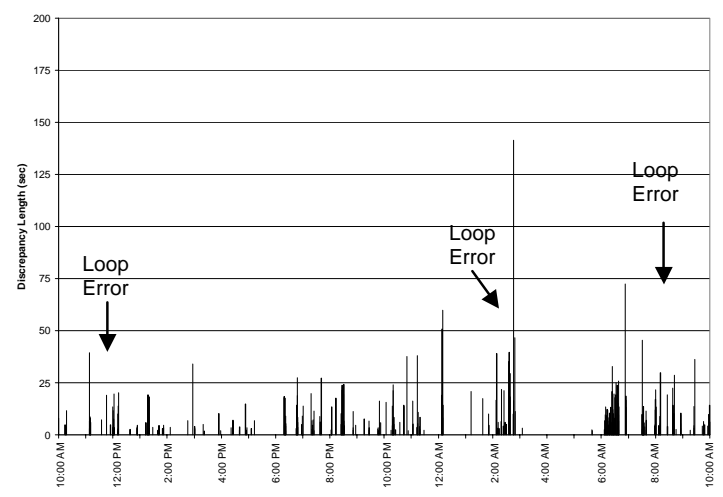

b) System 1 L1V0

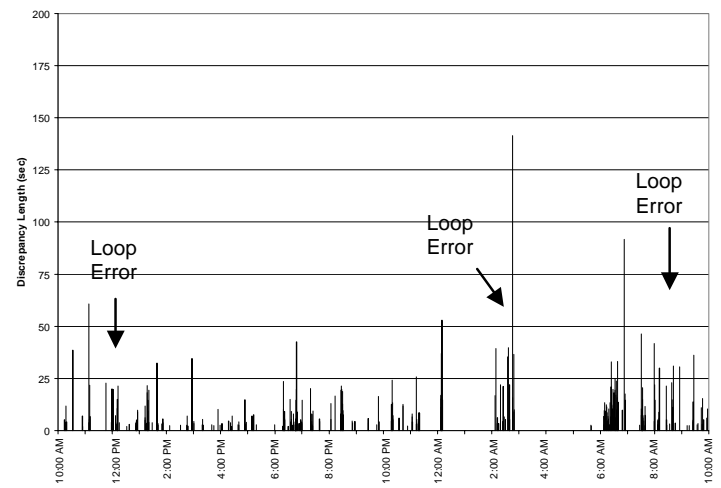

d) System 2 L1V0

Figure 6-24: Phase 8, Blanking Band = 2 sec., April 2 \& 3, 2001, After Recalibration, Partly Sunny 
Table 6-17: Hourly Discrepancy Totals for Phase 1, Blanking Band = $2 \mathrm{sec}$, April 2 \& 3, 2001, After Recalibration, Partly Sunny

\begin{tabular}{|c|r|r|r|r|}
\cline { 2 - 5 } \multicolumn{1}{c|}{} & \multicolumn{2}{c|}{ System 1 } & \multicolumn{2}{c|}{ System 2 } \\
\hline \hline Hour Beginning & \multicolumn{1}{c|}{ L0V1 } & \multicolumn{1}{c|}{ L1V0 } & L0V1 & \multicolumn{1}{c|}{ L1V0 } \\
\hline \hline 10:00 AM & 16 & 0 & 278 & 0 \\
\hline $11: 00$ AM & 25 & 0 & 22 & 0 \\
\hline $12: 00$ PM & 10 & 0 & 403 & 3 \\
\hline $1: 00$ PM & 124 & 0 & 596 & 0 \\
\hline $2: 00$ PM & 34 & 0 & 414 & 25 \\
\hline $3: 00$ PM & 19 & 0 & 36 & 0 \\
\hline $4: 00$ PM & 319 & 0 & 618 & 14 \\
\hline $5: 00$ PM & 288 & 3 & 433 & 0 \\
\hline $6: 00$ PM & 63 & 0 & 54 & 0 \\
\hline $7: 00$ PM & 197 & 0 & 1279 & 47 \\
\hline 8:00 PM & 333 & 21 & 27 & 0 \\
\hline $9: 00$ PM & 28 & 0 & 9 & 0 \\
\hline $10: 00$ PM & 109 & 23 & 4 & 0 \\
\hline $11: 00$ PM & 2 & 0 & 7 & 0 \\
\hline $12: 00$ AM & 2 & 2 & 6 & 0 \\
\hline $1: 00$ AM & 0 & 0 & 0 & 0 \\
\hline $2: 00$ AM & 0 & 0 & 0 & 0 \\
\hline $3: 00$ AM & 0 & 0 & 0 & 0 \\
\hline $4: 00$ AM & 0 & 0 & 0 & 0 \\
\hline $5: 00$ AM & 0 & 0 & 0 & 0 \\
\hline $6: 00$ AM & 63 & 0 & 2023 & 0 \\
\hline $7: 00$ AM & 5 & 0 & 1209 & 0 \\
\hline 8:00 AM & 11 & 0 & 0 & 3 \\
\hline $9: 00$ AM & 10 & 0 & 11 & 22 \\
\hline Total & 1647 & 49 & 7419 & 92 \\
\hline
\end{tabular}


Table 6-18: Hourly Discrepancy Totals for Phase 2, Blanking Band = $2 \mathrm{sec}$, April 2 \& 3, 2001, After Recalibration, Partly Sunny

\begin{tabular}{|c|r|r|r|r|}
\cline { 2 - 5 } \multicolumn{1}{c|}{} & \multicolumn{2}{c|}{ System 1 } & \multicolumn{2}{c|}{ System } \\
\hline \hline Hour Beginning & \multicolumn{1}{c|}{ L0V1 } & L1V0 & LOV1 & \multicolumn{1}{c|}{ L1V0 } \\
\hline \hline $10: 00$ AM & 5 & 14 & 2 & 11 \\
\hline $11: 00$ AM & 0 & 24 & 4 & 28 \\
\hline $12: 00$ PM & 0 & 17 & 5 & 19 \\
\hline $1: 00$ PM & 2 & 5 & 14 & 0 \\
\hline $2: 00$ PM & 74 & 36 & 18 & 82 \\
\hline $3: 00$ PM & 27 & 88 & 9 & 117 \\
\hline $4: 00$ PM & 112 & 4 & 0 & 10 \\
\hline $5: 00$ PM & 117 & 84 & 8 & 131 \\
\hline $6: 00$ PM & 10 & 3 & 0 & 0 \\
\hline $7: 00$ PM & 19 & 117 & 65 & 90 \\
\hline $8: 00$ PM & 58 & 101 & 134 & 80 \\
\hline $9: 00$ PM & 104 & 144 & 189 & 135 \\
\hline $10: 00$ PM & 80 & 18 & 178 & 3 \\
\hline $11: 00$ PM & 91 & 5 & 183 & 5 \\
\hline $12: 00$ AM & 67 & 202 & 116 & 194 \\
\hline $1: 00$ AM & 55 & 1025 & 71 & 1023 \\
\hline $2: 00$ AM & 4 & 1051 & 14 & 1047 \\
\hline $3: 00$ AM & 5 & 1597 & 13 & 1597 \\
\hline $4: 00$ AM & 14 & 81 & 32 & 81 \\
\hline $5: 00$ AM & 31 & 816 & 50 & 814 \\
\hline $6: 00$ AM & 37 & 225 & 57 & 227 \\
\hline $7: 00$ AM & 10 & 452 & 3 & 489 \\
\hline 8:00 AM & 9 & 140 & 0 & 146 \\
\hline $9: 00$ AM & 3 & 40 & 4 & 42 \\
\hline Total & 931 & 6248 & 1165 & 6329 \\
\hline
\end{tabular}


Table 6-19: Hourly Discrepancy Totals for Phase 3, Blanking Band $=2 \mathrm{sec}$., April 2 \& 3, 2001, After Recalibration, Partly Sunny

\begin{tabular}{|c|r|r|r|r|}
\cline { 2 - 5 } \multicolumn{1}{c|}{} & \multicolumn{2}{c|}{ System 1 } & \multicolumn{2}{c|}{ System 2 } \\
\hline \hline Hour Beginning & \multicolumn{1}{c|}{ L0V1 } & \multicolumn{1}{c|}{ L1V0 } & \multicolumn{1}{c|}{ L0V1 } & L1V0 \\
\hline \hline 10:00 AM & 94 & 25 & 27 & 263 \\
\hline $11: 00$ AM & 22 & 90 & 9 & 337 \\
\hline $12: 00$ PM & 127 & 60 & 649 & 363 \\
\hline $1: 00$ PM & 32 & 62 & 262 & 300 \\
\hline $2: 00$ PM & 35 & 17 & 181 & 178 \\
\hline $3: 00$ PM & 25 & 69 & 353 & 45 \\
\hline $4: 00$ PM & 21 & 101 & 388 & 47 \\
\hline $5: 00$ PM & 5 & 206 & 144 & 148 \\
\hline $6: 00$ PM & 224 & 71 & 696 & 86 \\
\hline $7: 00$ PM & 92 & 5 & 341 & 65 \\
\hline $8: 00$ PM & 125 & 114 & 266 & 37 \\
\hline $9: 00$ PM & 125 & 173 & 169 & 115 \\
\hline $10: 00$ PM & 120 & 142 & 171 & 52 \\
\hline $11: 00$ PM & 133 & 38 & 189 & 15 \\
\hline $12: 00$ AM & 104 & 134 & 131 & 119 \\
\hline $1: 00$ AM & 83 & 359 & 93 & 345 \\
\hline $2: 00$ AM & 28 & 0 & 37 & 0 \\
\hline $3: 00$ AM & 13 & 22 & 10 & 22 \\
\hline $4: 00$ AM & 17 & 0 & 20 & 0 \\
\hline $5: 00$ AM & 22 & 670 & 42 & 669 \\
\hline $6: 00$ AM & 314 & 5 & 1994 & 0 \\
\hline $7: 00$ AM & 110 & 227 & 600 & 275 \\
\hline 8:00 AM & 177 & 71 & 11 & 143 \\
\hline $9: 00$ AM & 21 & 65 & 0 & 94 \\
\hline Total & 2044 & 2660 & 6783 & 3625 \\
\hline & & & & \\
\hline
\end{tabular}


Table 6-20: Hourly Discrepancy Totals for Phase 4, Blanking Band $=2 \mathrm{sec}$., April 2 \& 3, 2001, After Recalibration, Partly Sunny

\begin{tabular}{|c|r|r|r|r|}
\cline { 2 - 5 } \multicolumn{1}{c|}{} & \multicolumn{2}{c|}{ System 1 } & \multicolumn{2}{c|}{ System 2 } \\
\hline Hour Beginning & \multicolumn{1}{c|}{ L0V1 } & L1V0 & L0V1 & L1V0 \\
\hline \hline 10:00 AM & 72 & 34 & 2 & 42 \\
\hline $11: 00$ AM & 248 & 45 & 14 & 76 \\
\hline $12: 00$ PM & 124 & 88 & 0 & 44 \\
\hline $1: 00$ PM & 105 & 28 & 8 & 116 \\
\hline $2: 00$ PM & 139 & 44 & 10 & 285 \\
\hline $3: 00$ PM & 46 & 46 & 0 & 189 \\
\hline $4: 00$ PM & 105 & 27 & 58 & 248 \\
\hline $5: 00$ PM & 24 & 108 & 0 & 349 \\
\hline $6: 00$ PM & 107 & 32 & 0 & 182 \\
\hline $7: 00$ PM & 291 & 79 & 181 & 56 \\
\hline $8: 00$ PM & 336 & 92 & 235 & 28 \\
\hline $9: 00$ PM & 381 & 133 & 275 & 32 \\
\hline $10: 00$ PM & 448 & 85 & 284 & 52 \\
\hline $11: 00$ PM & 399 & 97 & 257 & 52 \\
\hline $12: 00$ AM & 261 & 42 & 165 & 30 \\
\hline $1: 00$ AM & 136 & 32 & 81 & 13 \\
\hline $2: 00$ AM & 152 & 40 & 100 & 16 \\
\hline $3: 00$ AM & 45 & 8 & 24 & 3 \\
\hline $4: 00$ AM & 65 & 5 & 24 & 6 \\
\hline $5: 00$ AM & 123 & 52 & 81 & 31 \\
\hline $6: 00$ AM & 179 & 30 & 92 & 57 \\
\hline $7: 00$ AM & 116 & 8 & 8 & 27 \\
\hline 8:00 AM & 112 & 21 & 48 & 58 \\
\hline $9: 00$ AM & 110 & 39 & 4 & 32 \\
\hline Total & 4014 & 1175 & 1948 & 1992 \\
\hline & & & & \\
\hline
\end{tabular}


Table 6-21: Hourly Discrepancy Totals for Phase 5, Blanking Band $=2 \mathrm{sec}$., April 2 \& 3, 2001, After Recalibration, Partly Sunny

\begin{tabular}{|c|r|r|r|r|}
\cline { 2 - 5 } \multicolumn{1}{c|}{} & \multicolumn{2}{c|}{ System 1 } & \multicolumn{2}{c|}{ System 2 } \\
\hline \hline Hour Beginning & L0V1 & L1V0 & L0V1 & L1V0 \\
\hline \hline 10:00 AM & 3 & 0 & 0 & 29 \\
\hline $11: 00 \mathrm{AM}$ & 2 & 23 & 0 & 61 \\
\hline $12: 00 \mathrm{PM}$ & 3 & 4 & 2 & 23 \\
\hline $1: 00 \mathrm{PM}$ & 35 & 15 & 22 & 22 \\
\hline $2: 00 \mathrm{PM}$ & 7 & 0 & 2 & 17 \\
\hline $3: 00 \mathrm{PM}$ & 10 & 5 & 2 & 91 \\
\hline $4: 00 \mathrm{PM}$ & 23 & 64 & 2 & 59 \\
\hline $5: 00 \mathrm{PM}$ & 146 & 6 & 26 & 28 \\
\hline $6: 00 \mathrm{PM}$ & 0 & 20 & 0 & 50 \\
\hline $7: 00 \mathrm{PM}$ & 6 & 33 & 378 & 27 \\
\hline $8: 00 \mathrm{PM}$ & 16 & 52 & 43 & 89 \\
\hline $9: 00 \mathrm{PM}$ & 6 & 69 & 38 & 78 \\
\hline $10: 00 \mathrm{PM}$ & 18 & 26 & 43 & 34 \\
\hline $11: 00 \mathrm{PM}$ & 11 & 13 & 38 & 31 \\
\hline $12: 00 \mathrm{AM}$ & 4 & 34 & 18 & 24 \\
\hline $1: 00 \mathrm{AM}$ & 4 & 33 & 5 & 3 \\
\hline $2: 00 \mathrm{AM}$ & 2 & 24 & 7 & 22 \\
\hline $3: 00 \mathrm{AM}$ & 0 & 0 & 2 & 0 \\
\hline $4: 00 \mathrm{AM}$ & 0 & 0 & 4 & 0 \\
\hline $5: 00 \mathrm{AM}$ & 7 & 2 & 11 & 3 \\
\hline $6: 00 \mathrm{AM}$ & 5 & 2 & 1438 & 5 \\
\hline $7: 00 \mathrm{AM}$ & 0 & 3 & 0 & 11 \\
\hline 8:00 AM & 7 & 35 & 4 & 69 \\
\hline $9: 00 \mathrm{AM}$ & 0 & 0 & 0 & 10 \\
\hline Total & 317 & 465 & 2086 & 775 \\
\hline & & & & \\
\hline
\end{tabular}


Table 6-22: Hourly Discrepancy Totals for Phase 6, Blanking Band $=2 \mathrm{sec}$., April 2 \& 3, 2001, After Recalibration, Partly Sunny

\begin{tabular}{|c|r|r|r|r|}
\cline { 2 - 5 } \multicolumn{1}{c|}{} & \multicolumn{2}{c|}{ System 1 } & \multicolumn{2}{c|}{ System 2 } \\
\hline \hline Hour Beginning & L0V1 & L1V0 & L0V1 & \multicolumn{1}{c|}{ L1V0 } \\
\hline \hline $10: 00 \mathrm{AM}$ & 12 & 8 & 0 & 0 \\
\hline $11: 00 \mathrm{AM}$ & 9 & 2 & 4 & 0 \\
\hline $12: 00 \mathrm{PM}$ & 16 & 0 & 12 & 0 \\
\hline $1: 00 \mathrm{PM}$ & 13 & 0 & 6 & 58 \\
\hline $2: 00 \mathrm{PM}$ & 8 & 0 & 5 & 12 \\
\hline $3: 00 \mathrm{PM}$ & 37 & 0 & 19 & 4 \\
\hline $4: 00 \mathrm{PM}$ & 38 & 0 & 12 & 0 \\
\hline $5: 00 \mathrm{PM}$ & 14 & 7 & 6 & 0 \\
\hline $6: 00 \mathrm{PM}$ & 31 & 0 & 24 & 0 \\
\hline $7: 00 \mathrm{PM}$ & 118 & 5 & 184 & 0 \\
\hline $8: 00 \mathrm{PM}$ & 180 & 13 & 309 & 7 \\
\hline $9: 00 \mathrm{PM}$ & 223 & 19 & 312 & 9 \\
\hline $10: 00 \mathrm{PM}$ & 213 & 3 & 300 & 0 \\
\hline $11: 00 \mathrm{PM}$ & 207 & 9 & 278 & 3 \\
\hline $12: 00 \mathrm{AM}$ & 155 & 39 & 239 & 2 \\
\hline $1: 00 \mathrm{AM}$ & 96 & 10 & 140 & 6 \\
\hline $2: 00 \mathrm{AM}$ & 33 & 0 & 45 & 0 \\
\hline $3: 00 \mathrm{AM}$ & 17 & 2 & 28 & 0 \\
\hline $4: 00 \mathrm{AM}$ & 27 & 0 & 47 & 0 \\
\hline $5: 00 \mathrm{AM}$ & 80 & 0 & 126 & 0 \\
\hline $6: 00 \mathrm{AM}$ & 121 & 0 & 132 & 0 \\
\hline $7: 00 \mathrm{AM}$ & 22 & 16 & 0 & 0 \\
\hline 8:00 AM & 4 & 3 & 0 & 0 \\
\hline $9: 00 \mathrm{AM}$ & 3 & 2 & 2 & 29 \\
\hline Total & 1673 & 135 & 2227 & 102 \\
\hline & & & & \\
\hline
\end{tabular}


Table 6-23: Hourly Discrepancy Totals for Phase 7, Blanking Band = 2 sec., April 2 \& 3, 2001, After Recalibration, Partly Sunny

\begin{tabular}{|c|r|r|r|r|}
\cline { 2 - 5 } \multicolumn{1}{c|}{} & \multicolumn{2}{c|}{ System 1 } & \multicolumn{2}{c|}{ System 2 } \\
\hline \hline Hour Beginning & L0V1 & L1V0 & L0V1 & \multicolumn{1}{c|}{ L1V0 } \\
\hline \hline 10:00 AM & 68 & 42 & 3 & 0 \\
\hline $11: 00 \mathrm{AM}$ & 103 & 21 & 35 & 48 \\
\hline $12: 00 \mathrm{PM}$ & 321 & 17 & 1560 & 198 \\
\hline $1: 00 \mathrm{PM}$ & 151 & 0 & 326 & 80 \\
\hline $2: 00 \mathrm{PM}$ & 167 & 16 & 76 & 111 \\
\hline $3: 00 \mathrm{PM}$ & 424 & 24 & 28 & 202 \\
\hline $4: 00 \mathrm{PM}$ & 132 & 19 & 365 & 193 \\
\hline $5: 00 \mathrm{PM}$ & 169 & 0 & 5 & 258 \\
\hline $6: 00 \mathrm{PM}$ & 77 & 11 & 278 & 6 \\
\hline $7: 00 \mathrm{PM}$ & 51 & 7 & 140 & 87 \\
\hline 8:00 PM & 16 & 3 & 14 & 19 \\
\hline $9: 00 \mathrm{PM}$ & 36 & 4 & 31 & 19 \\
\hline $10: 00 \mathrm{PM}$ & 28 & 0 & 14 & 9 \\
\hline $11: 00 \mathrm{PM}$ & 25 & 11 & 16 & 22 \\
\hline $12: 00 \mathrm{AM}$ & 14 & 0 & 2 & 0 \\
\hline $1: 00 \mathrm{AM}$ & 7 & 0 & 6 & 0 \\
\hline $2: 00 \mathrm{AM}$ & 9 & 0 & 5 & 0 \\
\hline $3: 00 \mathrm{AM}$ & 3 & 0 & 2 & 0 \\
\hline $4: 00 \mathrm{AM}$ & 4 & 0 & 3 & 0 \\
\hline $5: 00 \mathrm{AM}$ & 6 & 5 & 10 & 5 \\
\hline $6: 00 \mathrm{AM}$ & 15 & 20 & 2144 & 0 \\
\hline 7:00 AM & 62 & 16 & 3044 & 4 \\
\hline 8:00 AM & 112 & 7 & 1991 & 76 \\
\hline $9: 00 \mathrm{AM}$ & 107 & 0 & 102 & 6 \\
\hline Total & 1998 & 221 & 10098 & 1337 \\
\hline & & & & \\
\hline
\end{tabular}


Table 6-24: Hourly Discrepancy Totals for Phase 8, Blanking Band = 2 sec., April 2 \& 3, 2001, After Recalibration, Partly Sunny

\begin{tabular}{|c|c|c|c|c|}
\hline & \multicolumn{2}{|c|}{ System 1} & \multicolumn{2}{|c|}{ System 2} \\
\hline Hour Beginning & LOV1 & L1V0 & LOV1 & L1V0 \\
\hline$\overline{10: 00 \mathrm{AM}}$ & 51 & 32 & 4 & 76 \\
\hline 11:00 AM & 30 & 140 & 5 & 154 \\
\hline 12:00 PM & 12 & 128 & 4 & 187 \\
\hline 1:00 PM & 36 & 135 & 7 & 185 \\
\hline $2: 00 \mathrm{PM}$ & 20 & 45 & 3 & 56 \\
\hline 3:00 PM & 34 & 27 & 25 & 36 \\
\hline 4:00 PM & 22 & 40 & 61 & 55 \\
\hline 5:00 PM & 47 & 10 & 28 & 26 \\
\hline 6:00 PM & 26 & 202 & 186 & 280 \\
\hline 7:00 PM & 59 & 120 & 193 & 82 \\
\hline 8:00 PM & 125 & 243 & 297 & 188 \\
\hline 9:00 PM & 177 & 57 & 379 & 33 \\
\hline 10:00 PM & 182 & 188 & 370 & 116 \\
\hline 11:00 PM & 224 & 113 & 444 & 85 \\
\hline $12: 00 \mathrm{AM}$ & 262 & 185 & 409 & 174 \\
\hline 1:00 AM & 150 & 53 & 278 & 0 \\
\hline $2: 00 \mathrm{AM}$ & 87 & 482 & 112 & 478 \\
\hline 3:00 AM & 36 & 3 & 54 & 0 \\
\hline 4:00 AM & 42 & 0 & 58 & 0 \\
\hline 5:00 AM & 49 & 5 & 60 & 5 \\
\hline $6: 00 \mathrm{AM}$ & 32 & 663 & 38 & 672 \\
\hline 7:00 AM & 17 & 192 & 17 & 218 \\
\hline $8: 00 \mathrm{AM}$ & 100 & 238 & 7 & 259 \\
\hline 9:00 AM & 28 & 119 & 8 & 121 \\
\hline Total & 1819 & 3299 & 3040 & 3364 \\
\hline
\end{tabular}

Table 6-25: 24 Hour Discrepancy Totals (in seconds) Before Recalibration, Blanking Band $=2 \mathrm{sec}$.

\begin{tabular}{|c|c|c|c|c|c|}
\hline \multirow{3}{*}{\multicolumn{2}{|c|}{$\begin{array}{l}\text { Data Collection Date } \\
\text { System } \\
\text { Discrepancy Type }\end{array}$}} & \multicolumn{4}{|c|}{ March 4 \& 5, 2001} \\
\hline & & \multicolumn{2}{|c|}{ System 1} & \multicolumn{2}{|c|}{ System 2} \\
\hline & & \begin{tabular}{l|l} 
L0V1 & \\
\end{tabular} & L1V0 & \begin{tabular}{l|l} 
LoV1 \\
\end{tabular} & L1V0 \\
\hline \multirow{8}{*}{ 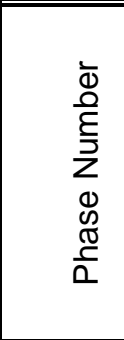 } & 1 & 1138 & 187 & 7284 & 14 \\
\hline & 2 & 1541 & 3307 & 2313 & 3264 \\
\hline & 3 & 3216 & 3328 & 3096 & 4225 \\
\hline & 4 & 4815 & 929 & 2290 & 1190 \\
\hline & 5 & 1037 & 797 & 1159 & 1083 \\
\hline & 6 & 2469 & 187 & 1240 & 11603 \\
\hline & 7 & 2005 & 458 & 11271 & 164 \\
\hline & 8 & 3425 & 6317 & 2482 & 6914 \\
\hline \multicolumn{2}{|c|}{ Total } & 19646 & 15510 & 31135 & 28457 \\
\hline
\end{tabular}


Table 6-26: 24 Hour Discrepancy Totals (in seconds) After Recalibration, Blanking Band $=2 \mathrm{sec}$.

\begin{tabular}{|c|c|c|c|c|c|}
\hline \multirow{3}{*}{\multicolumn{2}{|c|}{$\begin{array}{l}\text { Data Collection Date } \\
\text { System } \\
\text { Discrepancy Type }\end{array}$}} & \multicolumn{4}{|c|}{ April 2 \& 3, 2001} \\
\hline & & \multicolumn{2}{|c|}{ System 1} & \multicolumn{2}{|c|}{ System 2} \\
\hline & & LOV1 & L1V0 & \begin{tabular}{l|} 
LOV1 \\
\end{tabular} & L1V0 \\
\hline \multirow{8}{*}{ 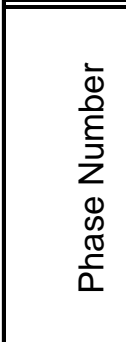 } & 1 & 1647 & $\overline{49}$ & 7419 & 92 \\
\hline & 2 & 931 & 6248 & 1165 & 6329 \\
\hline & 3 & 2044 & 2660 & 6783 & 3625 \\
\hline & 4 & 4014 & 1175 & 1948 & 1992 \\
\hline & 5 & 317 & 465 & 2086 & 775 \\
\hline & 6 & 1673 & 135 & 2227 & 102 \\
\hline & $\overline{7}$ & 1998 & 221 & 10098 & 1337 \\
\hline & 8 & 1819 & 3299 & 3040 & 3364 \\
\hline \multicolumn{2}{|c|}{$\overline{\text { Total }}$} & 14443 & 14252 & 34766 & 17616 \\
\hline
\end{tabular}

Mostly Sunny Conditions (After Installation of New System 2 Cameras)

Because all four cameras at the intersection are designed for use with System 1, it was decided that two more cameras, designed for use with System 2, should be installed at the intersection in order to determine whether the performance of System 2 would improve. The new cameras were installed facing the eastbound and the southbound approaches (served by phases 1, 3, 6, and 8 ). The video from the new System 2 cameras was fed through only System 2 on the eastbound and southbound approaches. System 1 still ran with the video feed from its own cameras on these approaches. Figure 6-25 through Figure 6-28 show the L0V1 and L1V0 discrepancies of all four of the phases affected by the new camera installation. Table 6-27 through Table 6-30 show the hourly discrepancy totals, in seconds, for each of these phases, as well. 


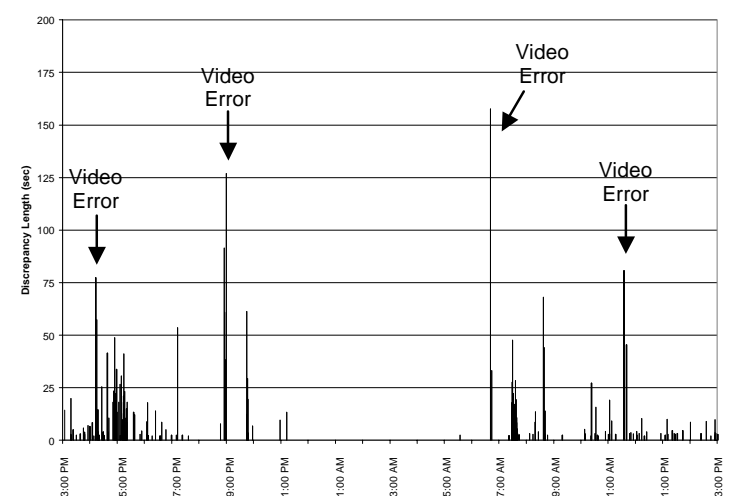

a) System 1 LOV1

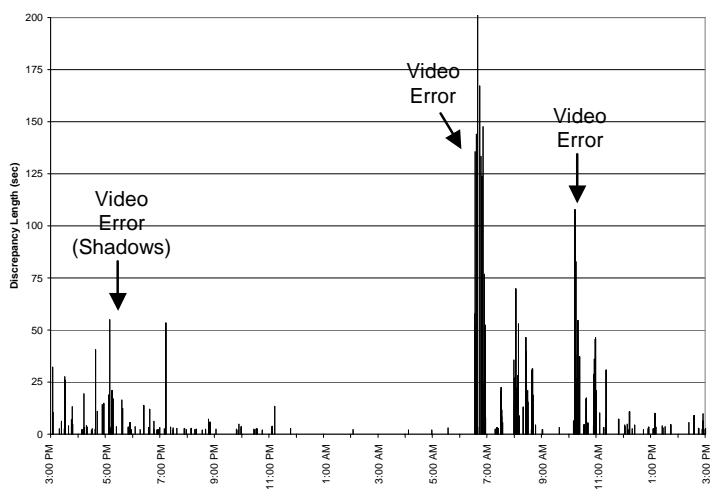

c) System 2 LOV1

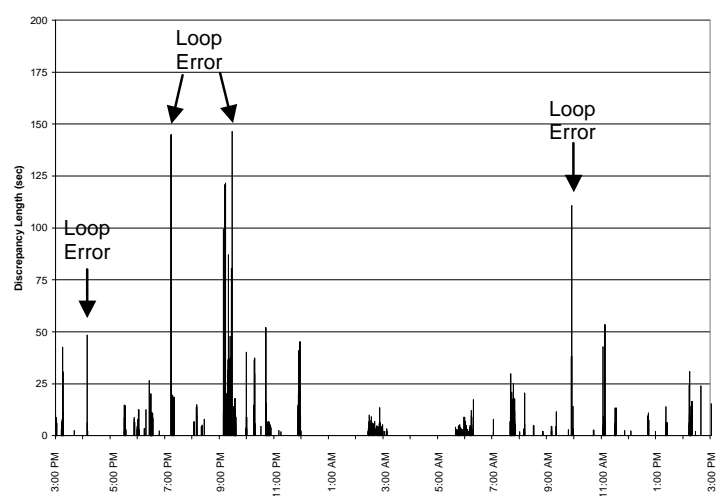

b) System $1 \mathrm{~L} 1 \mathrm{~V} 0$

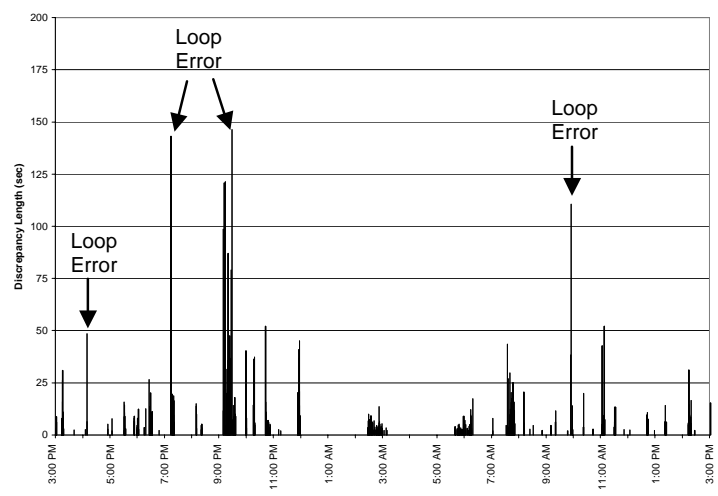

d) System 2 L1V0

Figure 6-25: Phase 1, Blanking Band = 2 sec., September $26 \& 27,2001$, Mostly Sunny, New Cameras 


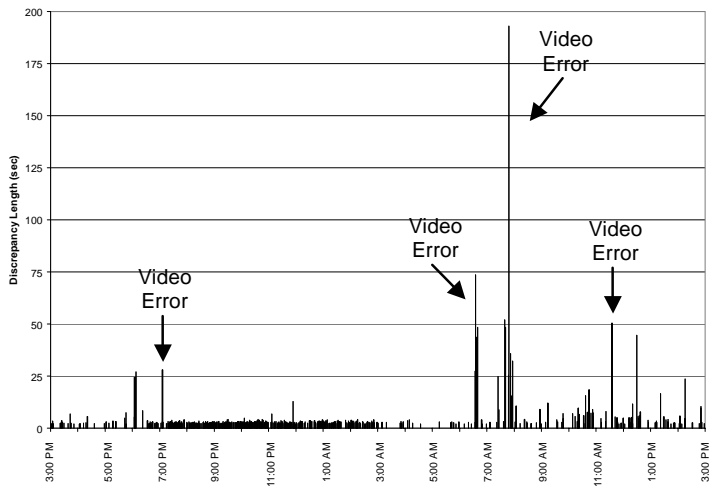

a) System 1 LOV1

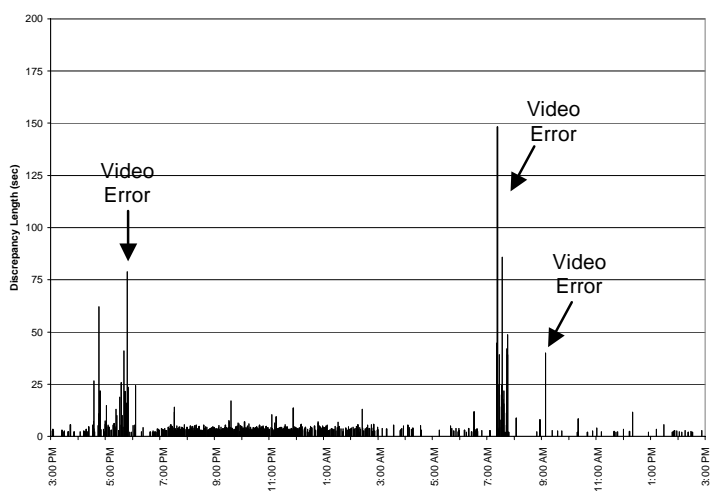

c) System 2 LOV1

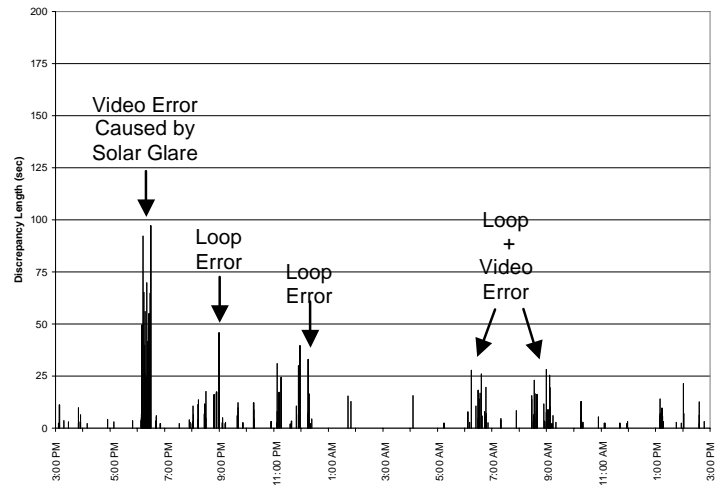

b) System 1 L1V0

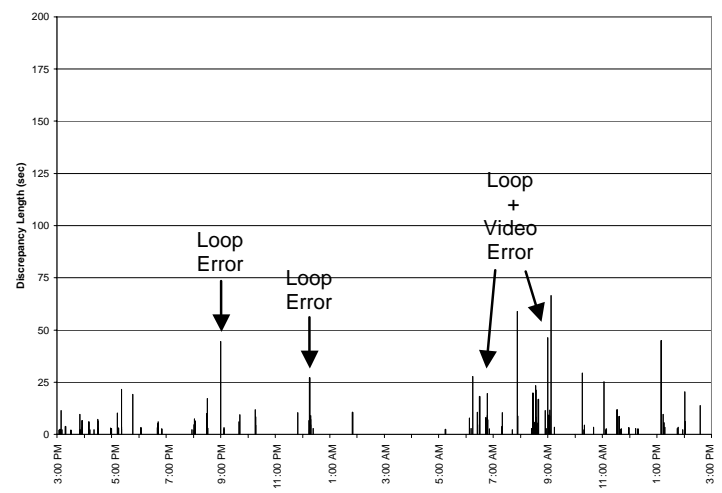

d) System 2 L1V0

Figure 6-26: Phase 3, Blanking Band = 2 sec., September 26 \& 27, 2001, Mostly Sunny, New Cameras 


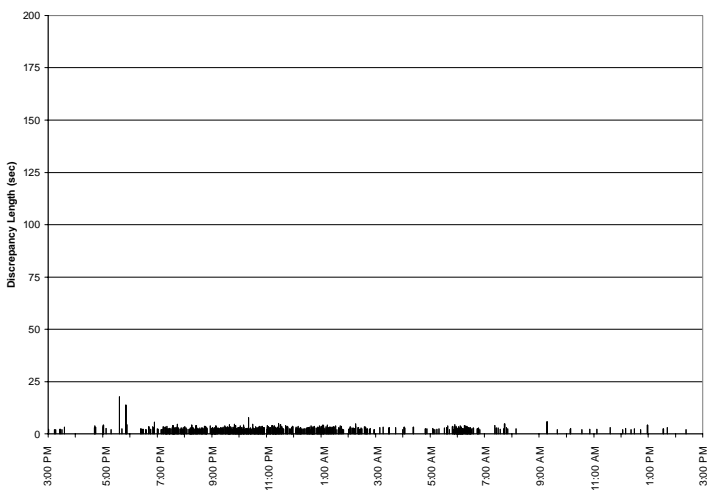

a) System 1 LOV1

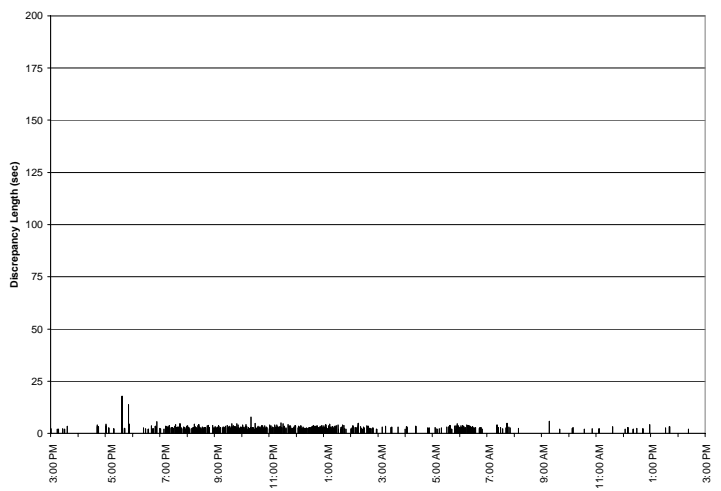

c) System 2 LOV1

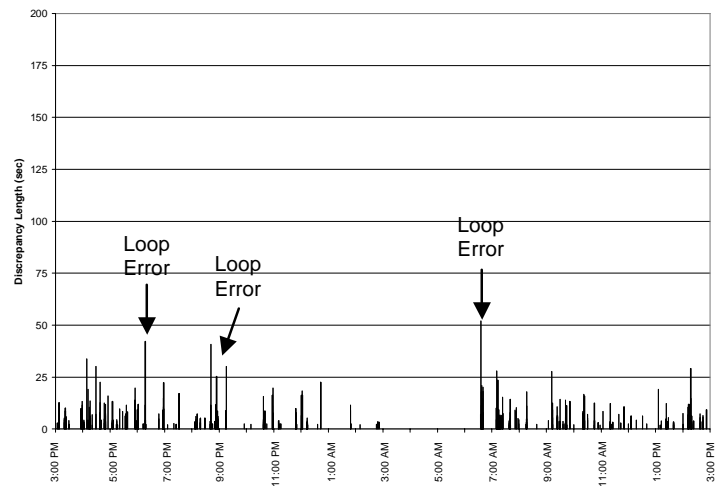

b) System 1 L1V0

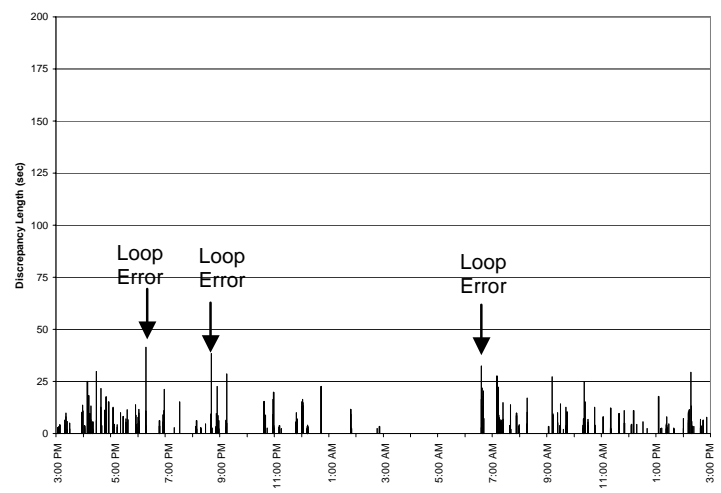

d) System 2 L1V0

Figure 6-27: Phase 6, Blanking Band = 2 sec., September $26 \&$ 27, 2001, Mostly Sunny, New Cameras 


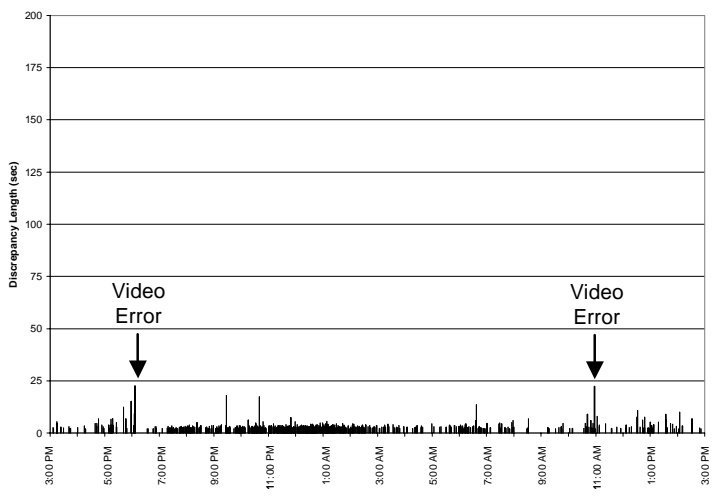

a) System 1 LOV1

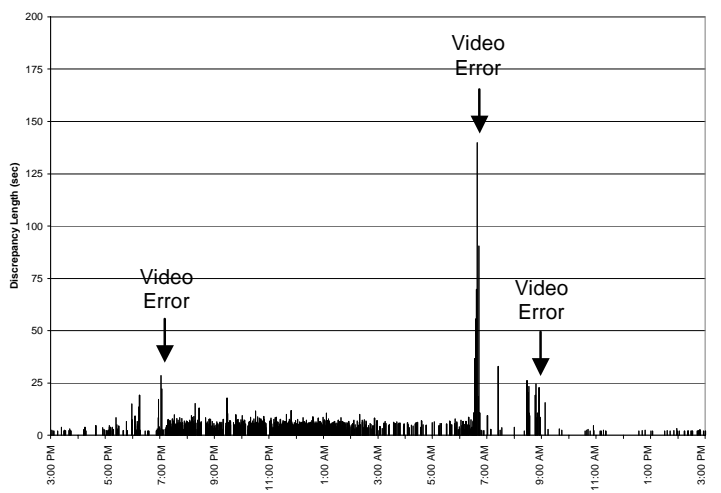

c) System 2 LOV1

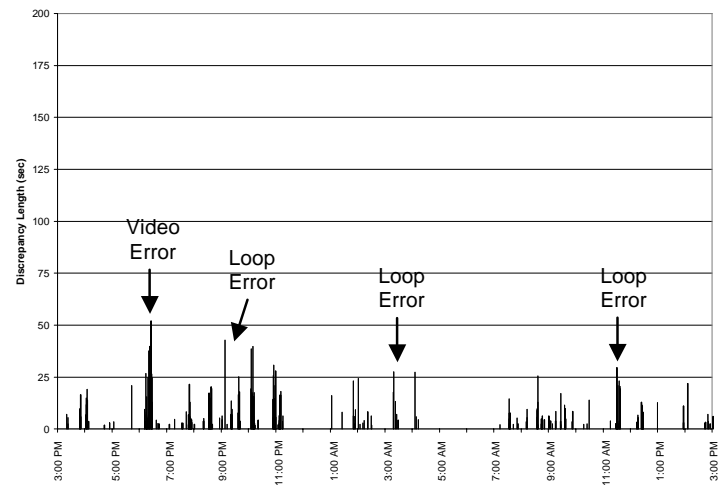

b) System 1 L1V0

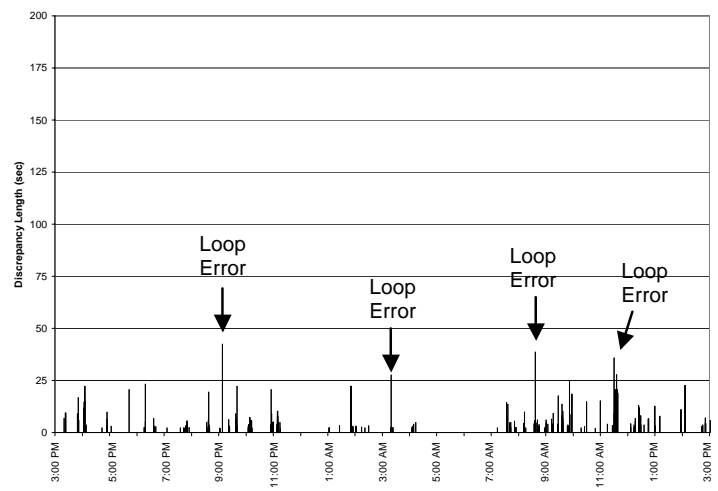

d) System 2 L1V0

Figure 6-28: Phase 8, Blanking Band = 2 sec., September 26 \& 27, 2001, Mostly Sunny, New Cameras 
Table 6-27: Hourly Discrepancy Totals for Phase 1, Blanking Band = $2 \mathrm{sec}$, September 26 \& 27, 2001, Mostly Sunny, New Cameras

\begin{tabular}{|r|r|r|r|r|}
\cline { 2 - 5 } \multicolumn{1}{c|}{} & \multicolumn{2}{c|}{ System 1 } & \multicolumn{2}{c|}{ System 2 } \\
\hline \hline Hour Beginning & L0V1 & L1V0 & L0V1 & L1V0 \\
\hline \hline 3:00 PM & 78 & 188 & 138 & 198 \\
\hline $4: 00 \mathrm{PM}$ & 432 & 57 & 133 & 65 \\
\hline $5: 00 \mathrm{PM}$ & 361 & 103 & 198 & 115 \\
\hline $6: 00 \mathrm{PM}$ & 68 & 305 & 53 & 304 \\
\hline $7: 00 \mathrm{PM}$ & 61 & 338 & 77 & 328 \\
\hline 8:00 PM & 202 & 72 & 27 & 57 \\
\hline 9:00 PM & 256 & 1236 & 15 & 1219 \\
\hline $10: 00 \mathrm{PM}$ & 10 & 375 & 14 & 366 \\
\hline $11: 00 \mathrm{PM}$ & 13 & 263 & 20 & 265 \\
\hline $12: 00 \mathrm{AM}$ & 0 & 0 & 0 & 0 \\
\hline $1: 00 \mathrm{AM}$ & 0 & 0 & 0 & 0 \\
\hline $2: 00 \mathrm{AM}$ & 0 & 199 & 2 & 199 \\
\hline $3: 00 \mathrm{AM}$ & 0 & 10 & 0 & 10 \\
\hline $4: 00 \mathrm{AM}$ & 0 & 0 & 4 & 0 \\
\hline $5: 00 \mathrm{AM}$ & 2 & 94 & 3 & 94 \\
\hline $6: 00 \mathrm{AM}$ & 191 & 113 & 1381 & 110 \\
\hline $7: 00 \mathrm{AM}$ & 349 & 317 & 117 & 420 \\
\hline $8: 00 \mathrm{AM}$ & 165 & 41 & 750 & 38 \\
\hline $9: 00 \mathrm{AM}$ & 2 & 197 & 5 & 197 \\
\hline $10: 00 \mathrm{AM}$ & 65 & 3 & 749 & 26 \\
\hline $11: 00 \mathrm{AM}$ & 173 & 204 & 80 & 202 \\
\hline $12: 00 \mathrm{PM}$ & 29 & 40 & 41 & 40 \\
\hline $1: 00 \mathrm{PM}$ & 34 & 37 & 33 & 37 \\
\hline $2: 00 \mathrm{PM}$ & 44 & 213 & 49 & 190 \\
\hline \hline Total & 2535 & 4405 & 3888 & 4480 \\
\hline & & & & \\
\hline
\end{tabular}


Table 6-28: Hourly Discrepancy Totals for Phase 3, Blanking Band = $2 \mathrm{sec}$, September 26 \& 27, 2001, Mostly Sunny, New Cameras

\begin{tabular}{|c|c|c|c|c|}
\hline & \multicolumn{2}{|c|}{ System 1} & \multicolumn{2}{|c|}{ System 2} \\
\hline Hour Beginning & LoV1 & L1V0 & LoV1 & L1V0 \\
\hline 3:00 PM & $\overline{33}$ & 42 & 31 & 45 \\
\hline 4:00 PM & 19 & 6 & 207 & 46 \\
\hline 5:00 PM & 26 & 7 & 392 & 60 \\
\hline 6:00 PM & 132 & 969 & 73 & 23 \\
\hline 7:00 PM & 130 & 9 & 243 & 2 \\
\hline 8:00 PM & 118 & 194 & 214 & 85 \\
\hline 9:00 PM & 140 & 58 & 262 & 35 \\
\hline 10:00 PM & 140 & 28 & 246 & 24 \\
\hline 11:00 PM & 136 & 175 & 244 & 71 \\
\hline $12: 00 \mathrm{AM}$ & 112 & 78 & 181 & 66 \\
\hline 1:00 AM & 82 & 28 & 136 & 11 \\
\hline $2: 00$ AM & 80 & 0 & 124 & 0 \\
\hline 3:00 AM & 22 & $\overline{0}$ & 33 & 0 \\
\hline 4:00 AM & 12 & 16 & 25 & $\overline{0}$ \\
\hline 5:00 AM & 22 & 2 & 31 & 2 \\
\hline $6: 00 \mathrm{AM}$ & 214 & 182 & 47 & 118 \\
\hline 7:00 AM & 459 & 17 & 684 & 84 \\
\hline $8: 00$ AM & 44 & 166 & 19 & 176 \\
\hline 9:00 AM & 39 & 113 & 48 & 110 \\
\hline $10: 00 \mathrm{AM}$ & 124 & 34 & 15 & 39 \\
\hline $11: 00 \mathrm{AM}$ & 95 & 12 & 16 & 58 \\
\hline 12:00 PM & 114 & 0 & 16 & 5 \\
\hline 1:00 PM & 62 & 66 & 23 & 79 \\
\hline 2:00 PM & 71 & 52 & 19 & 40 \\
\hline Total & 2427 & 2254 & 3329 & 1121 \\
\hline
\end{tabular}


Table 6-29: Hourly Discrepancy Totals for Phase 6, Blanking Band = $2 \mathrm{sec}$, September 26 \& 27, 2001, Mostly Sunny, New Camera

\begin{tabular}{|r|r|r|r|r|}
\cline { 2 - 5 } \multicolumn{1}{c|}{} & \multicolumn{2}{c|}{ System 1 } & \multicolumn{2}{c|}{ System 2 } \\
\hline \hline Hour Beginning & \multicolumn{1}{c|}{ L0V1 } & L1V0 & L0V1 & L1V0 \\
\hline \hline 3:00 PM & 14 & 135 & 36 & 127 \\
\hline $4: 00 \mathrm{PM}$ & 10 & 312 & 21 & 294 \\
\hline $5: 00 \mathrm{PM}$ & 55 & 177 & 59 & 160 \\
\hline $6: 00 \mathrm{PM}$ & 32 & 214 & 66 & 180 \\
\hline $7: 00 \mathrm{PM}$ & 131 & 33 & 340 & 18 \\
\hline 8:00 PM & 123 & 240 & 273 & 168 \\
\hline 9:00 PM & 174 & 51 & 390 & 43 \\
\hline $10: 00 \mathrm{PM}$ & 175 & 116 & 382 & 98 \\
\hline $11: 00 \mathrm{PM}$ & 159 & 58 & 381 & 50 \\
\hline $12: 00 \mathrm{AM}$ & 143 & 158 & 288 & 136 \\
\hline $1: 00 \mathrm{AM}$ & 99 & 26 & 204 & 26 \\
\hline $2: 00 \mathrm{AM}$ & 79 & 11 & 175 & 6 \\
\hline $3: 00 \mathrm{AM}$ & 17 & 0 & 67 & 0 \\
\hline $4: 00 \mathrm{AM}$ & 17 & 0 & 69 & 0 \\
\hline $5: 00 \mathrm{AM}$ & 69 & 0 & 192 & 0 \\
\hline $6: 00 \mathrm{AM}$ & 130 & 223 & 337 & 207 \\
\hline $7: 00 \mathrm{AM}$ & 25 & 322 & 20 & 279 \\
\hline $8: 00 \mathrm{AM}$ & 2 & 44 & 32 & 37 \\
\hline $9: 00 \mathrm{AM}$ & 8 & 197 & 25 & 147 \\
\hline $10: 00 \mathrm{AM}$ & 9 & 123 & 38 & 111 \\
\hline $11: 00 \mathrm{AM}$ & 5 & 64 & 23 & 56 \\
\hline $12: 00 \mathrm{PM}$ & 16 & 24 & 29 & 31 \\
\hline $1: 00 \mathrm{PM}$ & 6 & 75 & 29 & 60 \\
\hline $2: 00 \mathrm{PM}$ & 2 & 195 & 38 & 183 \\
\hline \hline Total & 1499 & 2796 & 3517 & 2414 \\
\hline & & & & \\
\hline
\end{tabular}


Table 6-30: Hourly Discrepancy Totals for Phase 8, Blanking Band = 2 sec., September 26 \& 27, 2001, Mostly Sunny, New Camera

\begin{tabular}{|c|r|r|r|r|}
\cline { 2 - 5 } \multicolumn{1}{c|}{} & \multicolumn{2}{c|}{ System 1 } & \multicolumn{2}{c|}{ System 2 } \\
\hline \hline Hour Beginning & \multicolumn{1}{c|}{ L0V1 } & \multicolumn{1}{c|}{ L0V1 } & L1V0 \\
\hline 3:00 PM & 40 & 56 & 29 & 55 \\
\hline $4: 00$ PM & 39 & 78 & 25 & 95 \\
\hline $5: 00$ PM & 102 & 24 & 91 & 24 \\
\hline $6: 00$ PM & 66 & 361 & 150 & 43 \\
\hline $7: 00$ PM & 75 & 106 & 374 & 35 \\
\hline 8:00 PM & 100 & 148 & 416 & 43 \\
\hline 9:00 PM & 121 & 179 & 451 & 107 \\
\hline 10:00 PM & 188 & 367 & 534 & 131 \\
\hline $11: 00$ PM & 227 & 116 & 592 & 47 \\
\hline $12: 00$ AM & 235 & 0 & 580 & 0 \\
\hline $1: 00$ AM & 196 & 71 & 443 & 43 \\
\hline $2: 00$ AM & 107 & 50 & 252 & 13 \\
\hline $3: 00$ AM & 45 & 70 & 89 & 34 \\
\hline $4: 00$ AM & 33 & 38 & 92 & 18 \\
\hline $5: 00$ AM & 47 & 0 & 103 & 0 \\
\hline $6: 00$ AM & 99 & 0 & 767 & 0 \\
\hline $7: 00$ AM & 51 & 59 & 56 & 74 \\
\hline 8:00 AM & 16 & 110 & 233 & 96 \\
\hline $9: 00$ AM & 27 & 112 & 24 & 177 \\
\hline $10: 00$ AM & 68 & 19 & 16 & 37 \\
\hline $11: 00$ AM & 26 & 169 & 9 & 181 \\
\hline $12: 00$ PM & 70 & 92 & 7 & 117 \\
\hline $1: 00$ PM & 58 & 14 & 18 & 27 \\
\hline $2: 00$ PM & 32 & 41 & 28 & 40 \\
\hline \hline Total & 2069 & 2281 & 5377 & 1437 \\
\hline
\end{tabular}

Table 6-31 gives the 24 hour totals of LOV1 and L1V0 discrepancies for System 1 and System 2 after the new System 2 cameras were installed on the southbound and eastbound approaches. By comparing the numbers shown in this table with those in Table 6-26, one can see that there has been very little, if any improvement in System 2 performance with the new cameras. One must be careful not to compare the totals, because in Table 6-26, the totals shown are for all eight phases, while in Table 6-31, the totals shown are only for four phases. What should be noted is the difference between the System 1 and System 2 discrepancies in Table 6-26, and the difference between the discrepancies in Table 6-31. There are some phases on which System 2 performance appears to 
be much better, but others on which it appears to have gotten worse. Therefore, it can be deduced that the special cameras have little effect upon the System 2 performance. It should be noted that there are specific times during the day when System 2 clearly outperforms System 1. These times are the day-to-night and night-to-day transitions. During these transitions, the sun commonly will cause so much glare in the camera that it is unable to distinguish the presence or absence of vehicles. The System 2 camera with the System 2 unit appears to handle these times somewhat better (although still not perfectly) than System 1.

Table 6-31: 24 Hour Discrepancy Totals (in seconds) After New System 2 Camera Installation on Eastbound and Southbound Approaches, Blanking Band $=2 \mathrm{sec}$.

\begin{tabular}{|c|c|c|c|c|c|}
\hline \multirow{3}{*}{\multicolumn{2}{|c|}{\begin{tabular}{|l} 
Data Collection Date \\
System \\
Discrepancy Type
\end{tabular}}} & \multicolumn{4}{|c|}{ September 26 \& 27, 2001} \\
\hline & & \multicolumn{2}{|c|}{ System 1} & \multicolumn{2}{|c|}{ System 2} \\
\hline & & LoV1 & L1V0 & LoV1 & L1V0 \\
\hline \multirow{4}{*}{ 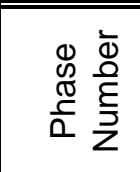 } & 1 & 2535 & 4405 & 3888 & 4480 \\
\hline & $\overline{3}$ & 2427 & 2254 & 3329 & $\overline{1121}$ \\
\hline & $\overline{6}$ & 1499 & 2796 & 3517 & 2414 \\
\hline & 8 & 2069 & 2281 & 5377 & 1437 \\
\hline \multicolumn{2}{|c|}{$\begin{array}{c}\text { Total (Southbound } \\
\text { and Eastbound Only) }\end{array}$} & 8530 & 11736 & 16111 & 9452 \\
\hline
\end{tabular}

\section{Calibration Effects}

One of the simplest ways to quantitatively show the effects of calibration on a video detection system is to show the effects of early headlight detection on an approach before the recalibration, and then after the recalibration. In order to do this, the distances from the stop bar on each approach were measured.

Figure 6-29 through Figure 6-32 show these distances on screen captures of the approaches. Two night videos, one before the recalibration, and one after the recalibration, were then watched to determine the distances at which the headlights of vehicles would trigger the video detection systems. The results of this activity are shown in Figure 6-33 and Figure 6-34. The ideal scenario would be to have a sharp peak at the same distance the inductive loop detectors are 
from the stop bar (approximately $50 \mathrm{ft}$ ), with no outliers. One can see that before the recalibration, the detection distances were further from the stop bar, and more spread out. After the recalibration, the detection distances moved closer to the stop bar, and the spread of the detections was less. It should be noted that the Autoscope 2004 unit has a feature called "Night Reflections." The purpose of this feature is to reduce the effect that vehicle headlight glare has on vehicle detection. This feature was not enabled before the recalibration, but was enabled during the recalibration.

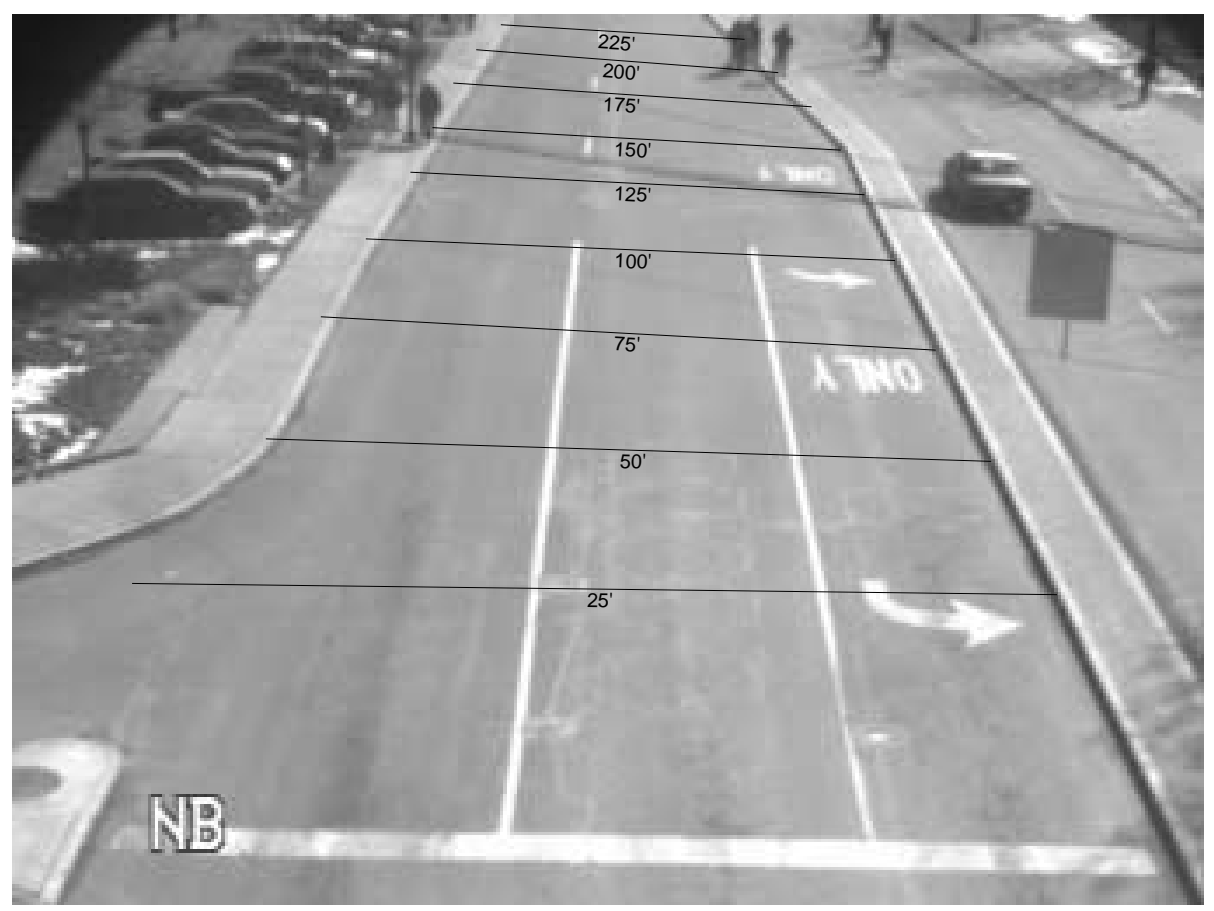

Figure 6-29: Distances from Stop Bar on Northbound Approach 


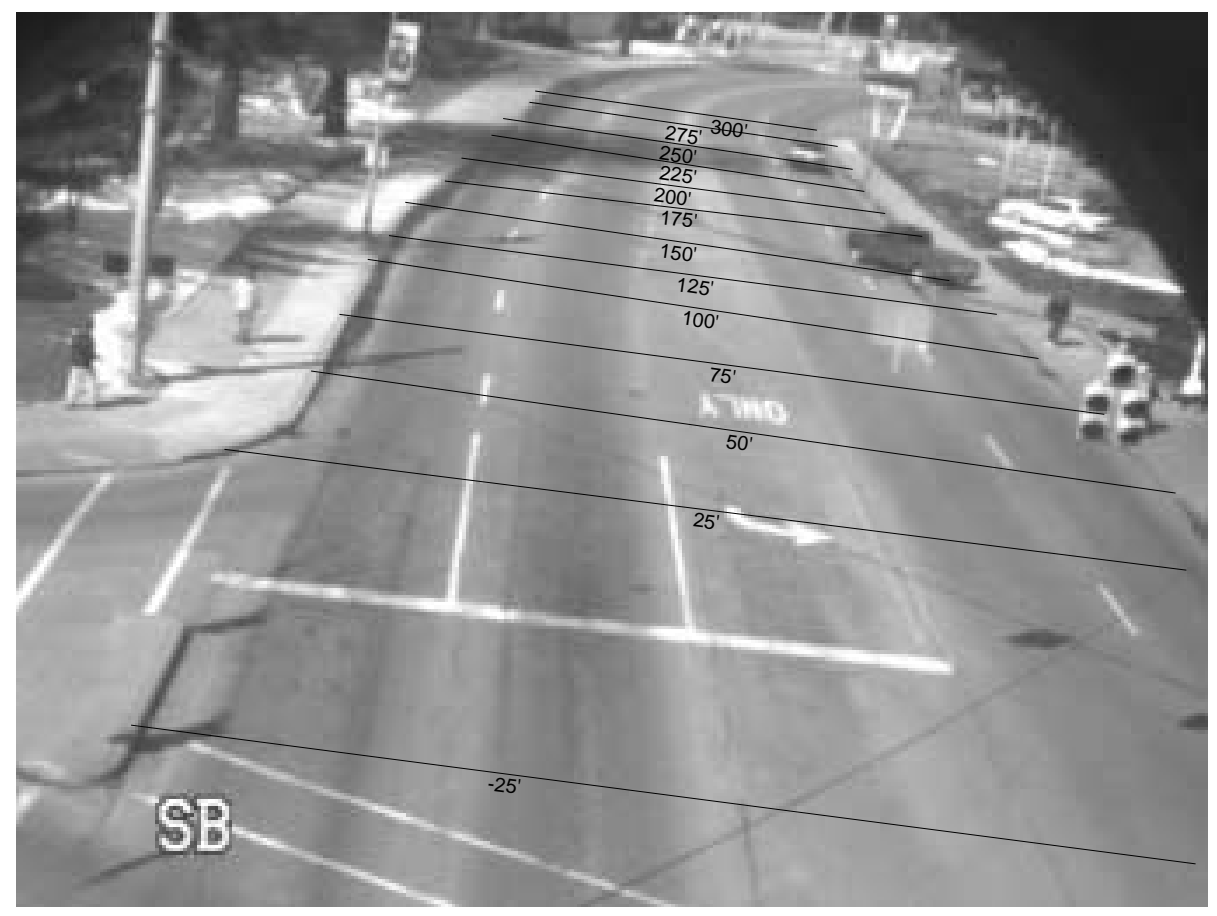

Figure 6-30: Distances from Stop Bar on Southbound Approach

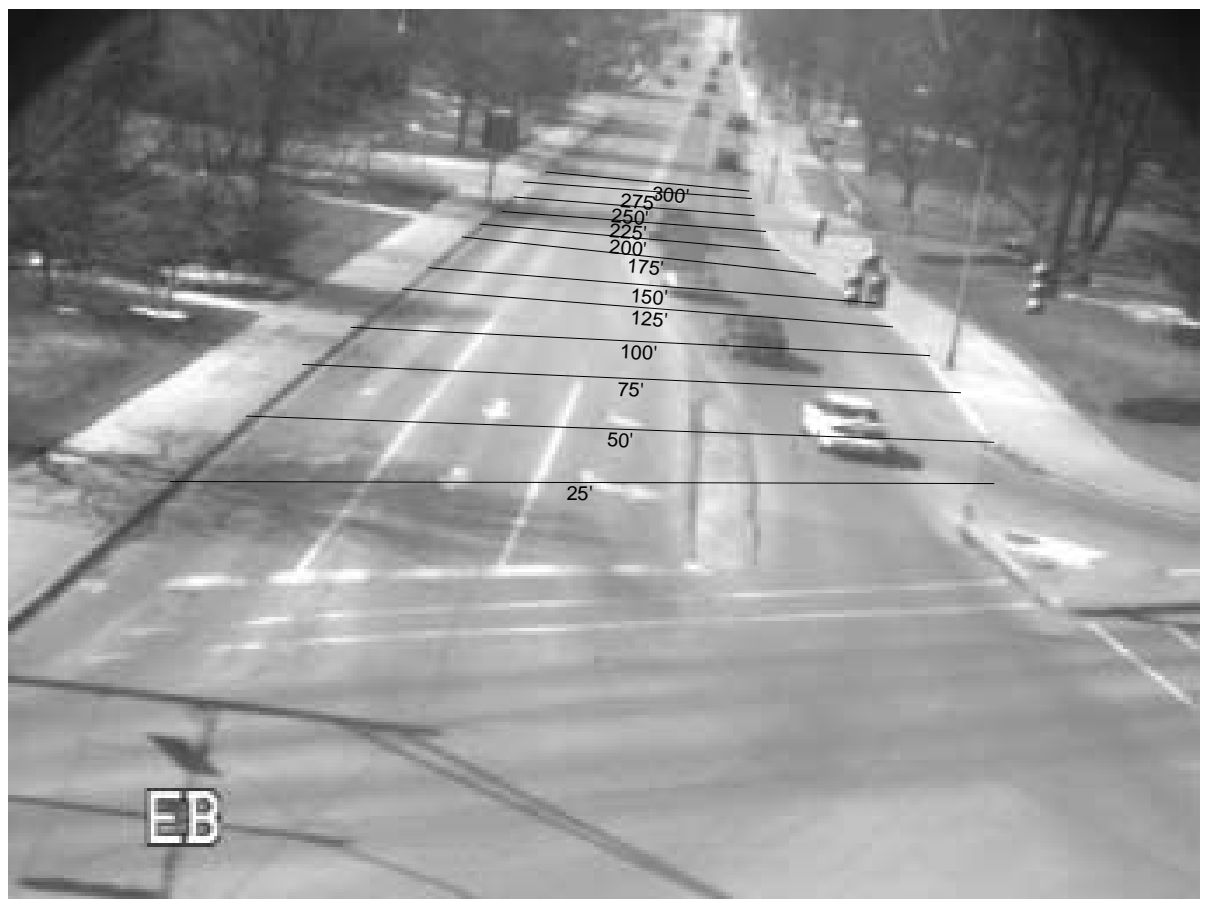

Figure 6-31: Distances from Stop Bar on Eastbound Approach 


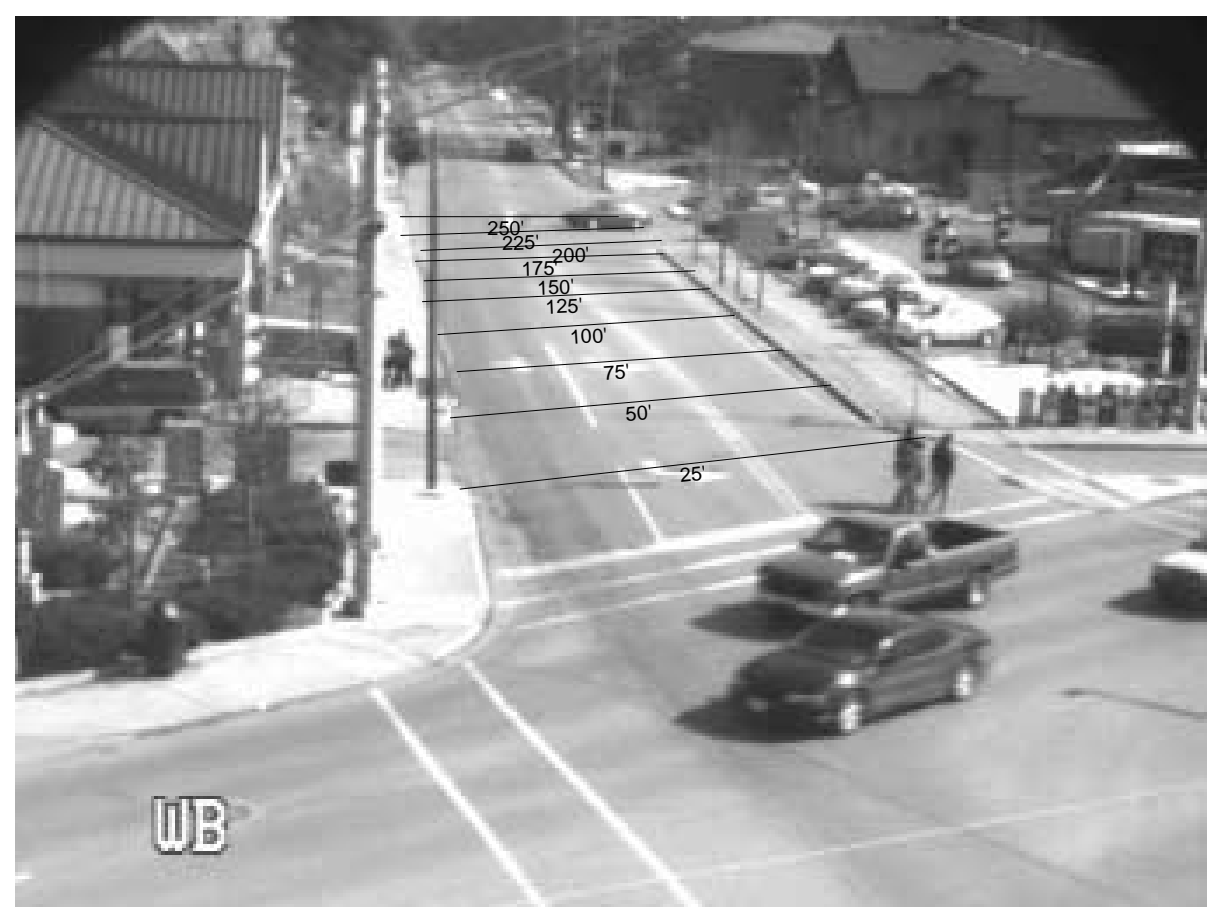

Figure 6-32: Distances from Stop Bar on Westbound Approach 


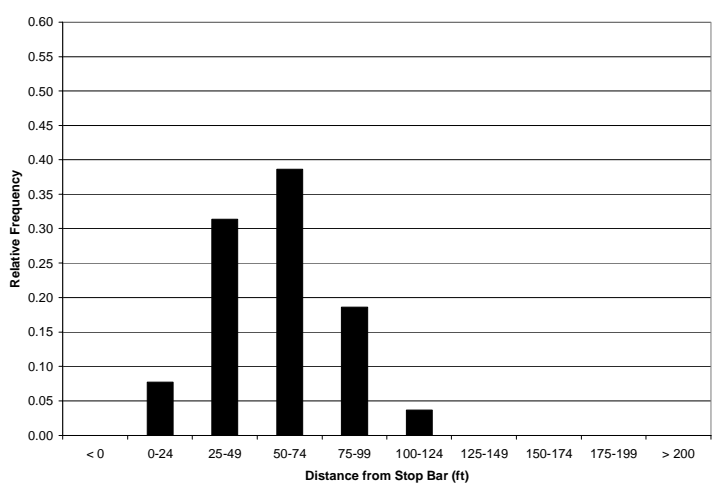

a) Northbound Approach, System 1 Nov. 7, 2000, 8:00 - 9:00 PM Sample Size $=220$

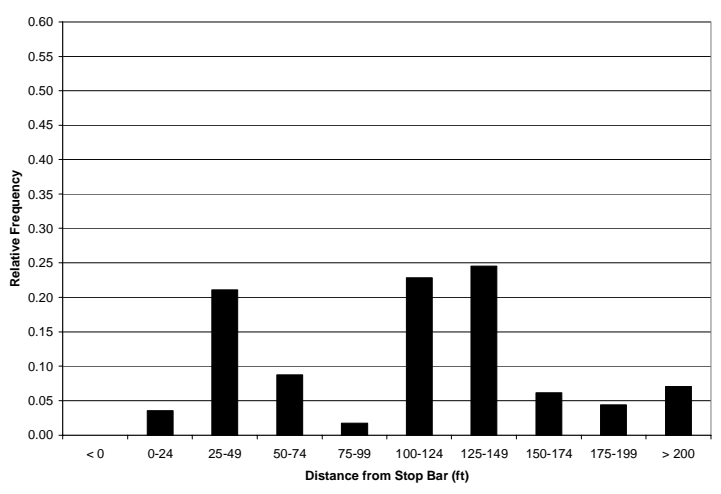

c) Southbound Approach, System 1 Nov. 2, 2000, 11:00 PM - 12:00 AM

Sample Size $=114$

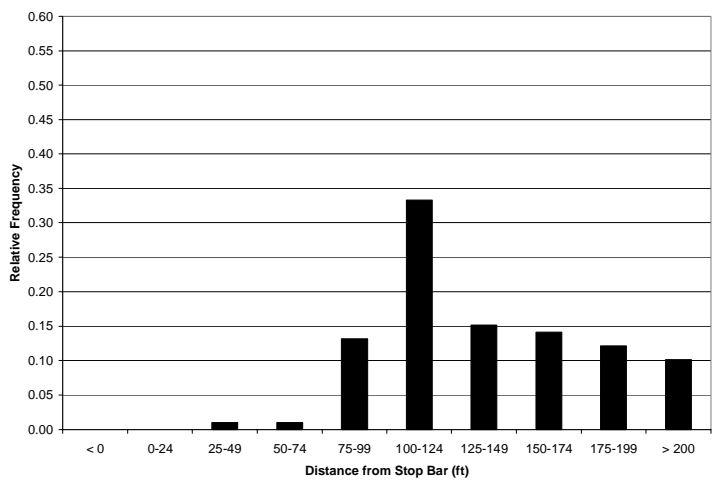

e) Eastbound Approach, System 1 Nov. 2, 2000, 11:00 PM - 12:00 AM Sample Size $=99$

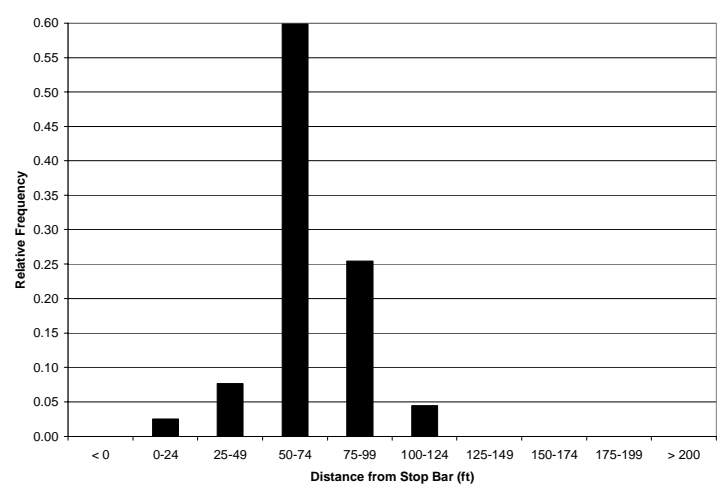

b) Northbound Approach, System 2 Nov. 7, 2000, 8:00 - 9:00 PM Sample Size $=157$

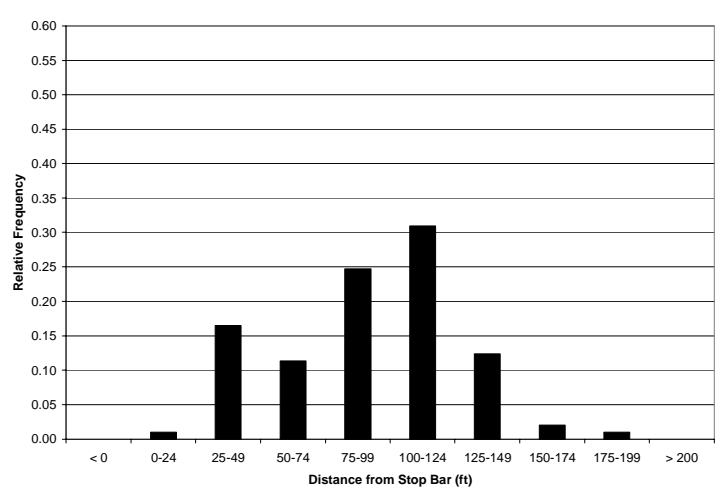

d) Southbound Approach, System 2 Nov. 2, 2000, 11:00 PM - 12:00 AM Sample Size $=97$

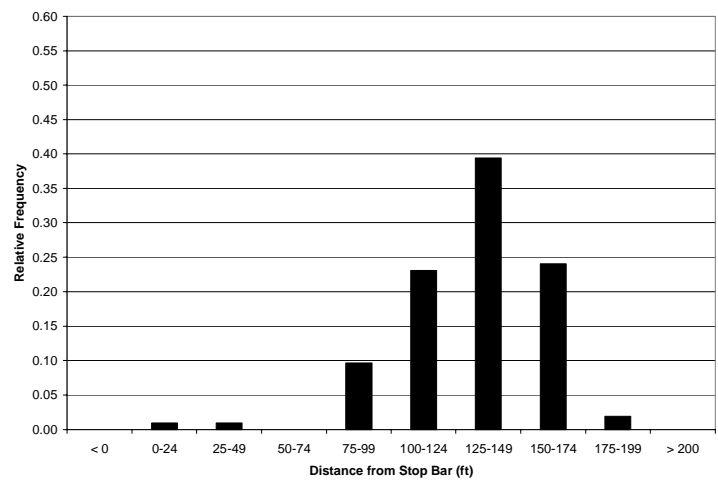

f) Eastbound Approach, System 2 Nov. 2, 2000, 11:00 PM - 12:00 AM Sample Size $=104$

Figure 6-33: Effect of Headlights on Early Video Detection at Night Before Recalibration 


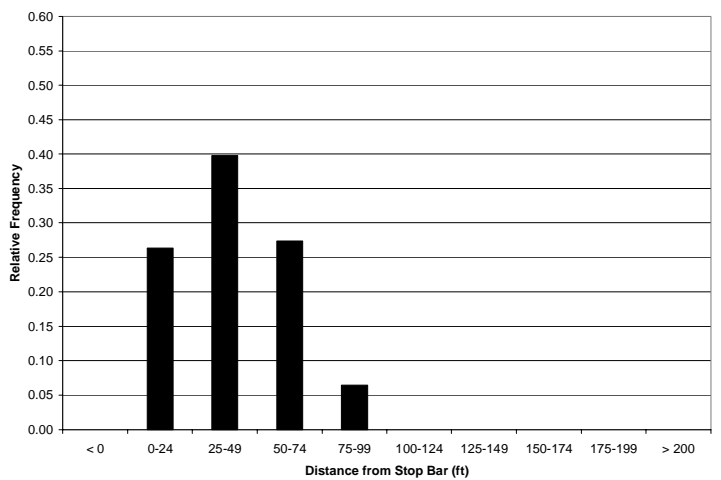

a) Northbound Approach, System 1 April 3, 2001, 8:00 - 9:00 PM Sample Size $=201$

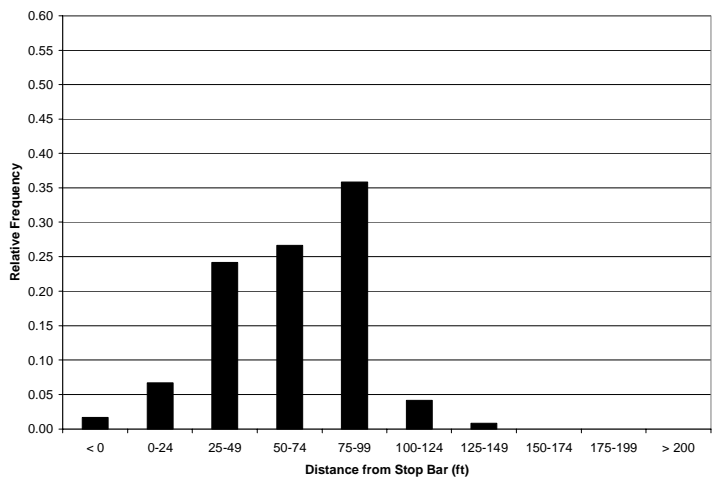

c) Southbound Approach, System 1 April 3, 2001, 9:00 - 10:00 PM Sample Size $=120$

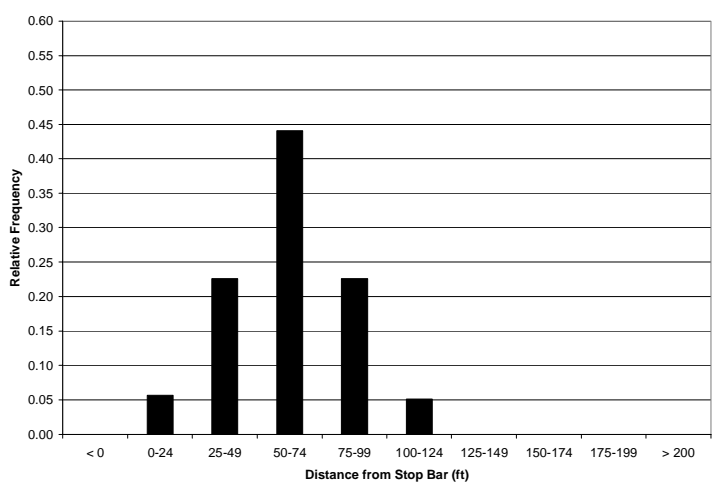

e) Eastbound Approach, System 1 April 3, 2001, 10:00 - 11:00 PM Sample Size $=195$

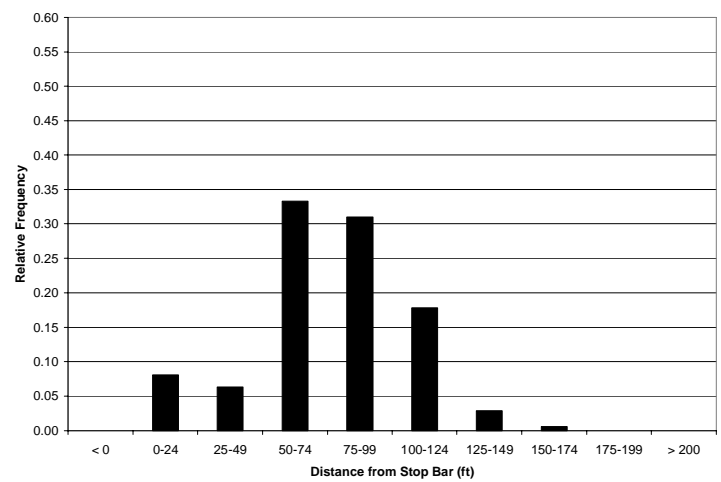

b) Northbound Approach, System 2 April 3, 2001, 8:00 - 9:00 PM Sample Size $=174$

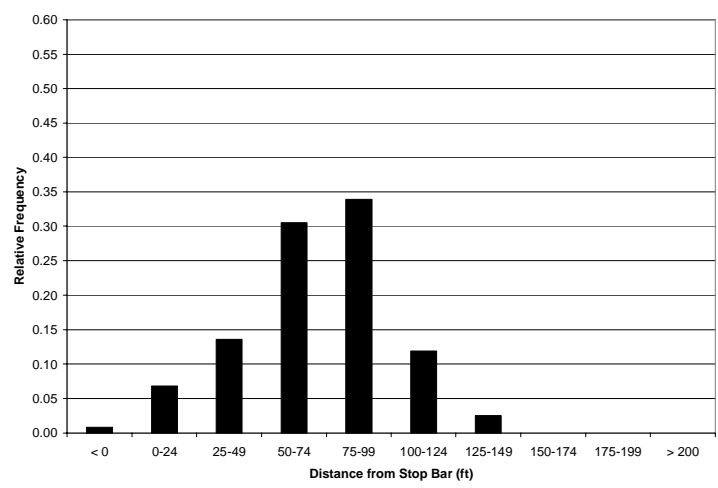

d) Southbound Approach, System 2 April 3, 2001, 9:00 - 10:00 PM Sample Size $=118$

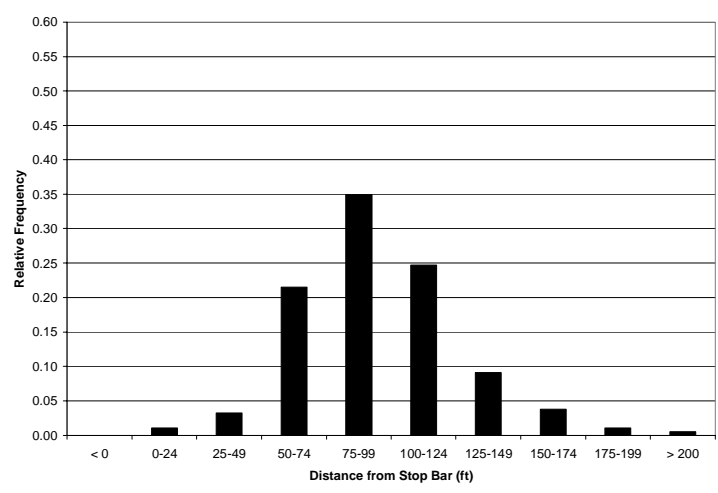

f) Eastbound Approach, System 2 April 3, 2001, 10:00 - 11:00 PM

Sample Size $=186$

Figure 6-34: Effect of Headlights on Early Video Detection at Night After Recalibration 
Figure 6-35 shows the video detection activation distances during the day. Because the actual vehicle is detected during the day, and not the headlights, the peak of detection is nearer the stop bar, and there is almost no spread in the detections. Table 6-32 gives a summarization of the average and standard deviation of video detection activation distances at night before and after recalibration, and during the day after recalibration. One can see that the recalibration did positively affect both video detection systems on all three approaches, except for System 2 on the Northbound approach, which was only slightly worse than its pre-calibration state. 


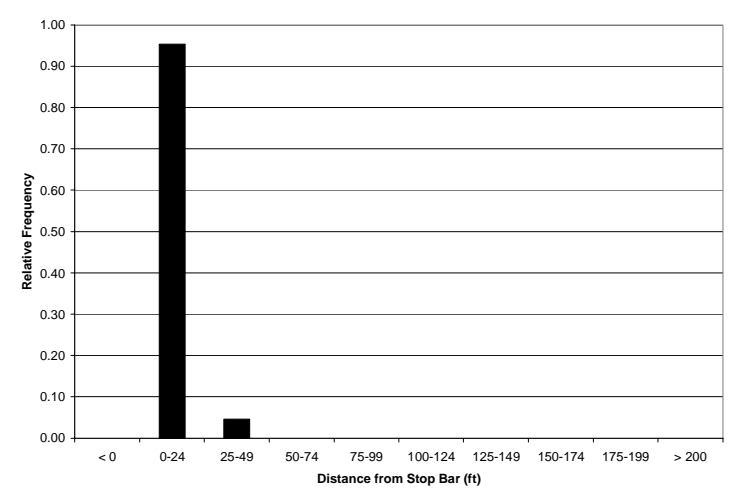

a) Northbound Approach, System 1 April 29, 2001, 8:00 AM - 9:00 AM

Sample Size $=108$

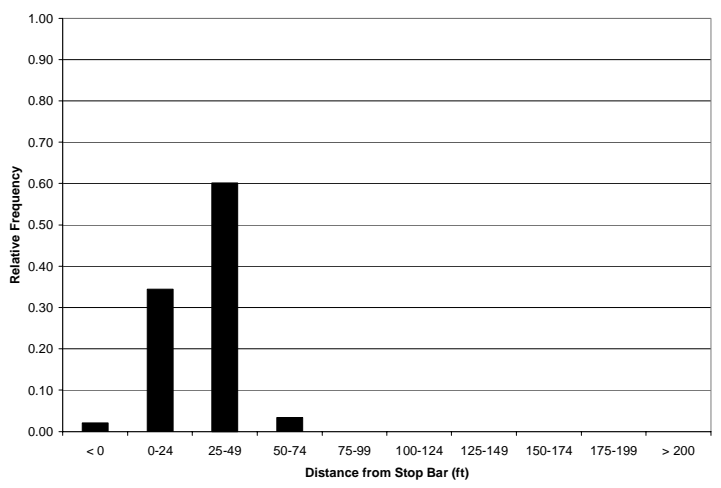

c) Southbound Approach, System 1 April 29, 2001, 9:00 AM - 10:00 AM Sample Size $=148$

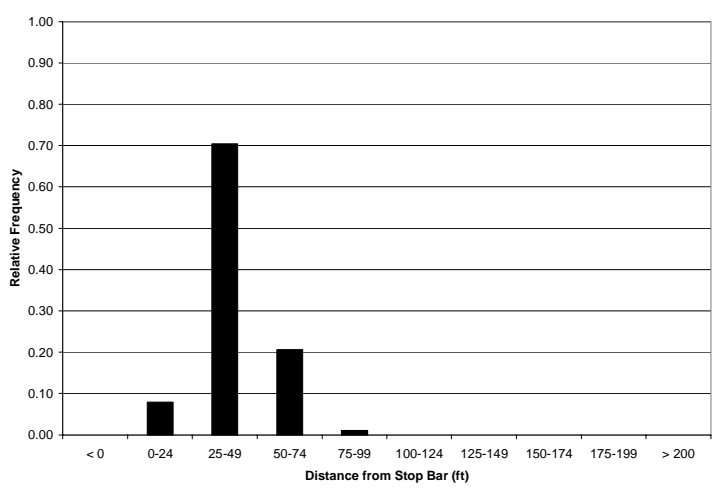

e) Eastbound Approach, System 1 April 29, 2001, 10:07 AM - 11:07 AM Sample Size $=189$

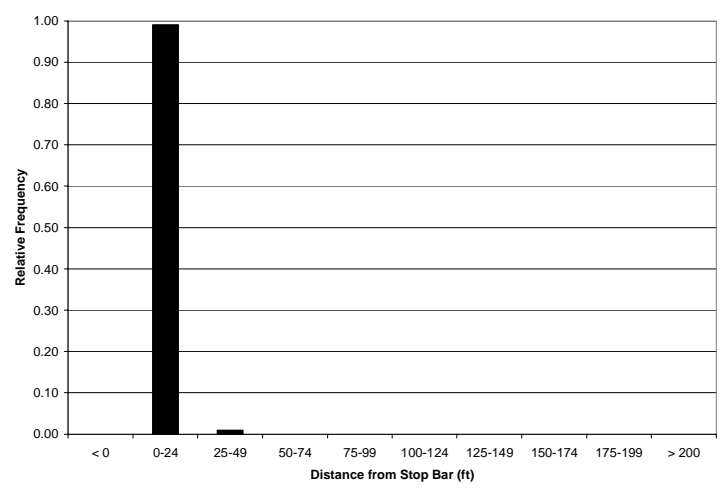

b) Northbound Approach, System 2 April 29, 2001, 8:00 AM - 9:00 AM Sample Size $=107$

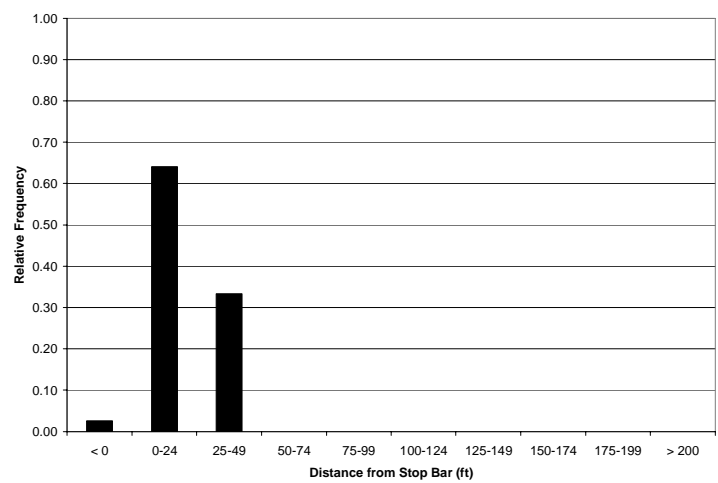

d) Southbound Approach, System 2 April 29, 2001, 9:00 AM - 10:00 AM Sample Size $=156$

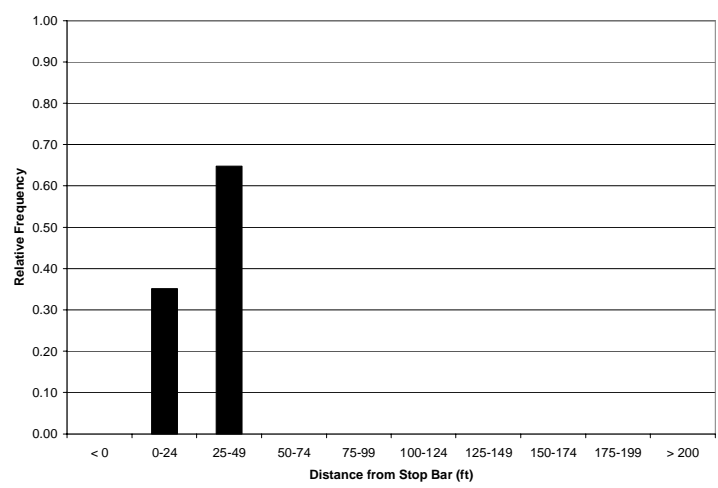

f) Eastbound Approach, System 2 April 29, 2001, 10:07 AM - 11:07 AM Sample Size $=196$

Figure 6-35: Daytime Video Detection Activation Distances After Recalibration 
Table 6-32: Summarization of Video Detection Activation Distances (ft)

\begin{tabular}{|c|r|r|r|r|r|r|}
\cline { 2 - 7 } \multicolumn{1}{c|}{} & \multicolumn{2}{c|}{$\begin{array}{c}\text { Before Recalibration - } \\
\text { Night }\end{array}$} & \multicolumn{2}{c|}{$\begin{array}{c}\text { After Recalibration - } \\
\text { Night }\end{array}$} & \multicolumn{2}{c|}{$\begin{array}{c}\text { After Recalibration - } \\
\text { Day }\end{array}$} \\
\hline System/Approach & $\begin{array}{c}\text { Weighted } \\
\text { Average }\end{array}$ & $\begin{array}{c}\text { Standard } \\
\text { Deviation }\end{array}$ & $\begin{array}{c}\text { Weighted } \\
\text { Average }\end{array}$ & $\begin{array}{c}\text { Standard } \\
\text { Deviation }\end{array}$ & $\begin{array}{c}\text { Weighted } \\
\text { Average }\end{array}$ & $\begin{array}{c}\text { Standard } \\
\text { Deviation }\end{array}$ \\
\hline \hline System 1, Northbound & 56.8 & 23.9 & 40.5 & 22.1 & 13.2 & 5.3 \\
\hline System 2, Northbound & 67.7 & 18.9 & 75.8 & 30.2 & 12.2 & 2.4 \\
\hline System 1, Southbound & 107.4 & 55.0 & 63.0 & 28.1 & 28.2 & 14.5 \\
\hline System 2, Southbound & 91.6 & 35.4 & 70.9 & 29.7 & 19.7 & 12.9 \\
\hline System 1, Eastbound & 137.5 & 40.9 & 61.7 & 23.5 & 40.7 & 13.9 \\
\hline System 2, Eastbound & 131.2 & 28.4 & 94.5 & 31.8 & 28.2 & 12.0 \\
\hline
\end{tabular}

It should be noted here that the method used for computing the distances reported in Table 6-32 was a weighted average. When looking at Figure 6-33, Figure 6-34, and Figure 6-35, one can see that the distances were reported in bins. The average of the bin edges was used in order to determine the average activation distance. For example, if the bin was $25 \mathrm{ft}$ - $49 \mathrm{ft}$., the average of these two numbers, $37 \mathrm{ft}$. was used for the distance in the calculations. This is what causes the average activation distances during the day to seem so small. They are actually more toward the back edge of the bin than they are toward the front, but because this weighted average is used in the calculations, the resulting average activation distances are somewhat smaller than they actually are.

Table 6-33 shows the absolute and percent increase in the video detection activation distance at night with respect to the daytime values. The smallest percent increase in the effective detector length was over $50 \%$. The largest was upwards of $500 \%$. It is obvious that nighttime conditions have a dramatic effect on the effective video detector length, and can thus cause an intersection to operate inefficiently, especially on shorter winter days when the sun rises later and sets earlier. During these times, it is possible for the peak periods to be partially during the night conditions. This table also shows that night conditions have a greater effect on System 2 activation distances than they do on System 1. 
In fact, System 2 appears to be more than twice as sensitive to headlights than System 1.

Table 6-33: Increase in Video Detection Activation Distances from Day to Night Due to Headlights

\begin{tabular}{|r|r|r|r|r|}
\cline { 2 - 5 } \multicolumn{1}{c|}{} & \multicolumn{2}{c|}{ System 1 } & \multicolumn{2}{c|}{ System 2 } \\
\hline Approach & $\begin{array}{c}\text { Absolute Increase } \\
\text { (ft) }\end{array}$ & Percent Increase & $\begin{array}{c}\text { Absolute Increase } \\
\text { (ft) }\end{array}$ & Percent Increase \\
\hline \hline Northbound & 27.3 & $207.68 \%$ & 63.6 & $519.55 \%$ \\
\hline Southbound & 34.8 & $123.42 \%$ & 51.2 & $260.03 \%$ \\
\hline Eastbound & 21.0 & $51.69 \%$ & 66.3 & $235.21 \%$ \\
\hline
\end{tabular}

The effective detector length has a significant effect upon control of an actuated intersection. In fact, the longer the detector length, the longer the gap time that is provided, whether desirable or not. Table 6-34 (Orcutt 1993) shows quantitatively the effect of changing the detector length and approach speeds on the provided gap time. So, for example, in Figure 6-33e and Figure 6-33f, and assuming a vehicle approach speed of $30 \mathrm{mph}$, both systems erroneously introduced over two seconds of additional detection time for the majority of vehicles on the Eastbound approach.

Table 6-34: Allowed Gap (seconds) Provided by Various Loop Length/Approach Speed Combinations (Orcutt 1993)

\begin{tabular}{|c|r|r|r|r|r|r|r|r|r|}
\cline { 2 - 11 } \multicolumn{1}{c|}{} & \multicolumn{10}{c|}{ Loop Length $(\mathrm{ft})$} \\
\hline Approach Speed (mph) & \multicolumn{1}{c|}{20} & \multicolumn{1}{c|}{30} & \multicolumn{1}{c|}{50} & 50 & 60 & 70 & 80 & 90 & 100 \\
\hline \hline 20 & 0.7 & 1.0 & 1.4 & 1.7 & 2.0 & 2.4 & 2.7 & 3.1 & 3.4 \\
\hline 25 & 0.5 & 0.8 & 1.1 & 1.4 & 1.6 & 1.9 & 2.2 & 2.4 & 2.7 \\
\hline 30 & 0.5 & 0.7 & 0.9 & 1.1 & 1.4 & 1.6 & 1.8 & 2.0 & 2.3 \\
\hline 35 & 0.4 & 0.6 & 0.8 & 1.0 & 1.2 & 1.4 & 1.6 & 1.7 & 1.9 \\
\hline 40 & 0.3 & 0.5 & 0.7 & 0.9 & 1.0 & 1.2 & 1.4 & 1.5 & 1.7 \\
\hline 45 & 0.3 & 0.5 & 0.6 & 0.8 & 0.9 & 1.1 & 1.2 & 1.4 & 1.5 \\
\hline 50 & 0.3 & 0.4 & 0.5 & 0.7 & 0.8 & 1.0 & 1.1 & 1.2 & 1.4 \\
\hline 55 & 0.2 & 0.4 & 0.5 & 0.6 & 0.7 & 0.9 & 1.0 & 1.1 & 1.2 \\
\hline
\end{tabular}




\section{Concluding Remarks}

This evaluation effort has shown that it is possible to view the performance of video detection systems by determining the discrepancy between inductive loops and the video detectors. By plotting the individual L0V1 and L1V0 discrepancies on a graph, one can tell at what times there are major problems with the video detectors. For example, see the large peak in Figure 6-1c. By capturing the video with a text overlay, the time periods where there are major problems can then be viewed to determine the source of the problem, as in Figure 4-2 through Figure 4-7. This would be helpful in both training technicians how to set up video detection zones most effectively, and to aid in offline video detection calibration. Of the days picked for this evaluation, there was no weather condition during the day that was worse or better than others. It was shown; however, that the rain at night caused the magnitude of the LOV1 discrepancies to rise due to the higher amount of reflection of headlights off the pavement for both systems (see Table 6-1 through Table 6-8). System 2 tended to have less problems with detecting shadows in adjacent lanes (Figure 4-4), but overall, had more of a problem with both types of discrepancies before and after recalibration (Table 6-25 and Table 6-26), and with early headlight detection at night (Table 6-33). It was also shown that video detection system 2 did not improve drastically when System 2 cameras were used instead of System 1 camera (see Table 6-26 and Table 6-31). Lastly, it was shown that the video detectors have a longer effective detection length at night (Table 6-33), and thereby artificially increase the provided gap time, which causes a decrease in the efficiency of the intersection. 


\section{CHAPTER 7 - ANALYSIS OF LIKELIHOODS}

\section{Chapter Overview}

The purpose of this chapter is to estimate various types of MOEs for video detection that use conditional likelihoods. By performing a likelihood analysis of video detection performance, one can incorporate imperfection of loop detectors and develop statistical models to estimate the effects of weather and traffic conditions.

In the first section of this chapter, the performance of video detection is modeled with the assumption that inductive loops give perfect information. With this assumption, the discrepancy values can be used to evaluate video detection. In the second section, the performance of inductive loop detectors is modeled in order to test the assumption of perfect loops. The third section models the performance of video detection, while taking into account loop imperfection. The fourth section gives a sensitivity analysis of the derived model to show the effect of various weather and traffic conditions on the performance of video detection. The last section summarizes this chapter. 


\section{Discrepancies Between Video Detection and Loop Detection}

The objective of this section is to evaluate the performance of video detection compared to loop detection. The main focus in this section is on the quantification of weather and traffic effects on video detection.

\section{Data Collection}

To decide what data to collect, weather and traffic characteristics that may affect video and loop detection performance were determined as listed in Table 7-1. The list includes potential performance factors indicated in literature (MacCarley, et al. 1992, MacCarley 1998, Middleton, et al. 1999), or those selected based on the author's own expectations.

Table 7-1: Characteristics That Possibly Effect Video and Loop Detection Performance

\begin{tabular}{|c|c|}
\hline Characteristics & Descriptors of Characteristic \\
\hline \hline \multirow{2}{*}{ Precipitation } & None \\
& Rain \\
& Snow \\
\hline \multirow{2}{*}{ Pavement } & Dry \\
& Wet \\
\hline \multirow{2}{*}{ Camera Motion } & Snow Covered \\
& None/Low \\
& Moderate \\
& Heavy \\
\hline \multirow{2}{*}{ Lighting Conditions } & Overcast \\
& Sun \\
\hline Volume & Night \\
\hline \multirow{2}{*}{ Movement Type } & 15 minute count \\
& Left Turn \\
& Through \\
\hline
\end{tabular}

The data was collected using the test facility described in Chapter 3. Data was collected in ten periods ranging from 16 to 25 hours apiece. Inductive loop 
and video detector output and a video with text overlay were collected in each period. The resulting data was processed with the software described in Chapter 5 in order to calculate the likelihoods of discrepancy. An aggregation interval of 15 minutes was used to calculate the likelihoods and to aggregate all other pieces of data. The vehicle counts were determined by opening the database table of the upstream inductive loop detector in each lane and counting the number of times that it was activated.

After finding the likelihoods of discrepancy and the vehicle counts, the video was consulted in order to determine the lighting conditions, camera motion, and precipitation during the data collection periods. In order to do this, an observer scrolled through the digital RealVideo clips that show all four approaches of the intersection on the same screen (Figure 4-3). The digital video could be easily fast-forwarded, and thus it was necessary to only watch approximately 15 seconds (or until the above mentioned characteristics could confidently be assessed) of video for each fifteen-minute data aggregation interval. All weather characteristics were noted in a spreadsheet, which had one row per each fifteen-minute observation. Fifteen minutes of data for one traffic phase (movement) was considered to be one observation. For each observation, the characteristics in Table 7-1 were collected. For each fifteen-minute interval, eight observations can be made - one for each of the eight phases.

Once the weather conditions had been extracted from the video, a sample of nearly 660 observations was assembled. For each observation, the following data was available: precipitation, pavement condition, camera motion, lighting conditions, vehicle counts, vehicle movement type (left turn, through, etc.), and the conditional likelihoods of discrepancy between the video and inductive loop detectors. 
After all data had been collected, it was checked for completeness. All the characteristics in Table 7-1 are present in the data, except snow-covered pavement. Table 7-2 shows the number of observations of each weather characteristic and of each traffic movement included in the sample. The average fifteen-minute traffic count is also given.

Table 7-2: Conditions Represented in Sample

\begin{tabular}{|c|l|c|}
\hline \multicolumn{2}{|c|}{ Condition } & Number of Observations \\
\hline \hline \multirow{3}{*}{ Precipitation } & No Precipitation & 394 \\
\cline { 2 - 3 } & Rain & 155 \\
\cline { 2 - 3 } & Snow & 12 \\
\hline \multirow{2}{*}{ Pavement Surface } & Dry Pavement & 360 \\
\cline { 2 - 3 } & Wet Pavement & 201 \\
\hline \multirow{3}{*}{ Lighting Conditions } & Overcast & 235 \\
\cline { 2 - 3 } & Sun & 30 \\
\cline { 2 - 3 } & Night & 296 \\
\hline \multirow{3}{*}{ Camera Motion } & No/Low Camera Motion & 396 \\
\cline { 2 - 3 } & Moderate Camera Motion & 67 \\
\cline { 2 - 3 } & Heavy Camera Motion & 98 \\
\hline \multirow{3}{*}{ Movement Type } & Through Only & 95 \\
\cline { 2 - 3 } & Through/Right & 103 \\
\cline { 2 - 3 } & Left Turn & 363 \\
\hline
\end{tabular}

Note: The average vehicle count is 53.1 vehicles per fifteen minutes

\section{Preliminary Analysis}

In this section, the two conditional likelihoods of discrepancy between inductive loop detectors and video detectors, calculated with Equations 4-1 and 4-2, will be used.

The data was collected on March 4 - March 5, 2001, which was an overcast day. An overcast day provides the most optimal conditions for the video detection systems, because there are no shadows from vehicles to be falsely detected. A profile of likelihood values for false detections is presented in Figure 7-1. 


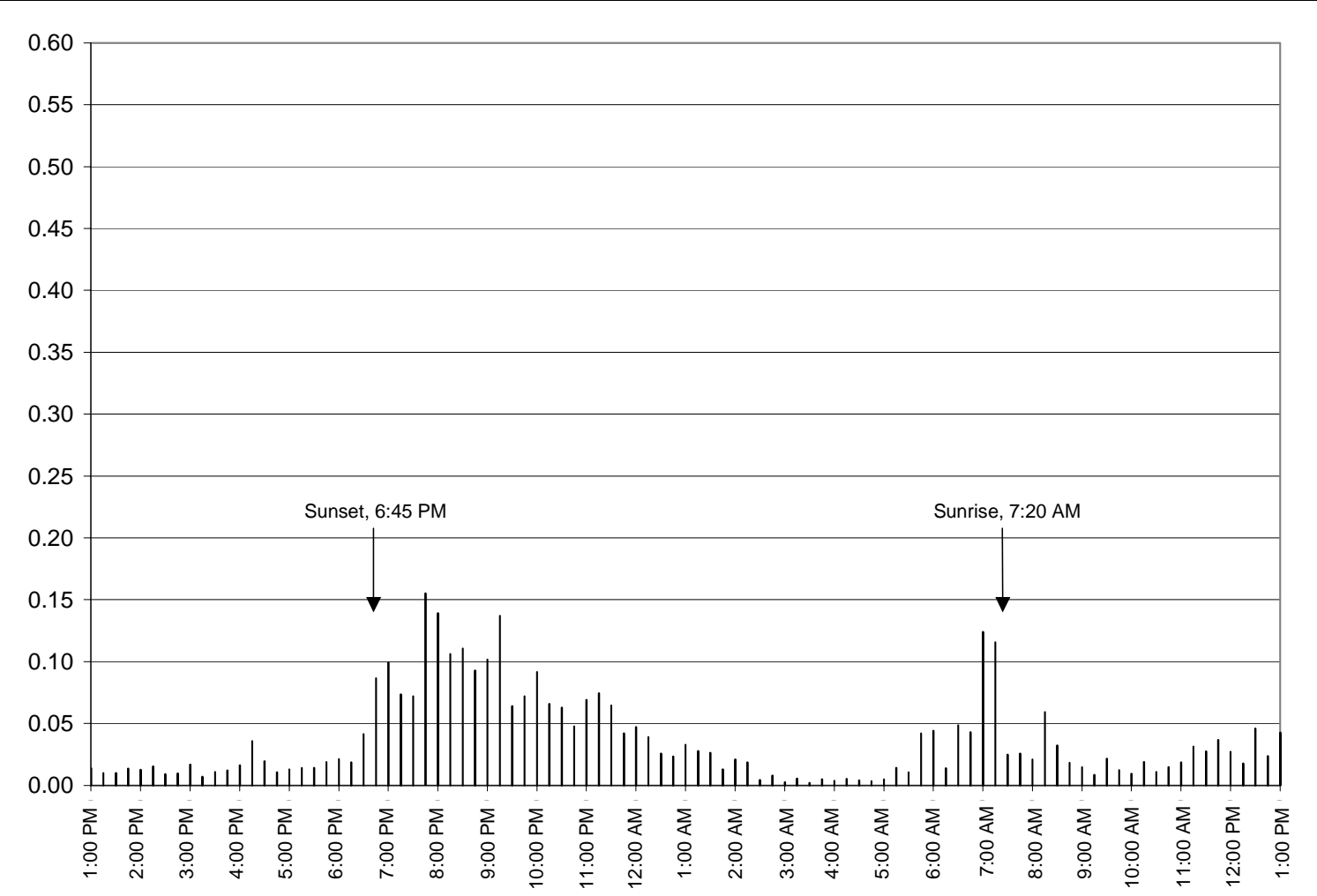

Figure 7-1: System 1 L0V1 Likelihood Profile (Northbound Left Turn Approach, March 4 \& 5, 2001, Overcast)

According to this figure, during the daylight hours there is approximately a 2 - $5 \%$ chance of having the LOV1 discrepancy. During the nighttime hours, however, the probability of this error fluctuates greatly. It is obvious that the combination of nighttime and high vehicle volume is associated with the highest likelihood of the LOV1 discrepancy. This trend is fully in accordance with the cause of the discrepancy explained in Chapter 6. This benefit comes from incorporating the opportunity for discrepancy to the measure. Around sunset and sunrise (the large peaks), there is a greater volume. During the night, each vehicle inherently brings with it a certain discrepancy due to the headlight beams. This is why the probability of a false or early detection rises so drastically during these times. The lower the volume gets (as in 1:00 AM-5:00 AM), the less frequent is headlight glare, and the lower the probability of early detections. This figure shows what one would expect. 
The likelihood estimates for the L1V0 discrepancy are shown in Figure 7-2. In calculating these likelihood estimates, the denominator is the amount of time that the inductive loop was on. During the early morning hours, this is a very small number due to the low volumes. To avoid a significant estimation error, any aggregation interval during which the inductive loops were on less than 20 seconds has been removed from the evaluation. These intervals are shown as black dots in Figure 7-2. In this figure, one may notice the peak between 12:00 AM and 6:00 AM. The increased L1V0 discrepancy is the result of the particular video detector layout. On this approach, the video detector zone does not extend past the stop bar. At night, the video detectors detect the headlights of vehicles and not the vehicles themselves. When the front of vehicles roll past the stop bar as they are waiting for a green signal, the video detectors tend to lose the detection of these vehicles, while the inductive loops do not. Figure 7-3 shows a series of screen captures which illustrate this discrepancy. 


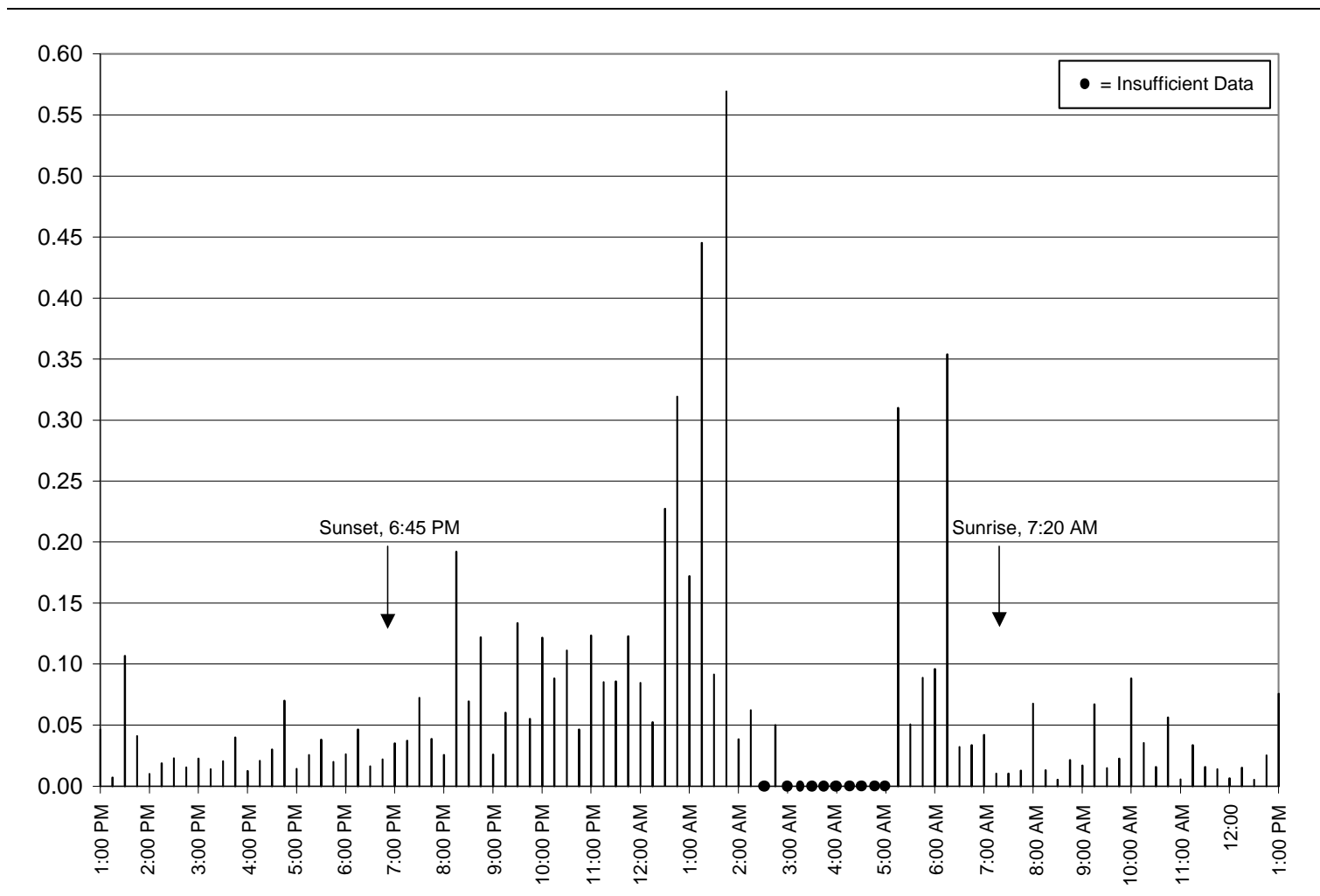

Figure 7-2: System 1 L1V0 Likelihood Profile (Northbound Left Turn Approach, March 4 \& 5, 2001, Overcast) 


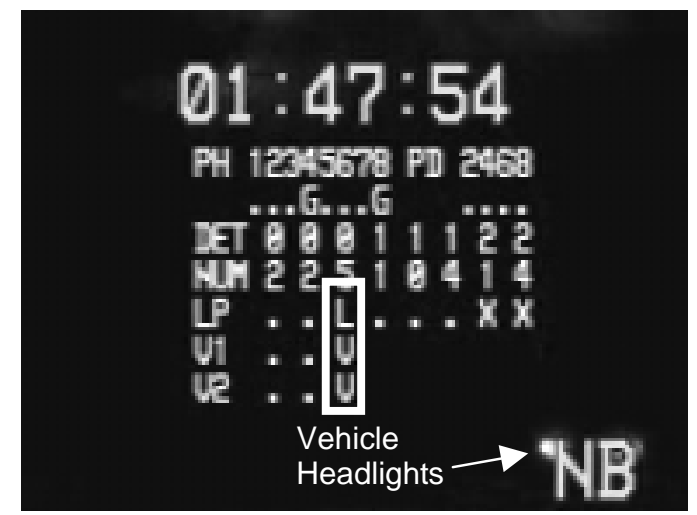

a) Before L1V0 Discrepancy

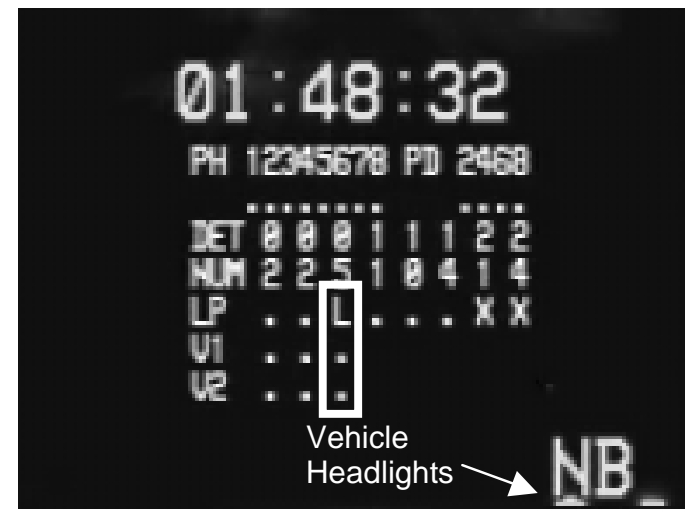

c) During L1V0 Discrepancy Several Seconds Later

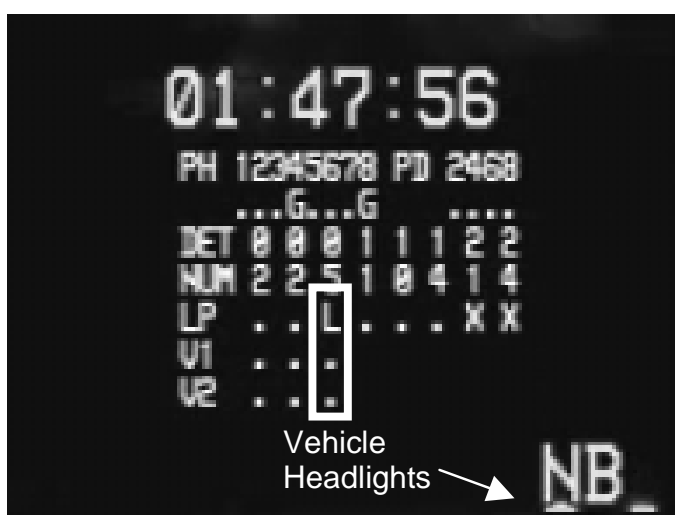

b) During L1V0 Discrepancy

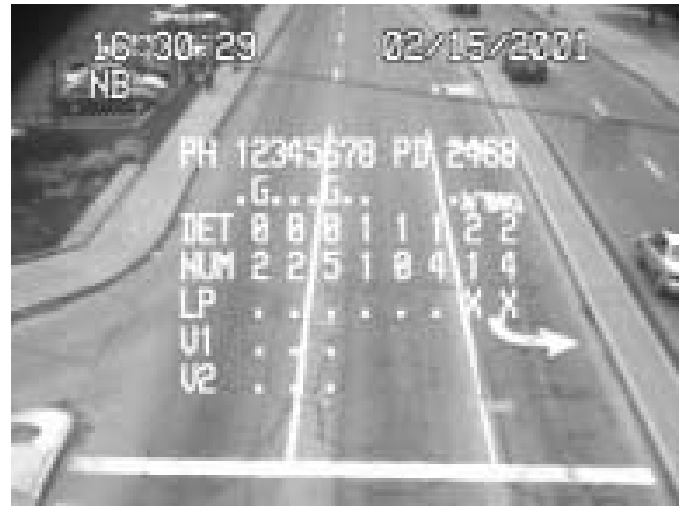

d) Northbound Approach During Daylight to Show Lane Configuration

Figure 7-3: Example Screen Capture of L1V0 Discrepancy Caused by Headlights Being Dropped by Video Detection

\section{Modeling}

In this section, the effects of weather and traffic characteristics on detection discrepancies are modeled. It should be noted that, while inductive loops do introduce their own errors, the marginal effect of most weather characteristics on video detection performance are correctly estimated. This is because most of the weather characteristics do not affect the inductive loops. Therefore, any changes in detection discrepancy due to weather can be entirely associated with the video detectors themselves. 
Because an attempt is made to model likelihoods, two different statistical models were considered: the logit and the probit model. The logit model has been chosen because it is more convenient. The logit model has the following form:

$$
P=\frac{1}{1+e^{-Y}},
$$

where $\mathrm{P}$ is the likelihood of discrepancy, and $\mathrm{Y}$ is the following linear function:

$$
\begin{aligned}
Y= & \beta_{1}+\beta_{2} R+\beta_{3} S N+\beta_{4} \mathrm{WP}+\beta_{5} \mathrm{SU}+\beta_{6} \mathrm{~N}+\beta_{7} \mathrm{MCM} \\
& +\beta_{8} \mathrm{HCM}+\beta_{9} \mathrm{C}+\beta_{10} \mathrm{LT}+\beta_{11} \mathrm{TR},
\end{aligned}
$$

with:

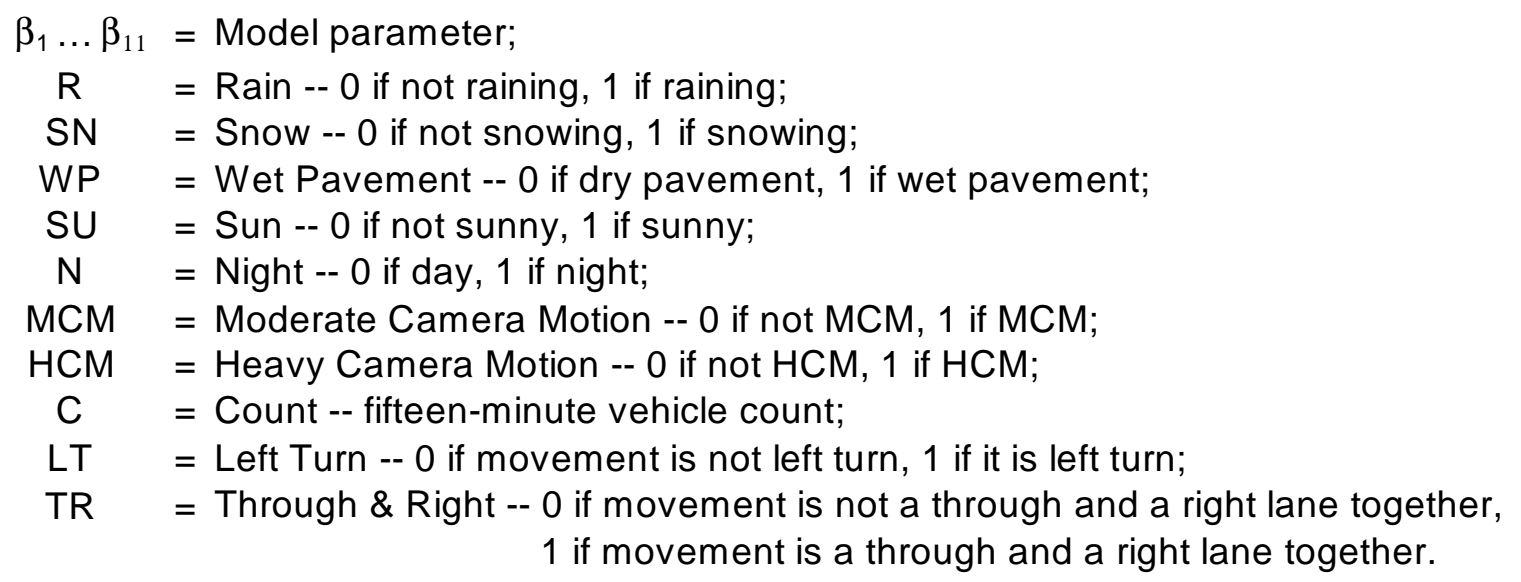

In order to properly calibrate the logit model, the SAS statistical software requires a dependent variable that is a discrete value of either 0 or 1 . In this research, the dependent variable is continuous between 0 and 1 . For this reason, the logit model could not be calibrated with SAS directly. Instead, the model was transformed into the following form:

$$
Y=\ln P-\ln (1-P),
$$


where $\mathrm{Y}$ is a linear function of the independent variables, and $\mathrm{P}=$ the likelihood of discrepancy, as calculated in Equations 4-1 and 4-2. A linear regression was used to calibrate this model.

Because the estimation error of likelihood, $\mathrm{P}$, varies across observations, a weighted linear regression was performed using SAS statistical software. The weight for each observation is the reciprocal of the estimation error of the statistic $\ln P-\ln (1-P)$ in Equation 7-3:

$$
w=\frac{1}{\sqrt{P \cdot(1-P) \cdot D}},
$$

where $\mathrm{P}=$ the likelihood of discrepancy, as calculated in Equations 4-1 and 4-2. $\mathrm{D}=$ the denominator in Equations 4-1 and 4-2.

Four models are needed to estimate the rate of false and missed detections for the two video detection systems. Video detector data for phase 6 was found to be corrupted. All of the observations for phase 6 were dropped from the sample, bringing the number of observations to approximately 560 . All four models were calibrated using the reduced sample. An abbreviated version of the SAS output for the four models is shown in Table 7-3, Table 7-4, Table $7-5$, and Table 7-6. Those rows in the four tables that are in bold type signify the variables that have a statistically significant impact on the performance of video detection at a ten percent level of significance. One of the variables in the tables is 'Base Conditions,' which consists of: no precipitation, overcast skies, daytime, no/low camera motion, very little traffic, and through lanes only. Any deviations from the base conditions are captured through the other variables in the models. These conditions were considered the base conditions because they were assumed to be the optimal conditions for video detection. 
Table 7-3: L1V0 Likelihood Model for System 1

\begin{tabular}{|l|c|c|c|c|}
\hline \multicolumn{1}{|c|}{ Variable } & Parameter Estimate & Standard Error & $\mathrm{t}-$ Value & $\operatorname{Pr}>|\mathrm{t}|$ \\
\hline \hline Base Conditions & $\mathbf{- 2 . 4 6 4 7 2}$ & $\mathbf{0 . 2 1 1 0 3}$ & $\mathbf{- 1 1 . 6 8}$ & $<\mathbf{0 . 0 0 0 1}$ \\
\hline Rain & $\mathbf{0 . 5 8 3 6 2}$ & $\mathbf{0 . 3 2 0 5 7}$ & $\mathbf{1 . 8 2}$ & $\mathbf{0 . 0 6 9 2}$ \\
\hline Snow & $\mathbf{0 . 6 0 6 0 8}$ & $\mathbf{0 . 4 2 5 9 4}$ & $\mathbf{1 . 4 2}$ & $\mathbf{0 . 1 5 5 3}$ \\
\hline Wet Pavement & $\mathbf{- 0 . 4 2 6 0 1}$ & $\mathbf{0 . 3 1 3 7 6}$ & $\mathbf{- 1 . 3 6}$ & $\mathbf{0 . 1 7 5 1}$ \\
\hline Sun & 0.07812 & 0.21422 & 0.36 & 0.7155 \\
\hline Night & $\mathbf{0 . 2 1 2 4 2}$ & $\mathbf{0 . 1 1 7 9 9}$ & $\mathbf{1 . 8 0}$ & $\mathbf{0 . 0 7 2 4}$ \\
\hline Moderate Camera Motion & 0.09827 & 0.14056 & 0.70 & 0.4848 \\
\hline Heavy Camera Motion & 0.09242 & 0.17635 & 0.52 & 0.6004 \\
\hline Average Count & $\mathbf{- 0 . 3 1 2 2 3}$ & $\mathbf{0 . 0 7 2 2 2}$ & $\mathbf{- 4 . 3 2}$ & $<\mathbf{0 . 0 0 0 1}$ \\
\hline Through and Right & $\mathbf{0 . 3 6 5 5 8}$ & $\mathbf{0 . 1 4 7 2 9}$ & $\mathbf{2 . 4 8}$ & $\mathbf{0 . 0 1 3 4}$ \\
\hline Left Turn & $\mathbf{- 0 . 0 5 9 8 7}$ & $\mathbf{0 . 1 4 5 1 3}$ & $\mathbf{- 0 . 4 1}$ & $\mathbf{0 . 6 8 0 1}$ \\
\hline
\end{tabular}

Model $F-$ Value $=5.10$, Model $R^{2}$ Value $=0.085$

Table 7-4: LoV1 Likelihood Model for System 1

\begin{tabular}{|l|c|c|c|c|}
\hline \multicolumn{1}{|c|}{ Variable } & Parameter Estimate & Standard Error & $\mathrm{t}-$ Value & $\operatorname{Pr}>|\mathrm{t}|$ \\
\hline \hline Base Conditions & $\mathbf{- 3 . 6 8 1 6 0}$ & $\mathbf{0 . 1 8 6 6 4}$ & $\mathbf{- 1 9 . 7 3}$ & $<\mathbf{0 . 0 0 0 1}$ \\
\hline Rain & $\mathbf{0 . 7 9 9 4 3}$ & $\mathbf{0 . 2 2 0 3 1}$ & $\mathbf{3 . 6 3}$ & $\mathbf{0 . 0 0 0 3}$ \\
\hline Snow & $\mathbf{0 . 6 9 3 6 9}$ & $\mathbf{0 . 3 6 7 9 1}$ & $\mathbf{1 . 8 9}$ & $\mathbf{0 . 0 5 9 9}$ \\
\hline Wet Pavement & -0.07030 & 0.20997 & -0.33 & 0.7379 \\
\hline Sun & $\mathbf{0 . 7 0 2 9 4}$ & $\mathbf{0 . 2 0 6 1 9}$ & $\mathbf{3 . 4 1}$ & $\mathbf{0 . 0 0 0 7}$ \\
\hline Night & $\mathbf{0 . 8 5 7 8 2}$ & $\mathbf{0 . 1 0 8 2 2}$ & $\mathbf{7 . 9 3}$ & $<\mathbf{0 . 0 0 0 1}$ \\
\hline Moderate Camera Motion & 0.15441 & 0.11736 & 1.32 & 0.1888 \\
\hline Heavy Camera Motion & $\mathbf{0 . 2 8 3 6 2}$ & $\mathbf{0 . 1 5 0 5 3}$ & $\mathbf{1 . 8 8}$ & $\mathbf{0 . 0 6 0 1}$ \\
\hline Average Count & $\mathbf{0 . 3 5 7 3 6}$ & $\mathbf{0 . 0 6 7 4 4}$ & $\mathbf{5 . 3 0}$ & $<\mathbf{0 . 0 0 0 1}$ \\
\hline Through and Right & $\mathbf{1 . 0 4 3 2 3}$ & $\mathbf{0 . 1 3 2 4 4}$ & $\mathbf{7 . 8 8}$ & $<\mathbf{0 . 0 0 0 1}$ \\
\hline Left Turn & $\mathbf{0 . 2 8 8 4 6}$ & $\mathbf{0 . 1 2 4 9 6}$ & $\mathbf{2 . 3 1}$ & $\mathbf{0 . 0 2 1 3}$ \\
\hline
\end{tabular}

Model F-Value $=35.19$, Model $R^{2}$ Value $=0.3906$ 
Table 7-5: L1V0 Likelihood Model for System 2

\begin{tabular}{|l|c|c|c|c|}
\hline \multicolumn{1}{|c|}{ Variable } & Parameter Estimate & Standard Error & $\mathrm{t}-$ Value & $\operatorname{Pr}>|\mathrm{t}|$ \\
\hline \hline Base Conditions & $\mathbf{- 2 . 5 5 4 1 3}$ & $\mathbf{0 . 2 2 4 8 7}$ & $\mathbf{- 1 1 . 3 6}$ & $<\mathbf{0 . 0 0 0 1}$ \\
\hline Rain & 0.40922 & 0.34134 & 1.20 & 0.2311 \\
\hline Snow & $\mathbf{0 . 7 9 4 1 4}$ & $\mathbf{0 . 4 4 0 1 8}$ & $\mathbf{1 . 8 0}$ & $\mathbf{0 . 0 7 1 8}$ \\
\hline Wet Pavement & -0.44757 & 0.32923 & -1.36 & 0.1746 \\
\hline Sun & $\mathbf{0 . 5 6 7 6 9}$ & $\mathbf{0 . 1 9 9 2 9}$ & $\mathbf{2 . 8 5}$ & $\mathbf{0 . 0 0 4 6}$ \\
\hline Night & 0.03550 & 0.12575 & 0.28 & 0.7778 \\
\hline Moderate Camera Motion & 0.03455 & 0.16579 & 0.21 & 0.8350 \\
\hline Heavy Camera Motion & $\mathbf{0 . 3 0 9 7 0}$ & $\mathbf{0 . 1 7 9 2 5}$ & $\mathbf{1 . 7 3}$ & $\mathbf{0 . 0 8 4 6}$ \\
\hline Average Count & $\mathbf{- 0 . 3 3 6 1 2}$ & $\mathbf{0 . 0 7 5 9 3}$ & $\mathbf{- 4 . 4 3}$ & $<\mathbf{0 . 0 0 0 1}$ \\
\hline Through and Right & $\mathbf{0 . 4 1 0 4 2}$ & $\mathbf{0 . 1 7 0 2 0}$ & $\mathbf{2 . 4 1}$ & $\mathbf{0 . 0 1 6 2}$ \\
\hline Left Turn & $\mathbf{0 . 2 6 6 5 2}$ & $\mathbf{0 . 1 5 8 3 2}$ & $\mathbf{1 . 6 8}$ & $\mathbf{0 . 0 9 2 9}$ \\
\hline
\end{tabular}

Model F-Value $=5.66$, Model $R^{2}$ Value $=0.0935$

Table 7-6: LOV1 Likelihood Model for System 2

\begin{tabular}{|l|c|c|c|c|}
\hline \multicolumn{1}{|c|}{ Variable } & Parameter Estimate & Standard Error & $\mathrm{t}-$ Value & $\operatorname{Pr}>|\mathrm{t}|$ \\
\hline \hline Base Conditions & $\mathbf{- 2 . 7 7 1 5 3}$ & $\mathbf{0 . 3 0 7 4 9}$ & $\mathbf{- 9 . 0 1}$ & $<\mathbf{0 . 0 0 0 1}$ \\
\hline Rain & $\mathbf{- 0 . 7 7 0 3 0}$ & $\mathbf{0 . 3 9 4 6 6}$ & $\mathbf{- 1 . 9 5}$ & $\mathbf{0 . 0 5 1 5}$ \\
\hline Snow & -0.76407 & 0.57431 & -1.33 & 0.1839 \\
\hline Wet Pavement & $\mathbf{1 . 6 5 7 5 2}$ & $\mathbf{0 . 3 8 4 8 9}$ & $\mathbf{4 . 3 1}$ & $<\mathbf{0 . 0 0 0 1}$ \\
\hline Sun & -0.06667 & 0.38953 & -0.17 & 0.8642 \\
\hline Night & 0.01512 & 0.18168 & 0.08 & 0.9337 \\
\hline Moderate Camera Motion & 0.14544 & 0.19755 & 0.74 & 0.4619 \\
\hline Heavy Camera Motion & $\mathbf{- 1 . 2 4 7 0 3}$ & $\mathbf{0 . 2 8 2 5 4}$ & $\mathbf{- 4 . 4 1}$ & $<\mathbf{0 . 0 0 0 1}$ \\
\hline Average Count & $\mathbf{0 . 2 8 5 6 8}$ & $\mathbf{0 . 1 1 5 2 2 7}$ & $\mathbf{2 . 4 8}$ & $\mathbf{0 . 0 1 3 4}$ \\
\hline Through and Right & $\mathbf{0 . 7 3 8 8 2}$ & $\mathbf{0 . 2 1 8 4 4}$ & $\mathbf{3 . 3 8}$ & $\mathbf{0 . 0 0 0 8}$ \\
\hline Left Turn & $\mathbf{0 . 4 5 2 5 9}$ & $\mathbf{0 . 2 0 5 2 1}$ & $\mathbf{2 . 2 1}$ & $\mathbf{0 . 0 2 7 8}$ \\
\hline
\end{tabular}

Model F-Value $=10.4$, Model $R^{2}$ Value $=0.1593$

The resulting models have low $R^{2}$ values, which means that there are factors of video detection performance that have not been included in these models. Most likely, the differences between the two detection techniques cause the unexplained discrepancy. For example, the occupancy times in video detection are affected by the height of vehicles, while the occupancy times of inductive loops are not. Capturing the effect of vehicle height would require vehicle classification, which is beyond the scope of this research. 


\section{Discussion of Results}

As mentioned previously, the results of the four models of detection discrepancies can be used directly to determine the effect of the studied weather conditions on video detection. Using the model calibrated in the previous section, Table 7-7 through Table 7-10 give an estimation of the effects of various weather characteristics. Only those variables that are statistically significant are shown in these tables.

Table 7-7: Sensitivity of the L1V0 Likelihood Model for System 1

\begin{tabular}{|l|c|c|}
\hline \multicolumn{1}{|c|}{ Factor } & Parameter Value & Change from base conditions \\
\hline \hline Base Conditions ${ }^{(1)}$ & -2.4647 & $0.00 \%^{(2)}$ \\
\hline Rain & 0.5836 & $5.39 \%$ \\
\hline Night & 0.2124 & $1.68 \%$ \\
\hline Average Count & -0.3122 & $-1.98 \%$ \\
\hline Through \& Right & 0.3656 & $3.08 \%$ \\
\hline
\end{tabular}

${ }^{1}$ Base Conditions: dry pavement, no precipitation, overcast, no/low camera motion, very low volume, through movement

2) Likelihood of L1V0 discrepancy for the base condition is $7.84 \%$

Table 7-8: Sensitivity of the LOV1 Likelihood Model for System 1

\begin{tabular}{|l|c|c|}
\hline \multicolumn{1}{|c|}{ Factor } & Parameter Value & Change from Base Conditions \\
\hline \hline Base Conditions ${ }^{(1)}$ & -3.6816 & $0.00 \%{ }^{(2)}$ \\
\hline Rain & 0.7994 & $2.85 \%$ \\
\hline Snow & 0.6937 & $2.34 \%$ \\
\hline Sun & 0.7029 & $2.38 \%$ \\
\hline Night & 0.8578 & $3.15 \%$ \\
\hline Hvy. Cam. Motion & 0.2836 & $0.78 \%$ \\
\hline Average Count & 0.3574 & $1.02 \%$ \\
\hline Left Turn & 0.2885 & $0.79 \%$ \\
\hline Through \& Right & 1.0432 & $4.21 \%$ \\
\hline
\end{tabular}

1) Base Conditions: dry pavement, no precipitation, overcast, no/low camera motion, very low volume, through movement

2) Likelihood of LOV1 discrepancy for the base condition is $2.46 \%$ 
Table 7-9: Sensitivity of the L1V0 Likelihood Model for System 2

\begin{tabular}{|l|c|c|}
\hline \multicolumn{1}{|c|}{ Factor } & Parameter Value & Change from base conditions \\
\hline \hline Base Conditions ${ }^{(1)}$ & -2.55413 & $0.00 \%{ }^{(2)}$ \\
\hline Snow & 0.79414 & $7.46 \%$ \\
\hline Sun & 0.56769 & $4.85 \%$ \\
\hline Hvy. Cam. Motion & 0.3097 & $2.37 \%$ \\
\hline Average Count & -0.33453 & $-1.94 \%$ \\
\hline Left Turn & 0.26652 & $2.00 \%$ \\
\hline Through \& Right & 0.41042 & $3.28 \%$ \\
\hline
\end{tabular}

${ }^{1)}$ Base Conditions: dry pavement, no precipitation, overcast, no/low camera motion, very low volume, through movement

${ }^{2)}$ Likelihood of L1V0 discrepancy for the base condition is $7.21 \%$

Table 7-10: Sensitivity of the LOV1 Likelihood Model for System 2

\begin{tabular}{|l|c|c|}
\hline \multicolumn{1}{|c|}{ Factor } & Parameter Value & Change from base conditions \\
\hline \hline Base Conditions $^{(1)}$ & -2.77153 & $0.00 \%{ }^{(2)}$ \\
\hline Rain & -0.7703 & $-3.07 \%$ \\
\hline Wet Pavement & 1.65752 & $18.82 \%$ \\
\hline Hvy. Cam. Motion & -1.24703 & $-4.12 \%$ \\
\hline Average Count & 0.285678 & $1.80 \%$ \\
\hline Left Turn & 0.73882 & $5.69 \%$ \\
\hline Through \& Right & 0.45259 & $3.07 \%$ \\
\hline
\end{tabular}

${ }^{1)}$ Base Conditions: dry pavement, no precipitation, overcast, no/low camera motion, very low volume, through movement

${ }^{2)}$ Likelihood of LOV1 discrepancy for the base condition is $5.89 \%$

It appears that under the base conditions, System 2 is over twice more likely (5.9\%) to have false detection than System 1 (2.5\%) (Table 7-8 and Table 7-10). Both systems are estimated to have a missed vehicle presence between seven and eight percent of the time under the base conditions (Table 7-7 and Table 7-9). Therefore, System 1 appears to perform better under the base conditions.

Table 7-7 through Table 7-10 present a sensitivity analysis of the developed models. The last column shows the change in video detection performance from the base conditions caused by particular factors. For example, 
Table 7-8, shows that night conditions are associated with a higher instance of false detection for System 1 than in the base conditions.

There are several characteristics that degrade the performance of video detection by more than five percent. According to the System 1 L1V0 Discrepancy model (Table 7-7), rain causes 5.39\% more missed or dropped detections than base conditions. System 1 tends to generate false detection under diverse conditions infrequently, as there are no factors that degrade the system performance by more than $5 \%$ (Table $7-8$ ).

According to the System 2 L1V0 Discrepancy model, snow causes more missed detections than the base conditions since the likelihood increases be $7.46 \%$ (Table 7-9). It should be noted that there is a limited amount of snow data in the sample, so caution should be used when making any generalizations of this finding. Sun, or shadowy conditions, also caused a $4.85 \%$ increase in the likelihood of missed detection by System 2. At first thought, this result seems to go against intuition; however the sun causes a great deal of glare, which at times can even 'blind' the cameras. According to Table 7-10, wet pavement causes $18.8 \%$ more LOV1 discrepancy than in the base conditions. This is due to the glare of headlights and sun off the wet pavement. Therefore, according to this fourth model, it can safely be suggested that when using System 2 during times when the pavement is wet, the capacity of the intersection could be reduced. Also, System 2 seemed to have difficulties with the left turn movements, which experience $5.7 \%$ greater false detection than the through movement. This is because there were several times when System 2 continually indicated the presence of a vehicle, or went into recall, in the left turn lanes. We cannot give an explanation for this behavior. It is also difficult to explain why the System 2 performance improvement is associated with rain and heavy camera motion. 
An investigation of the best- and worse-case scenarios may provide some added insight into the video detection evaluation. The performance of both of the video detection systems under the base conditions has already been discussed, and therefore it would be appropriate to discuss a "worst-case" scenario, as well. The following weather conditions constitute the worst-case scenario: rain, night, wet pavement, average count, heavy camera motion, and signal phase eight, which has through and right turn detectors combined together. Under these conditions, System 1 misses over $16 \%$ of presence time, and $40 \%$ of vehicle absence time is incorrectly indicated as presence (false detection).

Under poor conditions, System 2 misses $20 \%$ of vehicle presence time. Because rain does not affect the ability of System 2 in detecting vehicle presence, snow was instead used as one of the factors in determining how often System 2 missed vehicle presence. Without considering the effects of rain and heavy camera motion, System 2 also places false detection for over $40 \%$ of the vehicle absence time.

In reality, the poorest conditions will take place very infrequently. Under the base conditions, both systems miss vehicle presence between $7 \%$ and $8 \%$ of vehicle presence time (Table 7-7 and Table 7-9). Also under base conditions, System 1 and System 2 generate false detections approximately $2.5 \%$ and $6 \%$, respectively (Table 7-8 and Table 7-10).

\section{Errors of Loop Detection}

The purpose of this section is to model the performance of inductive loop detectors with respect to volume and pavement conditions. Once the model is calibrated and the error rates of inductive loops under various conditions can be estimated, the results of this model may be combined with the results of the previous section to estimate the absolute error rates of video detection. 
The purpose of this section of the report is to show the procedure that can be used to perform a more in-depth study, and not to perform a comprehensive ground truth evaluation for all of the loops at the instrumented intersection.

\section{Data Collection}

In order to determine the error rates for the inductive loops used in this research, it was necessary to use a limited amount of human observation. Because the image with all of the approaches and the text overlay was not sufficiently clear to see the inductive loops, it was necessary to use the pan-tiltzoom (PTZ) camera (Figure 3-4).

In the case that a PTZ camera is not available, a normal video camcorder may be used to collect the necessary footage. In fact, at one point during this research, the PTZ camera was malfunctioning, and thus there was a need to use a camcorder. If it is necessary to use a camcorder, careful attention must be given to the proper placement of the camera to have a good view of the inductive loop detectors being evaluated. In addition, only high-quality media should be used for the data collection in order to minimize the amount of error.

The PTZ camera has the capability of zooming in on specific inductive loops. For most of the data collections, the camera was zoomed in on one specific set of downstream loops (ie., those that control phase 5, shown in Figure 7-4). After the video was collected of both wet and dry weather, an observer watched the video in order to extract the ground truth data. To do this, the inductive loops were marked on the computer screen using pieces of tape. A Hewlett Packard 48GX handheld calculator was programmed to perform as a manual push-button detector. One button was selected to record the detection start time, and another to record the detection end time. Time was measured 
from the onset of data collection. Whenever a vehicle entered the empty detection zone, the start button was pushed on the calculator. When the last vehicle in the detection zone left, the stop button was pushed. The observer also counted the number of vehicles entering the detection zone. Data were aggregated in fifteen-minute intervals. At the end of each data collection (two hour segments), the calculator was hooked to a PC, and the data was uploaded into a spreadsheet program.

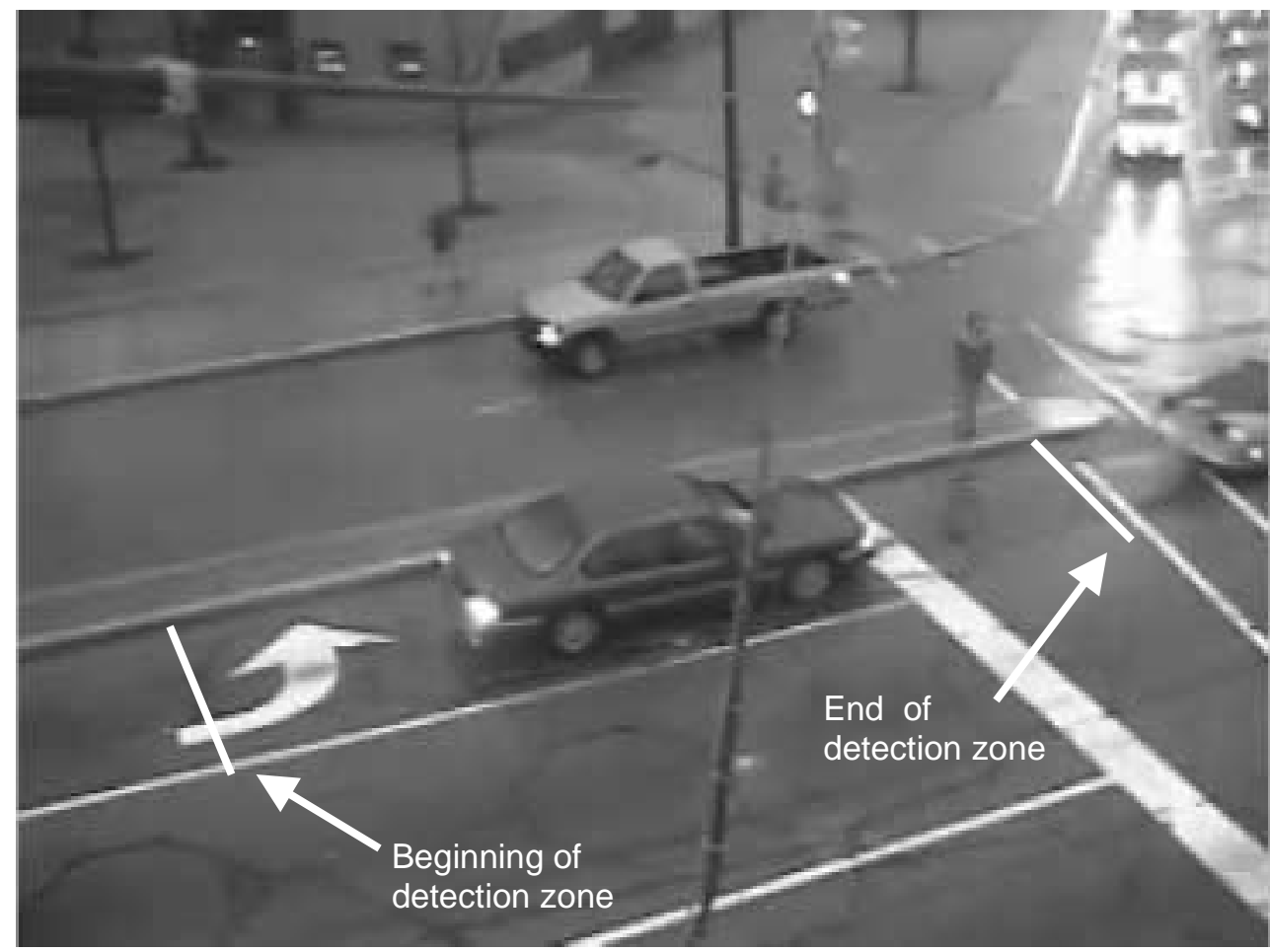

Figure 7-4: Screen Capture of Northbound Left Turn Lane During a Ground Truth Data Collection

Inside the spreadsheet program, the start times were subtracted from the stop times to give the occupancy times of the inductive loop. Using these occupancy times, the data was compared to the data collected using the Opto Controller (Figure 3-14). Several occupancy times were matched, in which the 
author was sure that they were the same detection event, and after about thirty observations were matched, the start and stop times were compared.

After a preliminary run of two hours of data, it was determined that the data collected from the inductive loops and the ground truth data were on different time scales, and that the relationship between the time scales was linear. Using a linear regression of the form shown in Equation 7-5 for approximately thirty observations, the ground truth time scale was adjusted to match that of the Opto Controller time scale,

$$
L=\beta_{1}+T \beta_{2} .
$$

In Equation 7-5, $L=$ inductive loop time and $T=$ ground truth time. The $\beta_{1}$ parameter gives the shift in the time scales, and $\beta_{2}$ gives the adjustment factor. Table 7-11 gives the time shift and adjustment factor for each of the data collection periods. The adjustment factors were consistently 1.01 , meaning that the time scales of the Opto Controller and the HP Calculator differed by approximately one percent throughout all of the ground truth data collection sessions. The time shift and adjustment factors of Table 7-11 allow for direct comparison of the ground truth data and the inductive loop data. This procedure was followed for the rest of the ground truth data collection, as well. 
Table 7-11: Shift and Adjustment Factor for Ground Truth Time

\begin{tabular}{|c|r|r|}
\hline Data Collection \# & Time Shift $\left(\beta_{1}, \mathrm{sec}\right)$ & Adjustment Factor $\left(\beta_{2}\right)$ \\
\hline \hline 1 & 64363.27 & 1.0139 \\
\hline 2 & 71569.33 & 1.0107 \\
\hline 3 & 78881.03 & 1.0107 \\
\hline 4 & 86101.42 & 1.0107 \\
\hline 5 & 93364.19 & 1.0104 \\
\hline 6 & 100566.08 & 1.0108 \\
\hline 7 & 107791.99 & 1.0108 \\
\hline 8 & 115265.79 & 1.0107 \\
\hline 9 & 65043.02 & 1.0111 \\
\hline 10 & 57318.80 & 1.0101 \\
\hline
\end{tabular}

\begin{abstract}
Modeling
To model the performance of the inductive loops, it is first necessary to determine the weather and traffic characteristics that could possibly have an affect on inductive loop detectors. There are not nearly as many factors that affect inductive loops as do video detectors. The only characteristic related to weather that could affect inductive loop performance is wet pavement. Traffic volume, different vehicle types, and different installation procedures could also have an effect on detection quality. A model is to be developed to capture the effects of wet pavement and vehicle intensity on inductive loop performance, as well as the difference between the detection quality of different loops.
\end{abstract}

The logit model as in Equation 7-1 was used to describe the likelihood of inductive loop errors, where $Y$ is the following linear function:

$$
Y=\beta_{1}+\beta_{2} W P+\beta_{3} C+\beta_{4} P h 7
$$

with :

$\beta_{1} \ldots \beta_{4}=$ model parameters;

$W P=$ Wet Pavement -0 if pavement is dry, and 1 if it is wet; 
$\mathrm{C} \quad=15$-minute vehicle Count;

$\mathrm{Ph} 7=$ Phase $7-0$ if data is for Phase 5, 1 if it is for Phase 7 .

Equations 7-3 and 7-4 were again used for weighted linear regression. Two models were calibrated - one for TOL1 errors, and one for T1L0 errors. An abbreviated version of the SAS output for the two models is shown in Table 7-12 and Table 7-13. The rows in these tables that are bolded include those variables that have a significant impact upon the operation of inductive loop detectors.

Table 7-12: TOL1 Likelihood Model for Loop Ground Truth

\begin{tabular}{|l|c|c|c|c|}
\hline \multicolumn{1}{|c|}{ Variable } & Parameter Estimate & Standard Error & $\mathrm{t}-$ Value & $\operatorname{Pr}>|\mathrm{t}|$ \\
\hline \hline Base Conditions & $\mathbf{- 5 . 7 6 8 2 3}$ & $\mathbf{0 . 2 2 5 3 9}$ & $\mathbf{- 2 5 . 5 9}$ & $<\mathbf{0 . 0 0 0 1}$ \\
\hline Wet Pavement & -0.20720 & 0.20460 & -1.01 & 0.3145 \\
\hline Average Count & $\mathbf{1 . 7 0 5 5 8 7}$ & $\mathbf{0 . 1 3 9 8 5 9}$ & $\mathbf{1 2 . 2}$ & $<\mathbf{0 . 0 0 0 1}$ \\
\hline Phase 7 & $\mathbf{2 . 4 7 7 8 5}$ & $\mathbf{0 . 3 6 3 8 4}$ & $\mathbf{6 . 8 1}$ & $<\mathbf{0 . 0 0 0 1}$ \\
\hline
\end{tabular}

Model F-Value $=67.61$, Model R2 Value $=0.7327$

Table 7-13: T1L0 Likelihood Model for Loop Ground Truth

\begin{tabular}{|l|c|c|c|c|}
\hline \multicolumn{1}{|c|}{ Variable } & Parameter Estimate & Standard Error & $\mathrm{t}-$ Value & $\operatorname{Pr}>|\mathrm{t}|$ \\
\hline \hline Base Conditions & $\mathbf{- 6 . 3 2 9 7 5}$ & $\mathbf{0 . 7 5 5 9 7}$ & $\mathbf{- 8 . 3 7}$ & $<\mathbf{0 . 0 0 0 1}$ \\
\hline Wet Pavement & $\mathbf{- 1 . 6 1 2 4 3}$ & $\mathbf{0 . 7 1 5 3 2}$ & $\mathbf{- 2 . 2 5}$ & $\mathbf{0 . 0 2 7 1}$ \\
\hline Average Count & 0.151117 & 0.368916 & 0.41 & 0.6830 \\
\hline Phase 7 & $\mathbf{3 . 1 8 9 4 7}$ & $\mathbf{1 . 7 0 1 9 3}$ & $\mathbf{1 . 8 7}$ & $\mathbf{0 . 0 6 4 9}$ \\
\hline
\end{tabular}

Model F-Value $=4.78$, Model $R^{2}$ Value $=0.1625$

Discussion of Results

Results of a sensitivity analysis of the two models calibrated in the previous section are given in Table 7-14 and Table 7-15. Only those factors that have a $10 \%$ level of significance are shown in these tables. The base conditions for this model are the Phase 5 loops, very low/no traffic, and dry pavement. 
Table 7-14: Sensitivity of the TOL1 Likelihood Model for Loop Ground Truth

\begin{tabular}{|l|c|c|}
\hline \multicolumn{1}{|c|}{ Factor } & Parameter Estimate & Change from Base Conditions \\
\hline \hline Base Conditions ${ }^{(1)}$ & -5.76823 & $0.0000 \%{ }^{(2)}$ \\
\hline Average Count & 1.70559 & $1.3797 \%$ \\
\hline Phase 7 & 2.47785 & $3.2787 \%$ \\
\hline
\end{tabular}

${ }^{1)}$ Base Conditions: dry pavement, very low volume, Phase 5 loops

2) Likelihood of TOL1 discrepancy for the base condition is $0.31 \%$

Table 7-15: Sensitivity of the T1L0 Likelihood Model for Loop Ground Truth

\begin{tabular}{|l|c|c|}
\hline \multicolumn{1}{|c|}{ Factor } & Parameter Estimate & Change from Base Conditions \\
\hline \hline Base Conditions ${ }^{(1)}$ & -6.32975 & $0.0000 \%^{(2)}$ \\
\hline Wet Pavement & -1.61243 & $-0.1157 \%$ \\
\hline Phase 7 & 3.18947 & $6.8739 \%$ \\
\hline
\end{tabular}

${ }^{1)}$ Base Conditions: dry pavement, very low volume, Phase 5 loops

2) Likelihood of T1L0 discrepancy for the base condition is $0.18 \%$

There is a general finding prompted by these two models. The inductive loops perform very well in the base conditions. The wet pavement and traffic volume impacts, although statistically significant, do not deteriorate the loop operation to an unacceptable extent.

The difference between the performance of the inductive loop considered for base conditions (Phase 5) and the other loop included in the model (Phase 7) is relatively large. This means that different inductive loops perform differently, and should be ground truthed separately if an in-depth investigation is to be performed. In other words, if one wants to incorporate the inductive loop error into the evaluation of all approaches at an intersection, it is necessary to ground truth the inductive loops at each approach. These models were re-calibrated without the Phase 7 variable. The $\mathrm{R}^{2}$ value and the model parameters changed very slightly, therefore the original form of the two models was kept. 
One can conclude from Table 7-14 that wet pavement does not affect the likelihood of TOL1 error. Traffic volume; however, does affect the likelihood of TOL1 error. If there are 43.3 vehicles in fifteen minutes, the likelihood of TOL1 error increases by $1.38 \%$. There is a possible explanation why traffic volume may affect the likelihood of TOL1 error. If the volume increases in the left turn lane, it is also increasing in the adjacent through lanes. With this increase in volume in the adjacent lane comes an increase in the possibility of detecting vehicles from the adjacent lane or cross-talk between the loop detectors.

Table 7-15 shows that traffic volume does not have a significant impact upon the likelihood of T1L0 error, or missed detection, while wet pavement reduces the likelihood of T1L0 error.

If the likelihood of loop error is found to be high, it should be incorporated into the video-loop discrepancy model to determine the likelihood of video detection error. If the likelihood of loop error is extremely low, then it may be excluded from the evaluation, and the video-loop discrepancy model may be used to determine the likelihood of video detection error.

\section{Errors of Video Detection}

There are two different methods of finding the likelihood of video detection errors. The first method is to compare the output of video detection to human observation. Because the video detectors can be easily repositioned, human observations must be repeated every time the detectors are changed. This method is labor intensive. The second method uses a limited amount of human observation to find the likelihood of inductive loop errors. Then, the likelihoods of inductive loop errors can be combined with the likelihoods of discrepancy between video detection and inductive loop detection to incorporate the effect of 
loop imperfection. The detection discrepancies can be estimated using an automated technique such as the one described earlier in this report.

\section{Modeling}

The definitions of likelihoods of video-loop discrepancy and of inductive loop error can be used directly to calculate the likelihood of video detection error. Figure 7-5 shows a tree of events that is useful in deriving the conditional likelihood equations for video detection error:

$$
\begin{aligned}
\mathrm{P}\left(\mathrm{V}=\left.0\right|_{\mathrm{T}=1}\right) & =\mathrm{P}\left(\mathrm{L}=\left.1\right|_{\mathrm{T}=1}\right) \cdot \mathrm{P}\left(\mathrm{V}=\left.0\right|_{\mathrm{L}=1}\right) \\
& +\mathrm{P}\left(\mathrm{L}=\left.0\right|_{\mathrm{T}=1}\right) \cdot \mathrm{P}\left(\mathrm{V}=\left.0\right|_{\mathrm{L}=0}\right) \\
\mathrm{P}\left(\mathrm{V}=\left.1\right|_{\mathrm{T}=0}\right) & =\mathrm{P}\left(\mathrm{L}=\left.1\right|_{\mathrm{T}=0}\right) \cdot \mathrm{P}\left(\mathrm{V}=\left.1\right|_{\mathrm{L}=1}\right) \\
& +\mathrm{P}\left(\mathrm{L}=\left.0\right|_{\mathrm{T}=0}\right) \cdot \mathrm{P}\left(\mathrm{V}=\left.1\right|_{\mathrm{L}=0}\right)
\end{aligned}
$$

where $\mathrm{P}$ indicates a likelihood value, $\mathrm{L}=$ inductive loop detector, $\mathrm{V}=$ video detector, $T=$ ground truth, $0=$ does not indicate presence, and $1=$ indicates presence. The likelihood values in Equations 7-7 and 7-8 incorporate the imperfection of inductive loops. 


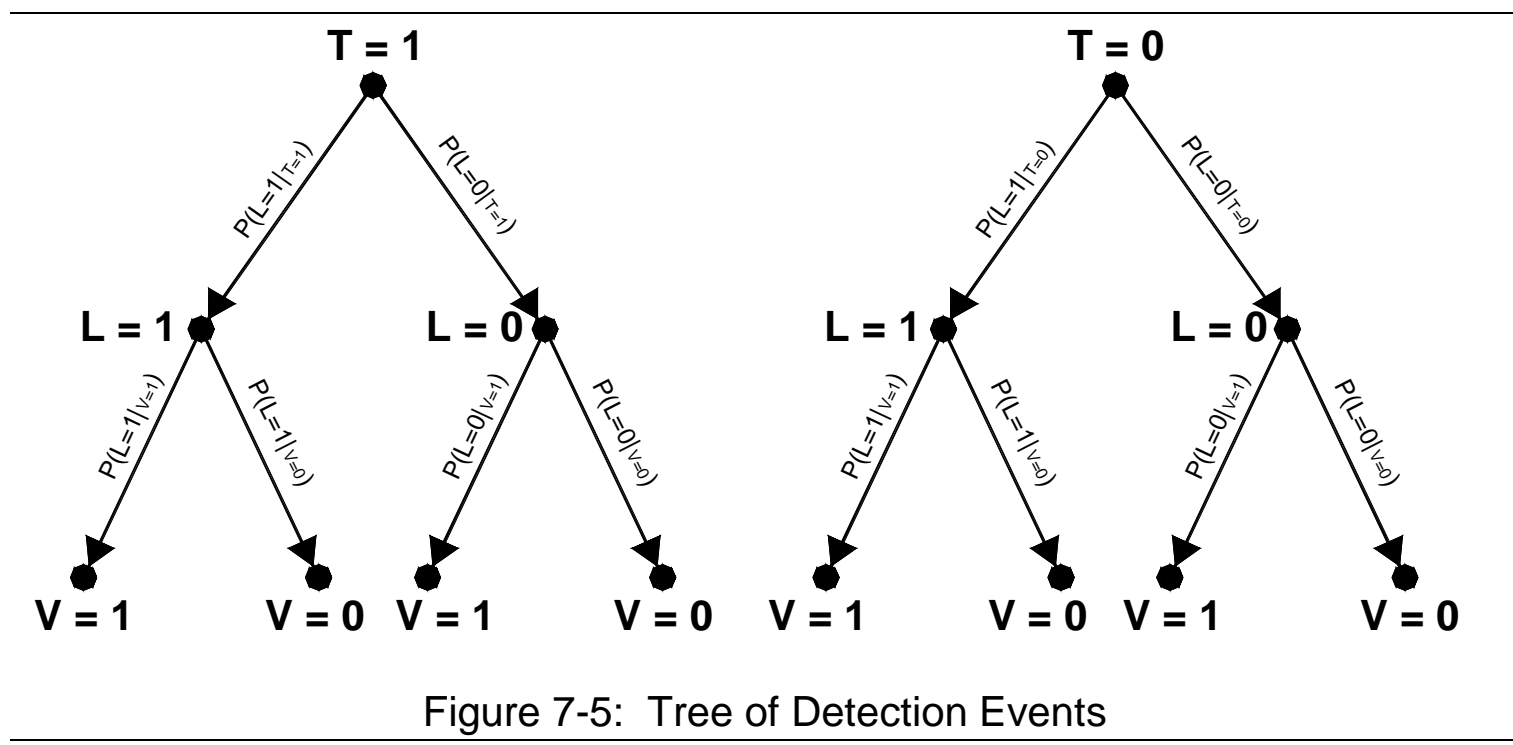

\section{Example Profiles of Video Detection Error Likelihoods}

Using Equations 7-7 and 7-8, the likelihood of video detection error was estimated for the Phase 5 inductive loops over a period of one day. Figure 7-6 shows both the L1V0 discrepancy profile and the T1V0 error profile for comparison. It appears that there is very little difference between the two profiles, indicating that loop imperfection is too small to have a considerable effect on the missed detections for Phase 5. 


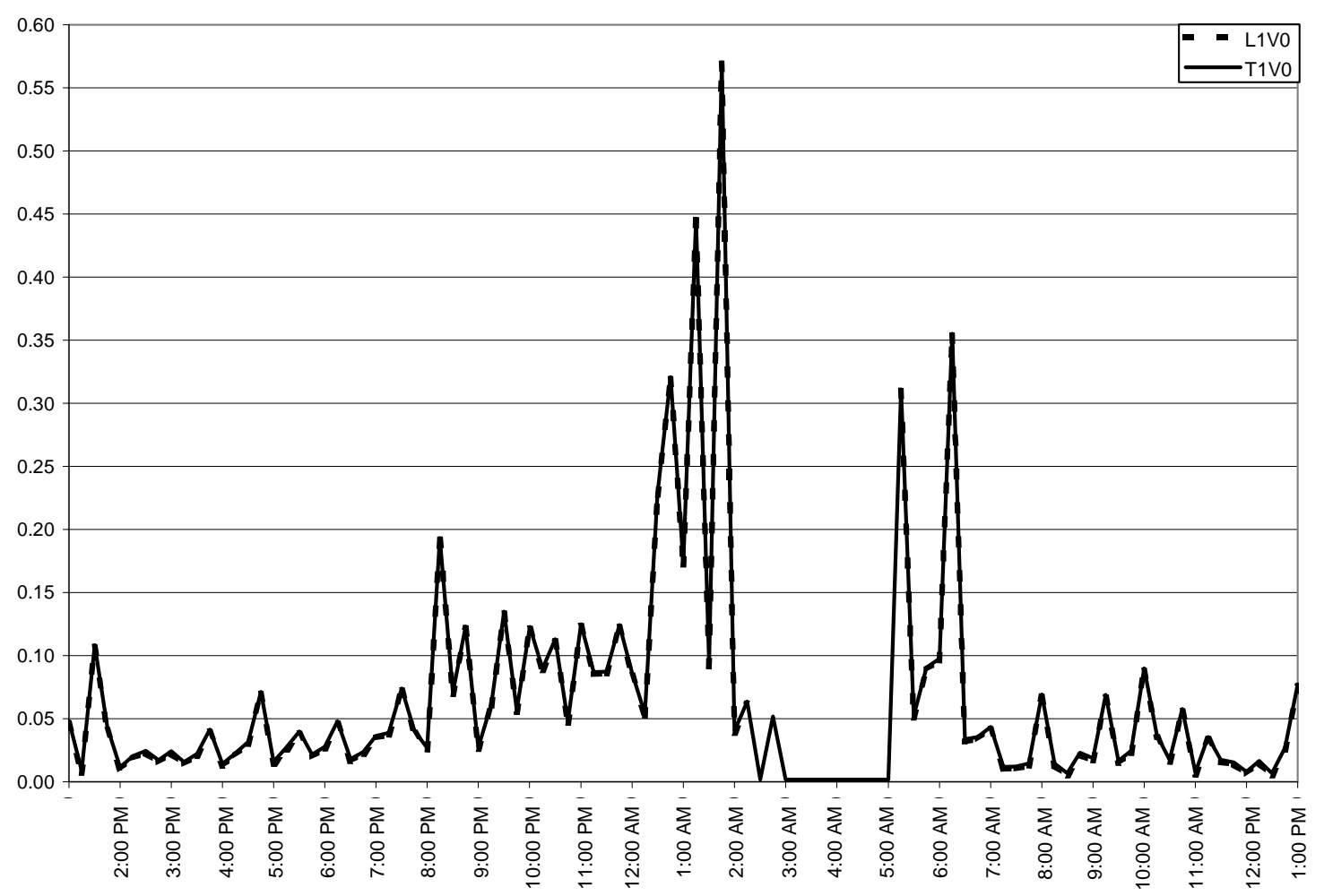

Figure 7-6: System 1 L1V0 vs. T1V0 Likelihood Profile (Northbound Left Turn Approach, March 4 \& 5, 2001, Overcast)

Figure 7-7 shows both the LOV1 discrepancy profile and the TOV1 error profile over the same day. This figure shows that the imperfection of the loops does have an effect on the estimated false detection likelihood. This figure implies that during the night, when the traffic volume is low, the imperfection of the loops is not as great of an issue as it is during the high volume times of the day. According to the profiles, as traffic volume increases, incorporating loop imperfection into the model causes the estimation of false detections to rise. 


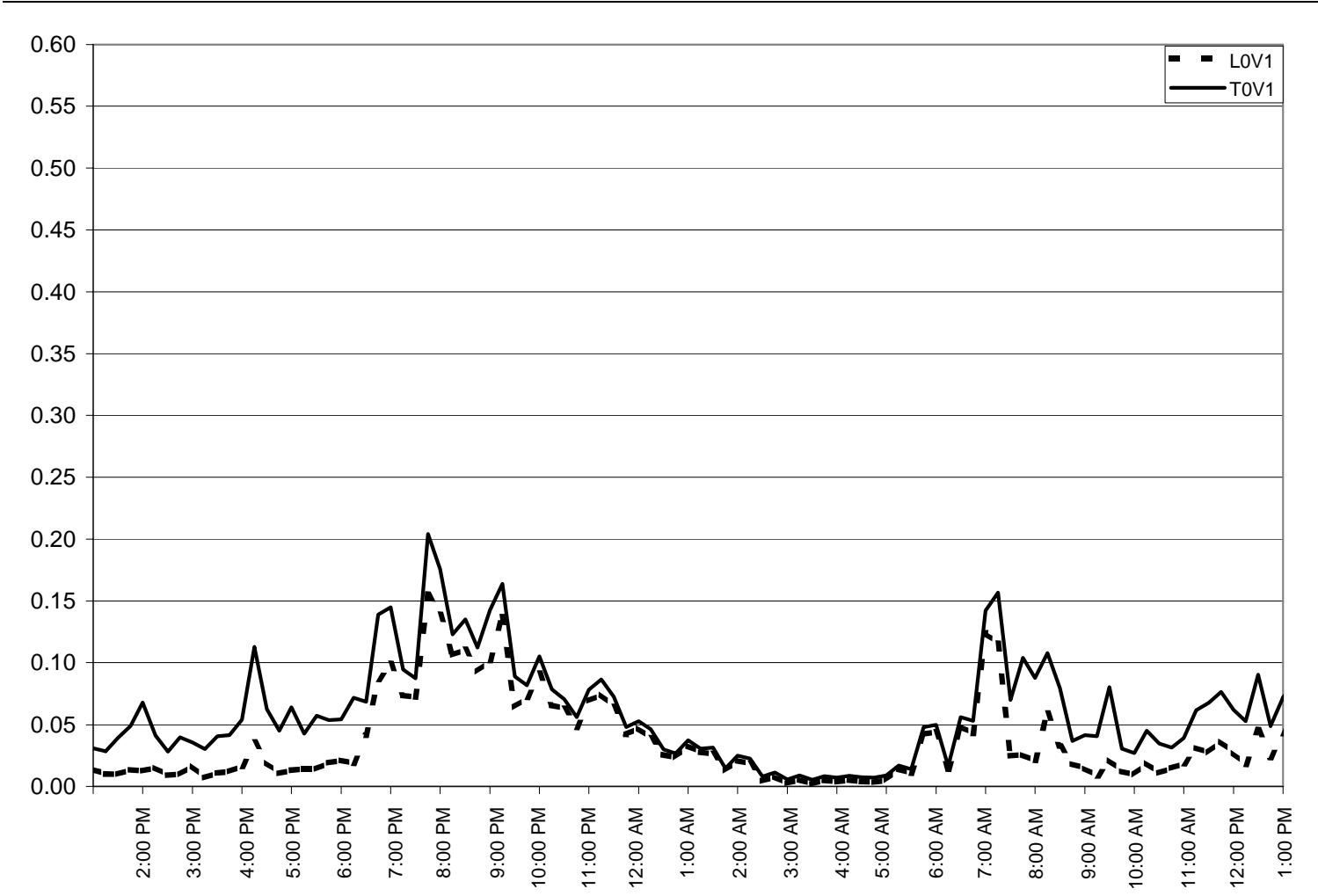

Figure 7-7: System 1 LOV1 vs. TOV1 Likelihood Profile (Northbound Left Turn Approach, March 4 \& 5, 2001, Overcast)

One of the most important points that can be made using Figure 7-6 and Figure 7-7 is that because the inductive loops have an extremely low percentage of missed vehicle presence, the loop-video discrepancy model for estimating missed vehicle presence gives nearly the exact same output as the video detector error model for estimating missed vehicle presence (Figure 7-6). Because inductive loops sometimes falsely generate vehicle presence as the volume increases, the loop-video discrepancy model should not be used during times of high volumes in order to determine the amount of false detection generated by video detectors (Figure 7-7). This is because the inductive loops cause the loop-video discrepancy model to predict with less accuracy during these times. If the loop error rates are taken into consideration, and the video error model is used, then the predictions of this model are more realistic during times of high volume. 
Consider these example calculations of the values used for T1V0 and T0V1 in Figure 7-6 and Figure 7-7:

$$
\text { At 5:00 PM, } \begin{aligned}
& \mathrm{P}\left(\mathrm{V}=\left.0\right|_{\mathrm{L}=1}\right)=0.0142 \\
& \mathrm{P}\left(\mathrm{V}=\left.1\right|_{\mathrm{L}=0}\right)=0.0130 \\
& \mathrm{P}\left(\mathrm{V}=\left.0\right|_{\mathrm{L}=0}\right)=1-\mathrm{P}\left(\mathrm{V}=\left.1\right|_{\mathrm{L}=0}\right)=1-0.0130=0.9870 \\
& \mathrm{P}\left(\mathrm{V}=\left.1\right|_{\mathrm{L}=1}\right)=1-\mathrm{P}\left(\mathrm{V}=\left.0\right|_{\mathrm{L}=1}\right)=1-0.0142=0.9858 \\
& \mathrm{P}\left(\mathrm{L}=\left.0\right|_{\mathrm{T}=1}\right)=0.0018 \\
& \mathrm{P}\left(\mathrm{L}=\left.1\right|_{\mathrm{T}=0}\right)=0.0532 \\
& \mathrm{P}\left(\mathrm{L}=\left.0\right|_{\mathrm{T}=0}\right)=1-\mathrm{P}\left(\mathrm{L}=\left.1\right|_{\mathrm{T}=0}\right)=1-0.0532=0.9468 \\
& \mathrm{P}\left(\mathrm{L}=\left.1\right|_{\mathrm{T}=1}\right)=1-\mathrm{P}\left(\mathrm{L}=\left.0\right|_{\mathrm{T}=1}\right)=1-0.0018=0.9982 .
\end{aligned}
$$

According to the above calculations and Equation 7-7,

$$
\mathrm{P}\left(\mathrm{V}=\left.0\right|_{\mathrm{T}=1}\right)=0.9982 \cdot 0.0142+0.0018 \cdot 0.9870=0.0160 \text {. }
$$

Likewise, according to Equation 7-8,

$$
P\left(V=\left.1\right|_{T=0}\right)=0.0532 \cdot 0.9858+0.9468 \cdot 0.0130=0.0648 .
$$

One may notice that the value of 0.0160 for $P\left(V=\left.0\right|_{T=1}\right)$ is very close to that of 0.0142 for $P\left(V=\left.0\right|_{L=1}\right)$, as can be seen in Figure 7-6 at 5:00 PM. On the other hand, the value of 0.0648 calculated for $P\left(V=\left.1\right|_{T=0}\right)$ is much greater than 0.0130 for, as can be seen in Figure 7-7, also at 5:00 PM. The reason for this large difference is due to the high volume at 5:00 PM.

If one would consider a low-volume time of the night, such as 2:00 AM, the values would be much closer between $\mathrm{P}\left(\mathrm{V}=\left.1\right|_{\mathrm{T}=0}\right)$ and $\mathrm{P}\left(\mathrm{V}=\left.1\right|_{\mathrm{L}=0}\right)$, as is shown by the following example calculation:

$$
\text { At 2:00 AM, } \begin{aligned}
& \mathrm{P}\left(\mathrm{V}=\left.0\right|_{\mathrm{L}=1}\right)=0.0385 \\
& \mathrm{P}\left(\mathrm{V}=\left.1\right|_{\mathrm{L}=0}\right)=0.0209 \\
& \mathrm{P}\left(\mathrm{V}=\left.0\right|_{\mathrm{L}=0}\right)=1-\mathrm{P}\left(\mathrm{V}=\left.1\right|_{\mathrm{L}=0}\right)=1-0.0209=0.9791 \\
& \mathrm{P}\left(\mathrm{V}=\left.1\right|_{\mathrm{L}=1}\right)=1-\mathrm{P}\left(\mathrm{V}=\left.0\right|_{\mathrm{L}=1}\right)=1-0.0385=0.9615 \\
& \mathrm{P}\left(\mathrm{L}=\left.0\right|_{\mathrm{T}=1}\right)=0.0018 \\
& \mathrm{P}\left(\mathrm{L}=\left.1\right|_{\mathrm{T}=0}\right)=0.0043 \\
& \mathrm{P}\left(\mathrm{L}=\left.0\right|_{\mathrm{T}=0}\right)=1-\mathrm{P}\left(\mathrm{L}=\left.1\right|_{\mathrm{T}=0}\right)=1-0.0043=0.9957 \\
& \mathrm{P}\left(\mathrm{L}=\left.1\right|_{\mathrm{T}=1}\right)=1-\mathrm{P}\left(\mathrm{L}=\left.0\right|_{\mathrm{T}=1}\right)=1-0.0018=0.9982 .
\end{aligned}
$$

Therefore, according to Equation 7-8,

$$
\mathrm{P}\left(\mathrm{V}=\left.1\right|_{\mathrm{T}=0}\right)=0.0043 \cdot 0.9615+0.9957 \cdot 0.0209=0.0249 \text {, }
$$

which is indeed close to the $P\left(V=\left.1\right|_{L=0}\right)$ value of 0.0209 . This can be seen in Figure 7-7 at 2:00 AM on the time scale. 


\section{Concluding Remarks}

After performing the necessary calculations for the likelihood analysis, there are several advantages of likelihoods that should be mentioned. The likelihood calculations are based on existing concepts of MOEs for the detection of discrete events. For this research, the existing MOEs were modified to accommodate presence detection. Another advantage of the likelihood approach is that sound econometric models and statistical software packages can be applied to the data in order to estimate the impacts of various factors of video detection performance.

Inductive loop output was used as reference data to evaluate video detection. This is acceptable as long as there is not a great deal of imperfection in the reference data. Using likelihood values, one is able to incorporate into the analysis the imperfection of the inductive loops, or any other device used as the reference. The imperfection of the loops can be introduced into long-term evaluation procedures at a low cost of limited human observations without the need for extensive ground truth data collection.

The likelihood models presented in this chapter indicate that the two video detection systems respond differently to different weather and traffic factors. Although it is difficult to say which system is better in general, it is possible to say which system performs better under what conditions. System 1 misses vehicle presence more than System 2 during the rain (Table 7-7). System 2 misses the most vehicle presence during the snow and sun (Table 7-9). This conclusion should be taken with caution, because neither sun nor snow is represented in the sample sufficiently.

According to the results of this research, and based only on a single installation, System 1 generates less false vehicle presence than System 2. There is no single factor in the System 1 model that degrades system 
performance over $5 \%$ from the base conditions (Table 7-8). System 2, on the other hand, tends to generate false vehicle presence over $24 \%$ of the time when the pavement is wet (Table 7-10).

Based upon the results of the evaluation performed for this report, recommendations can be made as to which system should be used, if any, for deployment. Strictly based on the results of the models presented in this chapter, and on observation of the performance of the video detectors on the text overlay of the saved video, it is has been concluded that System 1 operates with more stability than System 2. There are extended periods of time when System 2 will simply not detect vehicles in the left turn lanes. Over time, System 2 performance on Phase 6 degraded greatly. After the final recalibration, the performance of the video detector on this phase greatly improved, however.

Finally, in regards to problem documented in Figure 7-3, a test was conducted from 1am to 4am on October 26, 2001 with the assistance of the Crawfordsville district. During that test, the Southbound approach at Northwestern and Stadium was illuminated with a 400 Watt High Pressure Sodium Light mounted approximately $36^{\prime}$ in the air $18^{\prime}$ from the that base of the strainpole. Figure $7-8 \mathrm{a}$ shows an image of what the video camera observes without lighting and Figure $7-8 \mathrm{~b}$ shows an image of what the video camera observes with the lighting.

Figure 7-8c and Figure 7-8d show that with the lighting, even a vehicle with no headlights can be successfully detected. 


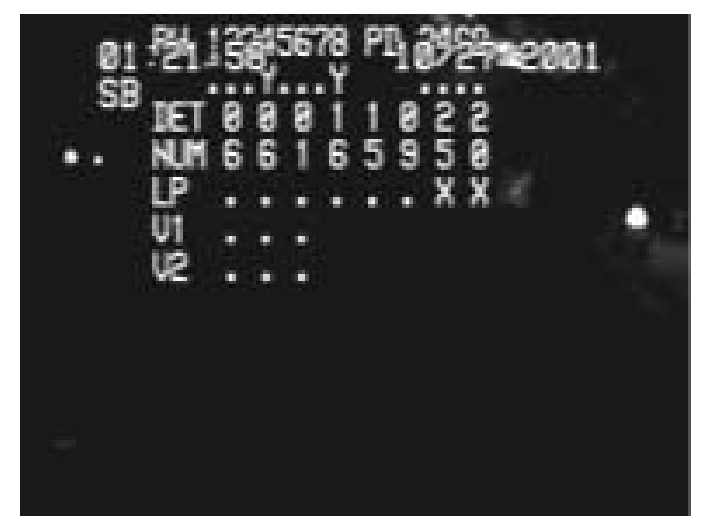

a) No lighting, no vehicles.

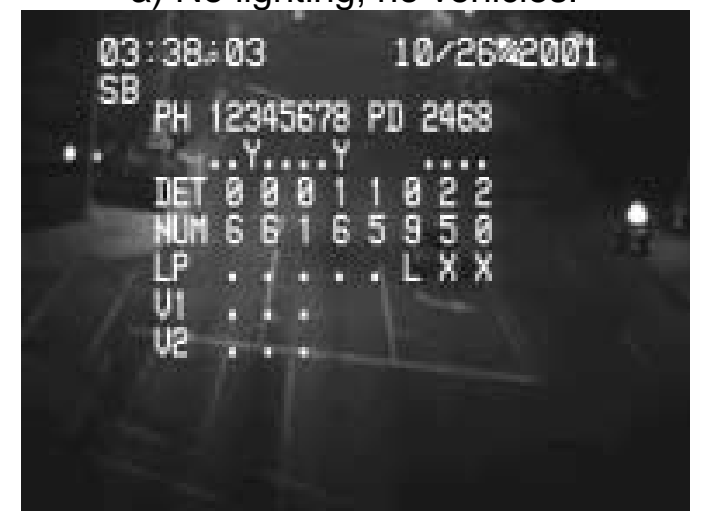

c) Lighted approach, vehicle with no lights approaching back loop (Detector 9)

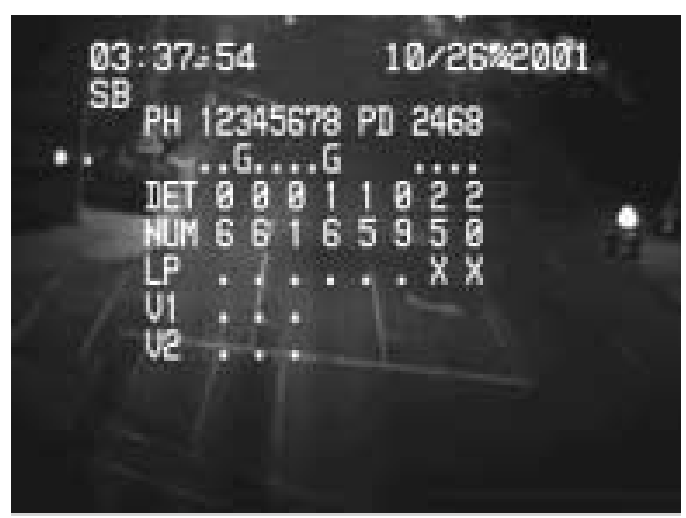

b) Lighted approach, no vehicles.

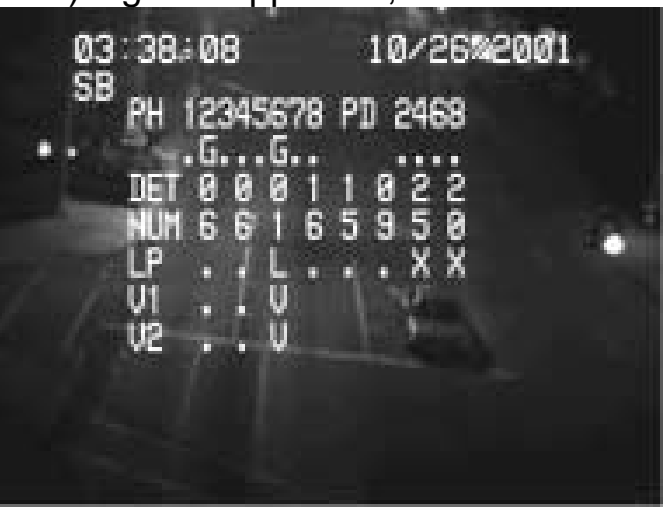

d) Vehicle with no lights successfully detected by loops and both video detectors (Detector 01)

Figure 7-8: Test with Southbound approach lighted with 400 Watt High Pressure Sodium Light 


\section{CHAPTER 8 - CONCLUSIONS}

In this report, there have been two different, and yet complimentary, types of evaluation for video detection performance demonstrated. It has been shown that the test facilities built and used for this research is capable of collecting the necessary data for video detection evaluation. Not only is it useful for evaluating the performance of video detection, but the test facility could also serve as a training facility at which technicians could be trained in how to set up and calibrate video detectors for optimum performance. If one were interested in learning how to calibrate video detection systems, this test facility could be very valuable, in that any changes in the video detectors can be deployed in a realworld situation without any control consequences. The test facility has been constructed in a way that an additional video detection system may be easily installed, should there be a need to evaluate further systems than those evaluated in this research.

Performing video detection evaluation with loop-video discrepancies, as done in Chapter 6, gives some quick insight into the performance of video detection with respect to inductive loop detectors. This approach should be used with caution, in that any large discrepancies should be investigated by viewing the saved digital video. This allows for the determination of whether the discrepancy is caused by the inductive loop or the video detector. This is the quickest of the two evaluation methods, but does not directly deal with the imperfection of inductive loop detectors. Because it is the quickest, it can be used in order to help train technicians in how to set up and maintain video detection systems. 
Using likelihoods, as done in Chapter 7, allows one to incorporate the effect of loop imperfection into the model. It also adds more time to the evaluation process in order to collect and process ground truth data for the inductive loops. Once this ground truth data collection is performed for the inductive loop; however, the loop imperfection can be easily inserted into the model of the likelihood of detection discrepancies. From this research, at this single intersection, the loop-video discrepancy model showed under the base conditions:

- System 2 was over twice more likely to have LOV1 discrepancy than System 1 (Table 7-7 and Table 7-9).

- System 1 and System 2 are both 7-8\% likely to have L1V0 discrepancy (Table 7-8 and Table 7-10).

- System 1 performs better.

Likewise, the loop-video discrepancy model showed that under the worstcase conditions:

- System 1 missed vehicle presence approximately $16 \%$ of the time, and generated false presence over $40 \%$ of the time.

- System 2 missed vehicle presence approximately $20 \%$ of the time, and generated false presence over $40 \%$ of the time, as well.

The calculation of likelihoods as presented in this report is a direct extension of existing MOEs for count detectors to presence detectors. Therefore, the methods demonstrated here are based on ideas that are already widely accepted. Another benefit of the likelihood calculations is that they can be used to model the effects of various weather and traffic characteristics on selected video detection systems.

The loop error model showed that the phase 5 loop rarely, if ever, misses vehicle presence. It did show that the loop had a slight tendency to generate 
false presence during periods of high volume. It is for this reason that the imperfection of loops should be factored into the evaluation of video detectors.

The technology available today allows for the collection of data that was not possible only several years ago. This is why most previous video detection evaluation efforts focused on accuracy of counts and speeds. This research goes a step beyond the common MOEs of count and speed, and shows how an inductive loop presence detector can be used to evaluate the presence detection capability of video detection systems as well.

Based upon this work, nighttime detection appears to be of the most concern. Two types of problems were observed:

- The effective length of the detection zone increased from an average of $23.7 \mathrm{ft}$ during the day to an average of $67.7 \mathrm{ft}$ at night. Figure 6-34 and Figure 6-35 document this phenomenon. This has a neglible impact on safety. However, signals operate less efficiently at night because they do not gap out when they should.

- Lost detection when vehicles pull past the stop bar. Figure 7-3 documents this phenomenon. Loop detectors typically do not lose a call in these situations, because the back of the vehicle is still in the proximity of the loop detection zone. However, video detection frequently only detects the headlights at night so the call is lost if the video detection zone ends just a few feet in front of the stop bar. Due to varying camera angles it is difficult to give an exact distance, but this type of failure can be mitigated by drawing the video detectors out in front of the stop bar several feet. However, judgment must be used when extending these detection zones because extending these detection zones often results in left turning vehicles or pedestrians generating false calls. This problem can also be addressed by lighting the intersection. Figure 7-8 documents the 
effectiveness of adding a 400-Watt High Pressure Sodium Light to the Southbound approach.

Based upon these observations, it is recommended that due to the imprecision of night time detection (Figure 6-33 and Figure 6-34), video detection should not be used to provide dilemma zone protection. The imprecision observed at the stop bar is even worse at the extended distance at which dilemma zone detectors are placed.

In addition, it is recommended that the turn on testing procedure at all new installations encompass the following to ensure that vehicle calls are not lost by video detectors during low volume at night and detectors do not have an unacceptably high false detection rate:

- Pick a low volume time to test - say 4am.

- Ensure that no other vehicles are in the vicinity of the signal.

- Drive test car into lane being evaluated. Via radio, the inspector at the cabinet shall communicate to the driver when the detector being evaluated turns on and turns off.

- Confirm that detection is registered by video detector when vehicle approaches detection zone.

- Creep car forward until detection is lost. Record how many ft the front bumper was past the stop bar. Record in acceptance notes and note on cabinet plans.

- Repeat process for all lanes with video detection.

- After nighttime test and adjustments are complete, conduct daytime test to ensure that left turning vehicles and pedestrians are not causing an unacceptably high rate of false calls. Note, it is important that this daytime test follow the nighttime testing. 
Finally, although not directly part of the scope of this project, the instrumented intersection at Northwestern and Stadium provided a mechanism for conducting the count detector tests documented in Appendix A. Based upon the results of the final tests, the Reno detector demonstrated as good if not better performance then the current "fourth loop" for counting cars. If multiple vendors can achieve similar levels of performance, consideration should be given to abandoning the practice of wiring the "fourth loop" to a different detection channel and instead use the new count detector cards.

A last minute addition to this study was to evaluate the accuracy of counts with video detection systems. Appendix B contains a memorandum explaining the procedure used and suggests that turning movement counts obtained from video detection systems are not sufficiently accurate for designing signal timings. 


\section{LIST OF REFERENCES}




\section{LIST OF REFERENCES}

Cottrell, B. H. Jr. "Evaluation of a Video Image Detection System (VIDS) - Final Report," Research Report No. VTRC 94-R22, Virginia Transportation Research Council, Virginia Department of Transportation, Charlottesville, VA, May 1994.

MacCarley, C. A., S. L. M. Hockaday, D. Need, and S. Taff. "Evaluation of Video Image Processing Systems for Traffic Detection," Transportation Research Record No. 1360, Transportation Research Board, Washington, D.C., 1992.

MacCarley, A., "City of Anaheim/Caltrans/FHWA Advanced Traffic Control System Field Operational Test Evaluation: Task C Video Traffic Detection System - Final Report," Research Project RTA 65V313-4, Research Report No. UCB-ITS-PRR-98-32, California Partners for Advanced Transit and Highways, California Polytechnic State University, San Luis Obispo, CA, September 1998.

Middleton, D., D. Jasek, and R. Parker, "Evaluation of Some Existing Technologies for Vehicle Detection," Research Project FHWA/TX00/1715-S, Research Report 1715-S, Texas Transportation Institute, College Station, TX, September 1999.

Middleton, D., R. Parker, "Initial Evaluation of Selected Detectors to Replace Inductive Loops on Freeways," Research Project FHWA/TX-00/1439-7, Research Report 1439-7, Texas Transportation Institute, College Station, TX, April 2000.

Minnesota Department of Transportation - Minnesota Guidestar and SRF Consulting Group, "Field Test of Monitoring of Urban Vehicle Operations Using Non-Intrusive Technologies," Volume 5, Task Three Report: Extended Field Test Results, Minnesota Guidestar, St. Paul, MN, and SRF Consulting Group, Minneapolis, MN, December 1996.

Orcutt, Fred L., P.E., The Traffic Signal Book. Englewood Cliffs, NJ: Prentice Hall. 1993. p. 67. 
APPENDICES 


\section{APPENDIX A - SUMMARY OF COUNT DETECTOR EVALUATION}




\title{
Purdue University
}

March 3, 2001

\author{
Lauri Land \\ Procurement and Distribution \\ Traffic Support \\ 6400 East 30th Street \\ Indianapolis, IN 46219 \\ Dear Lauri:
}

As you are aware, we have instrumented the intersection of U.S. 231 and Stadium Avenue in West Lafayette, IN for the purpose of testing loop and video detection systems. Figure A-1 shows a diagram of the intersection and detector layout. Figure A2 shows a block diagram of the instrumentation, Figures A-3-A-5 show photographs of the cabinets at the intersection. Figure A- 6 shows monitoring equipment located in CIVL 1122. Figure A-7 shows how the "counts" from both the traditional back loop (Detectors 1,2 , and 3 for the Northbound direction) and the new "count" outputs (Detectors 4, and 5 for the Northbound direction are recorded on the video. Figures A-8 - A-10 show the detector arrangement for Southbound, Eastbound, and Westbound cameras respectively.

Preliminary count detector tests were conducted during the Spring of 2000 . We performed a second round of testing of the Reno Count Detector in October 2000. On October $12^{\text {th }}, 2000$ we conducted tests in the Northbound, Southbound, and Eastbound lanes. We did not evaluate the Westbound lanes as there is a driveway adjacent to the loops and vehicles frequently drive over the loops perpendicular to the travel direction. Table A-1 summarizes the performance of that test, and you were previously supplied with a CD containing video files documenting that test. Based upon this limited test, I offered the following comments in my October 25, 2000 letter report:

- In general, the count detectors did better then the back loops, with the exception of the "SL" detector. However, given the relatively small number of counts observed, even the $17.54 \%$ error may be a bit misleading.

- Cross talk may be an issue with the back loop detectors performing poorly. We should probably meet to further review this issue.

$3 \mathrm{M}$ performed a second round of tests on September 9, 2000. However, since those cards did not have the contact closure output $3 \mathrm{M}$ provided equipment for polling the cards via the detector serial port and overlaying that data on the image. Since it was there equipment, extended tests were not possible. In my October 25, 2000 letter report I had chosen not to report those results due to the very short test. However, at the request of Bob Dreger, I am reporting that information in Table A-2. Since it is a very short sample, you should be cautious in interpreting the relative error. 
A Reno factory representative visited the site on December $18^{\text {th }}, 2000$. He examined the status of the loops and further tuned the configuration. We repeated the October protocol on February $28^{\text {th }}, 2001$ and those results are summarized in Table A-3. A CD accompanying this letter has a digital video documenting the test. Also, during his visit, the Reno representative recorded several wave forms from the loops. Those are shown in Figures $A-11-A-15$.

Regarding the performance shown in Table A-3, we offer the following comments:

- In general, the count detectors did better then the back loops, with the exception of the "SL" detector.

- The count detectors appeared to perform better for the through movements then the left turn lanes (Examine NA, SA relative error vs. NL, SL, or EL).

Based upon the graphs shown in Figures $A-11-A-15$, the following comments were offered by the Reno factory representative. Where possible, we have attempted to offer quantitative data to support or question those comments.

- Figure A-11 illustrates the near perfect progression of one vehicle across the loop. This type of signature is indicative that the system will perform well. This information appears to be substantiated by the performance of the SA front count loop detector (1.9\% and $-1.5 \%$ in Tables $A-1$ and $A-3$ respectively).

- Figure A-12 illustrates a signature of a vehicle where the factory representative observed a path across all loops, but the first loop recorded a relatively low change in inductance. The representative suggested this was most likely due to their only being three turns in the rear loop. This information appears to be substantiated by the relatively poor performance of the SL front count loop detector (17.5\% and $10.6 \%$ in Tables A-1 and A-3 respectively). However, since we do not have video of the vehicle path, we can not be certain that it was not the result of poor lane following by the driver.

- Figure A-13 illustrates the signature of a vehicle where the factory representative observed the vehicle moving off the loop due to snow cover in the lane. No tests were conducted during snow, but I thought this signature was good to show the impact of drivers demonstrating poor lane discipline.

- Figure A-14 illustrates the signature where the factory representative observed a vehicle path across all loops. It is not quite as good as that shown in Figure A11 , most likely due vehicles not traversing the center of all loops. This information is not completely substantiated by the performance of the front count detector (-3.6\% and $-13.0 \%$ in Tables $A-1$ and $A-3$ respectively).

- Figure A-15 illustrates the signature of one vehicle proceeding across the eastbound detectors where the vehicle turns off short of clearing the front detector (note: Figure A-1 does not perfectly illustrate the detector location as the left turn detectors extend past the stop bar in the field.). This type of error has the potential to be significant for many left turn pockets. Perhaps one explanation of the differences in performance of the EL detector in Tables A-1 and $A-3$ is that different proportion of vehicles turned short of clearing the front detector loop.

- The relatively poor performance of the traditional back loop $(7.6 \%, 26.8 \%$, $29.3 \%, 28.7 \%,-2.1 \%, 9.6 \%, 47.7 \%, 33.6 \%$ for NB, NA, NL, SA, SL, EB, EA, and EL in Table A-3) could probably be improved if detector cards were used in count mode. No tests were conducted to verify this, but based upon the discussion of 
the waveforms shown in Figures $A-11-A-15$, I believe this issue may worth pursuing further.

In summary, the Reno detectors provided less then $3 \%$ relative error for the two through movements tested this time (NA, SA in Table A-3). However, the performance of the Reno cards for the left turn movements was noticeably higher $(5.4 \%, 10.6 \%,-13.0 \%$ for NL, SL, EL in Table A-3).

Finally, in conclusion, both Reno and 3M representatives have indicated their algorithm would perform better with four loops in series and would like their equipment evaluated in that configuration. I would propose we conduct one final test with the NA, NL, SA, SL, and EL lanes configured with four loops in series. Such a test will require temporary lane closures for an INDOT crew or contractor to splice that fourth loop into the appropriate circuit.

Should you have any questions, please contact me at 765/494-2226.

Sincerely,

Darcy Bullock

Associate Professor 


\begin{tabular}{|c|cccc|}
\hline & \multicolumn{4}{|c|}{ Sunny Conditions } \\
\hline Northbound 09:00 - 10:00 10/12/00 & Hours & Loop Count & Human Count & $\%$ Error \\
NB, Detector 1 (Back) & 1 & 232 & 213 & 8.92 \\
NA, Detector 2 (Back) & 1 & 148 & 123 & 20.33 \\
NL, Detector 3 (Back) & 1 & 257 & 190 & 35.26 \\
NA, Detector 4 (Front Detector) & 1 & 133 & 122 & 9.02 \\
NL, Detector 5 (Front Detector) & 1 & 201 & 190 & 5.79 \\
\hline Southbound 11:00 - 12:00 10/12/00 & & & & \\
SA, Detector 1 (Back) & 1 & 373 & 263 & 41.83 \\
SL, Detector 2 (Back) & 1 & 62 & 58 & 6.90 \\
SA, Detector 3 (Front Detector) & 1 & 268 & 263 & 1.90 \\
SL, Detector 4 (Front Detector) & 1 & 67 & 57 & 17.54 \\
\hline Eastbound 13:00 - 14:00 10/12/00 & & & & \\
EB, Detector 1 (Back) & 1 & 374 & 348 & 7.47 \\
EA, Detector 2 (Back) & 1 & 268 & 210 & 27.62 \\
EL, Detector 3 (Back) & 1 & 197 & 166 & 18.67 \\
EL, Detector 4 (Front Detector) & 1 & 159 & 165 & -3.64 \\
\hline
\end{tabular}

Table A-1: Summary of Reno Detector Performance, October 2000

\begin{tabular}{|c|cccc|}
\hline & \multicolumn{4}{|c|}{ Sunny Conditions } \\
\hline $\begin{array}{c}\text { Northbound 14:30-14:45 9/9/2000 } \\
\text { NA, Detector 4 (Front Detector) }\end{array}$ & Hours & Loop Count & Human Count & $\%$ Error \\
Northbound 15:12 - 15:27 9/9/2000 & & 38 & 56 & $-32 \%$ \\
NL, Detector 5 (Front Detector) & 0.25 & 52 & 62 & $-16 \%$ \\
\hline $\begin{array}{c}\text { Southbound 15:45 - 15:55 9/9/2000 } \\
\text { SA, Detector 3 (Front Detector) }\end{array}$ & 1 & 74 & 45 & $+64 \%$ \\
\hline
\end{tabular}

Table A-2: Summary of 3M Detector Performance, September 2000 


\begin{tabular}{|c|cccc|}
\hline & \multicolumn{4}{|c|}{ Sunny Conditions } \\
\hline Northbound 08:00:29 - 09:00:00 2/28/01 & Hours & Loop Count & Human Count & $\%$ Error \\
NB, Detector 1 (Back) & 1 & 212 & 197 & 7.61 \\
NA, Detector 2 (Back) & 1 & 123 & 97 & 26.80 \\
NL, Detector 3 (Back) & 1 & 309 & 239 & 29.29 \\
NA, Detector 4 (Front Detector) & 1 & 100 & 97 & 3.09 \\
NL, Detector 5 (Front Detector) & 1 & 252 & 239 & 5.44 \\
\hline Southbound 09:00:30 - 10:00:00 2/28/01 & & & & \\
SA, Detector 1 (Back) & 1 & 332 & 258 & 28.68 \\
SL, Detector 2 (Back) & 1 & 46 & 47 & -2.13 \\
SA, Detector 3 (Front Detector) & 1 & 254 & 258 & -1.55 \\
SL, Detector 4 (Front Detector) & 1 & 52 & 47 & 10.64 \\
\hline EB, Detector 1 (Back) & 1 & & & \\
EA, Detector 2 (Back) & 1 & 192 & 130 & 47.69 \\
EL, Detector 3 (Back) & 1 & 175 & 131 & 33.59 \\
EL, Detector 4 (Front Detector) & 1 & 114 & 131 & -12.98 \\
\hline
\end{tabular}

Table A-3: Summary of Reno Detector Performance, February 2001 


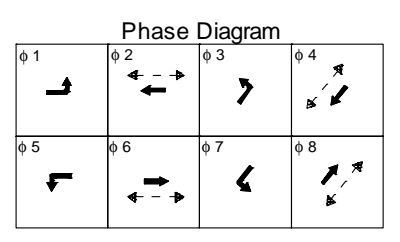

U.S. 231/ Northwestern Ave.
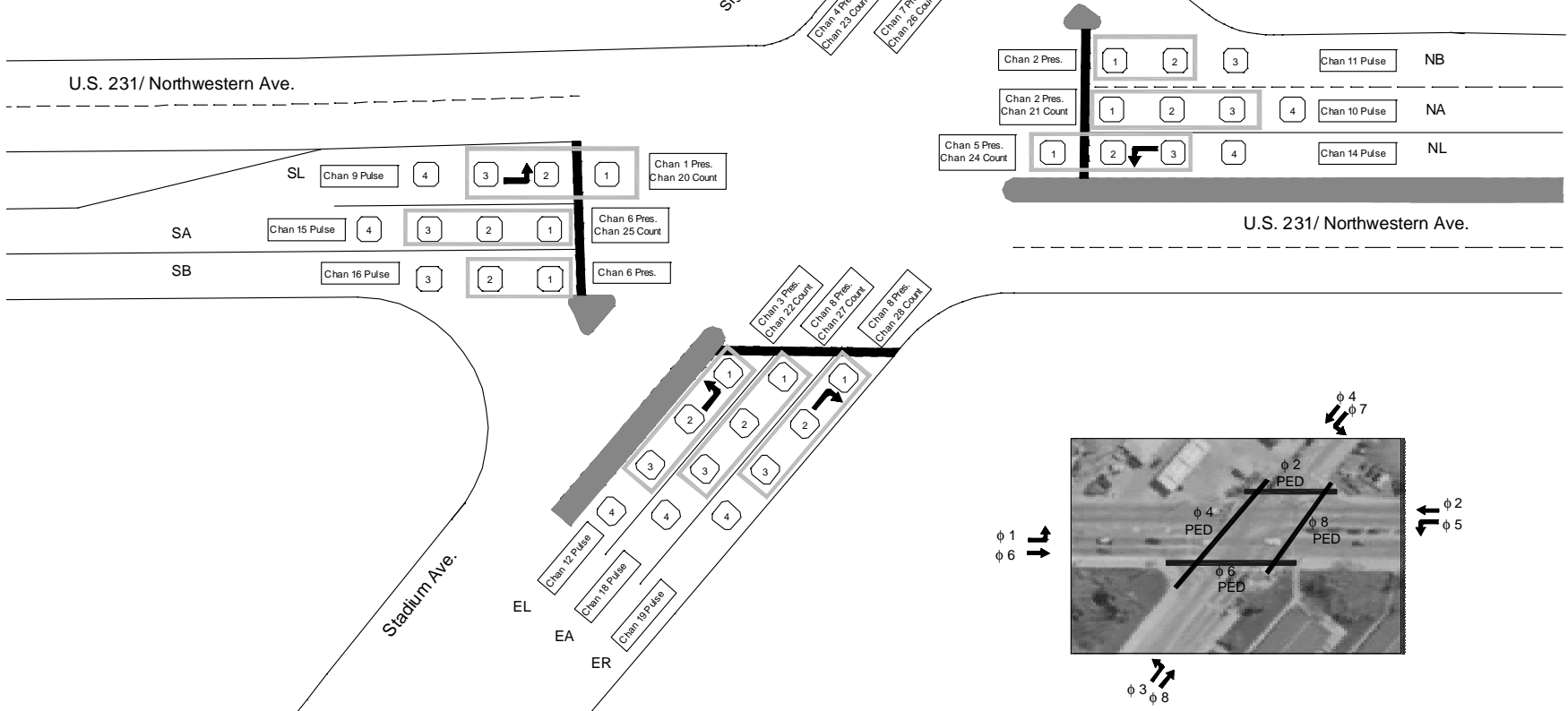

Figure A-1: Intersection and Inductive loop Layout

School of Civil Engineering • 4115 Civil Engineering Building • West Lafayette, IN 47907-1284

Phone: 765/494-2226 • FAX: 765/496-1105 • Email: darcy@purdue.edu 


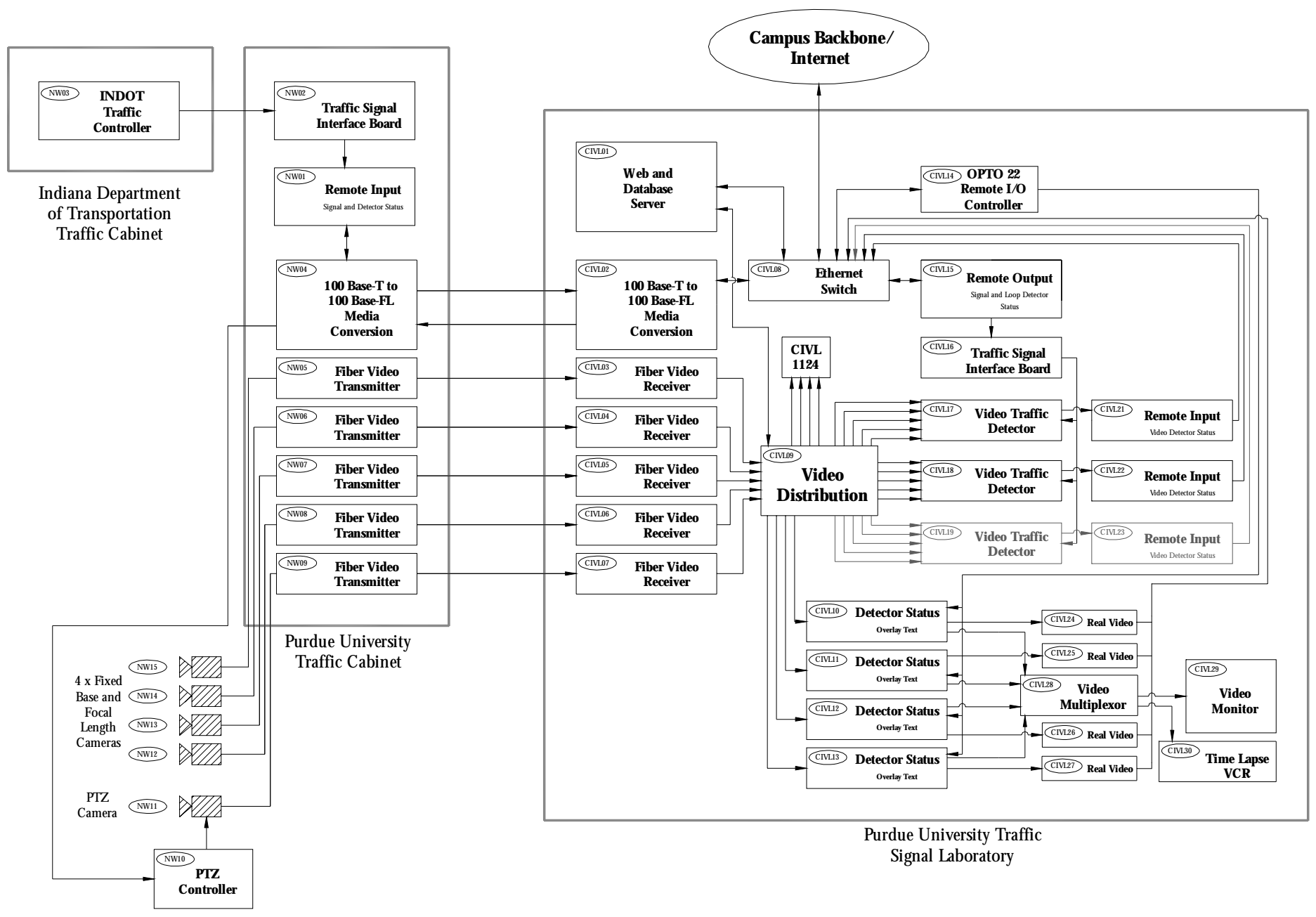

Figure A-2: Schematic Drawing of Test Bed Components

School of Civil Engineering • 4115 Civil Engineering Building • West Lafayette, IN 47907-1284 Phone: 765/494-2226 • FAX: 765/496-1105 • Email: darcy@purdue.edu 


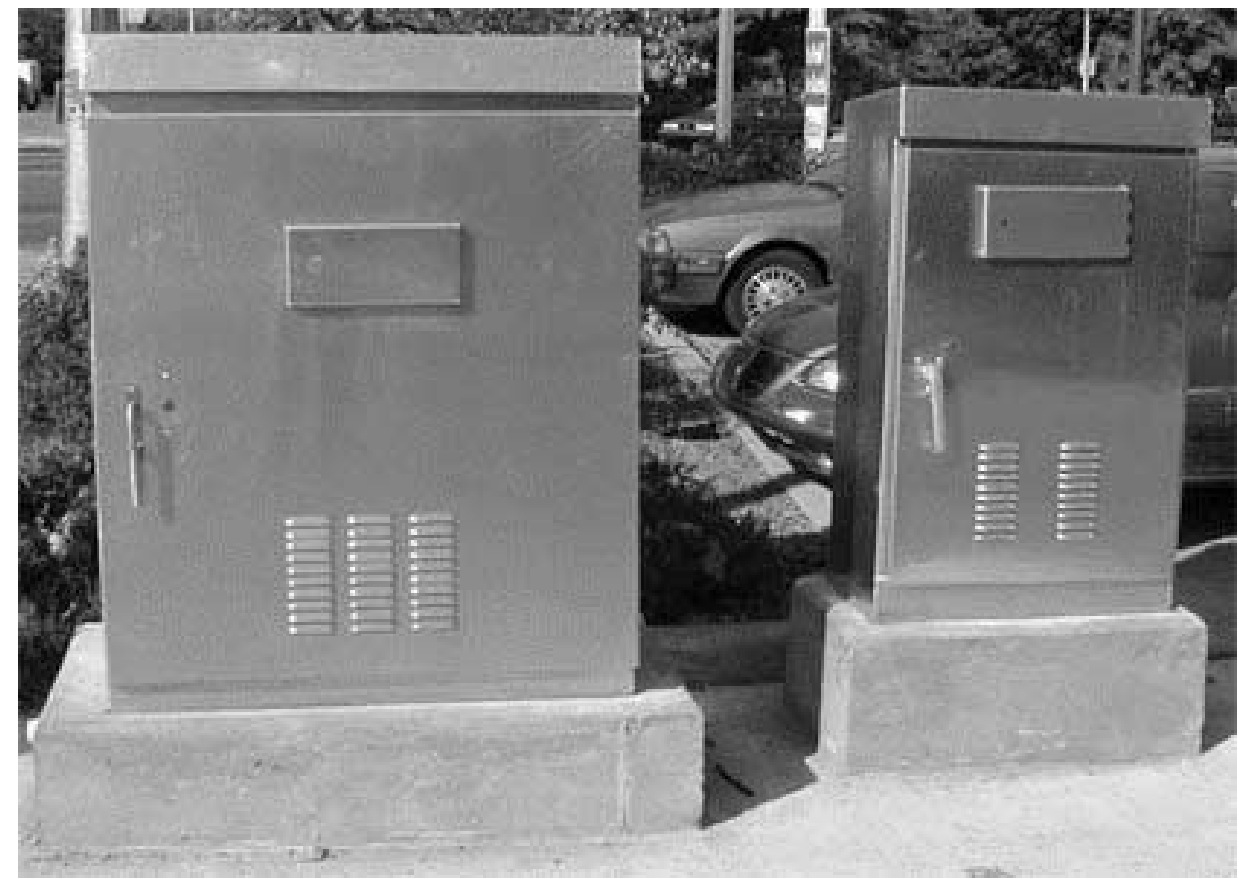

Figure A-3: INDOT and Purdue Cabinets

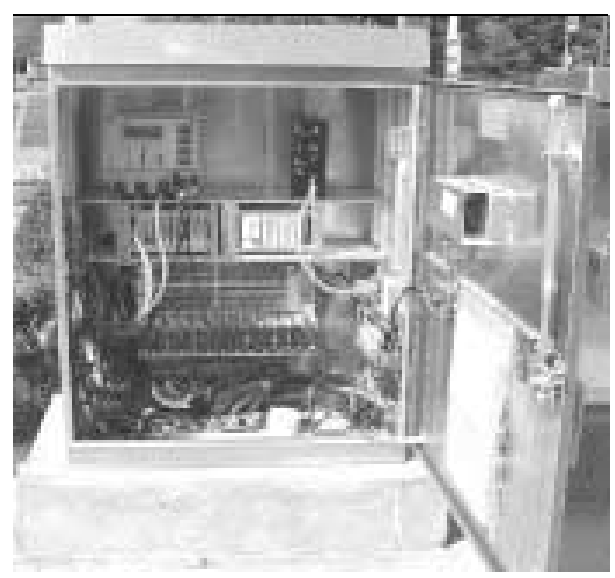

Figure A-4: INDOT Traffic Cabinet (NW03)

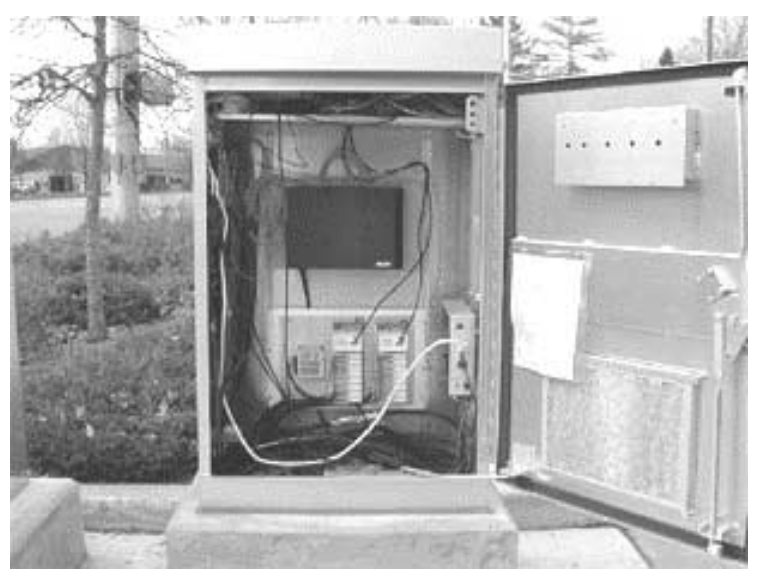

Figure A-5: Purdue Cabinet 


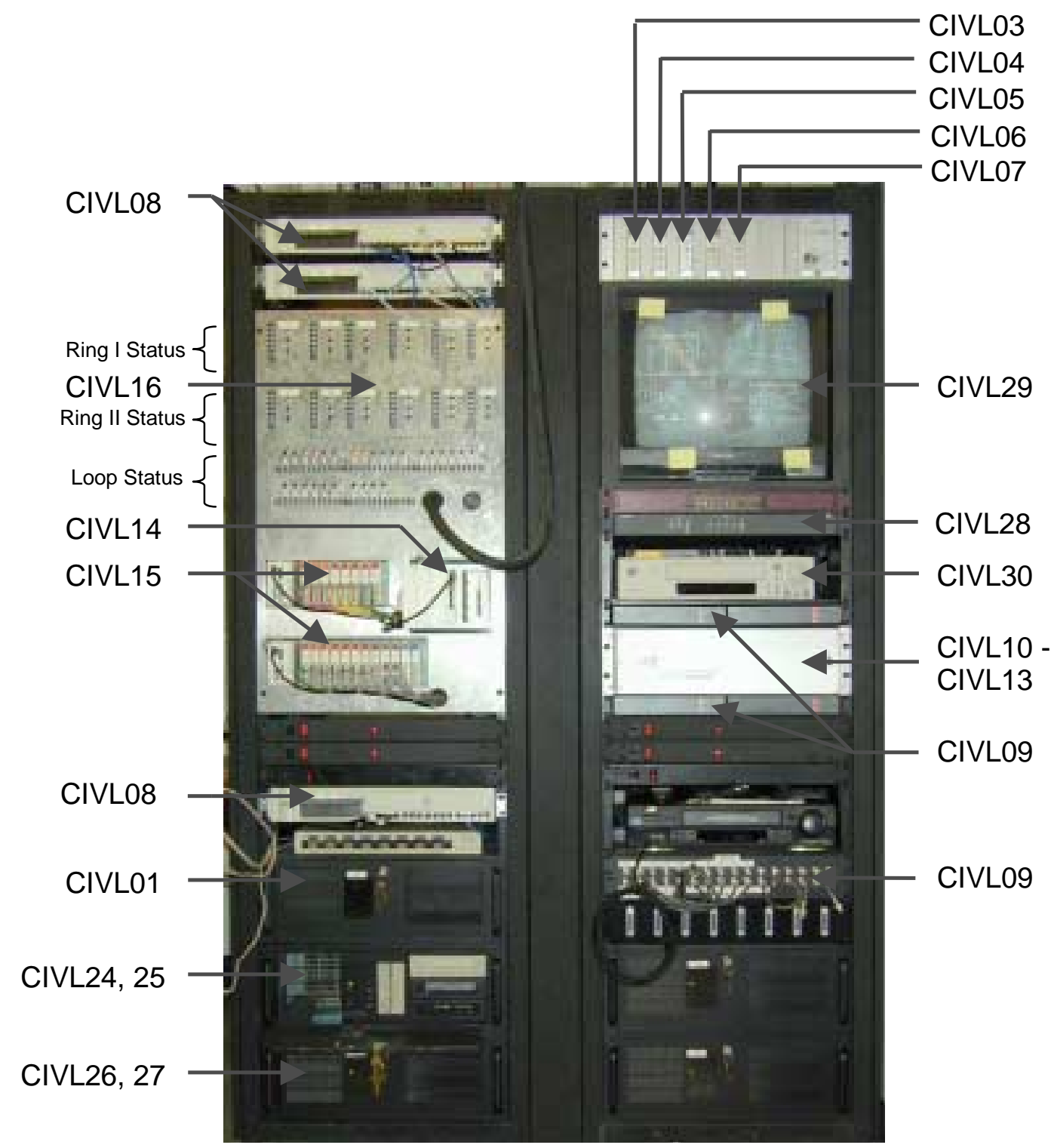

Figure A-6: Video and CID Interface in the Lab 


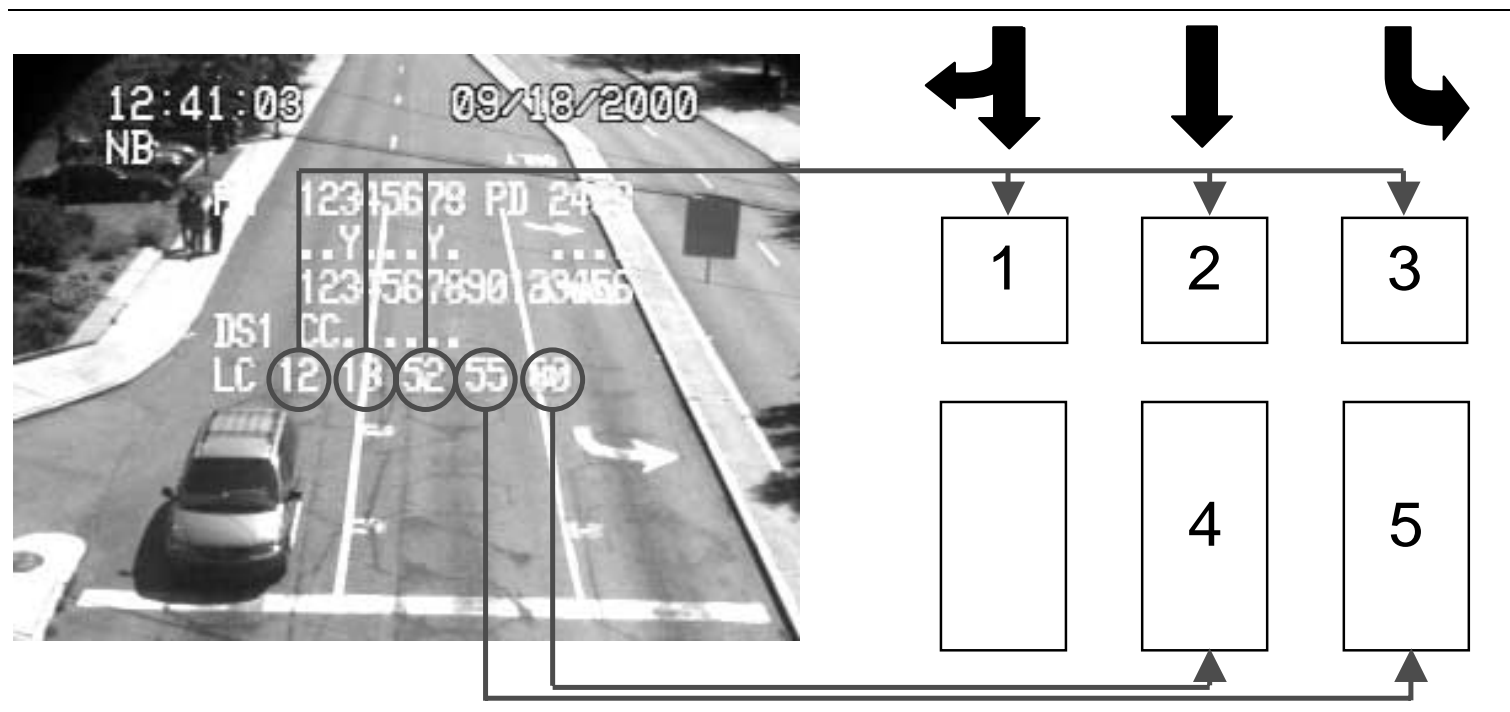

Figure A-7: Relation of text overlay to Northbound detection fields
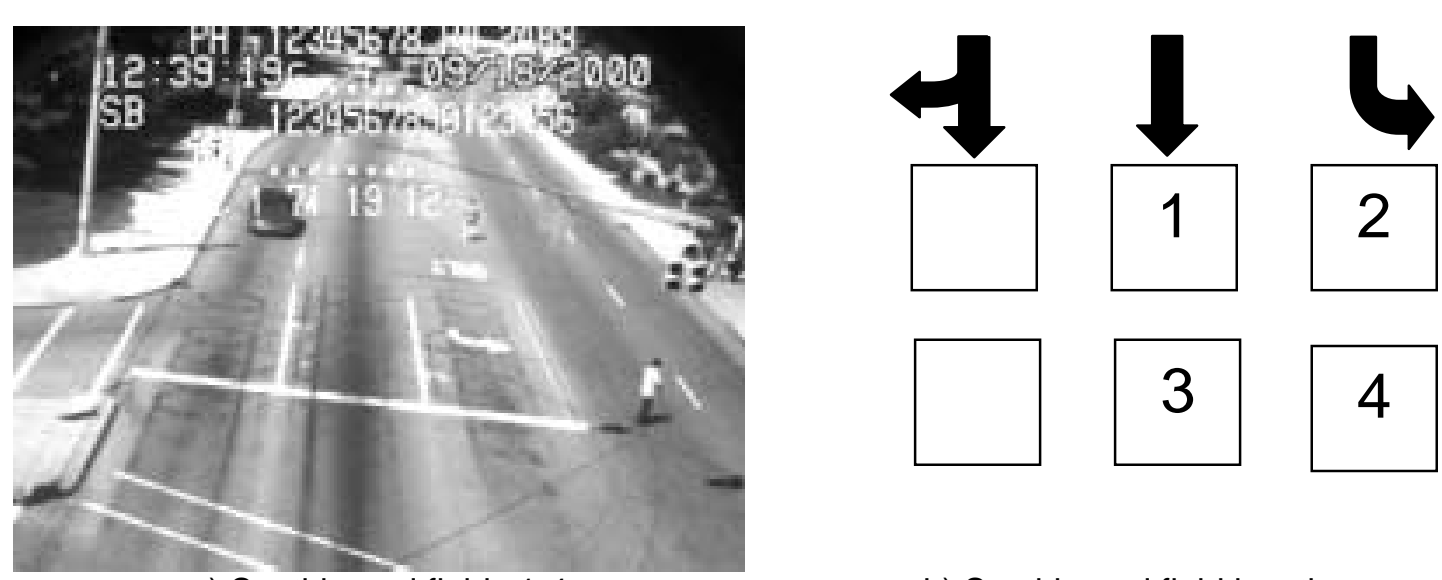

a) Southbound fields 1-4

b) Southbound field locations

Figure A-8: Southbound Fields 


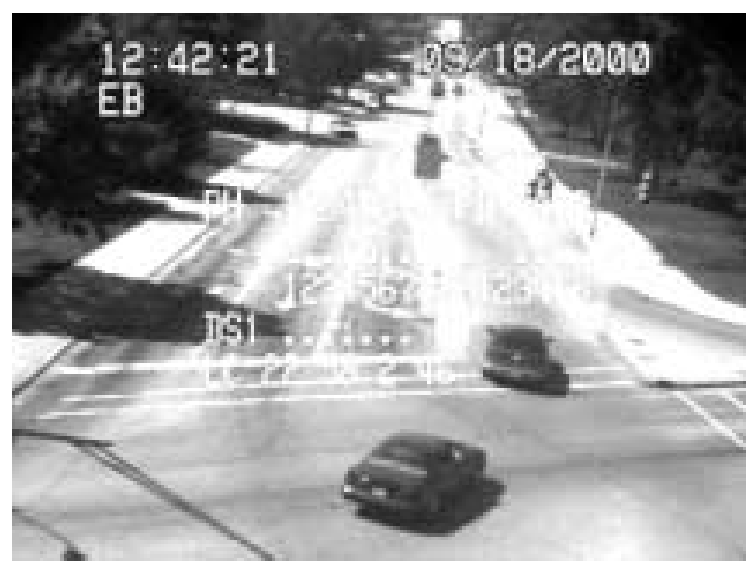

a) Eastbound fields 1-4
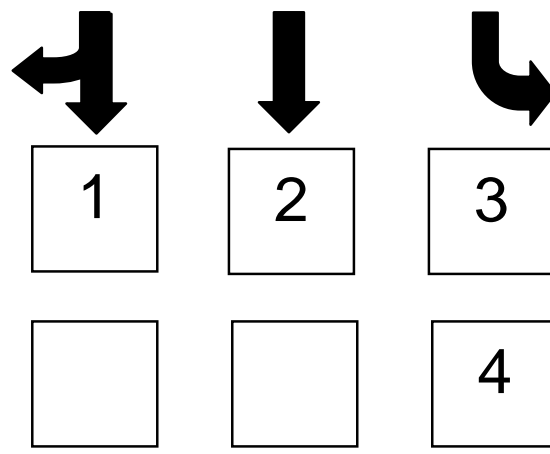

4

Figure A-9: Eastbound Fields

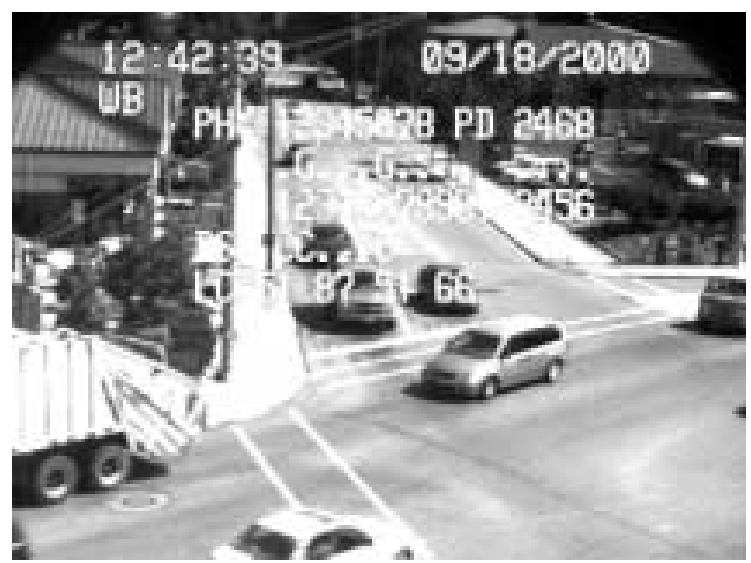

a) Westbound fields 1-4
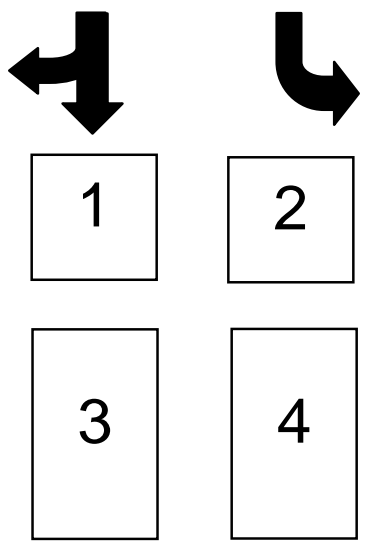

b) Westbound field locations

Figure A-10: Westbound Fields 


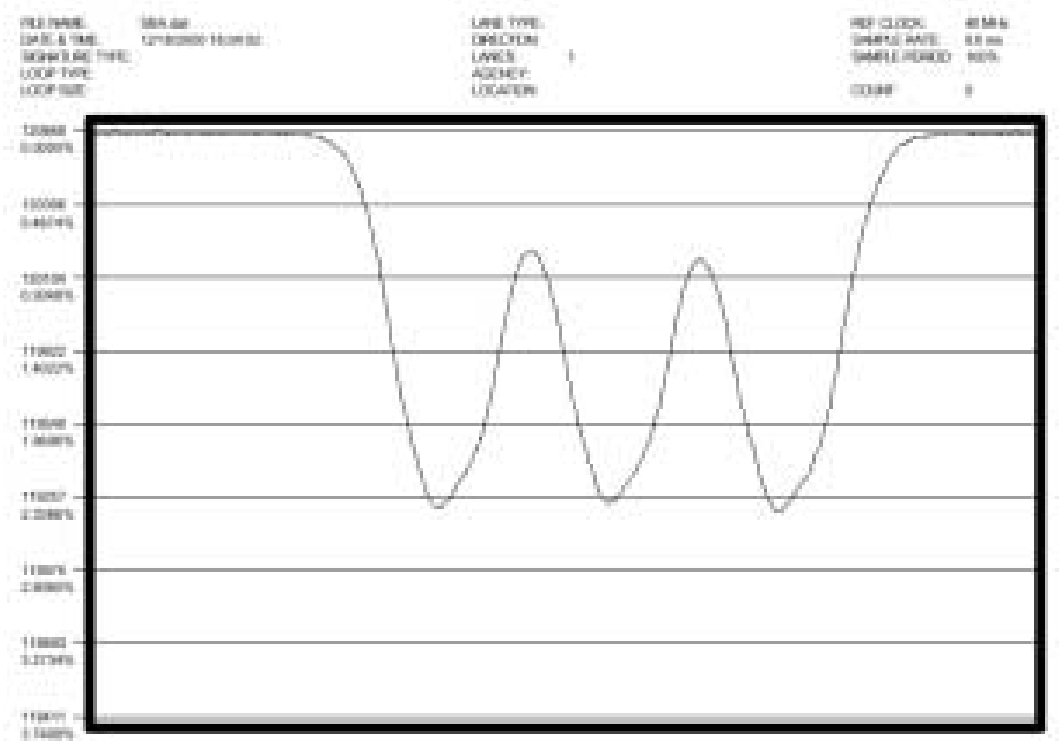

Figure A-11: Signature of one vehicle proceeding across south bound center lane loop.

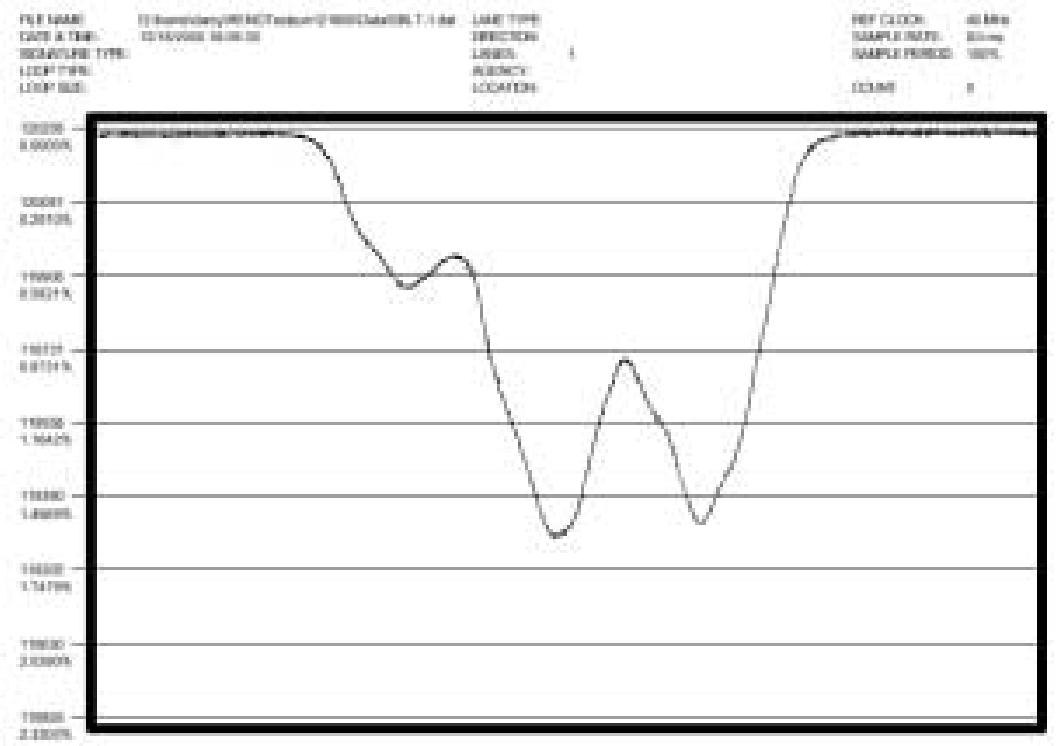

Figure A-12: Signature of one vehicle proceeding across south bound left turn with back loop perhaps missing a turn. 


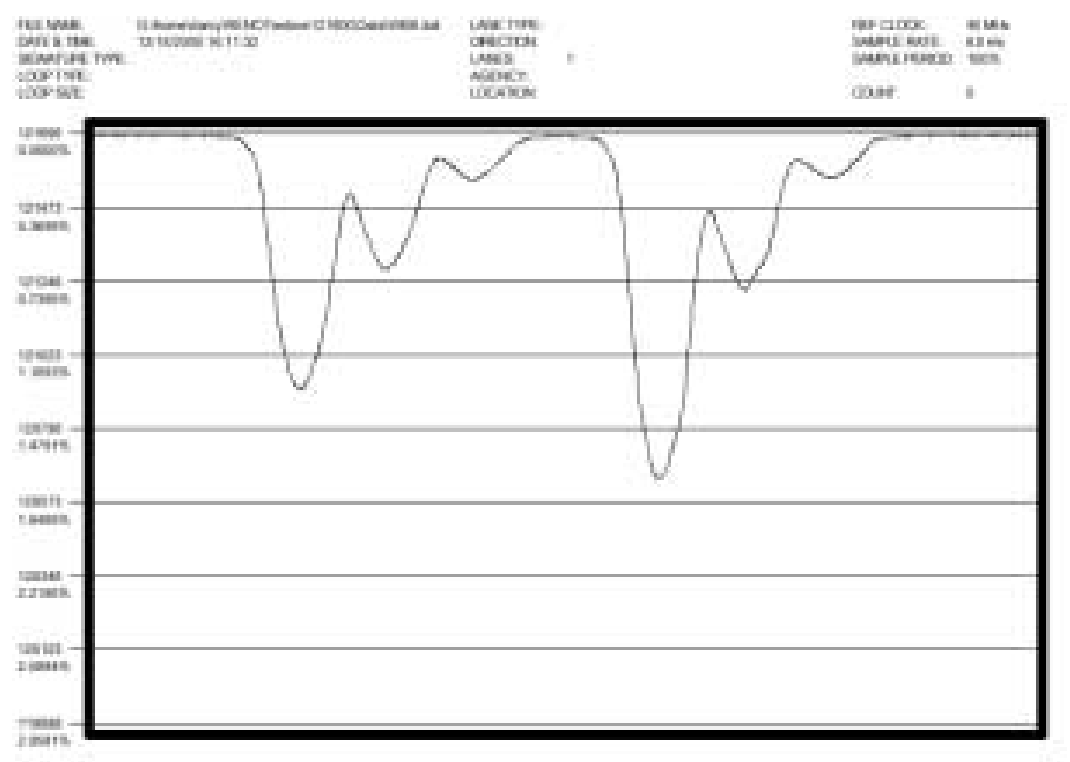

Figure A-13: Signature of two vehicles proceeding across west bound through movement moving off loop due to snow cover.

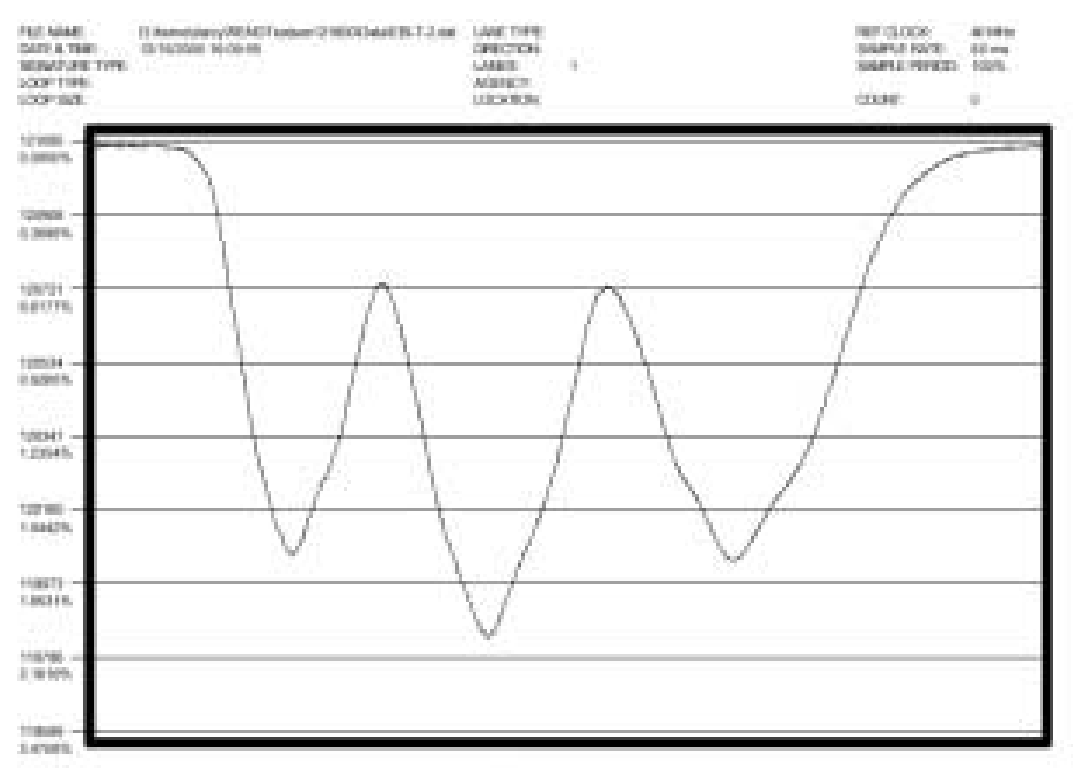

Figure A-14: Signature of one vehicle proceeding across all loops in east bound left turn pocket. 


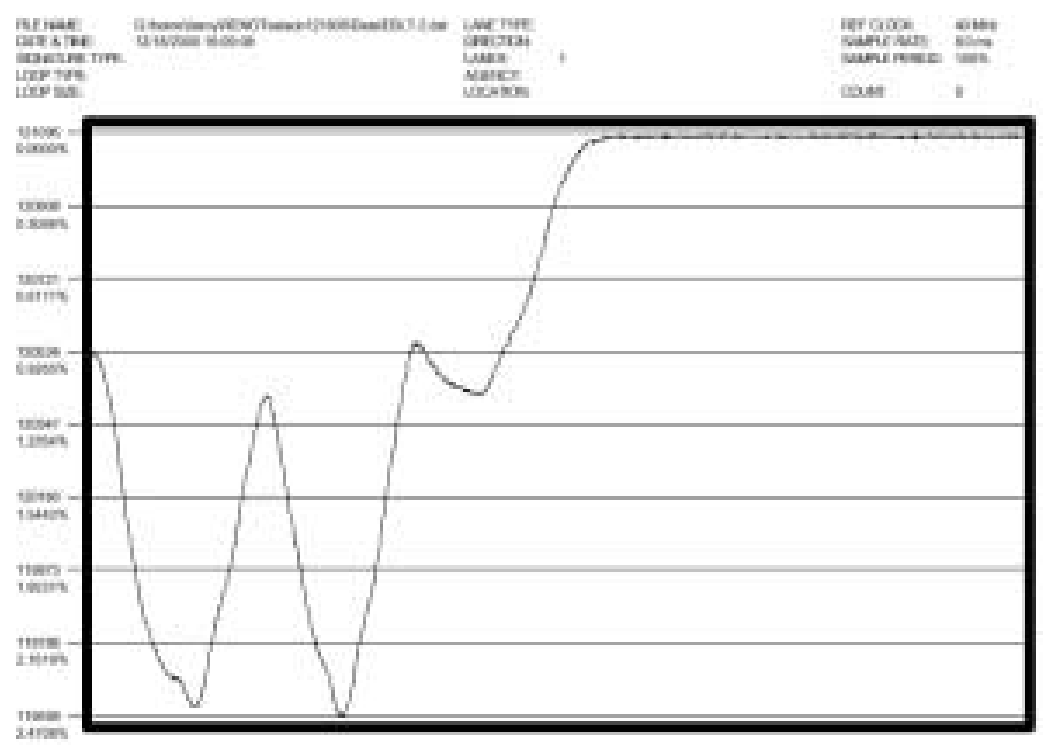

Figure A-15: Signature of one vehicle proceeding across loops in east bound left turn pocket, but turning before completely traversing the last loop 


\section{APPENDIX B - EVALUATION OF VIDEO DETECTION COUNT ACCURACY}


To:

From:

Date:

Subject:
Jim Sturdevant

Darcy Bullock, Andrew Nichols

October 31, 2001

Evaluation of Video Detection Count Accuracy

At the request of Mike Bowman and yourself on October 11, 2001, a study was conducted to evaluate the accuracy of vehicle counts using video detection. The Econolite Autoscope and Peek VideoTrak systems were used in the study. Both systems were programmed to output the count pulses to a high speed digital i/o module. This module was used to log the counts from each system. The actual counts were obtained from a student watching a video that was recorded and visually counting the cars in each lane.

The first series of tests were conducted between Sunday, 10/14/01 and Wednesday 10/17/01. Four time periods were recorded that consisted of weekend light traffic, weekday morning, weekday midday, and weekday evening. The weekday morning period was began before sunrise (dark conditions) and lasted approximately 20 minutes afterward. These results are shown in Tables 1 and 2 and Figures 1 through 4.

The results of these tests were given to the vendors of the two video detection systems and they were given an opportunity make adjustments. The VideoTrak vendor made adjustments on Tuesday 10/23/01 and the Autoscope vendor made adjustments on Thursday 10/25/01.

The second series of tests were conducted on Friday 10/26/01. Two time periods were recorded that consisted of weekday midday and weekday evening. The weekday evening time period began before sunset, but had approximately 15 minutes of dark conditions at the end. These results are shown in Tables 3 and 4 and Figure 5 and 6.

There was a noticeable improvement in the performance of both systems between tests 1 and 2. Although the Autoscope counts were closest to the observed counts, neither system produced turning movement counts that I would consider adequate for designing signal timing plans.

Should you have any questions, feel free to contact me at 765/494-2226 or darcy@Purdue.edu. 


\section{VIDEO DETECTION TEST 1}

Table 16: Test 1 Video Detection Counts

\begin{tabular}{|c|c|c|c|c|c|c|c|c|c|c|c|c|}
\hline \multirow{3}{*}{$\begin{array}{c}\text { Lane } \\
\text { NBLT }\end{array}$} & \multicolumn{3}{|c|}{$\begin{array}{c}\text { Weekend Light Traffic } \\
\text { Sunday } 10 / 14 / 01 \\
\text { 1000AM to } 1100 \mathrm{AM} \\
\text { Overcast }\end{array}$} & \multicolumn{3}{|c|}{$\begin{array}{c}\text { Weekday AM } \\
\text { Tuesday } 10 / 16 / 01 \\
615 A M \text { to } 717 \mathrm{AM} \\
\text { Sunrise, Rain }\end{array}$} & \multicolumn{3}{|c|}{$\begin{array}{c}\text { Weekday Midday } \\
\text { Wednesday } 10 / 17 / 01 \\
1145 \text { AM to } 1245 P M \\
\text { Sunny }\end{array}$} & \multicolumn{3}{|c|}{$\begin{array}{c}\text { Weekday PM } \\
\text { Wednesday } 10 / 17 / 01 \\
\text { 430PM to 530PM } \\
\text { Sunny }\end{array}$} \\
\hline & \multicolumn{3}{|c|}{ Autoscope VideoTrak Actual } & \multicolumn{3}{|c|}{ Autoscope VideoTrak Actual } & \multicolumn{3}{|c|}{ Autoscope VideoTrak Actual } & \multicolumn{3}{|c|}{ Autoscope VideoTrak Actual } \\
\hline & 112 & 167 & 96 & 198 & 302 & 120 & 379 & 551 & 210 & 427 & 610 & 254 \\
\hline NBLL & 132 & 119 & 115 & 131 & 237 & 80 & 24 & 375 & 210 & 422 & 538 & 361 \\
\hline NBRL & 126 & 166 & 129 & 129 & 169 & 100 & 328 & 313 & 265 & 525 & 659 & 463 \\
\hline SBLT & 35 & 31 & 25 & 51 & 51 & 33 & 10 & 17 & 71 & 156 & 131 & 53 \\
\hline SBLL & 200 & 217 & 183 & 302 & 487 & 125 & $2 \varepsilon$ & 101 & 256 & 351 & 428 & 348 \\
\hline SBRL & 108 & 111 & 109 & 197 & 245 & 97 & 15 & 855 & 126 & 193 & 188 & 175 \\
\hline EBLT & 27 & 152 & 101 & 55 & 92 & 3 & 25 & 527 & 18 & 1 & 896 & 328 \\
\hline EBLL & 79 & 175 & 87 & 62 & 169 & 4 & $3 \varepsilon$ & 638 & 193 & 315 & 521 & 309 \\
\hline EBRL & 171 & 149 & 114 & 132 & 142 & 59 & 39 & 640 & 306 & 603 & 1137 & 510 \\
\hline WBLT & 18 & 29 & 22 & 27 & 161 & 16 & 207 & 103 & 46 & 50 & 180 & 47 \\
\hline WBRL & 126 & 96 & 102 & 235 & 94 & 114 & 372 & 183 & 186 & 745 & 129 & 278 \\
\hline
\end{tabular}

Table 17: Test 1 Video Detection Error Summary

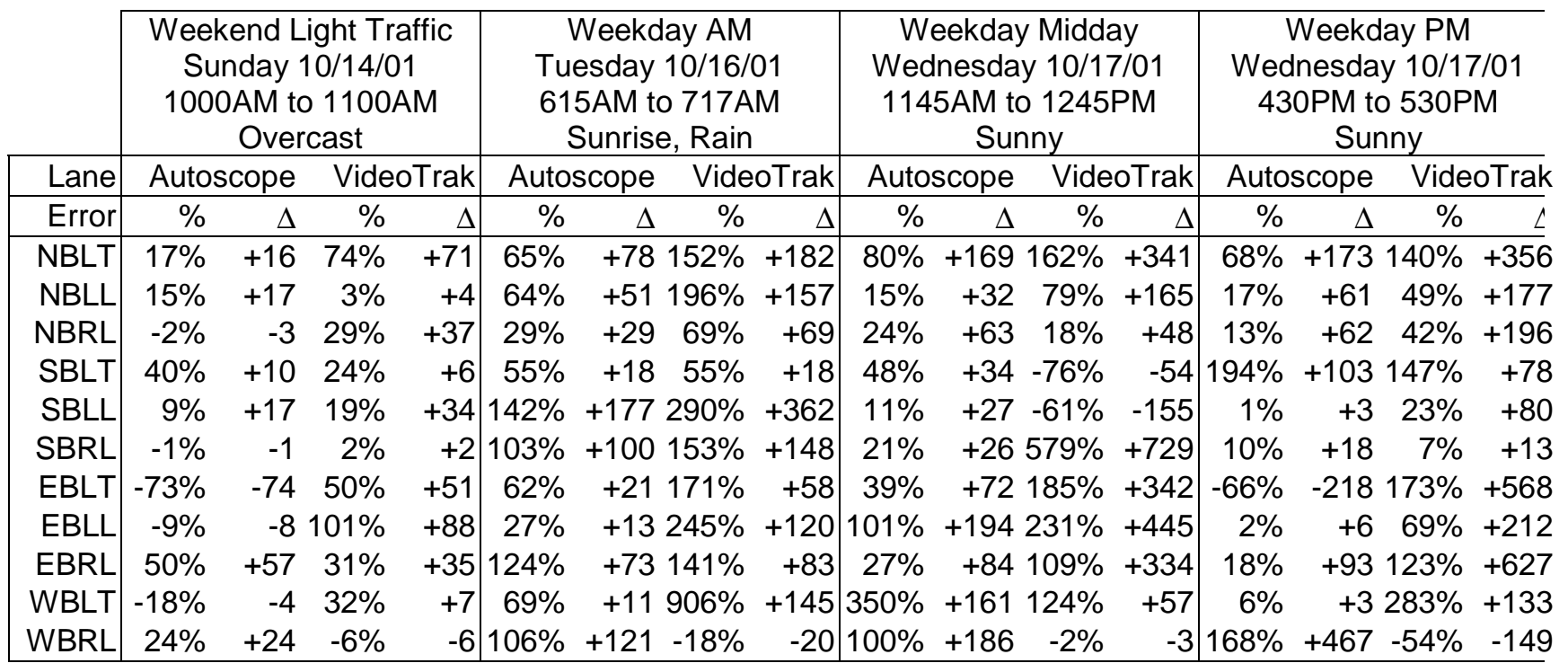




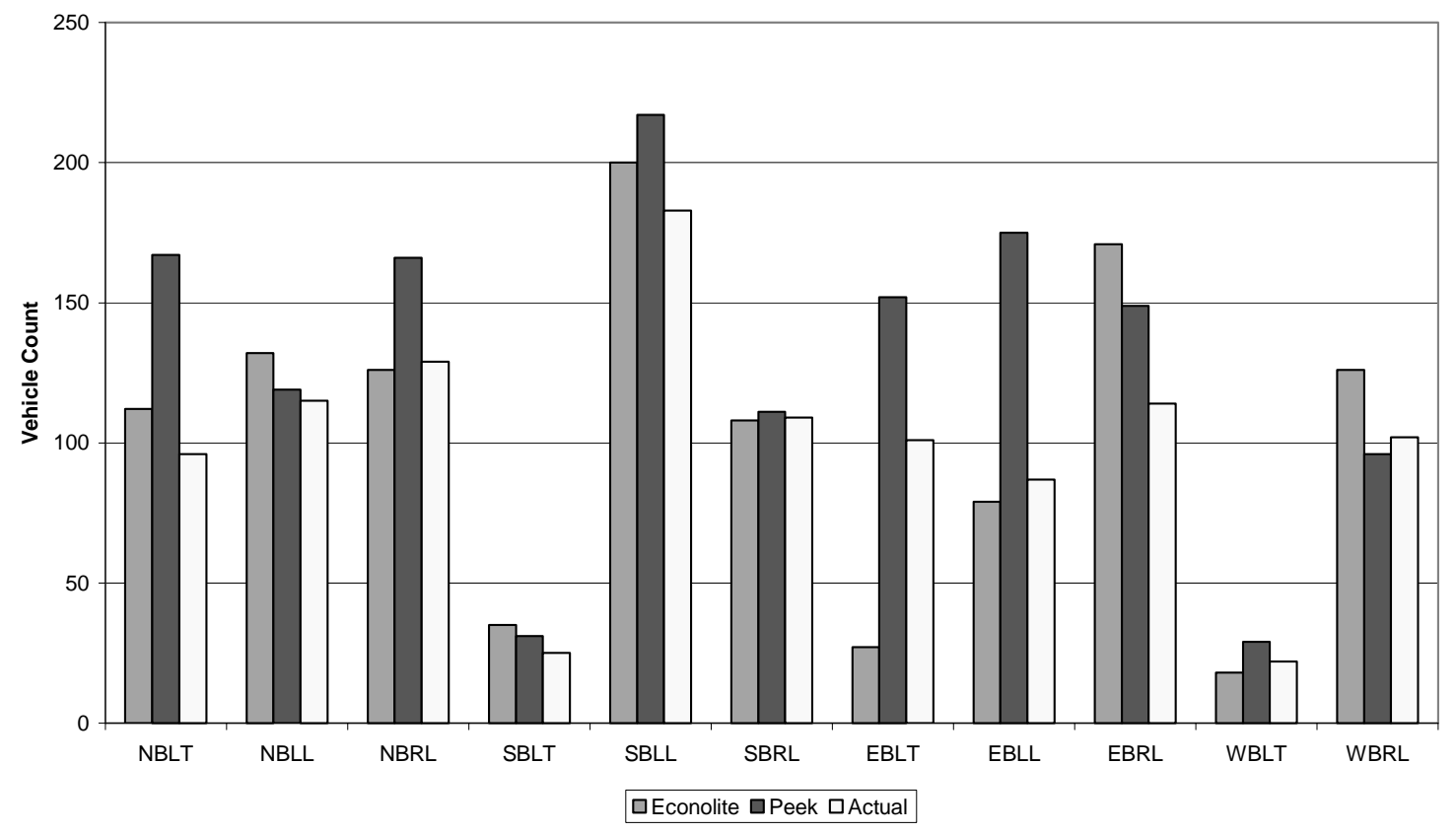

Figure 9: Test 1 Sunday 10/14/01 10:00-11:00AM, Overcast

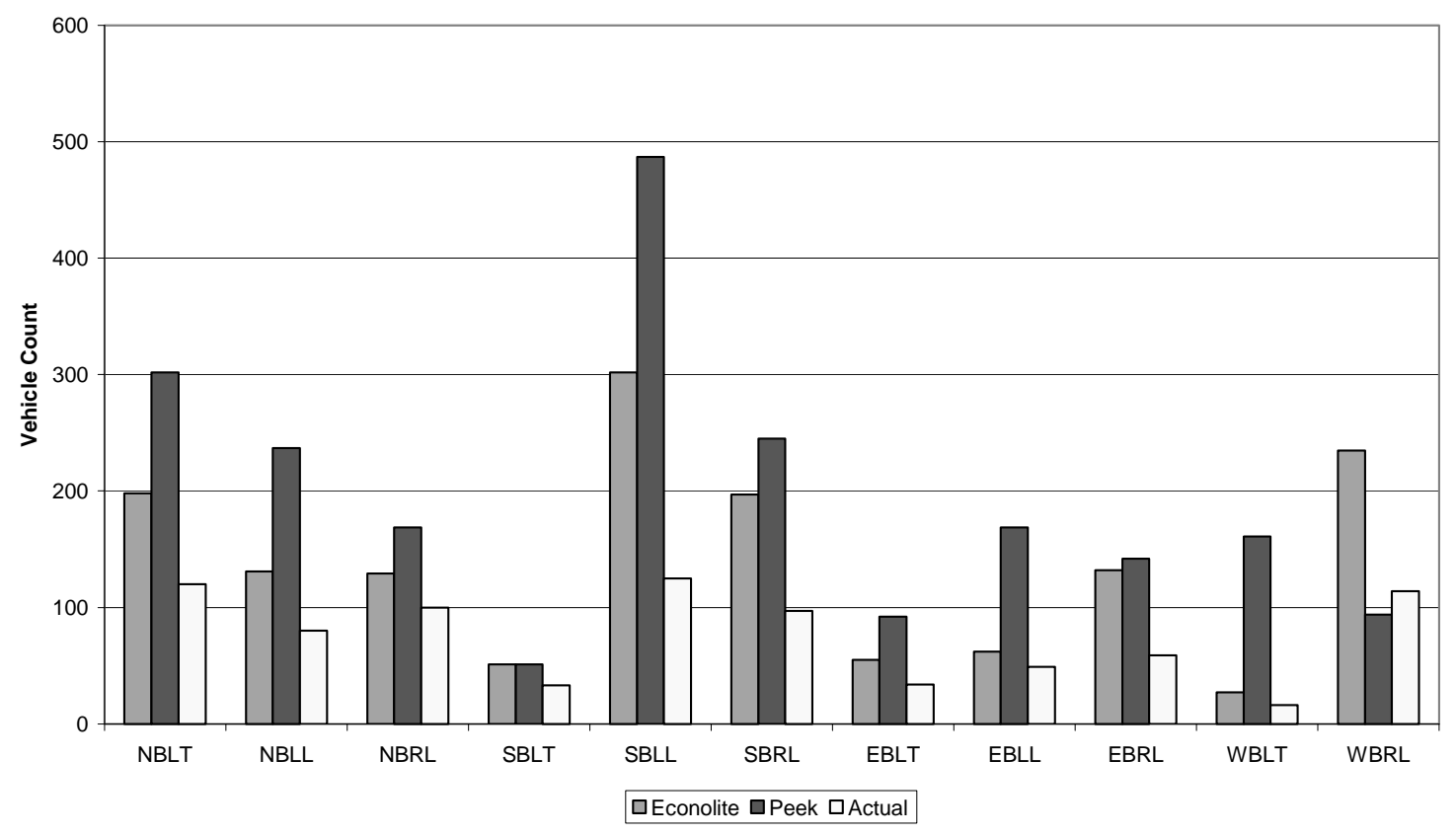

Figure 10: Test 1 Tuesday 10/16/01 6:15-7:15AM, Sunrise, Rain 


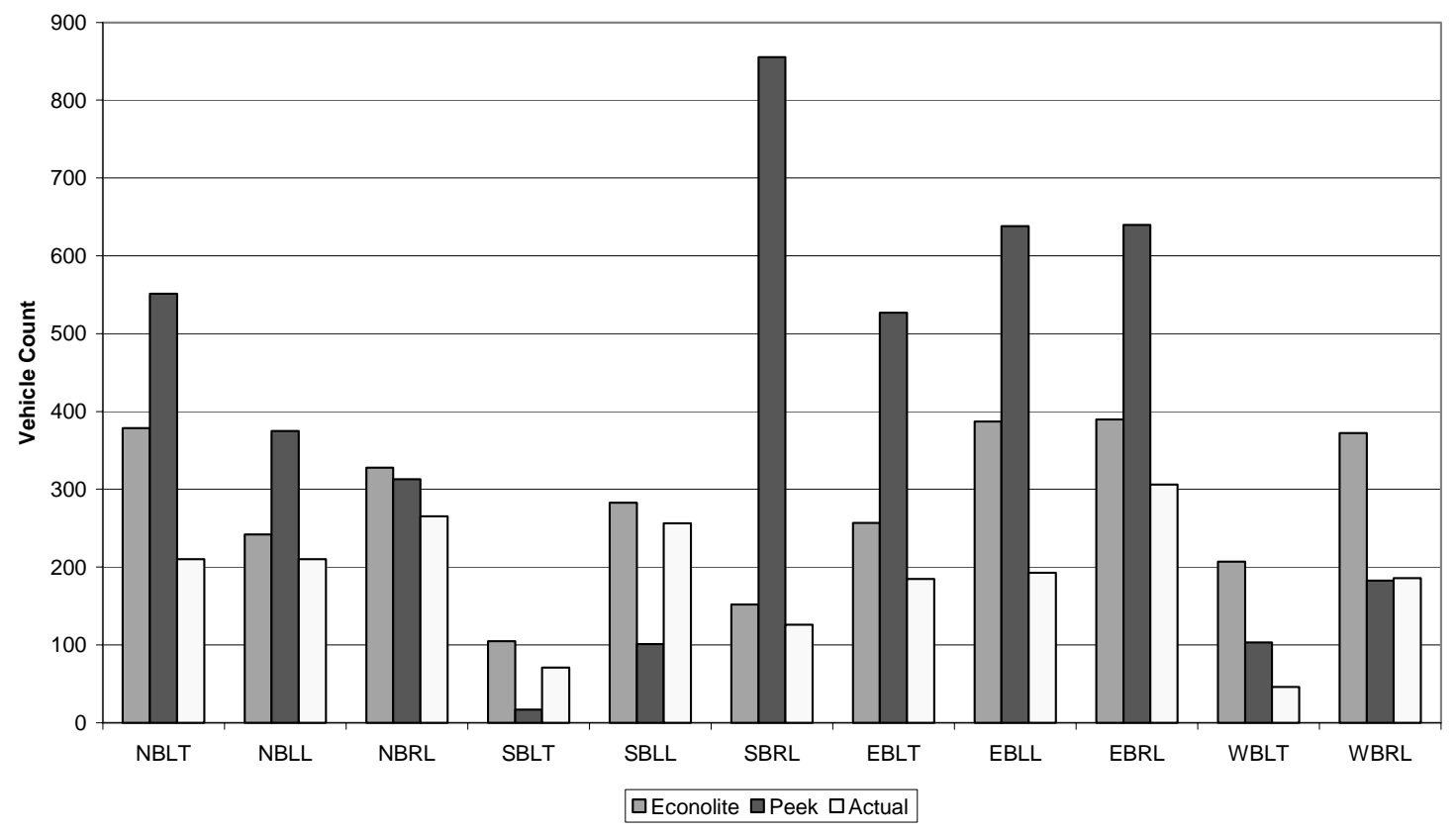

Figure 11: Test 1 Wednesday 10/17/01 11:45AM-12:45PM, Sunny

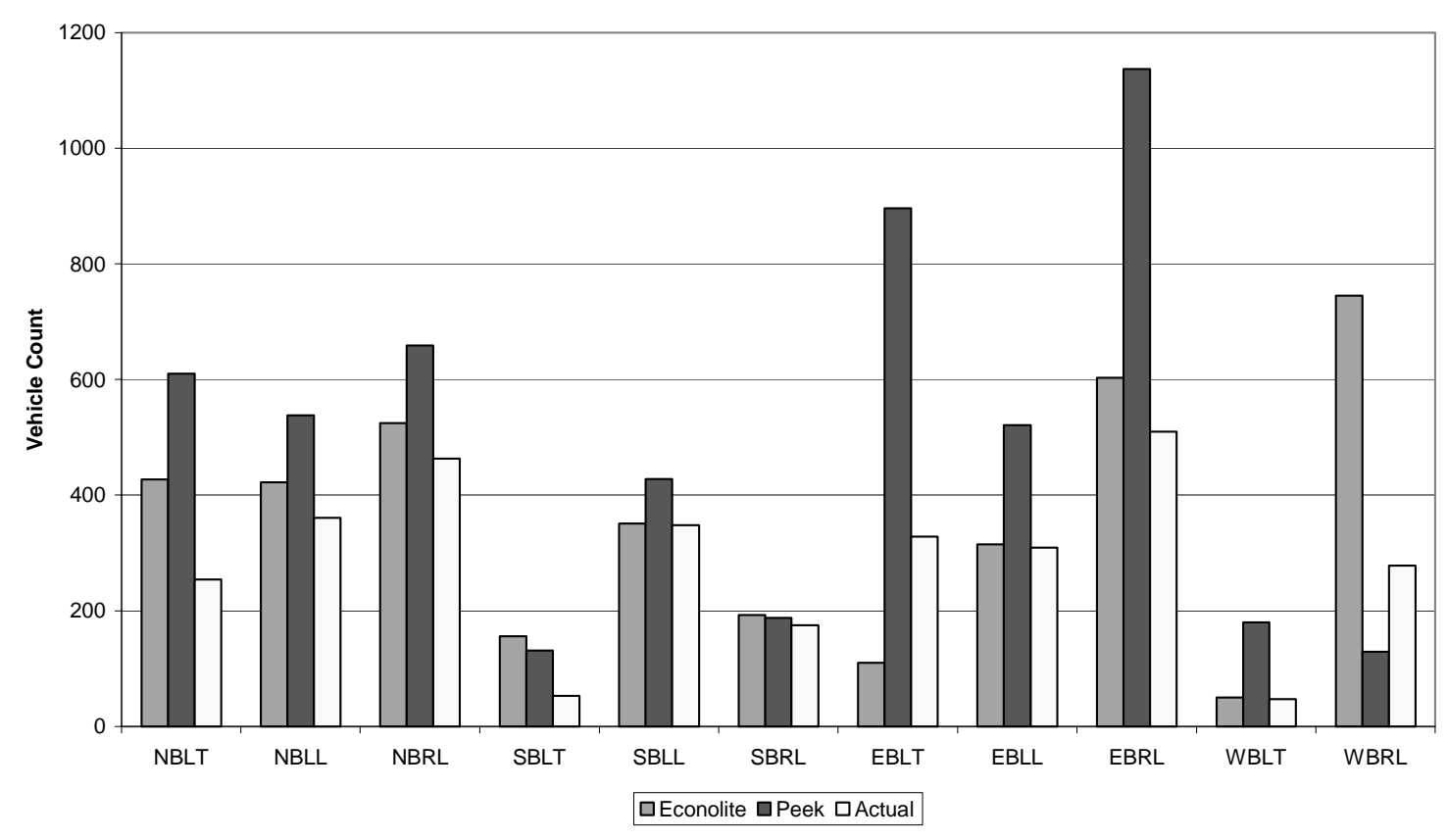

Figure 12: Test 1 Wednesday 10/17/01 4:30-5:30PM, Sunny 


\section{Video Detection Test 2}

Table 18: Test 2 Video Detection Counts

\begin{tabular}{|r|rrr|rrr|}
\cline { 2 - 6 } \multicolumn{1}{c|}{} & $\begin{array}{c}\text { Weekday Midday } \\
\text { Friday 10/26/01 } \\
\text { 1100AM to 100PM } \\
\text { Windy, Cloudy }\end{array}$ & $\begin{array}{c}\text { Weekday Evening } \\
\text { Friday 10/26/01 } \\
\text { 530PM to 630PM } \\
\text { Sunset, Windy }\end{array}$ \\
\hline NBLT & Autoscope VideoTrak & Actual & Autoscope VideoTrak & Actual \\
NBLL & 498 & 1347 & $\mathbf{5 6 4}$ & 355 & 566 & $\mathbf{3 4 3}$ \\
NBRL & 565 & 663 & $\mathbf{4 7 2}$ & 292 & 465 & $\mathbf{2 9 1}$ \\
SBLT & 579 & 711 & $\mathbf{5 7 1}$ & 371 & 519 & $\mathbf{3 6 4}$ \\
SBLL & 566 & 738 & $\mathbf{1 5 5}$ & 220 & 276 & $\mathbf{7 6}$ \\
SBRL & 50 & 338 & $\mathbf{3 2 0}$ & 281 & 530 & $\mathbf{3 7 8}$ \\
EBLT & 556 & 996 & $\mathbf{5 1 4}$ & 333 & 334 & $\mathbf{1 9 0}$ \\
EBLL & 391 & 792 & $\mathbf{4 1 5}$ & 319 & 505 & $\mathbf{3 4 3}$ \\
EBRL & 923 & 1001 & $\mathbf{6 7 1}$ & 537 & 859 & $\mathbf{4 5 2}$ \\
WBLT & 132 & 1795 & $\mathbf{1 1 7}$ & 53 & 658 & $\mathbf{5 0}$ \\
WBRL & 968 & 1252 & $\mathbf{4 8 7}$ & 568 & 212 & $\mathbf{2 6 3}$ \\
\hline
\end{tabular}

Table 19: Test 2 Video Detection Error Summary

\begin{tabular}{|c|c|c|c|c|c|c|c|c|}
\hline & \multicolumn{4}{|c|}{$\begin{array}{l}\text { Weekday Midday } \\
\text { Friday 10/26/01 } \\
\text { 1100AM to 100PM } \\
\text { Windy, Cloudy }\end{array}$} & \multicolumn{4}{|c|}{$\begin{array}{l}\text { Weekday Evening } \\
\text { Friday } 10 / 26 / 01 \\
\text { 530PM to 630PM } \\
\text { Sunset, Windy }\end{array}$} \\
\hline Lane & \multicolumn{2}{|c|}{ Autoscope } & \multicolumn{2}{|c|}{ VideoTrak } & \multicolumn{2}{|c|}{ Autoscope } & \multicolumn{2}{|c|}{ VideoTrak } \\
\hline Error & $\%$ & $\Delta$ & $\%$ & $\Delta$ & $\%$ & $\Delta$ & $\%$ & $\Delta$ \\
\hline NBLT & $-12 \%$ & -66 & $139 \%$ & +783 & $3 \%$ & +12 & $65 \%$ & +223 \\
\hline NBLL & $-1 \%$ & -7 & $40 \%$ & +191 & $0 \%$ & +1 & $60 \%$ & +174 \\
\hline NBRL & $1 \%$ & +8 & $25 \%$ & +140 & $2 \%$ & +7 & $43 \%$ & +155 \\
\hline SBLT & $38 \%$ & +59 & $85 \%$ & +132 & $189 \%$ & +144 & $263 \%$ & +200 \\
\hline SBLL & $-13 \%$ & -88 & $13 \%$ & +84 & $-26 \%$ & -97 & $40 \%$ & +152 \\
\hline SBRL & $-84 \%$ & -270 & $6 \%$ & +18 & $-76 \%$ & -145 & $76 \%$ & +144 \\
\hline EBLT & $8 \%$ & +42 & $94 \%$ & +482 & $-3 \%$ & -10 & $-46 \%$ & -158 \\
\hline EBLL & $-6 \%$ & -24 & $91 \%$ & +377 & $4 \%$ & +12 & $64 \%$ & +198 \\
\hline EBRL & $38 \%$ & +252 & $49 \%$ & +330 & $19 \%$ & +85 & $90 \%$ & +407 \\
\hline WBLT & $13 \%$ & +15 & $1434 \%$ & $+1,678$ & $6 \%$ & +3 & $1216 \%$ & +608 \\
\hline WBRL & $99 \%$ & +481 & $157 \%$ & +765 & $116 \%$ & +305 & $-19 \%$ & -51 \\
\hline
\end{tabular}




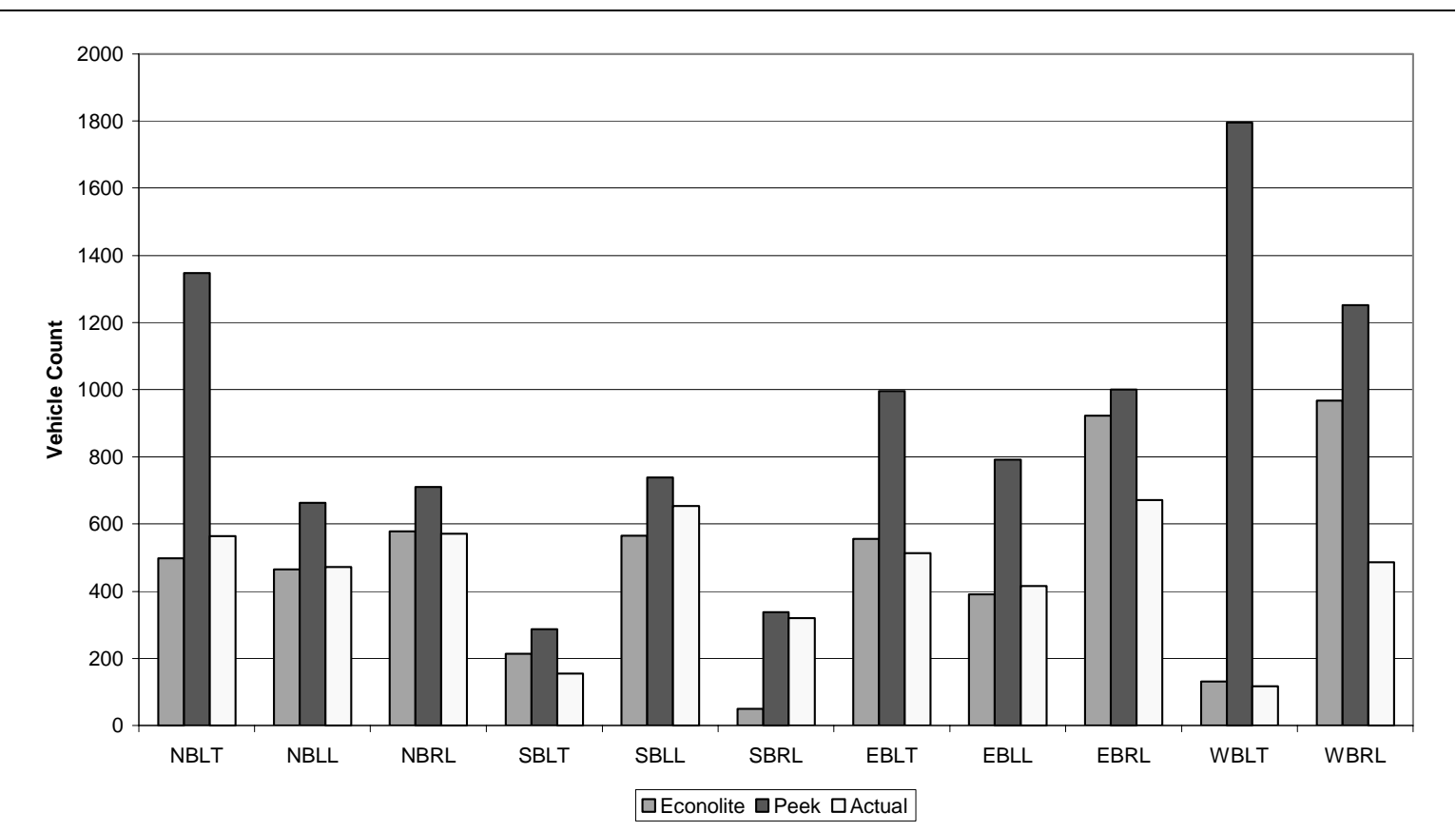

Figure 13: Test 2 Friday 10/26/01 11:00AM-1:00PM, Cloudy, Windy

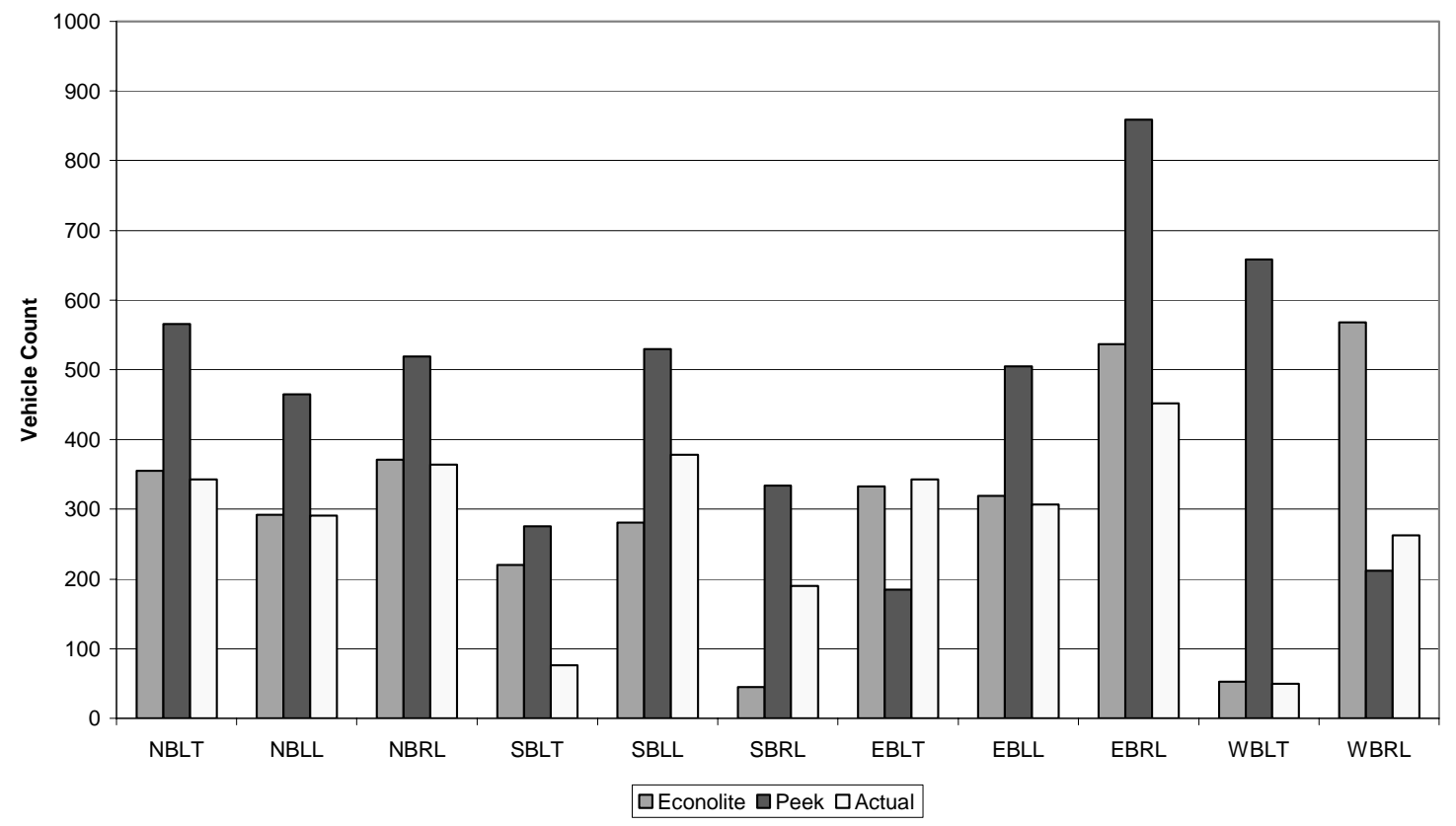

Figure 14: Test 2 Friday 10/26/01 5:30-6:30PM, Sunset, Windy 